\title{
Machine learning applications for Radiomics
}

Citation for published version (APA):

Parmar, C. (2017). Machine learning applications for Radiomics: towards robust non-invasive predictors in clinical oncology. [Doctoral Thesis, Maastricht University]. Datawyse / Universitaire Pers Maastricht. https://doi.org/10.26481/dis.20170518cmp

Document status and date:

Published: 01/01/2017

DOI:

$10.26481 /$ dis. $20170518 \mathrm{cmp}$

Document Version:

Publisher's PDF, also known as Version of record

\section{Please check the document version of this publication:}

- A submitted manuscript is the version of the article upon submission and before peer-review. There can be important differences between the submitted version and the official published version of record.

People interested in the research are advised to contact the author for the final version of the publication, or visit the DOI to the publisher's website.

- The final author version and the galley proof are versions of the publication after peer review.

- The final published version features the final layout of the paper including the volume, issue and page numbers.

Link to publication

\footnotetext{
General rights rights.

- You may freely distribute the URL identifying the publication in the public portal. please follow below link for the End User Agreement:

www.umlib.nl/taverne-license

Take down policy

If you believe that this document breaches copyright please contact us at:

repository@maastrichtuniversity.nl

providing details and we will investigate your claim.
}

Copyright and moral rights for the publications made accessible in the public portal are retained by the authors and/or other copyright owners and it is a condition of accessing publications that users recognise and abide by the legal requirements associated with these

- Users may download and print one copy of any publication from the public portal for the purpose of private study or research.

- You may not further distribute the material or use it for any profit-making activity or commercial gain

If the publication is distributed under the terms of Article $25 \mathrm{fa}$ of the Dutch Copyright Act, indicated by the "Taverne" license above, 

Cover illustration:

Royale Cheese, Ahmedabad 380 006, India

Designer - Niral Desai

C 2017 Royale Cheese, India

Production: Datawyse | Universitaire Pers Maastricht ISBN: 9789461596956

(C) Chintan Parmar, Maastricht 2017 


\title{
Machine learning applications for Radiomics:
}

\author{
Towards robust non-invasive predictors in clinical \\ oncology
}

\author{
DISSERTATION \\ to obtain the doctoral degree \\ at Maastricht University, \\ on the authority of the Rector Magnificus Prof.dr. Rianne M. Letschert, \\ in accordance with the decision of the Board of Deans, \\ to be defended in public on \\ Thursday \\ May $18^{\text {th }}, 2017$ at $12: 00$ hours
}

by

Chintan Parmar 


\section{Promotor}

Prof. dr. Ph. Lambin

\section{Co-promotors}

Dr. Ir. H. J. W. L. Aerts (Harvard University)

\section{Assessment committee}

Prof. dr. R. G. H. Beets-Tan (voorzitter)

Prof. dr. U. van der Heide (Leiden University Medical Center)

Prof. dr. W. J. Niessen (Erasmus University Medical Center)

Prof. dr. S. G. F. Robben

Prof. dr. F. J. W. Verhaegen

The work presented in this thesis is made pssible by the financial support of: CTMM framework (AIRFORCE project, grant 030-103), euroCAT (IVA Interreg - www.eurocat.info), the Dutch Cancer Society (KWF UM 20115020, KWF UM 2009-4454) and the National Institute of Health (NIH-USA U24CA194354, and NIH-USA U01CA190234). 


\section{Contents}

Chapter 1 General introduction and outline of the thesis 7

$\begin{array}{lll}\text { PART } 1 & \text { Assessing variability in radiomic predictors } & 19\end{array}$

Chapter 2 Volumetric CT-based segmentation of NSCLC using 3D-Slicer

Chapter 3 Robust lung nodule segmentation using the 3D Slicer chest imaging platform

Chapter 4 Robust radiomics feature quantification using semiautomatic volumetric segmentation

PART 2 Machine learning methods for Radiomics

Chapter 5 Decoding tumour phenotype by noninvasive imaging using a quantitative radiomics approach

Chapter 6 Radiomic feature clusters and prognostic signatures specific for Lung and Head \& Neck cancer

Chapter 7 Exploratory study to identify radiomics classifiers of Lung cancer histology

Chapter 8 Machine learning methods for quantitative radiomic biomarkers

Chapter 9 Radiomic machine learning classifiers for prognostic biomarkers of Head \& Neck cancer

Chapter 10 General discussion and future perspectives

Summary

Societal impact and valorizations

Acknowledgements

Curriculum vitae

Scientific publications 

Chapter

General introduction and outline of the thesis 



\section{INTRODUCTION}

Cancer is a leading cause of death worldwide. According to global cancer statistics, approximately 14.1 million new cancer cases and 8.2 million cancer deaths occurred worldwide in the year $2012^{1}$. The global burden of cancer is expected to increase due to the growth and aging of world population as well as with the adoption of lifestyle behaviors like smoking, poor diet, physical inactivity and reproductive changes, which are known to increase cancer risks ${ }^{1}$. It is expected that annual cancer cases could rise up to 22 million within the next two decades. A substantial proportion of the worldwide burden of cancer can be prevented through the widespread application of existing cancer control knowledge, early detection, appropriate treatments and palliative care. Therefore, significant efforts and policies are being pushed forward worldwide to facilitate technological advances in fundamental research and clinical care for cancer prognosis and prevention. Due to these efforts, in recent years, many new diagnostic and treatment modalities have become available in clinical oncology ${ }^{2}$, which can generate high dimensional patient specific data. This increase of patient specific information has led the emergence of precision oncology.

\section{Precision Oncology and Radiomics}

'Precision oncology' refers to the customization of cancer care, where practices and/or therapies are being tailored to individual patients ${ }^{3}$. Such customization process can maximize the success of preventive and therapeutic interventions with minimum side effects. Most of the precision oncology related research has centered on the molecular characterization of tumors using genomics based approaches, which require tissue extraction by tumor biopsies. Discovery and translation research carried out over the last 30 years has led to the identification and validation of a number of "cancer drivers"genes that, when mutated or otherwise dysregulated, can drive malignancy in model system ${ }^{4}$. Despite all these successful examples, the clinical realization of precision oncology is still limited due to several factors. Tumors exhibit spatial and temporal heterogeneity, both between and within tumors ${ }^{5}$. Recently, myriad sub-clones having distinct genetic profiles within each tumor, were observed in a series of renal cell carcinomas ${ }^{6}$. It is this intra-tumor heterogeneity and sub-clone diversity that likely causes the phenotypic variation and hence the differences in treatment/drug response and resistance. Furthermore, heterogeneity exists over the lifetime of a cancer, with differing patterns of genetic changes from initiation through to metastasis and relapse after surgery or therapy ${ }^{7}$. In order to assess the spatial and longitudinal intra-tumor heterogeneity, multiple and repeated tumor biopsies would be needed, which is a bottleneck due to the invasiveness of the procedures. Therefore, non-invasive approaches are necessary to profile tumor phenotype.

Medical imaging, a routinely used diagnostic tool in clinical oncology, has unprecedented potential for tumor characterization. Unlike biopsies, medical imaging is non- 
invasive and can provide information regarding the entire tumor. Furthermore, due to the advances of high-resolution image acquisition machines, differentiation of tumor phenotypic characteristics on medical images has become feasible [see Figure-1] ${ }^{8}$.

A
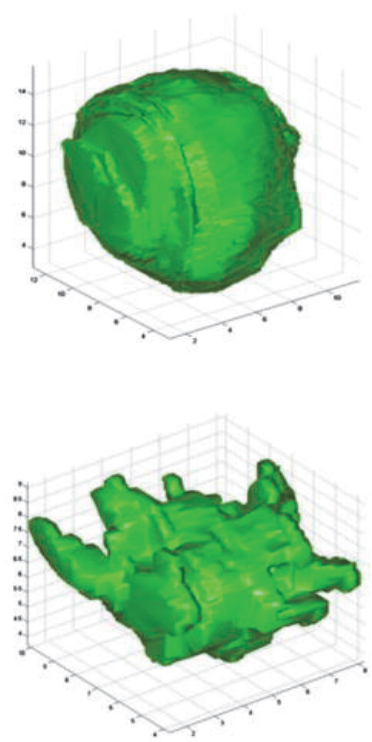

B
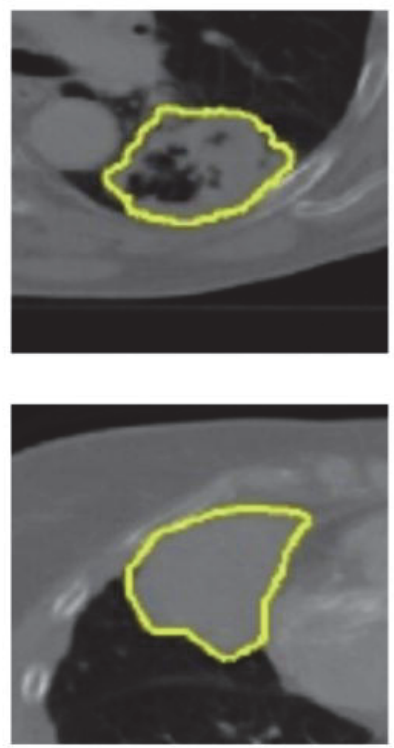

C
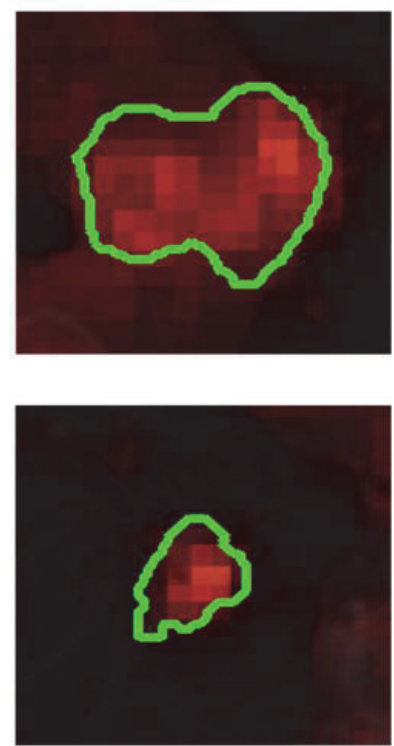

Figure 1. (A) Two representative 3-D representations of a round tumour (top) and spiky tumour (bottom) measured by computed tomography (CT) imaging. (B) Texture differences between non-small cell lung cancer (NSCLC) tumours measured using CT imaging, more heterogeneous (top) and more homogeneous (bottom). (C) Differences of FDG-PET uptake, showing heterogeneous uptake ${ }^{8}$.

However, until recently, medical imaging was still only considered for the detection and qualitative assessment of tumors in clinical oncology. Radiomics, a high throughput, medical image mining approach hypothesizes that with the enriched knowledgebase of image processing and computer vision, different phenotypic characteristics of tumors, including intra/inter tumor heterogeneity, can be quantified in terms of various imaging features ${ }^{8}$. It is expected that these imaging features provide complementary and interchangeable information compared to other sources e.g. demographics, pathology, genomics and that combining these sources of information can enhance precision oncology ${ }^{8}$

\section{The workflow of radiomics}

Figure- $2^{8}$ depicts the processes involved and workflow of the Radiomics. It begins with the image acquisition and standardization. From images, the macroscopic tumor is defined, either with automated/semi-automated segmentation software tools or manually by experienced radiologists or radiation oncologists. Three-dimensional quantita- 
tive imaging features are subsequently extracted from the defined tumor volume. These imaging features involve different shape and size descriptors of tumor and surrounding tissues as well as descriptors of intensity distribution, spatial relationships between various intensity levels, texture heterogeneity patterns in spatial and various transformed domains etc ${ }^{8}$. These extracted image traits are then subjected to a feature selection procedure. After the identification of the most informative and non-redundant features, the selected features are then analyzed for their relationship with treatment outcomes or gene expression. The ultimate goal is to provide accurate risk stratification by incorporating the imaging features into predictive models for treatment outcome and to evaluate their added value to commonly used predictors.

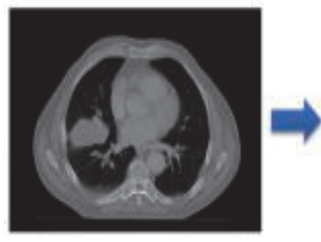

Imaging

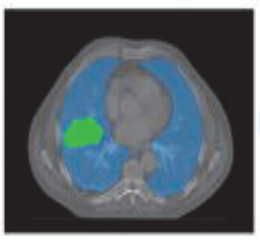

Segmentation

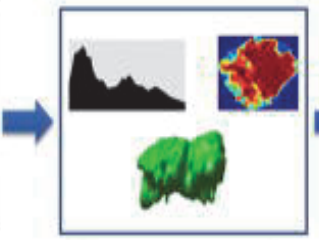

Feature extraction

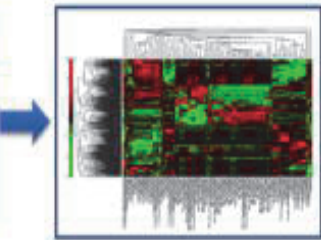

Analysis

Figure 2. The Radiomics workflow. On the medical images, segmentation is performed to define the tumour region. From this region the features are extracted, e.g. features based on tumour intensity, texture and shape. Finally, these features are used for analysis, e.g. the features are assessed for their prognostic power, or linked with stage, or gene expression ${ }^{8}$.

\section{Applications of Radiomics}

Radiomics based applications and research work has significantly increased in recent time, which has been highlighted in several well-written review articles ${ }^{9,10,11,12}$. In order to cover the wide range of applicability, in subsequent section, we briefly mention some selected radiomic applications in clinical oncology.

Diagnosis: Several studies have successfully applied radiomics for enabling the cancer diagnosis. In a study of 147 men with biopsy-proven prostate cancer, Wimber et al ${ }^{13}$ observed that gray level co-occurrence based texture features differentiate malignant and benign prostate tumors on both T2-weighted MR and diffusion-weighted MR images. Moreover, radiomic features extracted from low dose CT images have shown strong discrimination power in stratifying cancerous and malignant NSCLC nodules in national lung screening cohorts ${ }^{14}$.

Tumor prognosis, treatment response and outcomes: Strong prognostic power of radiomic features have been demonstrated using $\mathrm{CT}^{15,16,17,18,19}, \mathrm{MR}^{13,20,21,22}, \mathrm{PET}^{12,23,24}$ and also multi-parametric imaging ${ }^{25,26}$. Kuo et $\mathrm{al}^{27}$ identified association between hepatocellular carcinoma imaging phenotypes and doxorubicin drug response. Also, in a breast cancer study of 58 women, it was found that texture analysis of dynamic contrastenhanced MR images could help in predicting the response to neoadjuvant chemotherapy before its initiation ${ }^{28}$. As far as clinical outcomes are concerned, several studies 
have shown the discriminating capabilities of radiomic features for the stratification of tumor histology ${ }^{29}$, tumor grades or stages ${ }^{30}$, distant metastasis ${ }^{16}$, overall survival and other clinical outcomes ${ }^{31,21,17}$.

Radiomic-Genomic associations: In one of the first radiogenomics study, strong association between contrast enhanced CT based imaging phenotypes and gene expressions was observed in the patients of hepatocellular carcinoma ${ }^{17}$. It was reported that a combination of 28 imaging traits could reconstruct $78 \%$ of the global gene expression profiles, which in turn were linked to cell proliferation, liver synthetic function and patient prognosis ${ }^{17}$. Several other studies have demonstrated the association of radiomic features and gene expression using different cancer types and imaging modalities $20,27,22$

\section{Challenges}

There are few discrete challenges associated with radiomics. Few recent reviews provide detailed overview of these challenges and recommend potential solutions ${ }^{9,10,32,11}$. Following is the brief description of these challenges in lined with the radiomics workflow.

Image acquisition and reconstruction: Most of the published research in literature has been carried out on retrospective imaging data and historically there is lack of harmony in protocols of image acquisitions and reconstructions between different medical institutions. Image acquisition is the primary module of radiomics workflow and therefore lack of standardization could cause unknown effect that could significantly influence radiomic analyses. Hence, standardization of image acquisition and reconstruction protocol without affecting the traditional diagnostic applications of medical imaging is required.

Segmentation: Inter reader variability in tumor delineation is a well-known phenomena in clinical oncology ${ }^{33}$. As majority of radiomic features quantify tumor phenotypes, precise and robust tumor delineation would be needed. Furthermore, manual tumor delineation is relatively time consuming process and that could be a bottleneck for the high-throughput data mining research like radiomics where large patient cohorts are analysed. Therefor, efficient, accurate, robust and automatic (or semiautomatic with minimal manual interaction) segmentation tools are needed. Moreover, the reproducibility of these tools needs to be assessed for radiomics.

Feature extraction and quantification: Once tumors are delineated, different types of imaging features can be extracted e.g. first order intensity histogram based features, second order textural features, shape and size descriptors etc. Moreover, images can be transformed using several image transformation and features can be extracted from the transformed images. Several technical settings need to be implemented before feature extraction. For example, in order to quantify grey level co-occurrence based textural features, parameters like directions, intensity quantization etc. need to be chosen. As there are many categories of features, there are many such inherent parameters. How 
to choose optimal parameter for each feature category? How sensitive these features are with respect to these parameters? All these questions need to be addressed.

Statistical analysis: Statistical analysis, the final component of radiomics workflow, employs various statistical and heuristic approaches. These approaches should be able to identify reproducible findings that could be potentially translated to clinic. Radiomics involves a large number of features. Moreover, for the statistical validity of an analysis, it is recommended to have fewer features than the number of samples involved. Furthermore, not all radiomic features could be important for each and every application. Therefore, dimensionality reduction and selection of application-specific features are necessary for achieving high performance. Similarly, the choice of the prediction modelling method could also be vital in achieving higher performance. Therefore, state of the art machine learning methods for feature selection and prediction/prognostic modelling should be evaluated for radiomic analyses.

\section{Objectives and outline of the thesis}

In order to enhance the applications of radiomics, in this thesis, we tried to address the above mentioned challenges by conducting different radiomic analyses on large patient cohorts of non small cell lung cancer and head and neck cancer. The whole work of this thesis is represented in two parts. First part focuses on challenges related to image acquisitions and standardisation protocols as well as related to segmentations. The second part contains the studies investigating different machine-learning based statistical approaches for radiomic analyses. To begin with, Chapter 1 provides the general introduction of radiomics including the workflow, applications, and associated challenges. Also, it briefly outlines the organization of the thesis.

\section{Part-1 Assessing variability in radiomics:}

The first part focuses on assessing publicly available semi-automatic segmentation tools. These tools were evaluated in terms of their efficiency and reproducibility and were compared to the manual segmentations. Moreover, reproducibility of radiomic features with respect to different segmentation methods were also assessed.

Chapter-2 presents the study where we assessed the clinical relevance of a semiautomatic (CT)-based segmentation method using the competitive region-growing based algorithm, implemented in the fre and publicly available 3D-Slicer software platform. 3D-Slicer based segmentations were also compared with the manual segmentations and it was observed that 3D-Slicer provides a faster and more robust alternative for segmenting NSCLC tumors.

Chapter-3 describes the applications of single seed segmentation algorithm implemented in publicly available Chest Imaging Platform (CIP). In a low dose CT images of 354 nodules, single seed based semiautomatic segmentations were compared to the manual segmentations of four radiologists. It was observed that CIP segmentations 
displayed superior computational efficiency and robustness for majority of nodules as compared to manual delineation.

In Chapter-4, a 3D-Slicer based semiautomatic volumetric segmentation algorithm, was investigated in terms of its robustness for quantitative imaging feature extraction. It was observed that radiomic features extracted from 3D-Slicer segmentations had significantly higher reproducibility compared to the features extracted from the manual segmentations. The results show that 3D-Slicer segmented tumor volumes provide a better alternative to the manual delineation for feature quantification, as they yield more reproducible imaging descriptors.

\section{Part-2 Machine learning methods for radiomics}

In this part, different unsupervised and supervised machine learning methods were investigated for radiomics based prognostic/predictive analyses. These different methods of feature selection/reduction and prediction modeling were compared in terms of their performance and robustness.

Chapter-5 presents a radiomic analysis of 1,019 patients with lung or head-and-neck cancer. Radiomic features were selected based on their reproducibility against testretest imaging and multiple tumor delineation and subsequently were used for prognostic analysis. It was found that radiomic signature have prognostic power in independent data sets of lung and head-and-neck cancer patients. Moreover, the radiogenomic analysis of this study reveals that a prognostic radiomic signature, capturing intratumour heterogeneity, is also associated with underlying gene-expression patterns.

As observed in Chapter-5, radiomics provides a comprehensive quantification of tumor phenotypes by extracting and mining large number of quantitative image features. To reduce the redundancy and compare the prognostic characteristics of radiomic features across cancer types, cancer-specific radiomic feature clusters in four independent Lung and Head \& Neck (H\&N) cancer cohorts (in total 878 patients) were investigated in Chapter-6. Consensus clustering resulted in eleven and thirteen stable radiomic feature clusters for Lung and $\mathrm{H} \& \mathrm{~N}$ cancer, respectively. These clusters were validated in independent external validation cohorts. This analysis indicated both common as well as cancer-specific clustering and clinical associations of radiomic features.

In an exploratory study presented in Chapter-7, the association between radiomic features and the tumor histologic subtypes (adenocarcinoma and squamous cell carcinoma) were investigated in the cohorts of non-small cell lung cancer patients. Furthermore, in order to predict histologic subtypes, we explored machine-learning methods and independently evaluated their prediction performance. Histological subtypes can influence the choice of a treatment/therapy for lung cancer patients. We observed that radiomic features show significant association with the lung tumor histology. Moreover, radiomics-based multivariate classifiers were independently validated for the prediction of histological subtypes. 
Highly accurate and reliable machine-learning approaches can drive the success of radiomic applications in clinical care. In Chapter-8, fourteen feature selection methods and twelve classification methods were examined in terms of their performance and stability for predicting overall survival in two independent lung cancer cohorts. To ensure the unbiased evaluation of different machine-learning methods, publicly available implementations along with reported parameter configurations were used. The variability analysis indicated that the choice of classification method is the most dominant source of performance variation. Identification of optimal machine-learning methods for radiomic applications is a crucial step towards stable and clinically relevant radiomic biomarkers, providing a non-invasive way of quantifying and monitoring tumorphenotypic characteristics in clinical practice.

Chapter-9 presents the radiomic study of head \& neck cancer cohorts where different machine-learning methods were evaluated in terms of their performance and stability for predicting overall survival in two independent cohorts. Feature selection and classification methods were compared using a previously reported unbiased evaluation framework of chapter-8. Investigations related to performance variability echoed the similar conclusion as Chapter-8 that the choice of classification method is the major factor driving the performance variation. This study identified prognostic and reliable machine-learning methods for the prediction of overall survival of head and neck cancer patients.

\section{GENERAL DISCUSSION AND FUTURE PERSPECTIVES}

Chapter 10 provides a general discussion of the results presented in this thesis and related future perspectives. 


\section{REFERENCES}

1. Torre, L. A. et al. Global cancer statistics, 2012. CA Cancer J. Clin. 65, 87-108 (2015).

2. Vogelzang, N. J. et al. Clinical cancer advances 2011: Annual Report on Progress Against Cancer from the American Society of Clinical Oncology. J. Clin. Oncol. 30, 88-109 (2012).

3. Garraway, L. A., Verweij, J. \& Ballman, K. V. Precision oncology: an overview. J. Clin. Oncol. 31, 18031805 (2013).

4. Cohen, R. L. \& Settleman, J. From cancer genomics to precision oncology-tissue's still an issue. Cell (2014).

5. Gerlinger, M. et al. Intratumor heterogeneity and branched evolution revealed by multiregion sequencing. N. Engl. J. Med. 366, 883-892 (2012).

6. Gerlinger, M. et al. Genomic architecture and evolution of clear cell renal cell carcinomas defined by multiregion sequencing. Nat. Genet. 46, 225-233 (2014).

7. Yap, T. A., Gerlinger, M., Futreal, P. A., Pusztai, L. \& Swanton, C. Intratumor heterogeneity: seeing the wood for the trees. Sci. Transl. Med. 4, 127ps10 (2012).

8. Lambin, P. et al. Radiomics: extracting more information from medical images using advanced feature analysis. Eur. J. Cancer 48, 441-446 (2012).

9. Gillies, R. J., Kinahan, P. E. \& Hricak, H. Radiomics: Images Are More than Pictures, They Are Data. Radiology 278, 563-577 (2016).

10. Yip, S. S. F. \& Aerts, H. J. W. L. Applications and limitations of radiomics. Phys. Med. Biol. 61, R150-66 (2016).

11. Aerts, H. J. W. L. The Potential of Radiomic-Based Phenotyping in Precision Medicine: A Review. JAMA Oncol (2016). doi:10.1001/jamaoncol.2016.2631

12. Hatt, M. et al. Characterization of PET/CT images using texture analysis: the past, the present... any future? Eur. J. Nucl. Med. Mol. Imaging 1-15 (2016). doi:10.1007/s00259-016-3427-0

13. Wibmer, A., Hricak, H., Gondo, T. \& Matsumoto, K. Haralick texture analysis of prostate MRI: utility for differentiating non-cancerous prostate from prostate cancer and differentiating prostate cancers with different .... European (2015).

14. Hawkins, S. et al. Predicting Malignant Nodules from Screening CT Scans. J. Thorac. Oncol. (2016). doi:10.1016/j.jtho.2016.07.002

15. Coroller, T. P. et al. Radiomic phenotype features predict pathological response in non-small cell lung cancer. Radiother. Oncol. 119, 480-486 (2016).

16. Coroller, T. P. et al. CT-based radiomic signature predicts distant metastasis in lung adenocarcinoma. Radiother. Oncol. 114, 345-350 (2015).

17. Segal, E. et al. Decoding global gene expression programs in liver cancer by noninvasive imaging. Nat. Biotechnol. 25, 675-680 (2007).

18. Nishino, M. et al. Volumetric Tumor Response and Progression in EGFR-mutant NSCLC Patients Treated with Erlotinib or Gefitinib. Acad. Radiol. 23, 329-336 (2016).

19. Hsu, J.-S. et al. Correlation between EGFR mutation status and computed tomography features in patients with advanced pulmonary adenocarcinoma. J. Thorac. Imaging 29, 357-363 (2014).

20. Itakura, H. et al. Magnetic resonance image features identify glioblastoma phenotypic subtypes with distinct molecular pathway activities. Sci. Transl. Med. 7, 303 ra138 (2015).

21. Nicolasjilwan, M. et al. Addition of MR imaging features and genetic biomarkers strengthens glioblastoma survival prediction in TCGA patients. J. Neuroradiol. 42, 212-221 (2015).

22. Grossmann, P., Gutman, D. A., Dunn, W. D., Jr, Holder, C. A. \& Aerts, H. J. W. L. Imaging-genomics reveals driving pathways of $M R I$ derived volumetric tumor phenotype features in Glioblastoma. BMC Cancer 16, 611 (2016).

23. Chicklore, S. et al. Quantifying tumour heterogeneity in 18F-FDG PET/CT imaging by texture analysis. Eur. J. Nucl. Med. Mol. Imaging 40, 133-140 (2013).

24. Kim, D.-H. et al. Quantification of Intratumoral Metabolic Macroheterogeneity on 18F-FDG PET/CT and Its Prognostic Significance in Pathologic NO Squamous Cell Lung Carcinoma. Clin. Nucl. Med. 41, e70-5 (2016). 
25. Khalvati, F., Wong, A. \& Haider, M. A. Automated prostate cancer detection via comprehensive multiparametric magnetic resonance imaging texture feature models. BMC Med. Imaging 15, 27 (2015).

26. Cui, Y. et al. Prognostic Imaging Biomarkers in Glioblastoma: Development and Independent Validation on the Basis of Multiregion and Quantitative Analysis of MR Images. Radiology 278, 546-553 (2016).

27. Kuo, M. D., Gollub, J., Sirlin, C. B., Ooi, C. \& Chen, X. Radiogenomic analysis to identify imaging phenotypes associated with drug response gene expression programs in hepatocellular carcinoma. J. Vasc. Interv. Radiol. 18, 821-831 (2007).

28. Teruel, J. R., Heldahl, M. G., Goa, P. E. \& Pickles, M. Dynamic contrast-enhanced MRI texture analysis for pretreatment prediction of clinical and pathological response to neoadjuvant chemotherapy in patients with .... NMR Biomed. (2014).

29. Basu, S. et al. Developing a classifier model for lung tumors in CT-scan images. in Systems, Man, and Cybernetics (SMC), 2011 IEEE International Conference on 1306-1312 (ieeexplore.ieee.org, 2011). doi:10.1109/ICSMC.2011.6083840

30. Ganeshan, B., Abaleke, S., Young, R. C. \& Chatwin, C. R. Texture analysis of non-small cell lung cancer on unenhanced computed tomography: initial evidence for a relationship with tumour glucose metabolism and .... Cancer (2010).

31. Jain, R. et al. Outcome prediction in patients with glioblastoma by using imaging, clinical, and genomic biomarkers: focus on the nonenhancing component of the tumor. Radiology 272, 484-493 (2014).

32. Kumar, V. et al. Radiomics: the process and the challenges. Magn. Reson. Imaging 30, 1234-1248 (2012).

33. Velazquez, E. R., Aerts, H., Gu, Y. \& Goldgof, D. B. A semiautomatic CT-based ensemble segmentation of lung tumors: Comparison with oncologists' delineations and with the surgical specimen. Radiother. Oncol. (2012). 



\section{PART}

Assessing variability in radiomic predictors 



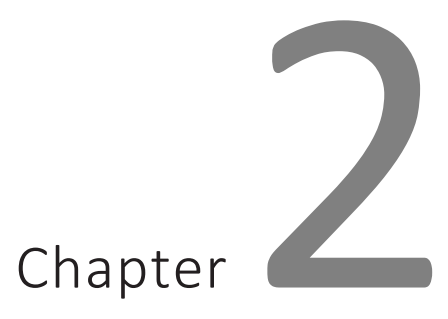

\section{Volumetric CT-based segmentation of NSCLC using 3D-Slicer}

Published in: Nature Scientific Reports. 3, 3529; 2013 Volumetric CT-based segmentation of NSCLC using 3D-Slicer Emmanuel Rios Velazquez*, Chintan Parmar*, Mohammed Jermoumi, Raymond H. Mak, Angela van Baardwijk, Fiona M. Fennessy, John H. Lewis, Dirk De Ruysscher, Ron Kikinis, Philippe Lambin \& Hugo J. W. L. Aerts

*These authors contributed equally to this work 


\section{ABSTRACT}

Accurate volumetric assessment in non-small cell lung cancer (NSCLC) is critical for adequately informing treatments. In this study we assessed the clinical relevance of a semiautomatic computed tomography (CT)-based segmentation method using the competitive region-growing based algorithm, implemented in the free and public available 3DSlicer software platform. We compared the 3D-Slicer segmented volumes by three independent observers, who segmented the primary tumour of 20 NSCLC patients twice, to manual slice-by-slice delineations of five physicians. Furthermore, we compared all tumour contours to the macroscopic diameter of the tumour in pathology, considered as the "gold standard". The 3D-Slicer segmented volumes demonstrated high agreement (overlap fractions>0.90), lower volume variability ( $p=0.0003$ ) and smaller uncertainty areas ( $p=0.0002$ ), compared to manual slice-by-slice delineations.

Furthermore, 3D-Slicer segmentations showed a strong correlation to pathology $(r=$ $0.89,95 \% \mathrm{Cl}, 0.81-0.94)$. Our results show that semiautomatic 3D-Slicer segmentations can be used for accurate contouring and are more stable than manual delineations. Therefore, 3D-Slicer can be employed as a starting point for treatment decisions or for high-throughput data mining research, such as Radiomics, where manual delineating often represent a time-consuming bottleneck. 


\section{INTRODUCTION}

Lung cancer is a disease that affects about 1.6 million individuals worldwide every year ${ }^{1}$. Non-small cell lung cancer (NSCLC) accounts for $85 \%$ of all lung cancer cases and it is characterized by poor prognosis and low survival rates, due to high incidence of locoregional and distant recurrences ${ }^{2}$. In lung cancer, tumour delineation is critical for accurate volumetric assessment to evaluate response to therapy, which can inform treatment decisions. However, tumour delineation can be a source of uncertainty, since typically, the tumour delineation process involves an experienced physician, interpreting and manually contouring computed tomography (CT) alone or combined with Fluorodeoxyglucose (FDG) - positron emission tomography (PET) imaging, on a slice-byslice basis ${ }^{3-6}$. Despite efforts in standardization of CT or FDG-PET-CT image acquisition and standardized guidelines for tumour delineation, definition of lung tumours remains prone to inter-observer variability and is time consuming ${ }^{6-9}$.

To reduce these problems, a number of CT or FDG-PET based semi-automatic methods have been investigated, that aim to provide equivalent segmentations to those delineated manually by physicians, or to provide a starting point for the manual delineation process, thereby reducing the overall required time. The various segmentation methods, that range from simple threshold based methods to complex level set, watershed, or region growing-context based methods, have been compared to manual delineations provided by physicians and compared to the pathological measurements of tumour size, with varying success rates ${ }^{10-16}$. However, the application of these methods is limited, often due to accessibility of the method within the clinical delineation process.

In this study we evaluated the utility of the GrowCut algorithm to segment lung tumours, implemented in 3DSlicer - a free open source software platform for biomedical research ${ }^{17}$. This cellular automaton-based algorithm performs automatic tumour segmentation after drawing boundaries within the image volume. It provides an alternative to the manual slice-by-slice segmentation process and is found to be significantly faster and less user intensive ${ }^{17}$.

Our hypothesis is that 3D-Slicer contours are more stable for inter-observer variation compared to manual contouring. To evaluate the accuracy of the 3D-Slicer segmentations, three independent observers segmented 20 NSCLC patients twice using 3DSlicer.We compared these six 3D-Slicer segmentations to manual delineations provided by five physicians. Furthermore, the segmented volumes were compared with the maximum diameter measured from the tumour after resection, considered as the gold standard. Because 3D-Slicer is publicly available and easily accessible by download, its application in NSCLC could be useful for the clinical investigations where tumour contours are necessary for assessing therapy response, therapy planning, or in highthroughput data mining research of medical imaging in clinical oncology (Radiomics) ${ }^{18-}$ 21 


\section{RESULTS}

Clinical reliability of the 3D slicer's semi-automatic segmentations was measured in terms of its agreement with the CT/PET manual tumour delineations of five independent observers and with pathological measurements after surgery. To quantify the agreement between the manual and 3D-Slicer segmentations, we performed an uncertainty analysis. The uncertainty region was defined as the region that varied between the segmentations of the different observers. In figure 1, the uncertainty region of five manual and six 3D-Slicer segmentations (three observers segmented twice with different seed point initialization) is illustrated. This example shows that the uncertainty region is larger for manual delineations compared to 3D-Slicer.

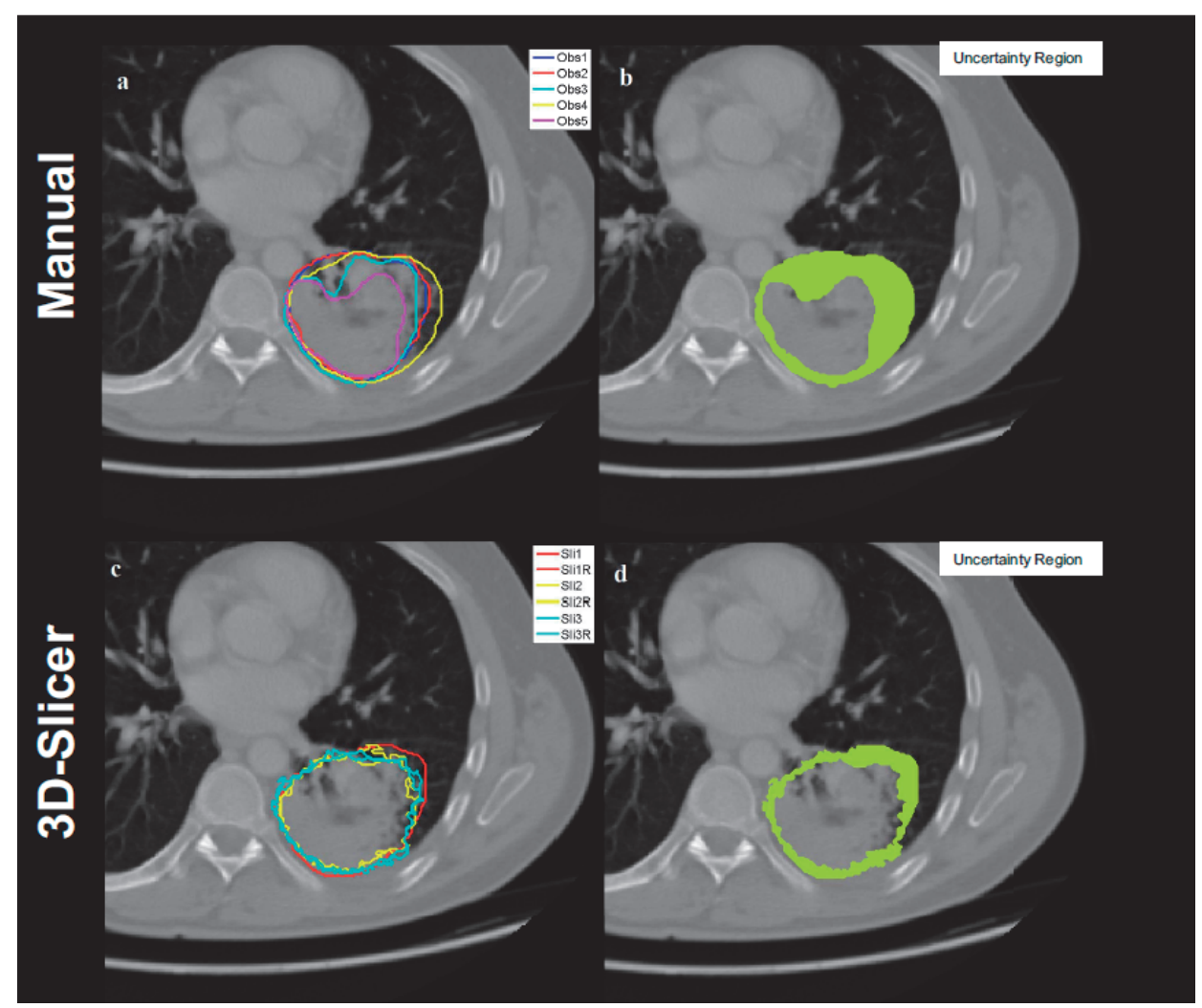

Figure 1. Segmentation uncertainty. Left: representative example showing differences in CT/PET manual delineations (top) and 3D-Slicer segmentations (bottom). Right: This variability is quantified with the uncertainty region, defined as the difference between the observers' agreement and observers' union (highlighted in green). The smaller the uncertainty region is, the lower the variability among multiple contours.

In the Supplementary Figure S1, a heat map depicting the overlap fractions for each patient between the GrowCut segmentations and manual delineations' union and intersection are shown. 
The results demonstrate a high spatial agreement of the manual and 3D-Slicer segmentations.

\section{Overlap fractions}

To examine the spatial agreement of the manual and 3D-Slicer contours, Overlap Fractions (OF) were calculated. OFs were computed between each of the six 3D-Slicer segmentations with the uncertainty region of the manual delineations. The intersection is defined as the inner boundary of the uncertainty region (i.e. the region that all manual observers delineated), and the union as the outer boundary of the uncertainty region (i.e. the region at least one of the manual observers delineated). High OFs were observed with the observers' intersection (mean \pm SD: $94.3 \pm 4.4 \%$, range: $76.8-99.8$ ) and union (mean \pm SD: $97.2 \pm 5.1 \%$; range: 72.6-100) [See figure 2].

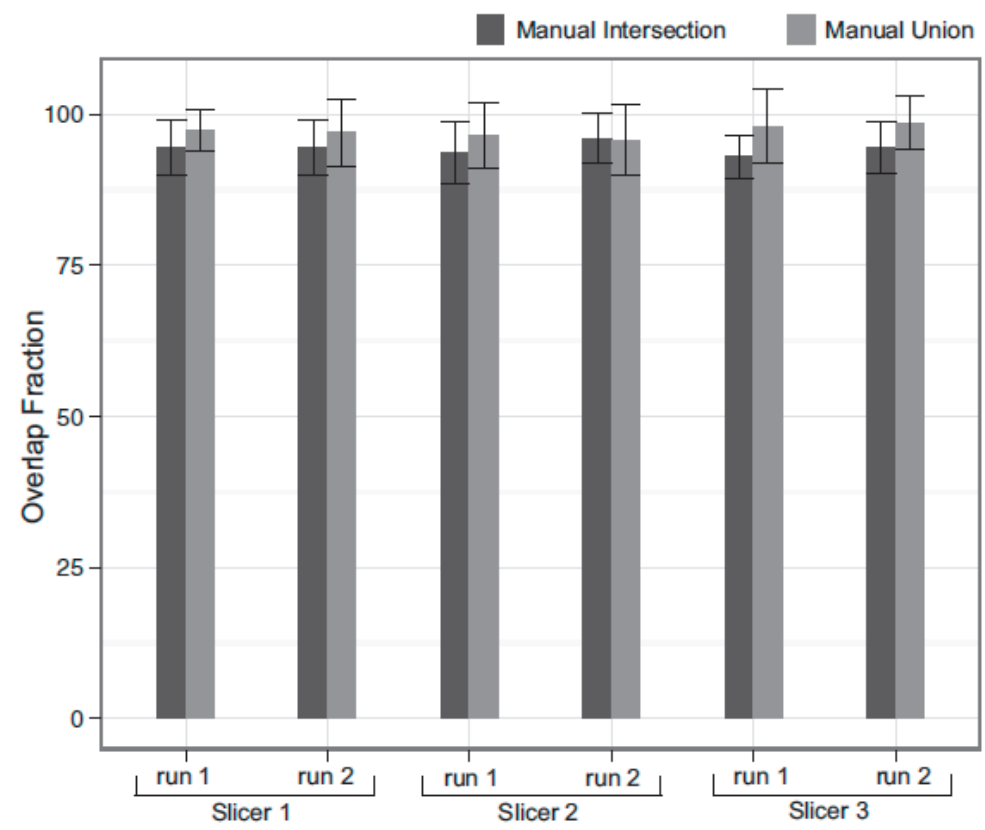

Figure 2. Overlap fractions between the 3D-Slicer segmented volumes and the observers' intersection and union volumes. High overlap fraction indicates high agreement (spatial overlap) between volumes.

\section{Uncertainty regions}

To investigate the robustness of 3D-Slicer segmentations we compared its uncertainty region against the manual uncertainty region [Figure 1]. The analysis showed that the uncertainty region, defined as the difference between uncertainty region inner and outer boundaries, was smaller for the 3D-Slicer segmentations [See Figure 3A]. Manual 
delineations had significantly larger uncertainty areas compared to 3D-Slicer segmentations (Wilcoxon test $p=0.0002$ ).

\section{Segmented volumes}

We then investigated the volumes of the segmentations. There was a high agreement between the volumes of the manual and 3D-Slicer contours, as we found no statistically significant difference between the volumes of the five manual delineations and six 3DSlicer segmentations, using Kruskal-Wallis one-way analysis of variance ( $p=0.98)$. Figure $3 \mathrm{~B}$, displays the tumour volume variability, for both manual and 3D-Slicer for all patients. In 17 cases (85\%), the volume variability was significantly lower for 3DSlicer segmentations $(p=0.0003)$.

\section{D-Slicer segmentation process}

To investigate the stability of 3DSlicer algorithm against user seed-points initialization, we compared the intra-observer variability for each of the 3D-Slicer users. High overlap fractions were observed for the 3D-Slicer users: 95.01\% $\pm 5.33 \%, 94.11 \% \pm 3.95$ and $97.08 \% \pm 2.54 \%$ [mean \pm SD], respectively.

To assess the duration of the 3D-Slicer segmentation process, we recorded the duration of all segmentation phases. The total segmentation times were in average $10.6 \mathrm{~min}$ (range: 4.85-18.25 min), 9.97 (range 6.39-13.83 min) and $9.94 \mathrm{~min}$ (range: 4.38-20.25 $\mathrm{min})$, for the three 3D-Slicer users respectively. In average, the times measured for each 3D-Slicer segmentation phase were: loading (28 seconds), algorithm initialization (2.79 $\mathrm{min})$, running the 3D-Slicer algorithm (32 seconds) and editing final phase (6.52 min).

\section{Pathology}

Further validation was provided by comparing the maximum diameter of the 3D slicer segmentations with that of the surgical specimen. Strong correlations were observed between the maximum diameter of 3D-Slicer volumes and the macroscopic diameter of the surgical tumours (spearman $r$, mean \pm SD $0.89 \pm 0.05$, range: 0.81-0.94).

Similarly, the maximum diameters of the manual CT/PET delineations were highly correlated with the macroscopic diameter (spearman $r$, mean \pm SD $=0.92 \pm 0.02$, range: 0.91-0.95).

Figure 4 displays the scatter plot between macroscopic diameter and the diameters of CT segmentations (manual and 3D slicer). The diameters of surgery had a range of 1.8-9 and average of $4.5 \pm 2.03$ (mean \pm SD). The manual delineations had a range of $1.42-12.53$ and average of $6.09 \pm 2.71$ (mean \pm SD). The semi-automatic delineations were: range 1.41- 12.20 and average of 6.176 2.89. These twelve different diameter vectors were also compared using the Kruskal-Wallis test and no statistically significant difference was observed ( $p=0.97)$. 

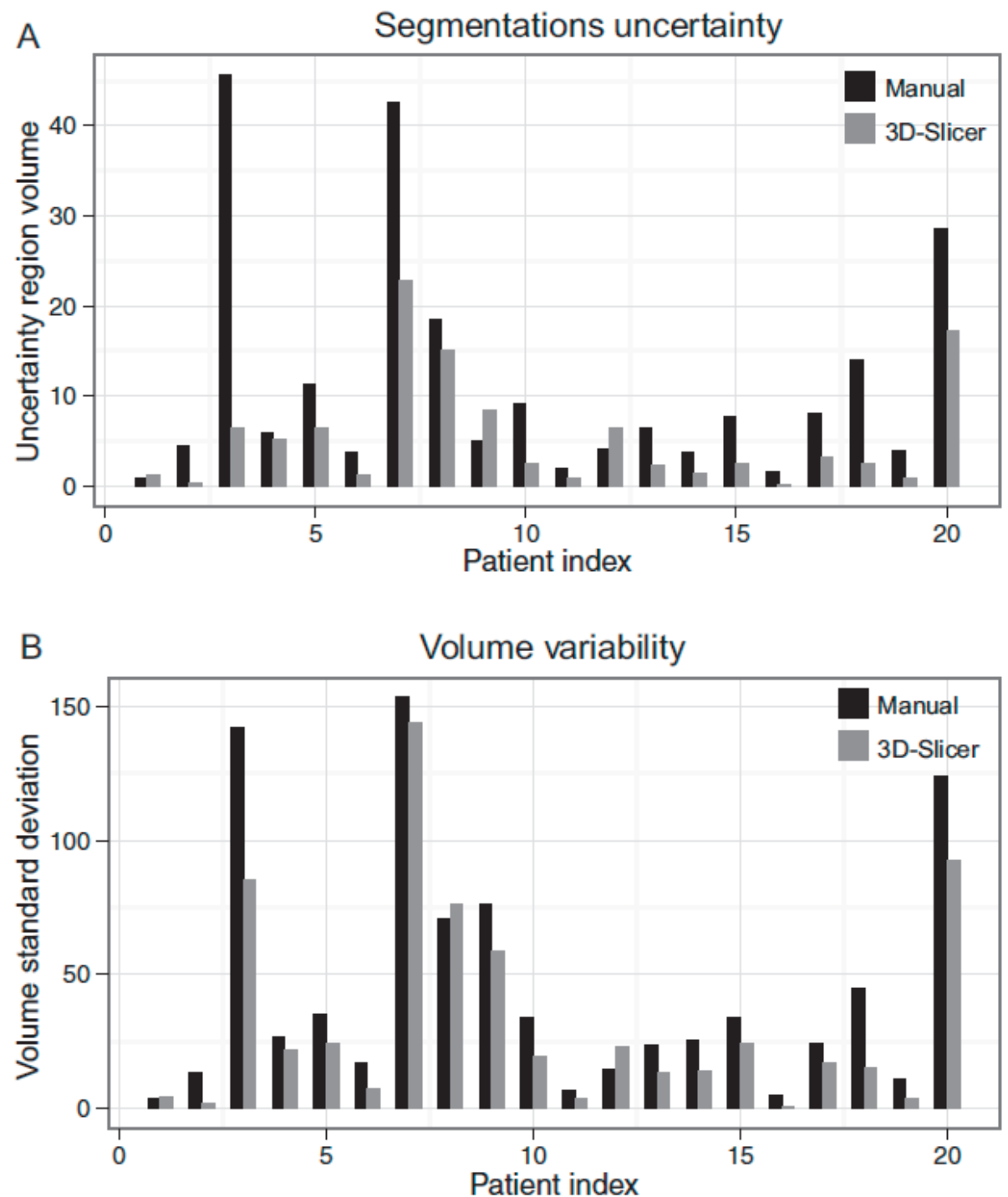

Figure 3.(A): Comparison of volume uncertainty (as defined as the region that varied between the contours of multiple observers) of manual delineations and 3D-Slicer segmentations. See figure 2 for an illustrative example of the uncertainty region. (B): Comparison of volume variability $\left(\mathrm{cm}^{3}\right)$ of observers' manual delineations and 3D-Slicer segmentations. 


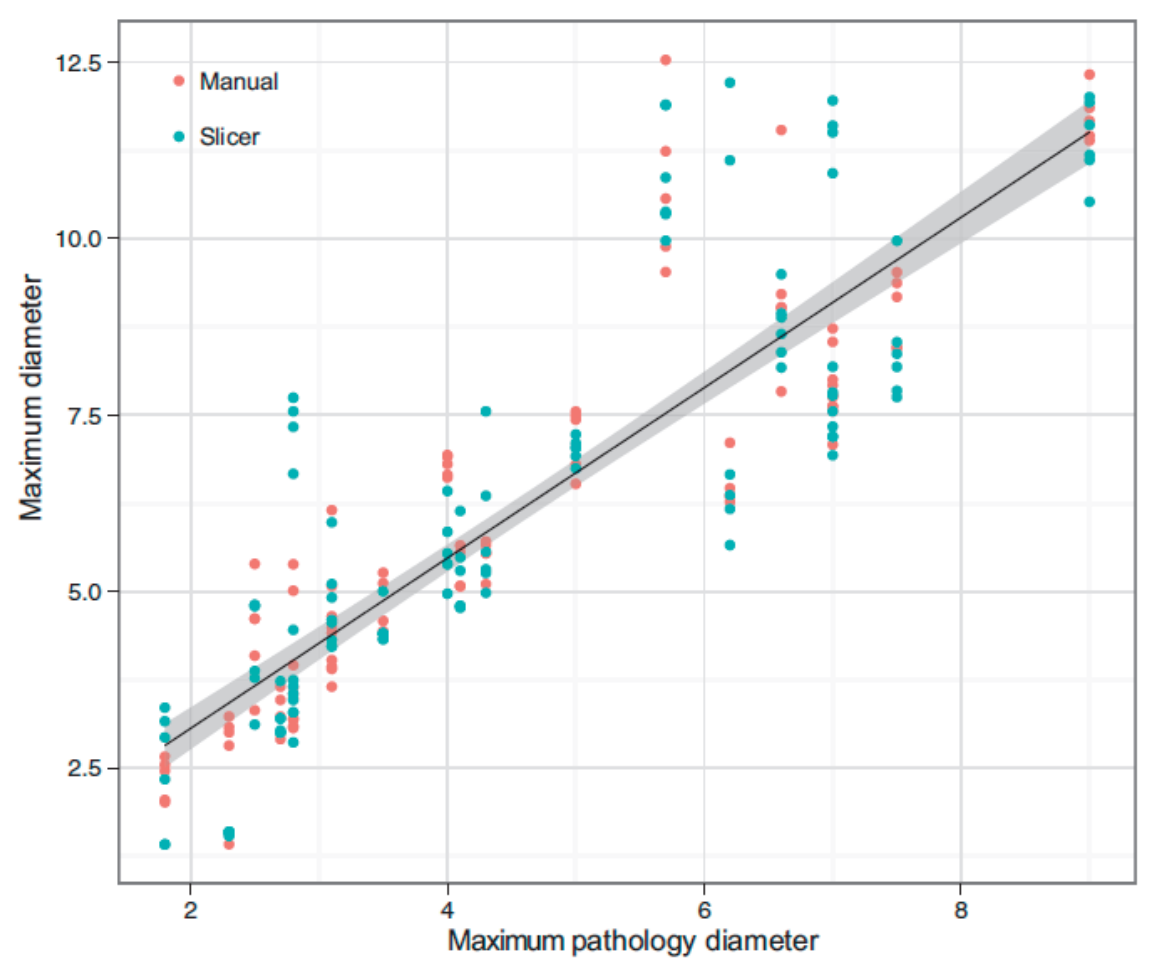

Figure 4. Scatter plot between maximal diameter of surgical specimen and the maximal diameter of computed tomography (CT) segmented volumes for both manual and semiautomatic 3D-Slicer diameters. Spearman's correlation coefficient was $0.89(95 \% \mathrm{Cl}, 0.81-0.94)$.

\section{DISCUSSION}

Despite the efforts in CT-PET imaging standardization and tumour delineation protocols, target definition remains subjected to observer variation. With respect to manual delineations, the addition of PET information to CT imaging in standardized delineation protocols has reduced the observer variability, however, human interaction and interpretation of medical images is still a considerable source of variation ${ }^{3,22,23}$. Furthermore, slice-byslice manual contouring of two-dimensional images is a time consuming process. Here, we evaluated the utility of a freely accessible 3D-Slicer algorithm, a cellular automatonbased algorithm, by performing a volumetric comparison with tumour delineations made by five independent oncologists following standardized protocols ${ }^{24}$, as well as by comparing it with the maximal diameter obtained from pathological measurements.

The volumetric comparison showed that the 3D-Slicer algorithm provides tumour segmentations, statistically equivalent to physicians CT/PET manual contours. To evaluate the accuracy of the 3D-Slicer segmentations, the overlap fraction (\%) was calculated and resulted in high values between the semi-automatically segmented volumes and the intersection (mean \pm SD: $94.3 \pm 4.4 \%$, range: 76.8-99.8) and union (mean \pm SD: $97.2 \pm$ 
5.1\%; range: 72.6-100) of the manual delineations. Importantly, semi-automatic segmentations showed overall lower volume variability $(p=0.0003)$ and smaller uncertainty areas ( $p=0.0002$ ) compared to manual delineations. 3D-Slicer segmentations showed robustness towards user initialization, the OF's between the first Slicer segmentation and the second slicer segmentation were for each user in average: $95.01 \% \pm 5.33 \%$, $94.11 \% \pm 3.95$ and $97.08 \% \pm 2.54 \%$, respectively.

Additionally, we observed a strong correlation between the 3DSlicer segmentations and the maximal diameter as measured on pathological examination $(r=0.89 ; 95 \% \mathrm{Cl}$, 0.81-0.94).

The average time to perform a complete segmentation was 9.8 minutes using Slicer. Loading the images and running the algorithm takes in average half a minute respectively. Due to the retrospective nature of our analysis we were not able to compare the 3D-Slicer segmentation times with the manual delineation times, since those were not available. However 3D slicer's volume segmentation has been shown to be substantially faster and less user intensive compared to manual delineation in other tumour sites ${ }^{17}$. Furthermore, manual delineation is well known to be a very time consuming task.

To minimize observer variability and reduce user interactions, several CT and PET semi-automatic segmentation methods have been introduced. Simple methods such as threshold-based segmentations are widely available but often fail to accurately define the tumour borders ${ }^{10,11,16}$. Various more complex methods have been investigated, including signal-to-background ratio individualized thresholding, watershed-based methods or complex fuzzy locally adaptive thresholding methods ${ }^{11,14,15,25-27}$. These methods have showed generally better correlations with pathology and manual delineations than the simple fixed threshold methods; however they often require significant tuning of algorithm parameters and are not widely available. PET-based methods are intrinsically better choices to segment the highly active metabolic areas of the tumour. In contrast, CT-based methods provide an anatomical segmentation with higher spatial resolution.

In radiation therapy, $\mathrm{CT}$ is the reference imaging modality for treatment planning, and an accurate gross tumour volume definition is fundamental to assure adequate target coverage. Therefore, we believe that CT-based semi-automatic segmentations have clinical utility, if they provide segmentations as accurate as those generated manually by the medical experts, despite the intrinsic CT limitations to distinguish areas of the tumour that are metabolically more active.

Cheebsumon et al, compared several commonly used PET-based segmentation methods with pathology and with a CT manually delineated volume ${ }^{11}$. They reported PET-based methods to have a better agreement with pathology compared to CT delineation. In their study, CT manual delineation significantly overestimated the tumour size compared to pathology. CT manual delineation is known to be prone to inter-observer variation and usually overestimates tumour dimensions. In their exhaustive methods comparison, they lacked a comparison with semi-automatic CT-based segmentation methods, which have shown better correlations with pathology than manual delineations ${ }^{28}$. 
We previously evaluated a CT-based click-and-grow ensemble segmentation (SCES) algorithm, which showed good overlap with medical expert's tumour delineations and with pathological measurements ${ }^{28}$. The SCES also showed robustness towards user initialization, as it involved an iterative segmentation process, with a bootstrapping routine with multiple initializations, which resulted in highly reproducible final segmentations $^{29}$. Unfortunately, this algorithm is only available in commercial packages and therefore not available for the broader community.

A comparison of CT-based and PET-based methods with pathological measurements and manual delineations is still lacking though. We anticipate that methods combining CT and PET information will be the winner in the lung tumour segmentation race, though not all centers are equipped with integrated PET-CT scanners. However, intrinsic differences between CT and PET information should be taken into account. The present 3D-Slicer algorithm, provided accurate tumour segmentations for $85 \%$ of the cases. In three cases the 3D-Slicer failed to define accurately the border, these cases showed larger volume variability with 3D-Slicer compared to manual delineations; two of these cases were large masses with pleural attachment, however only one had a central location. The third case was a very small isolated tumour, adjacent to a main blood vessel, in this case due to the volume size, small variations in border definition due to the adjacent vessel, resulted in significant volume variations. Nevertheless, a medical expert should supervise auto-segmentation algorithms in all cases.

The current correlation between the 3D-Slicer delineation and pathology could possibly be improved if the CT and PET-CT would have been performed in 4D-mode. It is well recognized that a free breathing $\mathrm{CT}$ and even more PET scan will result in blurred edges of the tumour and erroneous CT densities or SUV values. In further research, 4D scans should be used. A general drawback when comparing segmentation algorithms with pathological dimensions is that often only tumour sizes in one dimension are available (maximal diameter). Furthermore, pathological measurements can be affected by tumour shrinkage and deformation after surgery. In this study only the maximal diameter on pathology was compared, which is less prone to error than volumetric comparisons with pathology. The timing-span between the image acquisition and surgery may impact the comparison of the segmentation methods with pathology due to tumour growth. Given the correlation observed with pathological tumour diameter, this time difference may not have a strong impact in the evaluated cases.

In conclusion, the open source 3D-Slicer algorithm, provided tumour segmentations comparable to those manually delineated by physicians and with lower variability. Since the semi-automatic segmentations are statistically comparable to manual delineations and correlated well with pathology, they could be used as a starting point for treatment planning delineations and in high-throughput data mining research, such as Radiomics $^{18-21}$, where manual tumour delineations are often not available, or represent a considerable time consuming bottleneck. 


\section{METHODS}

\section{CT-PET scans}

CT-PET scans. The imaging data was acquired at MAASTRO Clinic in The Netherlands, as reported previously by Baardwijk et $\mathrm{al}^{7}$. In short, twenty consecutive patients with histologically verified non-small cell lung cancer, stage IB-IIIB, were included in this study. All patients received a diagnostic whole body positron emission tomography (PET)computed tomography (CT) scanning (Biograph, SOMATOM Sensation 16 with an ECAT ACCEL PET scanner; Siemens, Erlangen, Germany). Patients were instructed to fast at least six hours before the intravenous administration of ${ }^{18} \mathrm{~F}$-fluoro-2-deoxy-glucose (FDG) (MDS Nordion, Liege, Belgium), followed by physiologic saline (10 mL). The total injected activity of FDG was dependent on the patient weight expressed in kg: (weight* 4) $+20 \mathrm{Mbq}$. Free breathing PET and CT images were acquired after a period of 45 minutes, during which the patient was encouraged to rest. The whole thorax spiral CT scan was acquired with intravenous contrast. The PET images were obtained in 5-min bed positions. The CT data set was used for attenuation correction of PET images. The complete data set was then reconstructed iteratively with a reconstruction increment of $5 \mathrm{~mm}$. Imaging data are available on www.cancerdata.org. This study was conducted according to national laws and guidelines and approved by the appropriate local trial committee at Maastricht University Medical Center (MUMC1), Maastricht, The Netherlands. For more details see Baardwijk et $\mathrm{al}^{7}$.

\section{GrowCut semi-automatic segmentation method in 3D-Slicer}

GrowCut semi-automatic segmentation method in 3D-Slicer. GrowCut is an interactive region growing segmentation method. Given an initial small set of label points the algorithm automatically segments the remaining image by using cellular automation. The algorithm uses a competitive region growing approach and is considered as having good accuracy and speed for the 2D and 3D image segmentation. For $\mathrm{N}$-class segmentation the algorithm needs $\mathrm{N}$ initial sets of pixels (one set corresponding to each class) from user. Using these pixel sets, the algorithm automatically generates the region of interest (ROI), which is the convex hull of the user-labelled pixels with an additional margin. In the next step, it iteratively labels all the pixels in the ROI using the user-given pixel labels. The algorithm converges when all the pixels in the ROI have unchanged labels across several iterations. Pixel labelling is done using a weighted similarity score, which is a function of the neighbouring pixel weights. An unlabelled pixel is labelled corresponding to the neighbouring pixels that have the highest weights. 


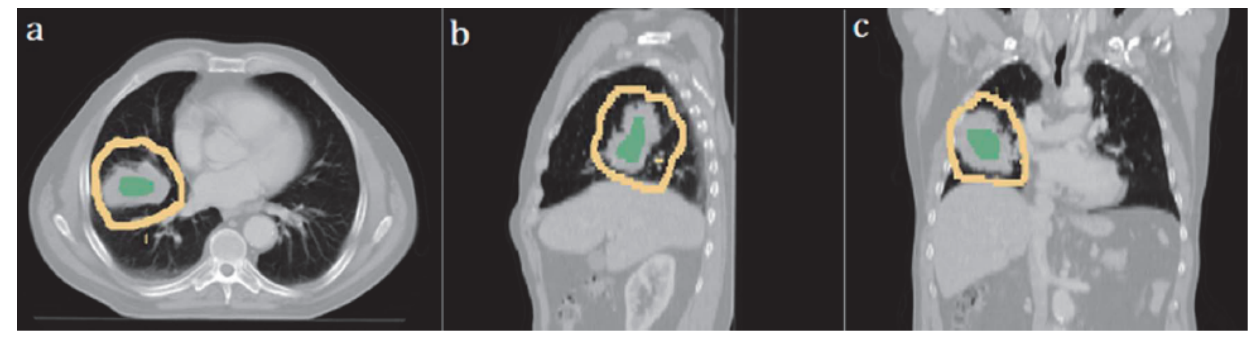

Figure 5. Initialization step of 3D-Slicer segmentation. Marked foreground (green) and background (yellow) are shown. Axial (a), sagittal (b) and coronal (c) views are shown.

\section{NSCLC tumor GrowCut segmentation in 3D-Slicer}

3D-Slicer gives a user friendly GUI as the frontend and an efficient algorithm as the back end for the GrowCut segmentation. After loading the patient data, the process began with the initialization of the foreground and background by marking the area inside and outside the tumour region with few initial seed pixels [Figure 5]. The next step was automatic competing region-growing, which segmented the region of interest into foreground and background. Background and surrounding isolated foreground pixels were removed after visual inspection.

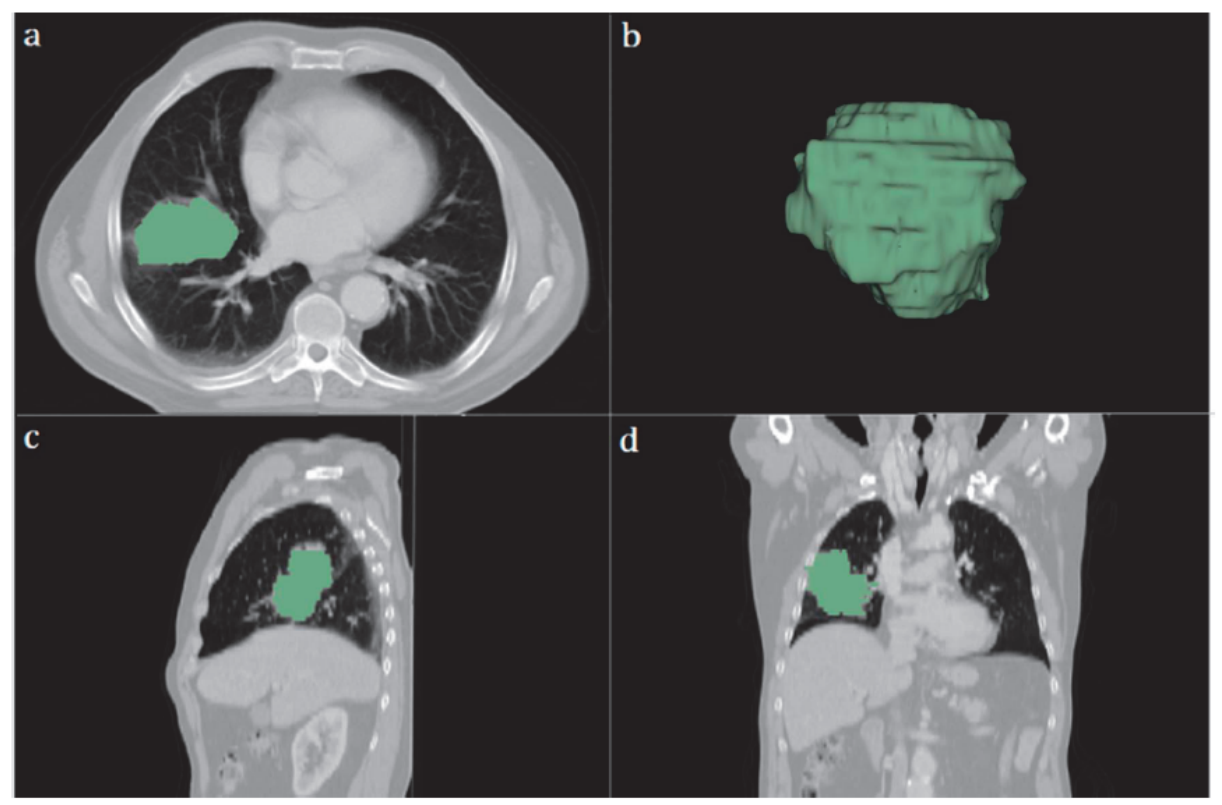

Figure 6. Semi-automatically segmented tumour (green) using 3D-Slicer. Axial (a), three dimensional (b), sagittal (c) and coronal (d) views are shown.

Figure 6 displays the final segmented tumour region. In Supplementary Figure S2 four representative tumour segmentations generated using the 3D-Slicer algorithm are 
compared with the manual delineations of five independent observers. Visual comparison shows a high agreement of the manual delineations with the semiautomatic one. We performed Slicer GrowCut segmentations by three independent users, which repeated the process two times, with a three day interval between each time. Segmentation times using GrowCut were recorded for every step of the analysis.

\section{Manual tumor delineations}

To validate the semiautomatic segmentation method, five radiation oncologist have manually delineated the gross tumour volume (GTV) of the primary tumour, based on fused PET-CT images using standard delineation protocol, which includes fixed windowlevel settings of both CT (lung W 1,700; L 2300, mediastinum W600; L 40) and PET scan (W 30,000; L 15,000 $)^{2,7,24}$. Radiation oncologists were mutually blind of each other's delineations. The primary GTV was defined for each patient based on combined CT and PET information in the axial plane. The radiation oncologists were given transversal, coronal, sagittal and 3D views simultaneously. A treatment planning system (XiO; Computer Medical System, Inc., St. Louis, MO), was used for performing delineations.

\section{Pathology}

The examination of surgical specimen was carried out according to national guidelines ${ }^{7}$. Surgical resections were performed on all the patients. Before slicing, the maximal diameter of the primary tumour was measured by macroscopic examination. The interval time between the CT scan and the surgery or biopsy was in average 39 days (range: 7-112).

\section{Statistical analysis}

Overlap Fraction (OF) was used to evaluate the 3D slicer's segmentations in terms of its spatial overlap with manual delineations. Intersection and union volumes were defined for manual delineations (Figure 1). OFs were calculated between the semiautomatic segmentations and these intersection and union delineations. OF was defined as the as the volume of overlap divided by the smallest volume ${ }^{30}$ :

$$
\text { OFInter }=\frac{S V \cap O B i}{\min \{S V, O B i\}} * 100 \text { and OFunion }=\frac{S V \cap O B u}{\min \{S V, O B u\}} * 100
$$

$S V, O B i$ and $O B u$ are the semiautomatic, observers' intersection and union volumes respectively. OF value of 100 suggests a perfect match while OF value 0 points to two disjoint volumes and thus no match. OF inter indicates whether the semiautomaticsegmentation method covers the common agreement (intersection volume) of the manual delineations while $\mathrm{OF}_{\text {union }}$ indicates whether the algorithm falls within the interobserver variability (union volume). Furthermore, using the above described concept of union and intersection volumes, we calculated and compared the uncertainty of the 
GrowCut segmentations and the manual delineations. The uncertainty was defined as the difference between the union and intersection volumes, which is the area that belongs to the union but not to the intersection volumes. This region can be seen in Figure 1 , highlighted in green. The lower the difference between union and intersection volumes the lower the uncertainty. If all contours were equal, with no variation, the union and intersection volumes would be identical with no uncertainty areas. Overlap fractions were used to compare the first 3D-Slicer segmentation against the second 3DSlicer segmentation for the same observer.

A volume $\left(\mathrm{cm}^{3}\right)$ comparison was also carried out. Volumes calculated from different segmentation methods were compared using the Kruskal-Wallis test. Two methods were considered to be significantly different when the $p$-value was lower than 0.05 .

We compared the volume variability of the 3D-Slicer segmentations against manual delineations using the standard deviation of the 3D-Slicer and manual volumes. The Wilcoxon test was used to compare the volume variability and uncertainty differences between the two types of segmentations.

Spearman correlation coefficient was used to compare the maximal diameter of pathology with the maximal diameter of 3D-Slicer and the manual segmentations.

Further we also compared all these twelve maximal diameter groups: 3D-Slicer (three observers twice), pathology, and five manual using the Kruskal-Wallis one-way analysis of variance. Again groups were considered significantly different when the $p$ value was lower than 0.05. All data are expressed as mean6SD. All the analyses were performed in Matlab (The MathWorks Inc., Natick, MA, USA) and R (R Foundation for Statistical Computing, Vienna, Austria).

\section{ACKNOWLEDGMENTS}

Authors acknowledge financial support from the National Institute of Health (NIH-USA U01 CA 143062-01, Radiomics of NSCLC), the CTMM framework (AIRFORCE project, grant 030-103), EU 6th and 7th framework program (METOXIA, EURECA, ARTFORCE), euroCAT (IVA Interreg - www.eurocat.info), Kankeronderzoekfonds Limburg from the Health Foundation Limburg and the Dutch Cancer Society (KWF UM 2011-5020, KWF UM 2009-4454). Authors also acknowledge financial support from the QulC-ConCePT project (Grant Agreement No. 115151).

\section{SUPPLEMENTARY INFORMATION}

http://www.nature.com/articleassets/npg/srep/2013/131218/srep03529/extref/srep03529-s1.doc 


\section{REFERENCES}

1. Jemal, A. et al. Global cancer statistics. CA Cancer J Clin 61, 69-90 (2011).

2. van Baardwijk, A. et al. Mature results of an individualized radiation dose prescription study based on normal tissue constraints in stages I to III non-smallcell lung cancer. J Clin Oncol 28, 1380-1386 (2010).

3. Steenbakkers, R. J. et al. Observer variation in target volume delineation of lung cancer related to radiation oncologist-computer interaction: a 'Big Brother' evaluation. Radiother Oncol 77, 182-190 (2005).

4. Van de Steene, J. et al. Definition of gross tumor volume in lung cancer: interobserver variability. Radiother Oncol 62, 37-49 (2002).

5. Bowden, P. et al. Measurement of lung tumor volumes using three-dimensional computer planning software. Int J Radiat Oncol Biol Phys 53, 566-573 (2002).

6. Caldwell, C. B. et al. Observer variation in contouring gross tumor volume in patients with poorly defined non-small-cell lung tumors on CT: the impact of 18FDG-hybrid PET fusion. Int J Radiat Oncol Biol Phys 51, 923-931 (2001).

7. van Baardwijk, A. et al. PET-CT-based auto-contouring in non-small-cell lung cancer correlates with pathology and reduces interobserver variability in the delineation of the primary tumor and involved nodal volumes. Int J Radiat Oncol Biol Phys 68, 771-778 (2007).

8. Steenbakkers, R. J. et al. Reduction of observer variation using matched CT-PET for lung cancer delineation: a three-dimensional analysis. Int J Radiat Oncol Biol Phys 64, 435-448 (2006).

9. De Ruysscher, D. PET-CT in radiotherapy for lung cancer. Methods Mol Biol 727, 53-58 (2011).

10. Nestle, U. et al. Comparison of different methods for delineation of 18F-FDG PET-positive tissue for target volume definition in radiotherapy of patients with non-Small cell lung cancer. J Nucl Med 46, 1342-1348 (2005).

11. Cheebsumon, P. et al. Assessment of tumour size in PET/CT lung cancer studies: PET- and CT-based methods compared to pathology. EJNMMI Res 2, 56 (2012).

12. Daisne, J. F. et al. Tri-dimensional automatic segmentation of PET volumes based on measured sourceto-background ratios: influence of reconstruction algorithms. Radiother Oncol 69, 247-250 (2003).

13. Dehmeshki, J., Amin, H., Valdivieso, M. \& Ye, X. Segmentation of pulmonary nodules in thoracic CT scans: a region growing approach. IEEE Trans Med Imaging 27, 467-480 (2008).

14. Schaefer, A. et al. PET-based delineation of tumour volumes in lung cancer: comparison with pathological findings. Eur J Nucl Med Mol Imaging 40, 1233-1244 (2013).

15. Hatt, M. et al. Accurate automatic delineation of heterogeneous functional volumes in positron emission tomography for oncology applications. Int J Radiat Oncol Biol Phys 77, 301-308 (2010).

16. Wu, K. et al. PET CT thresholds for radiotherapy target definition in non-smallcell lung cancer: how close are we to the pathologic findings? Int J Radiat Oncol Biol Phys 77, 699-706 (2009).

17. Egger, J. et al. GBM volumetry using the 3D Slicer medical image computing platform. Sci Rep 3, 1-7 (2013).

18. Kumar, V. et al. Radiomics: the process and the challenges. Magn Reson Imaging 30, 1234-1248 (2012).

19. Lambin, P. et al. Radiomics: extracting more information from medical images using advanced feature analysis. Eur J Cancer 48, 441-446 (2012).

20. Buckler, A. J., Bresolin, L., Dunnick, N. R. \& Sullivan, D. C. A collaborative enterprise for multi-stakeholder participation in the advancement of quantitative imaging. Radiology 258, 906-914 (2011).

21. Buckler, A. J. et al. Quantitative imaging test approval and biomarker qualification: interrelated but distinct activities. Radiology 259, 875-884 (2011).

22. Greco, C., Rosenzweig, K., Cascini, G. L. \& Tamburrini, O. Current status of PET/ CT for tumour volume definition in radiotherapy treatment planning for nonsmall cell lung cancer (NSCLC). Lung Cancer 57, 125-134 (2007).

23. Sonke, J. J. \& Belderbos, J. Adaptive radiotherapy for lung cancer. Semin Radiat Oncol 20, 94-106 (2010).

24. van Baardwijk, A. et al. Individualized radical radiotherapy of non-small-cell lung cancer based on normal tissue dose constraints: a feasibility study. Int J Radiat Oncol Biol Phys 71, 1394-1401 (2008).

25. Ye, X., Beddoe, G. \& Slabaugh, G. Automatic Graph Cut Segmentation of Lesions in CT Using Mean Shift Superpixels. Int J Biomed Imaging 2010, 1-14 (2010). 
26. Daisne, J.-F. o. et al. Tri-dimensional automatic segmentation of PET volumes based on measured source-to-background ratios: influence of reconstruction algorithms. Radiotherapy and Oncology 69, 247-250 (2003).

27. Wanet, M. et al. Gradient-based delineation of the primary GTV on FDG-PET in non-small cell lung cancer: a comparison with threshold-based approaches, CT and surgical specimens. Radiother Oncol 98, 117-125 (2010).

28. Rios Velazquez, E. et al. A semiautomatic CT-based ensemble segmentation of lung tumors: comparison with oncologists' delineations and with the surgical specimen. Radiother Oncol 105, 167-173 (2012).

29. Gu, Y. et al. Automated Delineation of Lung Tumors from CT Images Using a Single Click Ensemble Segmentation Approach. Pattern Recognit 46, 692-702 (2013).

30. Aerts, H. J. et al. Identification of residual metabolic-active areas within individual NSCLC tumours using a pre-radiotherapy (18)Fluorodeoxyglucose-PET-CT scan. Radiother Oncol 91, 386-392 (2009). 


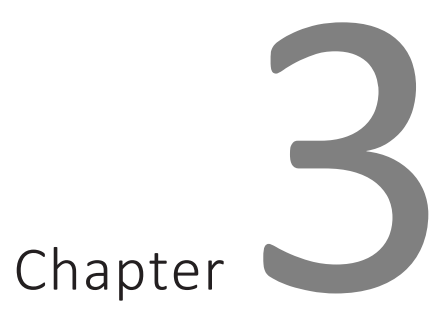

\section{Robust lung nodule segmentation using the 3D-Slicer chest imaging platform}

Submitted: Journal of medical imaging -SPIE

Robust lung nodule segmentation using the 3D Slicer chest imaging platform

Stephen Yip*, Chintan Parmar*, John Kim, Daniel Blezek, Steve Pieper, Hugo J.W.L. Aerts

*These authors contributed equally to this work 


\section{ABSTRACT}

Accurate segmentation of lung nodules is crucial in the development of imaging biomarkers for predicting nodule malignancy. Manual segmentation is time consuming and affected by inter-observer variability. We evaluated the robustness and accuracy of a publically available semiautomatic segmentation algorithm that is implemented in the 3D-Slicer Chest Imaging Platform (CIP) and compared it with the performance of manual segmentation. CT images of 354 manually segmented nodules were downloaded from the LIDC database. Four radiologists performed the manual segmentation and assessed various nodule characteristics. The CIP segmentation was initialized using the center of gravity of the manual segmentations, thereby generating four contours for each nodule. The robustness of both segmentation methods was assessed using the region of uncertainty $(\delta)$ and the Dice similarity index (dsi). The median computational time of the CIP segmentation on a personal computer was only 10s. CIP segmentations were significantly ( $p$ Wilcoxon 10-16) more robust than manual segmentations (median $\delta C I P=14 \mathrm{ml}$, median $(\mathrm{dsiCIP})=99 \%$ vs. median $\delta$ manual $=222 \mathrm{ml}$, median dsimanual $=82 \%$, respectively). The agreement between CIP and manual segmentations had a median value of $60 \%$. CIP segmentation can potentially reduce the physician workload and inter-observer variability owing to its computational efficiency and superior stability compared to manual segmentation. 


\section{INTRODUCTION}

Quantitative imaging has become an important area of research for the development of non-invasive imaging biomarkers for numerous applications, such as the prediction of clinical outcomes, and assessment of treatment response and gene expression ${ }^{1,2}$. In particular, quantitative imaging has the potential to have an immense impact on lung cancer patients. Lung cancer is a leading cause of cancer-related death among men and women, affecting over 1.8 million patients worldwide ${ }^{3}$. At the time of diagnosis, the majority of patients are in advanced stages of disease, resulting in poor prognoses with a 5 -year overall survival rate of $<20 \%{ }^{4}$. However, patients who are treated for early stage disease have a substantially greater overall survival rate of $>50 \%{ }^{4}$. Therefore, identification of patients with early stage disease is crucial for improving prognosis of lung cancer patients ${ }^{5}$.

Computed tomography (CT) is routinely used to diagnose and monitor disease progression in lung cancer patients, where early stage disease is often manifested as pulmonary nodules ${ }^{6,7}$. One of the challenges of identifying patients with early stage lung cancer is that these pulmonary nodules may also be an indicator of other benign conditions, such as inflammation and/or infection, rather than malignancy ${ }^{8}$. Studies have hypothesized that malignant nodules possess distinctive CT imaging features from benign nodules, such as greater lesion volume, longer diameter and faster growth rate ${ }^{9-14}$. Classifiers that are built using imaging features have shown promise in assisting physicians to effectively identify different nodule types ${ }^{15-20}$. The development and accuracy of these classifiers relies on accurate delineation of the region of interest that conforms only to the nodule boundaries. Quantitative imaging features are then extracted and evaluated from this region of interest to generate the classifier. Therefore, inaccurate segmentation of tumors can lead to the development of inaccurate classifiers or biomarkers. Manual segmentation by experienced radiologists is commonly used for defining the nodule volume (or region of interest) using a slice-by-slice approach. However, manual segmentation is not only labor intensive, but is also impacted by inter-and intra-observer variability ${ }^{21-24}$. A number of automatic and semiautomatic segmentation methods have been proposed, ranging from simple approaches, such as thresholding ${ }^{25}$ and region growing ${ }^{26}$, to more complex methods based on the probability map of nodule textures and convexity ${ }^{17,27,28}$. Despite having great potential to reduce human errors and expedite the nodule contouring workflow, these methods are currently not publically accessible, which limits their widespread use in clinical and biomedical research.

Alternatively, 3D Slicer is an open-source software platform for biomedical research 29 that supports versatile visualization and provides advanced analysis tools, such as image segmentation and registration. An algorithm implemented in 3D Slicer, known as GrowCut, can delineate large lung tumor volumes more robustly than manual segmentation ${ }^{30}$, and reliably extract imaging features for the development of imaging biomarkers ${ }^{31}$. However, segmentation of pulmonary nodules presents a unique challenge 
since the nodules are often smaller and in close proximity with surrounding tissues. Therefore, additional pruning steps are required in the nodule segmentation process to remove pleural and/or vessel attachments ${ }^{32,33}$. To address these challenges with nodule segmentation, a level set-based algorithm has been implemented within the Chest Imaging Platform (CIP) in 3D Slicer ${ }^{33,34}$. This algorithm is based on a front propagation approach from a "seed point" placed within the nodule. The propagation of the front (or segmentation) is constrained to prevent leakage into the chest wall, airway walls or regions with appearance of tubular or vessel-like structures.

In this study, we evaluated the robustness of the CIP segmentation algorithm in delineating lung nodules and compared its performance with the manual segmentations. The accuracy of the CIP segmentation algorithm and nodule characteristics that could affect the segmentation quality was also investigated.

\section{METHODS AND MATERIALS}

\section{Patient dataset}

A publicly available thoracic CT dataset, known as the Lung Image Database Consortium (LIDC), was downloaded from The Cancer Imaging Archive (TCIA $)^{23}$. The LIDC dataset consisted of 1007 patients with low dose helical thoracic CT images containing annotated lung nodules that were acquired from seven academic institutions with slice thicknesses ranging from $1 \mathrm{~mm}$ to $5 \mathrm{~mm}$. Each nodule had 1 to 4 manual segmentations that were performed on a slice-by-slice basis by experienced thoracic radiologists. The radiologists assigned scores (ranging from 1 to 5) to each nodule for nine categories that described the nodule characteristics, including its subtlety, internal structure, roundness, margin sharpness, lobulation, spiculation, texture, and likelihood of being malignant. Table 1 contains an annotation of the scoring system. Images were excluded from the current study if they were segmented by less than 4 radiologists ( $n=596)$, nodule numbers were mislabeled ( $n=60$ ) or had imaging artifacts ( $=77$, Figure S1). In total, images from 274 patients with 354 nodules (1-4 nodules/patients) were analyzed.

\section{Nodule segmentation algorithm in 3D Slicer}

The CIP in 3D Slicer $4.5^{29}$ employs a Lesion Sizing Toolkit-based segmentation algorithm for semi-automatic nodule segmentation (Krishnan et al 2010). A seed point is placed within the nodule region to initialize the segmentation. In this current study, the seed point was chosen as the center of gravity of each manual contour. Therefore, four CIPsegmentations were generated automatically for each nodule.

Using the level set method ${ }^{35}$, nodule segmentation was propagated outward from the seed point until the propagation was retarded by encountering boundaries, such as the airway, chest wall, vasculature, or other enhanced edges that were not nodules. 
The segmentation algorithm automatically generated boundaries. For example, the segmentation algorithm penalized regions that had the appearance of tubular or vessel-like structures using Hessian characteristics ${ }^{36}$. Furthermore, the maximum radius of the nodule from the seed point was set to $30 \mathrm{~mm}$.

\section{Analysis}

\section{Robustness of the segmentation methods}

The region of uncertainty $(\delta)$ and dice similarity index (DSI), were used to assess the robustness of the manual and CIP segmentations. The region of uncertainty was defined as the negation of the intersect regions of all the segmentations (Figure 1). In particular, the region of uncertainty $(\delta)$ was defined as follow:

$$
\delta_{\text {method }}=\bigcap V_{\text {method }}^{I} \bigcap V_{\text {method }}^{I I} \bigcap V_{\text {method }}^{\text {III }} \bigcap V_{\text {method }}^{I V}
$$

Where method could either be manual or CIP segmentation. For manual segmentation, the superscript indicates the nodule volume delineated by the four different radiologists, whereas for CIP segmentation, it indicates the segmentations initialized by the center of gravity computed from the four radiologist-defined volumes. $\delta$ equaled to zero indicated that the segmentation method was perfectly robust across the four segmentations. The stability of the segmentation method decreases with increasing in the $\delta_{\text {method }}$.

The DSI for segmentation stability was defined as follow:

$$
d s i_{\text {method }}=\frac{1}{6} \sum_{i \neq j} \frac{V_{\text {method }}^{i} \cap V_{\text {method }}^{j}}{V_{\text {method }}^{i} \cup V_{\text {method }}^{j}} \cdot 200 \%
$$

Where method could either be manual or CIP segmentation. $i$ and $j$ ranged from 1 to 4 indicating nodule volumes segmented by radiologist $i$ and $j$, or initialized by the center of gravity computed from radiologist $i$ and $j$, for manual and CIP segmentations, respectively. There were four contours for each segmentation method and, thus, six possible combinations of $i$ and $j$. The stability of the segmentation method increases with increasing $\mathrm{dsi} \mathrm{i}_{\text {method }}$, where $\mathrm{dsi} \mathrm{i}_{\text {method }}=100 \%$ indicates a perfectly robust method.

The robustness of the CIP segmentation method $\left(\delta_{\mathrm{CIP}}\right.$ or $\left.\mathrm{dsi} \mathrm{i}_{\mathrm{CIP}}\right)$ was compared with the manual segmentation method ( $\delta_{\text {manual }}$ or $d \mathrm{di}_{\text {manual }}$ ) using the Wilcoxon signed-rank test, where $p_{\text {wilcoxon }}<0.05$ indicated statistical significance. Moreover, the average nodule volume segmented by the manual and CIP contouring methods were also compared and tested for significant differences. The average nodule volume was defined as $\overline{V_{\text {method }}}=\frac{1}{4} \sum_{i=1}^{4} V_{\text {method }}^{i}$; where $i$ indicates radiologist $i$. Since the ground truth of the nodule segmentation is unknown, the average nodule volume $\left(\overline{V_{\text {manual }}}\right)$ computed from the manual contours was used to estimate the true nodule volume. Unless otherwise specified, $\overline{V_{\text {manual }}}$ is referred to as nodule volume. 


\section{Accuracy of the CIP segmentation method}

The accuracy of CIP segmentations was evaluated to ensure that non-nodular tissues were excluded and the entire nodule volume was contoured. Even if the CIP segmentation was perfectly robust, it may include nearby non-nodule tissues or fail to capture the entire nodule region. For example, despite being almost perfectly robust, nodules contoured by the CIP segmentation method were observed to include substantial normal lung regions as shown in Figure $3 \mathrm{a}$ and $3 \mathrm{~b}$. The agreement between the manual and CIP segmentations was used to estimate how well the nodule volume could be delineated by the CIP segmentation. DSI Agree $_{\text {was }}$ used to assess for the segmentation agreement and was defined as follow:

$$
D S I_{\text {Agree }}=\frac{1}{4} \sum_{i=1}^{4} \frac{V_{\text {manual }}^{i} \cap V_{C I P}^{i}}{V_{\text {manual }}^{i} \cup V_{C I P}^{i}} \cdot 200 \%
$$

Where $V_{\text {manual }}^{i}$ indicates the nodule volume segmented by radiologist $i$ while $V_{C I P}^{i}$ is the CIP segmentation nodule volume initialized by the center of gravity of $V_{\text {manual }}^{i}$.

To avoid confusion, lower case dsi was used to indicate the robustness of the segmentation method while upper case DSI was used to indicate the accuracy of the CIP segmentation in this paper.

\section{Relationship between nodule characteristics and CIP segmentation accuracy}

To identify nodule characteristics that may affect the accuracy of the CIP segmentation, the Spearman's correlation coefficient was computed between the radiologists scored nodule characteristics and $\mathrm{DSI}_{\mathrm{Agree}}$. For nodule characteristics that had a continuous scoring scale (e.g. margin ranges from 1 to 5, where 1 indicates a poorly defined margin and 5 indicates a sharp margin) (Table 1), a t-test was used to assess if the correlation coefficient was significantly different from $0\left(p_{t-t e s t}<0.05\right)$. For characteristic categories where the scoring scale was categorical rather than continuous (i.e. nodule calcification where each score indicates a different appearance) (Table 1), the Kruskal-Wallis test $\left(p_{\text {Kruskal-Wallis }}<0.05\right)$ was used.

The correlations between $\overline{V_{\text {manual }}}, \mathrm{DSI}_{\text {Agree }}$ and all nodule characteristics were also calculated. Four radiologists scored each category, and thus, there was some variability in the characteristic scoring. When there was a heterogeneous rating, the score that was assigned by the majority of radiologists was chosen for the analysis. In the case of a tie rating, the score that were most frequently assigned to the patient population was chosen. The distributions of the scores for each nodule characteristic are shown in supplementary Figure S1. 


\section{RESULTS}

In this study, a semiautomatic segmentation method implemented in the CIP of 3D Slicer was used to contour 354 nodules. The computation time of the CIP segmentations was 5-79 s (median: 10s) on a personal computer with 16GB RAM and 3.40GHz Core i74770 CPU.

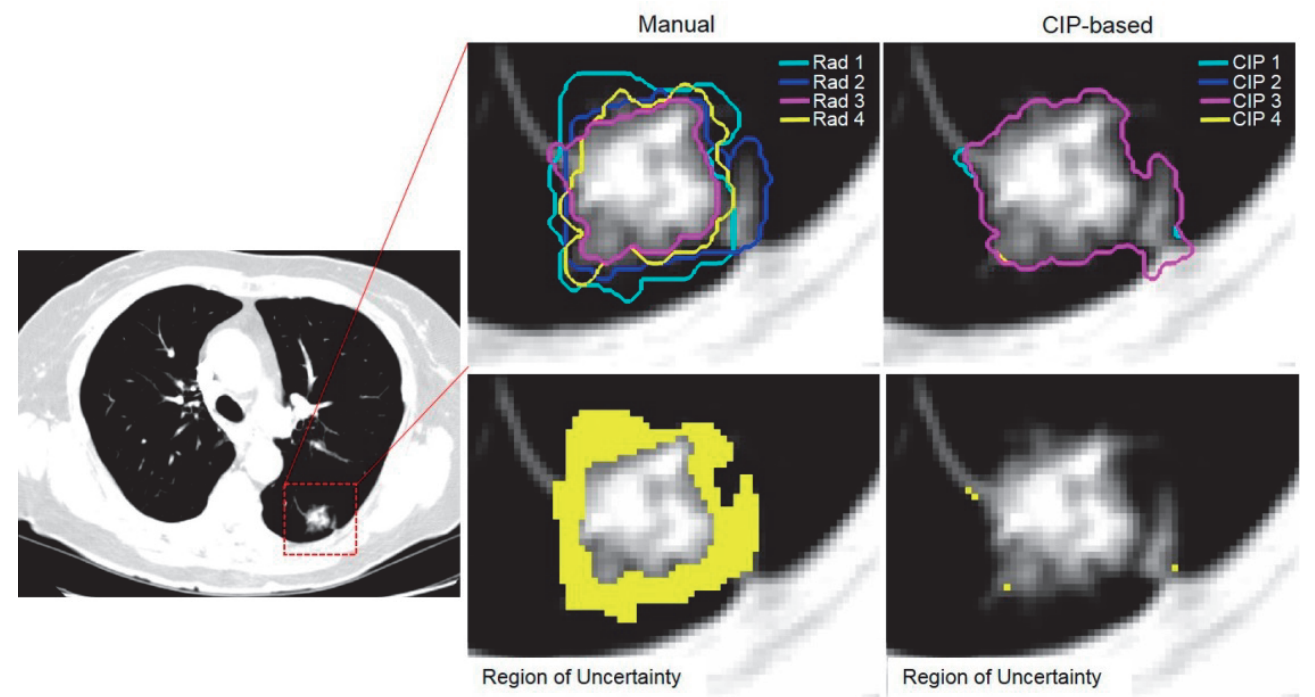

Figure 1. Comparison of manual (left) and CIP (right) segmentation. Yellow shaded region indicated the disagreement (or region of uncertainty) between contours performed by four radiologists (bottom left) or different CIP seed locations (bottom right). In this example, the region of uncertainty for manual segmentation was $3222 \mathrm{ml}$ while the region was only $46 \mathrm{ml}$ for the CIP segmentation. dsi $\mathrm{CIP}_{\mathrm{P}}$ was $\approx 100 \%$, while dsi manual was $88 \%$.

\section{Robustness of the segmentation methods}

For the CIP segmentation method, the median dsi IIP $_{\text {was }}$ 99\% (Interquartile (IQR) range: 97-100\%) and the median $\delta_{\mathrm{CIP}}$ was $14 \mathrm{ml}$ (IQR range: $7-37 \mathrm{ml}$ ), while for the manual segmentation method, dsi manual was $82 \%$ (IQR range: $77-85 \%$ ) and the median $\delta_{\text {manual }}$ was $222 \mathrm{ml}$ (IQR range: $124-461 \mathrm{ml}$ ). Although both segmentation methods were generally robust (median dsi>80\%), CIP segmentations were significantly more stable than the manual segmentations with $p_{\text {Wilcoxon }} \sim 10^{-16}$ for both robustness measures. Figure $3 a$ shows a visual example of a patient with more stable nodule contours by the CIP segmentation method than by the manual segmentation method. 

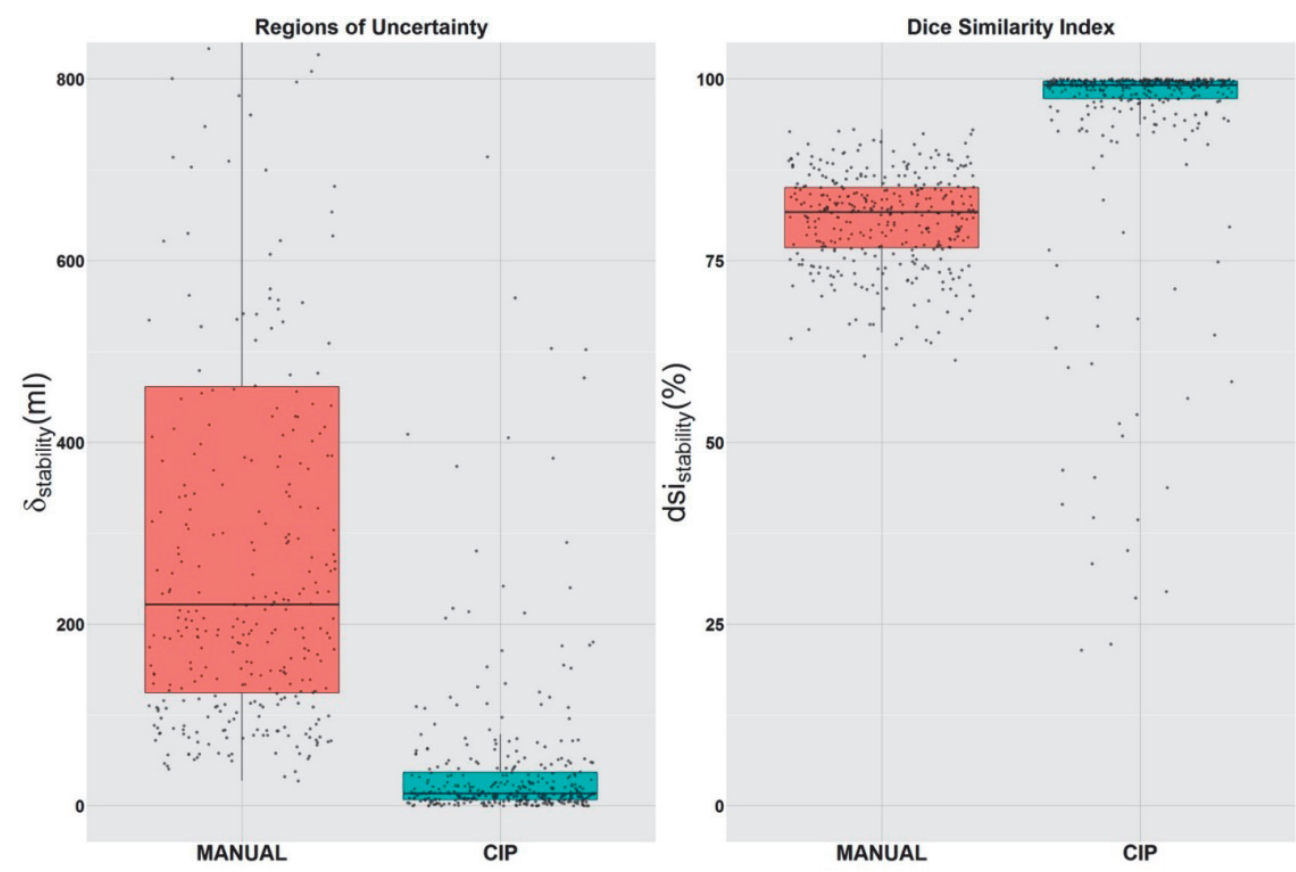

Figure 2. Robustness (or Stability) of the manual and CIP segmentation assessed with the region of uncertainty $(\delta)$ and Dice similarity index (dsi).

\section{Accuracy of the CIP segmentation}

The median value of $\overline{V_{\text {manual }}}$ was $309 \mathrm{ml}$ (IQR range: $162-796 \mathrm{ml}$ ) and $\overline{V_{C I P}}$ was $477 \mathrm{ml}$ (IQR range: $153-1290 \mathrm{ml}$ ). Nodules segmented by the CIP method were significantly greater in volume than those by manual method $\left(p_{\text {Wilcoxon }} \sim 10^{-12}\right)$. Figure $3 b$ shows an example where CIP segmentation overestimated the nodule region, including parts of the normal lung.

The agreement between CIP and manual segmentations that was assessed by the median DSI was 60\% (IQR range: 46-71\%). The relationship between various nodules

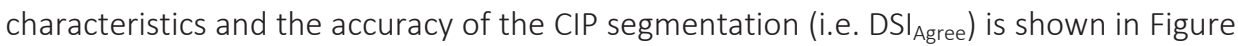
4. Nodule subtlety, margin, texture, lobulation, malignancy, and nodule volume $\left(\overline{V_{\text {manual }}}\right)$ were positively and significantly correlated to the $\mathrm{DSI}_{\text {Agree }}$ ( $\mathrm{p}_{\mathrm{t} \text {-test }}$ range: $1.5 \times 10^{-9}-6 \times 10^{-3}$ ) (Figure 4). As the nodule volume increased from $162 \mathrm{ml}$ to $796 \mathrm{ml}$, the

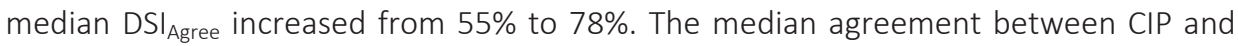
manual segmentations increased from $56 \%$ to $70 \%$ as the likelihood of the nodule malignancy increased. 

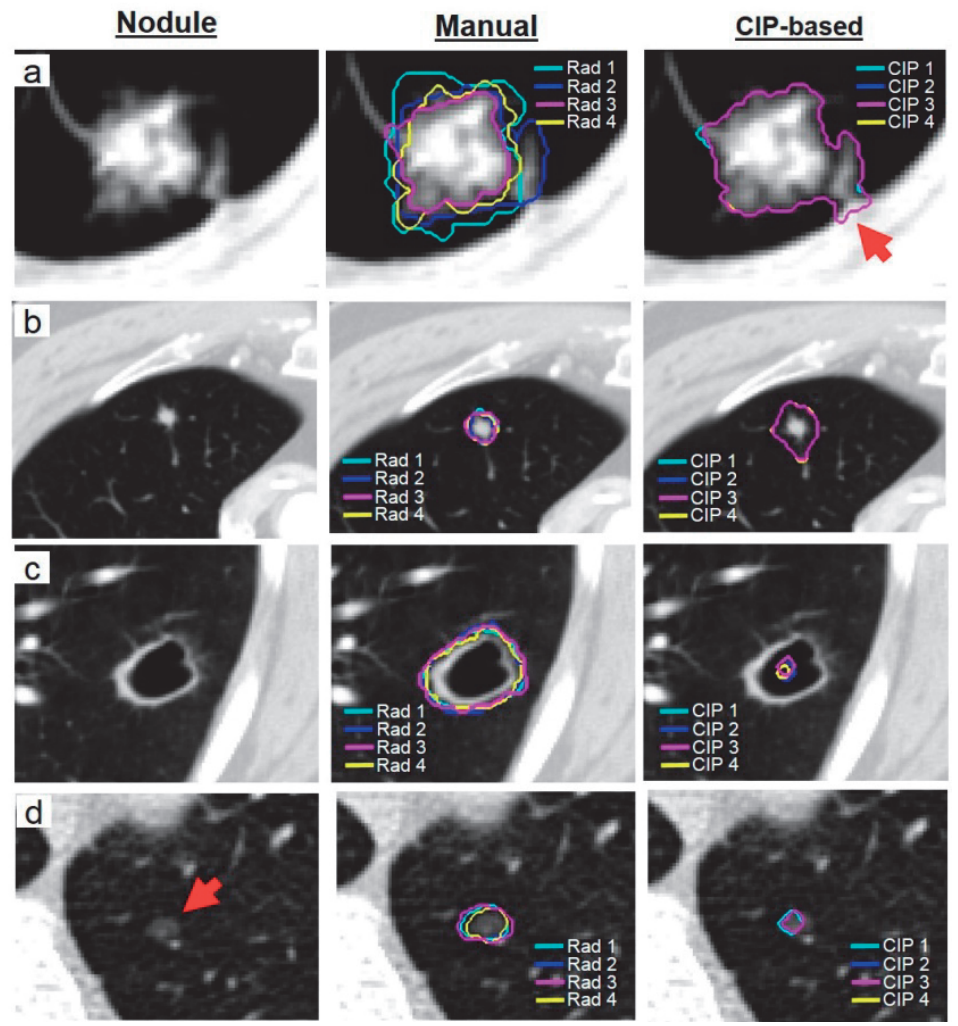

Figure 3. Examples of nodules that were segmented by radiologists manually and CIP segmentations. a) The robustness of the CIP segmentation was excellent, while substantial interobserver variability was observed in manual segmentation. CIP segmentation was also in excellent agreement with manual contours. However, CIP segmentation was observed to include part of the chest wall (indicated by an arrow) b) Despite being perfectly robust CIP segmentation, it included the region of the normal lung in proximity of the small nodule. c) Cavitation in the center of the nodule. Poor CIP segmentation performance was found. d) Non-solid (ground glass opacity) nodule with poorly defined boundary and subtle appearance is indicated by the red arrow. Poor CIP segmentation performance was found.

An example of a non-solid subtle nodule with poorly defined boundaries is shown in Figure $3 \mathrm{~d}$. The accuracy of the CIP segmentation was poor for non-solid or semi-solid nodules, or nodules with poorly defined boundaries and subtle appearances with a median $\mathrm{DSI}_{\text {Agree }}$ ranging from $15 \%-41 \%$. The performance of CIP segmentations for solid nodules with sharp margins and obvious appearances increased to $61 \%$. Nodules that were not marked to be lobulated or spiculated by the radiologists had a median $\mathrm{DSI}_{\text {Agree }}$ of 59\%. Substantial agreement (median DSI Agree $>70 \%$ ) between CIP and manual segmentations were found in nodules with marked lobulation and spiculation. Nodule sphericity $\left(p_{\text {t-test }}=0.94\right)$ and calcification $\left(p_{\text {rruskal-Wallis }}=0.49\right.$ ) were not significantly correlated with $\overline{V_{\text {manual }}}$. Median DSI $\mathrm{Agree}_{\text {was }} \sim 60 \%$ for all nodules regardless of the nodule sphercitiy and calcification conditions (Figure 4). 

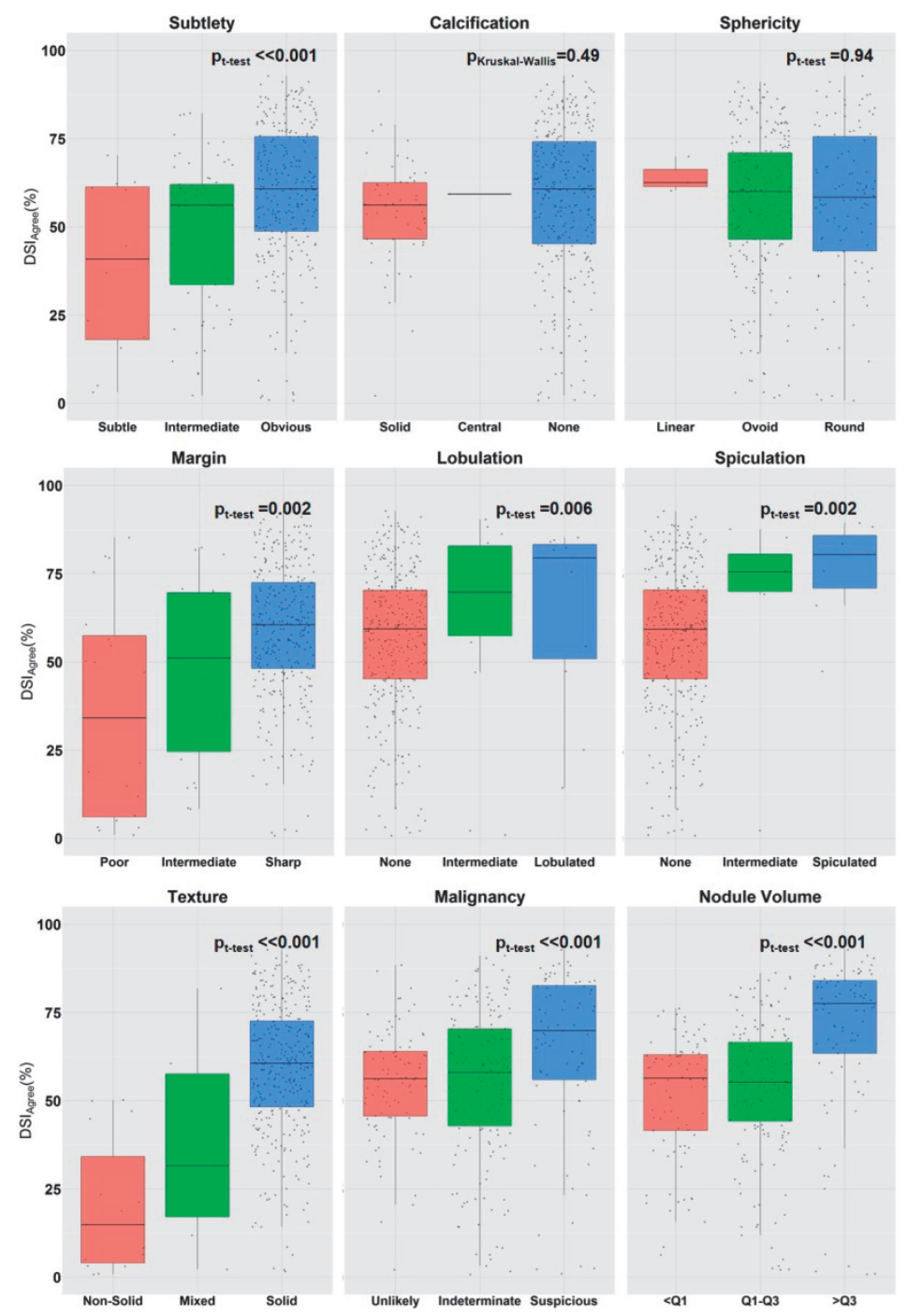

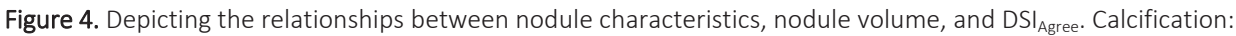
Solid=solid calcification, Central=central calcification, None=no calcification. Lobulation: None=not lobulated. Spiculated: None=not spiculated. Texture: Mixed=Semi-solid nodules. Malignancy: Unlikely= unlikely for cancer, Suspicious=suspicious for cancer. Nodule Volume: Q1=162ml, Q1-Q3=162ml to 796ml, and Q3=796ml; Q= quantile.

While the interior structure of all the other 343 nodules was scored as soft tissue, one nodule was rated to be air (Figure 3c). For this nodule, the CIP segmentation failed to identify the boundary of the nodule resulting in a $\mathrm{DSI}_{\text {Agree. }}$ of $1 \%$ and was unstable $\left(\mathrm{dsi}_{\mathrm{CIP}}=42 \%\right.$ ) (Figure $3 \mathrm{c}$ ). Nodule malignancy, subtlety, calcification, lobulation, and spiculation were positively and significantly correlated to $\overline{V_{\text {manual }}}\left(\mathrm{p}_{\text {t-test }}\right.$ range $=6.87 \times 10^{-}$ $\left.{ }^{27}-1.12 \times 10^{-4}\right)$. 


\section{DISCUSSION}

Pulmonary nodules can indicate early stage lung cancer or a number of benign conditions. CT-based imaging features have been used to generate imaging biomarkers that predict the malignancy of lung nodules and have demonstrated promising results ${ }^{19,20}$. Careful delineation of the lung nodule volumes is required for accurate feature extraction to build these imaging biomarkers ${ }^{15-18}$. Most commonly, manual segmentation is the method of choice; however, manual segmentation is not only time consuming, but is also affected by inter-observer variability ${ }^{21,22,24}$. Although many automatic and semiautomatic segmentation algorithms for nodule segmentation have been proposed, the widespread use of these algorithms, in the scientific and clinical communities, are hampered by their limited accessibility. In this study, we compared the robustness of manual segmentation and a publically accessible nodule segmentation algorithm, known as CIP segmentation.

CIP segmentation may provide a reliable way to assist physicians in the nodule delineation process by reducing inter-observer variability and the physician workload. The CIP segmentations computed from different seed points from the four radiologists were in excellent agreement, indicating that the CIP method is robust to different segmentation seed points. In comparison, manual segmentation was significantly less stable than CIP segmentation. Comparatively, Velazquez et al (2013) assessed the robustness of manual delineations and a 3D Slicer semi-automatic algorithm, known as GrowCut, in defining the volume of twenty non-small cell lung (NSCLC) tumors ${ }^{30}$. They found that the GrowCut algorithm resulted in significantly smaller regions of uncertainty than manual delineations and concluded that it could be used as a starting point for tumor target delineation in radiotherapy and high-throughput data mining research when manual delineations are not available. The results of our study are consistent with their findings that semiautomatic algorithms (in our case, CIP segmentations) are more stable than manual segmentations in defining lung nodule volumes.

An emerging field that converts medical images into high dimensional mineable data is called radiomics ${ }^{37}$. In addition to differentiating between benign and malignant nodules, radiomic features of lung lesions could also be used to predict clinical outcomes $^{1,2,38}$. Several lung screening trials using CT images have been launched in $\mathrm{Asia}^{39-}$ ${ }^{41}$, Europe ${ }^{42-44}$, and the United States ${ }^{5,45,46}$ to identify patients with early lung cancer. Due to the easy accessibility of the CIP segmentation algorithm, this method may be useful for nodule delineation in these lung trial datasets that consist of a large number of patients. This could subsequently expedite the high-throughput extraction of imaging features for radiomic analysis for nodule classification and patient outcomes.

Despite the potential applications of the CIP segmentation algorithm, manual adjustment of the segmentations may be needed, especially for small nodules and nodules with poorly defined boundaries, subtle appearance, and non-solid or part-solid textures. Nodule calcification and sphericity have no impact on the performance of CIP segmentations. The accuracy of CIP segmentations tended to be better when the nodule was 
solid, more obvious, and with a sharp boundary. Non- and part-solid nodules with a hazy appearance failed to completely obscure parenchymal structures, and have been therefore difficult detect and segment by many segmentation algorithms ${ }^{27,28,47,48}$. Similarly, CIP segmentations also suffer from this limitation, where nodules with subtle appearances may have similar image intensity as its background that makes the full extent of nodules difficult to define. Therefore, in these cases, the knowledge of experienced radiologists is needed to estimate the extent (or boundary) of the nodules and manually edited the CIP segmentation. Several segmentation algorithms have been proposed to improve the contours of structures with hazy appearances, such as non- and part-solid nodules, such as the Markov random field theory-based algorithm ${ }^{49,50}$ and a hybrid algorithm that combines threshold-based region growing, connected component analyses and convex hull calculations ${ }^{27,28,47,48,51}$. However, these more sophisticated algorithms are not easily accessible and should be implemented into open source platform for widespread use. In particular, incorporating algorithms for defining non- and subsolid nodules into the 3D Slicer CIP can further improve the performance of the CIP segmentations.

CIP segmentations may overestimate nodule region of interest for small nodules. CIP segmentations performed better for nodules with larger volumes $\left(\overline{V_{\text {manual }}}\right)$ and a higher likelihood of being malignant. Nodules that are larger in size (e.g. $>4 \mathrm{~mm}$ nodule diameter in the National Lung Screening Trail in the United Stated ${ }^{5}$ ) are generally considered to be more likely to be malignant. Moreover, the appearance of a large nodule is less subtle and more obvious. As expected, in our study, nodule malignancy and subtlety were positively correlated with nodule volumes. Excellent agreement between CIP and manual segmentations were found for nodule volumes $>796 \mathrm{ml}$. Moreover, larger nodule volumes may be more likely to be lobulated and spiculated due to the significant correlation between these characteristics and the nodule volume. This may explain why the CIP segmentation method performed better for nodules with marked lobulation and spiculation. We observed that nodule volumes computed from CIP segmentations were significantly greater than those computed from manual segmentation. For nodules with smaller size, CIP segmentations often include adjacent tissues, such as normal lung and blood vessels. Furthermore, small nodules were not only more likely to have subtle appearances and thus, were difficult to detect, but could also be easily overestimated by CIP segmentations. Therefore, manual adjustments may be needed to correct for the overestimation of the small nodules in the CIP segmentations.

\section{CONCLUSION}

A semi-automatic segmentation algorithm implemented under the 3D Slicer Chest Imaging Platform (CIP) may be useful for assisting physicians in nodule volume delineation. CIP segmentations can potentially reduce the physician workload and inter-observer variability due to its computational efficiency and superior stability compared to manual 
segmentation. Due to the public accessibility of the CIP segmentation algorithm, it can be employed to initiate nodule segmentation for large datasets, such as lung screening trials, thereby facilitating efficient nodule classification and high-throughput data mining research. However, CIP segmentations should be used with care and manual adjustment of the segmentations may be needed for small nodules, and nodules with subtle appearances, poorly defined boundaries and non- and part-solid texture.

\section{TABLES}

Table 1. Annotation of nodule characteristic scoring. The intermediate values for Roundness, Margin, Lobulation, Spiculation, and Texture are allowed to use by the radiologists.

\begin{tabular}{|c|c|c|c|c|c|c|}
\hline $\begin{array}{l}\text { Nodule } \\
\text { Characteristics }\end{array}$ & Level-1 & Level-2 & Level-3 & Level-4 & Level-5 & Level-6 \\
\hline Subtlety & $\begin{array}{l}\text { Extremely } \\
\text { Subtle }\end{array}$ & $\begin{array}{l}\text { Moderately } \\
\text { Subtle }\end{array}$ & Fairly Subtle & $\begin{array}{l}\text { Moderately } \\
\text { Obvious }\end{array}$ & Obvious & N/A \\
\hline $\begin{array}{l}\text { Internal } \\
\text { Structure }\end{array}$ & $\begin{array}{l}\text { Soft Tissue } \\
\text { (default) }\end{array}$ & Fluid & Fat & Air & N/A & N/A \\
\hline Calcification & Popcorn & Laminated & Solid & Non-Central & Central & Absent \\
\hline $\begin{array}{l}\text { Sphericity } \\
\text { (Roundness) }\end{array}$ & Linear & & Ovoid & & Round & \\
\hline Margin & Poorly & & & & Sharp & N/A \\
\hline Lobulation & None & & & & Marked & N/A \\
\hline Spiculation & None & & & & Marked & N/A \\
\hline Texture & $\begin{array}{l}\text { Non- } \\
\text { Solid/Ground } \\
\text { Glass Opacity }\end{array}$ & & Part Solid/Mixed & & Solid & N/A \\
\hline Malignancy & Highly unlikely & $\begin{array}{l}\text { Moderately } \\
\text { unlikely }\end{array}$ & Indeterminate & $\begin{array}{l}\text { Moderately } \\
\text { suspicious }\end{array}$ & $\begin{array}{l}\text { Highly } \\
\text { suspicious }\end{array}$ & N/A \\
\hline
\end{tabular}

\section{ACKNOWLEDGEMENTS}

The authors would like to acknowledge support from the National Institute of Health (Award Number U01CA190234 and U24CA194354) and research seed funding grant from the American Association of Physicists in Medicine. Furthermore, we would like to thank Dr. Elizabeth Huynh for editorial assistance. 


\section{REFERENCES}

1 H. J. W. L. Aerts, E. R. Velazquez, R. T. H. Leijenaar, C. Parmar, P. Grossmann, S. Carvalho, J. Bussink, R. Monshouwer, B. Haibe-Kains, D. Rietveld, F. Hoebers, M. M. Rietbergen, C. R. Leemans, A. Dekker, J. Quackenbush, R. J. Gillies, and P. Lambin, "Decoding tumour phenotype by noninvasive imaging using a quantitative radiomics approach," Nat Commun 5, 4006 (2014).

2 T. P. Coroller, V. Agrawal, V. Narayan, Y. Hou, P. Grossmann, S. W. Lee, R. H. Mak, and H. J. W. L. Aerts, "Radiomic phenotype features predict pathological response in non-small cell lung cancer," Radiotherapy and Oncology 119, 480-486 (2016).

3 L. A. Torre, F. Bray, R. L. Siegel, J. Ferlay, J. Lortet-Tieulent, and A. Jemal, "Global cancer statistics, 2012," CA: A Cancer Journal for Clinicians 65, 87-108 (2015).

4 A. L. Association, "Lung Cancer Fact Sheet," http://www.lung.org/ (2016).

5 T. N. L. S. T. R. Team, "Reduced Lung-Cancer Mortality with Low-Dose Computed Tomographic Screening," New England Journal of Medicine 365, 395-409 (2011).

6 "World Health Organization. WHO methods and data sources for global burden of disease estimates 2000-2011. World Health Organization. 2013. http://www.who.int/healthinfo/statistics/ GlobalDALYmethods_2000_2011.pdf?ua=1. Accessed 13 Aug 2015."

7 S. G. Armato, M. L. Giger, C. J. Moran, J. T. Blackburn, K. Doi, and H. MacMahon, "Computerized Detection of Pulmonary Nodules on CT Scans," RadioGraphics 19, 1303-1311 (1999).

8 J. J. Erasmus, J. E. Connolly, H. P. McAdams, and V. L. Roggli, "Solitary Pulmonary Nodules: Part I. Morphologic Evaluation for Differentiation of Benign and Malignant Lesions," RadioGraphics 20, 43-58 (2000).

9 M. F. McNitt-Gray, N. Wyckoff, J. W. Sayre, J. G. Goldin, and D. R. Aberle, "The effects of co-occurrence matrix based texture parameters on the classification of solitary pulmonary nodules imaged on computed tomography," Computerized Medical Imaging and Graphics 23, 339-348 (1999).

10 S. K. Shah, M. F. McNitt-Gray, S. R. Rogers, J. G. Goldin, R. D. Suh, J. W. Sayre, I. Petkovska, H. J. Kim, and D. R. Aberle, "Computer Aided Characterization of the Solitary Pulmonary Nodule Using Volumetric and Contrast Enhancement Features1," Academic Radiology 12, 1310-1319 (2005).

11 F. Li, S. Sone, H. Abe, H. MacMahon, and K. Doi, "Malignant versus Benign Nodules at CT Screening for Lung Cancer: Comparison of Thin-Section CT Findings," Radiology 233, 793-798 (2004).

12 M.-P. Revel, A. Merlin, S. Peyrard, R. Triki, S. Couchon, G. Chatellier, and G. Frija, "Software Volumetric Evaluation of Doubling Times for Differentiating Benign Versus Malignant Pulmonary Nodules," American Journal of Roentgenology 187, 135-142 (2006).

13 D. F. Yankelevitz, A. P. Reeves, W. J. Kostis, B. Zhao, and C. I. Henschke, "Small Pulmonary Nodules: Volumetrically Determined Growth Rates Based on CT Evaluation," Radiology 217, 251-256 (2000).

14 Y. H. Lee, D. W. Kim, H. S. In, J. S. Park, S. H. Kim, J. W. Eom, B. Kim, E. J. Lee, and M. H. Rho, "Differentiation between Benign and Malignant Solid Thyroid Nodules Using an US Classification System," Korean Journal of Radiology 12, 559-567 (2011).

15 A. Farag, A. Ali, J. Graham, S. Elhabian, A. Farag, and R. Falk, in Advances in Visual Computing: 6th International Symposium, ISVC 2010, Las Vegas, NV, USA, November 29 - December 1, 2010, Proceedings, Part III, edited by G. Bebis, R. Boyle, B. Parvin, D. Koracin, R. Chung, R. Hammound, M. Hussain, T. KarHan, R. Crawfis, D. Thalmann, D. Kao, and L. Avila (Springer Berlin Heidelberg, Berlin, Heidelberg, 2010), p. $79-88$.

16 S. G. Armato, M. B. Altman, J. Wilkie, S. Sone, F. Li, K. Doi, and A. S. Roy, "Automated lung nodule classification following automated nodule detection on CT: A serial approach," Medical Physics 30, 1188-1197 (2003).

17 A. K. Dhara, S. Mukhopadhyay, A. Dutta, M. Garg, and N. Khandelwal, "A Combination of Shape and Texture Features for Classification of Pulmonary Nodules in Lung CT Images," J Digit Imaging 29, 466-475 (2016).

18 H. Madero Orozco, O. O. Vergara Villegas, V. G. Cruz Sánchez, H. d. J. Ochoa Domínguez, and M. d. J. Nandayapa Alfaro, "Automated system for lung nodules classification based on wavelet feature descriptor and support vector machine," BioMedical Engineering OnLine 14, 1-20 (2015). 
19 S. Hawkins, H. Wang, Y. Liu, A. Garcia, O. Stringfield, H. Krewer, Q. Li, D. Cherezov, R. A. Gatenby, Y. Balagurunathan, D. Goldgof, M. B. Schabath, L. Hall, and R. J. Gillies, "Predicting malignant nodules from screening CTs," Journal of Thoracic Oncology (2016).

20 J. Ma, Q. Wang, Y. Ren, H. Hu, and J. Zhao, in "Automatic lung nodule classification with radiomics approach, 2016, p. 978906-978906-6.

21 B. d. Hoop, H. Gietema, S. v. d. Vorst, K. Murphy, R. J. v. Klaveren, and M. Prokop, "Pulmonary GroundGlass Nodules: Increase in Mass as an Early Indicator of Growth," Radiology 255, 199-206 (2010).

22 J. K. Leader, T. E. Warfel, C. R. Fuhrman, S. K. Golla, J. L. Weissfeld, R. S. Avila, W. D. Turner, and B. Zheng, "Pulmonary Nodule Detection with Low-Dose CT of the Lung: Agreement Among Radiologists," American Journal of Roentgenology 185, 973-978 (2005).

23 S. G. Armato, G. McLennan, L. Bidaut, M. F. McNitt-Gray, C. R. Meyer, A. P. Reeves, B. Zhao, D. R. Aberle, C. I. Henschke, E. A. Hoffman, E. A. Kazerooni, H. MacMahon, E. J. R. van Beek, D. Yankelevitz, A. M. Biancardi, P. H. Bland, M. S. Brown, R. M. Engelmann, G. E. Laderach, D. Max, R. C. Pais, D. P.-Y. Qing, R. Y. Roberts, A. R. Smith, A. Starkey, P. Batra, P. Caligiuri, A. Farooqi, G. W. Gladish, C. M. Jude, R. F. Munden, I. Petkovska, L. E. Quint, L. H. Schwartz, B. Sundaram, L. E. Dodd, C. Fenimore, D. Gur, N. Petrick, J. Freymann, J. Kirby, B. Hughes, A. Vande Casteele, S. Gupte, M. Sallam, M. D. Heath, M. H. Kuhn, E. Dharaiya, R. Burns, D. S. Fryd, M. Salganicoff, V. Anand, U. Shreter, S. Vastagh, B. Y. Croft, and L. P. Clarke, "The Lung Image Database Consortium (LIDC) and Image Database Resource Initiative (IDRI): A Completed Reference Database of Lung Nodules on CT Scans," Medical Physics 38, 915-931 (2011).

24 R. J. H. M. Steenbakkers, J. C. Duppen, I. Fitton, K. E. I. Deurloo, L. J. Zijp, E. F. I. Comans, A. L. J. Uitterhoeve, P. T. R. Rodrigus, G. W. P. Kramer, J. Bussink, K. De Jaeger, J. S. A. Belderbos, P. J. C. M. Nowak, M. van Herk, and C. R. N. Rasch, "Reduction of observer variation using matched CT-PET for lung cancer delineation: A three-dimensional analysis," International Journal of Radiation Oncology*Biology*Physics 64, 435-448 (2006).

25 J. M. Goo, T. Tongdee, R. Tongdee, K. Yeo, C. F. Hildebolt, and K. T. Bae, "Volumetric Measurement of Synthetic Lung Nodules with Multi-Detector Row CT: Effect of Various Image Reconstruction Parameters and Segmentation Thresholds on Measurement Accuracy," Radiology 235, 850-856 (2005).

26 J. Dehmeshki, H. Amin, M. Valdivieso, and X. Ye, "Segmentation of Pulmonary Nodules in Thoracic CT Scans: A Region Growing Approach," IEEE Transactions on Medical Imaging 27, 467-480 (2008).

27 Y. Tao, L. Lu, M. Dewan, A. Y. Chen, J. Corso, J. Xuan, M. Salganicoff, and A. Krishnan, in Medical Image Computing and Computer-Assisted Intervention - MICCAI 2009: 12th International Conference, London, UK, September 20-24, 2009, Proceedings, Part II, edited by G.-Z. Yang, D. Hawkes, D. Rueckert, A. Noble, and C. Taylor (Springer Berlin Heidelberg, Berlin, Heidelberg, 2009), p. 715-723.

28 B. C. Lassen, C. Jacobs, J. M. Kuhnigk, B. v. Ginneken, and E. M. v. Rikxoort, "Robust semi-automatic segmentation of pulmonary subsolid nodules in chest computed tomography scans," Physics in Medicine and Biology 60, 1307 (2015).

29 A. Fedorov, R. Beichel, J. Kalpathy-Cramer, J. Finet, J.-C. Fillion-Robin, S. Pujol, C. Bauer, D. Jennings, F. Fennessy, M. Sonka, J. Buatti, S. Aylward, J. V. Miller, S. Pieper, and R. Kikinis, "3D Slicer as an Image Computing Platform for the Quantitative Imaging Network," Magnetic resonance imaging 30, 1323-1341 (2012).

30 E. R. Velazquez, C. Parmar, M. Jermoumi, R. H. Mak, A. van Baardwijk, F. M. Fennessy, J. H. Lewis, D. De Ruysscher, R. Kikinis, P. Lambin, and H. J. W. L. Aerts, "Volumetric CT-based segmentation of NSCLC using 3D-Slicer," Sci. Rep. 3, 3529 (2013).

31 C. Parmar, E. Rios Velazquez, R. Leijenaar, M. Jermoumi, S. Carvalho, R. H. Mak, S. Mitra, B. U. Shankar, R. Kikinis, B. Haibe-Kains, P. Lambin, and H. J. W. L. Aerts, "Robust Radiomics Feature Quantification Using Semiautomatic Volumetric Segmentation," PLoS ONE 9, e102107 (2014).

32 J. M. Kuhnigk, V. Dicken, L. Bornemann, A. Bakai, D. Wormanns, S. Krass, and H. O. Peitgen, "Morphological segmentation and partial volume analysis for volumetry of solid pulmonary lesions in thoracic CT scans," IEEE Transactions on Medical Imaging 25, 417-434 (2006).

33 K. Krishnan, L. Ibanez, W. D. Turner, J. Jomier, and R. S. Avila, "An open-source toolkit for the volumetric measurement of CT lung lesions," Opt. Express 18, 15256-15266 (2010).

34 E. Raul San Jose, C. R. James, H. Rola, O. Jorge, A. D. Alejandro, and R. W. George, in C66. LUNG IMAGING II: NEW PROBES AND EMERGING TECHNOLOGIES (American Thoracic Society, 2015), p. A4975-A4975. 
35 V. Caselles, R. Kimmel, and G. Sapiro, "Geodesic Active Contours," International Journal of Computer Vision 22, 61-79 (1997).

36 J. C. Ross, R. S. J. Estépar, A. Díaz, C.-F. Westin, R. Kikinis, E. K. Silverman, and G. R. Washko, "Lung Extraction, Lobe Segmentation and Hierarchical Region Assessment for Quantitative Analysis on High Resolution Computed Tomography Images," Medical image computing and computer-assisted intervention : MICCAI ... International Conference on Medical Image Computing and Computer-Assisted Intervention 12, 690-698 (2009).

37 Y. Sato, S. Nakajima, N. Shiraga, H. Atsumi, S. Yoshida, T. Koller, G. Gerig, and R. Kikinis, "Threedimensional multi-scale line filter for segmentation and visualization of curvilinear structures in medical images," Medical Image Analysis 2, 143-168 (1998).

38 S. F. S. Yip and J. W. L. H. Aerts, "Applications and limitations of radiomics," Physics in Medicine and Biology 61, R150 (2016).

39 T. P. Coroller, P. Grossmann, Y. Hou, E. Rios Velazquez, R. T. H. Leijenaar, G. Hermann, P. Lambin, B. Haibe-Kains, R. H. Mak, and H. J. W. L. Aerts, "CT-based radiomic signature predicts distant metastasis in lung adenocarcinoma," Radiotherapy and Oncology 114, 345-350 (2015).

40 S. Sone, S. Takashima, F. Li, Z. Yang, T. Honda, Y. Maruyama, M. Hasegawa, T. Yamanda, K. Kubo, K. Hanamura, and K. Asakura, "Mass screening for lung cancer with mobile spiral computed tomography scanner," The Lancet 351, 1242-1245 (1998).

41 T. Nawa, T. Nakagawa, S. Kusano, Y. Kawasaki, Y. Sugawara, and H. Nakata, "Lung Cancer Screening Using Low-Dose Spiral CT: Results of Baseline and 1-Year Follow-up Studies," Chest 122, 15-20 (2002).

42 S. Chong, K. S. Lee, M. J. Chung, T. S. Kim, H. Kim, O. J. Kwon, Y.-H. Choi, and C. H. Rhee, "Lung Cancer Screening with Low-Dose Helical CT in Korea: Experiences at the Samsung Medical Center," J Korean Med Sci 20, 402-408 (2005).

43 H. Ashraf, P. Tønnesen, J. Holst Pedersen, A. Dirksen, H. Thorsen, and M. Døssing, "Effect of CT screening on smoking habits at 1-year follow-up in the Danish Lung Cancer Screening Trial (DLCST)," Thorax 64, 388-392 (2009).

44 A. Lopes Pegna, G. Picozzi, M. Mascalchi, F. Maria Carozzi, L. Carrozzi, C. Comin, C. Spinelli, F. Falaschi, M. Grazzini, F. Innocenti, C. Ronchi, and E. Paci, "Design, recruitment and baseline results of the ITALUNG trial for lung cancer screening with low-dose CT," Lung Cancer 64, 34-40 (2009).

45 J. H. Pedersen, H. Ashraf, A. Dirksen, K. Bach, H. Hansen, P. Toennesen, H. Thorsen, J. Brodersen, B. G. Skov, M. Døssing, J. Mortensen, K. Richter, P. Clementsen, and N. Seersholm, "The Danish Randomized Lung Cancer CT Screening Trial-Overall Design and Results of the Prevalence Round," Journal of Thoracic Oncology 4, 608-614 (2009).

46 J. Gohagan, P. Marcus, R. Fagerstrom, P. Pinsky, B. Kramer, and P. Prorok, "Baseline Findings of a Randomized Feasibility Trial of Lung Cancer Screening With Spiral CT Scan vs Chest Radiograph: The Lung Screening Study of the National Cancer Institute," Chest 126, 114-121 (2004).

47 N. L. S. T. R. Team, "The National Lung Screening Trial: Overview and Study Design," Radiology 258, 243253 (2011).

48 T. Kubota, A. K. Jerebko, M. Dewan, M. Salganicoff, and A. Krishnan, "Segmentation of pulmonary nodules of various densities with morphological approaches and convexity models," Medical Image Analysis 15, 133-154 (2011).

49 J. Zhou, S. Chang, D. N. Metaxas, B. Zhao, M. S. Ginsberg, and L. H. Schwartz, in "An Automatic Method for Ground Glass Opacity Nodule Detection and Segmentation from CT Studies, 2006, p. 3062-3065.

50 Y. Zhu, Y. Tan, Y. Hua, G. Zhang, and J. Zhang, "Automatic Segmentation of Ground-Glass Opacities in Lung CT Images by Using Markov Random Field-Based Algorithms," J Digit Imaging 25, 409-422 (2012).

51 Y. Tan, L. H. Schwartz, and B. Zhao, "Segmentation of lung lesions on CT scans using watershed, active contours, and Markov random field," Medical Physics 40, 043502 (2013).

52 R. Tachibana and S. Kido, in "Automatic segmentation of pulmonary nodules on CT images by use of NCI lung image database consortium, 2006, p. 61440M-61440M-9. 


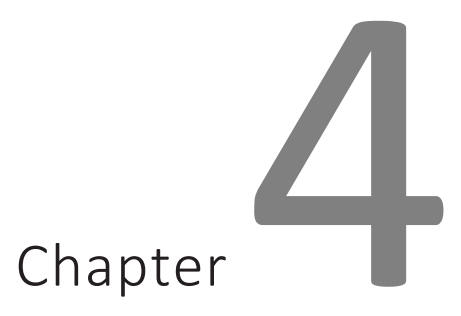

\section{Robust radiomics feature quantification using semiautomatic volumetric segmentation}

Published in: PLoS ONE 9(7); 2014

Robust radiomics feature quantification using semiautomatic volumetric segmentation Chintan Parmar*, Emmanuel Rios Velazquez*, Ralph Leijenaar, Mohammed Jermoumi, Sara Carvalho, Raymond H. Mak, Sushmita Mitra, B. Uma Shankar, Ron Kikinis, Benjamin Haibe-Kains, Philippe Lambin, Hugo J.W.L. Aerts

*These authors contributed equally to this work 


\section{ABSTRACT}

\section{Purpose}

Due to advances in the acquisition and analysis of medical imaging, it is currently possible to quantify the tumor phenotype. The emerging field of Radiomics addresses this issue by converting medical images into minable data by extracting a large number of quantitative imaging features. One of the main challenges of Radiomics is tumor segmentation. Where manual delineation is time consuming and prone to inter-observer variability, it has been shown that semi-automated approaches are fast and reduce inter-observer variability. In this study, a semiautomatic region growing volumetric segmentation algorithm, implemented in the free and publicly available 3D-Slicer platform, was investigated in terms of its robustness for quantitative imaging feature extraction.

\section{Materials and methods}

Fifty-six 3D-Radiomics features, quantifying phenotypic differences based on the tumor intensity, shape and texture, were extracted from the computed tomography images of twenty lung cancer patients. These Radiomics features were derived from the 3D-tumor volumes defined by three independent observers twice using 3D-Slicer, and compared to manual slice-by-slice delineations of five independent physicians in terms of intraclass correlation coefficient (ICC) and feature range.

\section{Results}

Radiomics features extracted from 3D-Slicer segmentations had significantly higher reproducibility ( $\mathrm{ICC}=0.85 \pm 0.15, \mathrm{p}=0.0009)$ compared to the features extracted from the manual segmentations $(I C C=0.77 \pm 0.17)$. Furthermore, we found that features extracted from 3D-Slicer segmentations were more robust, as the range was significantly smaller across observers $\left(p=3.819^{\mathrm{e}-07}\right)$, and overlapping with the feature ranges extracted from manual contouring (boundary lower: $p=0.007$, higher: $p=5.863^{\mathrm{e}-06}$ ).

\section{Conclusions}

Our results show that 3D-Slicer segmented tumor volumes provide a better alternative to the manual delineation process, as they are more robust for quantitative image feature extraction. Therefore, 3D-Slicer can be employed for quantitative image feature extraction and image data mining research in large patient cohorts. 


\section{INTRODUCTION}

Lung cancer affects approximately 1.6 million people worldwide every year [1]. The majority of lung cancer cases are non-small cell lung cancer (NSCLC), which has substantially poor prognosis and low survival rates [2].

Medical imaging is one of the major disciplines involved in oncologic science and treatment. By assessing human tissues non-invasively, imaging is extensively used for the detection, diagnosis, staging, and management of lung cancer. Due to the emergence of personalized medicine and targeted treatment, the requirement of quantitative image analysis has risen along with the increasing availability of medical data. Radiomics addresses this issue, and refers to the high throughput extraction of a large number of quantitative and mineable imaging features, assuming that these features convey prognostic and predictive information $[3,4]$. It focuses on optimizing quantitative imaging feature extraction through computational approaches and developing decision support systems, to accurately estimate patient risk and improve individualized treatment selection and monitoring.

Quantitative imaging features, extracted from medical images, are being extensively examined in clinical research. Several studies have shown the importance of imaging features for treatment monitoring and outcome prediction in lung and other cancer types [5-7]. For example, Ganeshan et al. assessed tumor heterogeneity in terms of imaging features extracted from routine computed tomography (CT) imaging in NSCLC, and reported their association with tumor stage, metabolism [8], hypoxia, angiogenesis [9] and patient survival [10]. Furthermore, several studies have uncovered the underlying correlation between gene expression profiles and radiographic imaging phenotype $[11,12]$. This kind of radiogenomic analysis has raised the utility of medical image descriptors in clinical oncology by projecting them as potential predictive biomarkers $[13,14]$.

To ensure the reliability of quantitative imaging features, accurate and robust tumor delineation is essential. Tumor segmentation is one of the main challenges of Radiomics, as manual delineation is prone to high inter-observer variability and represents a time-consuming task $[3,4]$. This makes the requirement of (semi)automatic and efficient segmentation methods evident. It has been shown that semiautomatic tumor delineation methods are better alternatives to manual delineations $[15,16]$. Recently, we have shown that for NSCLC, semiautomatic segmentation using 3D-Slicer (a free open source software platform for biomedical imaging research) reduces inter-observer variability and delineation uncertainty, compared to manual segmentation [17]. During the evaluation of quantitative imaging features as prognostic or predictive factors, it is essential to determine their variability with respect to the tumor delineation process. We hypothesize that quantitative imaging features extracted from semi-automatically segmented tumors have lower variability and are more robust compared to features extracted from manual tumor delineations. 
In this study we analyzed the robustness of imaging features derived from semiautomatically and manually segmented primary NSCLC tumors in twenty patients. We extracted fifty-six CT 3D-Radiomics features from 3D-Slicer segmentations made by three independent observers, twice, and compared them to the features extracted from manual delineations provided by five independent physicians. As 3D-Slicer is publicly available and easily accessible by download, it can have a large application in Radiomics to extract robust quantitative image features, and be employed for highthroughput data mining research of medical imaging in clinical oncology.

\section{MATERIAL AND METHODS}

\section{CT-PET scans of NSCLC patients}

The imaging data was acquired at MAASTRO Clinic in The Netherlands, as reported previously by Baardwijk et al [25]. In short, twenty patients with histologically verified non-small cell lung cancer, stage IB-IIIB, were included in this study. All patients received a diagnostic whole body positron emission tomography (PET)-computed tomography (CT) scan (Biograph, SOMATOM Sensation 16 with an ECAT ACCEL PET scanner; Siemens, Erlangen, Germany). Patients were instructed to fast at least six hours before administration of ${ }^{18}$ F-fluoro-2-deoxy-glucose (FDG) (MDS Nordion, Liège, Belgium), followed by physiologic saline $(10 \mathrm{~mL})$. After the injection of FDG, the patients were encouraged to rest for a period of 45 minutes. Next, free-breathing PET and CT images were acquired. The whole thorax spiral CT scan was acquired with intravenous contrast. The PET images were obtained in 5-min bed positions. The complete data set was then reconstructed iteratively with a reconstruction increment of $5 \mathrm{~mm}$. This study was approved by the local Medical Ethics Committee (Maastricht University Medical Center) and according to the Dutch law. As it was a retrospective study the requirement for informed consent was waived.

\section{Semiautomatic segmentation in 3D Slicer}

For the semiautomatic segmentation, the GrowCut algorithm implemented in 3D-Slicer was used (www.slicer.org). GrowCut is an interactive region growing segmentation strategy. Given an initial set of label points the algorithm automatically segments the remaining image by using cellular automation. The algorithm uses a competitive region growing approach and is considered to provide good accuracy and speed for both the 2D and 3D image segmentation. For $\mathrm{N}$-class segmentation the algorithm needs $\mathrm{N}$ initial sets of labeled pixels (one set corresponding to each class) from the user. Based on these, the algorithm automatically generates the region of interest (ROI), which is the convex hull of the user-labeled pixels with an additional margin. Next, it iteratively labels all the remaining pixels in the ROI using the user-given pixel labels. Pixel labeling is 
done using a weighted similarity score, which is a function of the neighboring pixel weights. An unlabeled pixel is labeled corresponding to the neighboring pixels that have the highest weights. The algorithm converges when all the pixels in the ROI have unchanged labels across several iterations.

3D-Slicer provides a graphical user interface (GUI) as the frontend and an efficient algorithm as the backend for the GrowCut segmentation. After loading the patient data, the process begins with the initialization of the foreground and background by marking the area inside and outside the tumor region. Next, the Growcut automatic competing region-growing algorithm gets activated, and segments the ROI into foreground and background regions. Thereafter, background and the surrounding isolated foreground pixels are removed following visual inspection.

\section{Manual tumor delineations}

Five physicians manually delineated the gross tumor volume (GTV) of the primary tumor based on fused PET-CT images using standard delineation protocol [which includes fixed window-level settings of both CT (lung W 1,700; L -300, mediastinum W 600; L 40) and PET scan (W 30,000; L 15,000) 2,7,22]. Radiation oncologists were mutually blind of each other's delineations. The primary GTV was defined for each patient based on combined CT and PET information along the axial plane. The physicians were given transversal, coronal, sagittal and 3D views simultaneously. A treatment planning system (XiO; Computer Medical System, Inc., St. Louis, MO) was used for performing delineations.

\section{Image processing and feature extraction}

All image data were loaded and analyzed in Matlab R2012b (The Mathworks, Natick, MA) using an adapted version of CERR (Computational Environment for Radiotherapy Research)[26], extended with in-house developed Radiomics image analysis software to extract imaging features.

From the five manual and the six 3D-Slicer segmentations, we extracted fifty-six 3DRadiomics features for the computed tomography scans. See figure 1 for an illustration of the employed methodology. A mathematical description of all features is shown in Supplement I. The radiomics features were divided in three groups: (I) tumor intensity, (II) shape, and (III) texture. The tumor intensity features consisted of features describing histogram of voxel intensity values contained within the volume of interest (VOI). Geometric features were calculated, describing the three-dimensional shape and size of the lesions. Textural features describing patterns or spatial distribution of voxel intensities, were calculated from gray level co-occurrence (GLCM) [27] and gray level run-length (GLRLM) matrices respectively [28]. Determining texture matrix representations requires the voxel intensity values within the VOI to be discretized. This step not only reduces image noise, but also normalizes intensities across all patients, allowing for a direct comparison of all calculated textural features between patients. Texture matrices 
were determined considering 26-connected voxels (i.e. voxels were considered to be neighbors in all 13 directions in three dimensions), and a distance of one voxel between consecutive voxels was set for computing co-occurrence and gray level run-length matrices. Features derived from co-occurrence and gray level run-length matrices were calculated by averaging their value over all 13 considered directions in three dimensions. Overall, the extracted imaging features comprised 15 features describing tumor intensity, 8 shape features and 33 textural features.

\section{Statistical analysis}

Intra-class correlation coefficient (ICC) was calculated in order to quantify the feature reproducibility. The ICC is a statistical measure, ranging between 0 and 1 , indicating null and perfect reproducibility, respectively. In order to determine the ICC for interobserver segmentations, variance estimates were obtained from two-way mixed effect model of analysis of variance (ANOVA). McGraw and Wong [29] defined ICC in case 3A to measure the absolute agreement as,

$$
I C C=\frac{M S_{R}-M S_{E}}{M S_{R}+(k-1) M S_{E}+\frac{k}{n}\left(M S_{C}-M S_{E}\right)}
$$

ICC values for intra-observer segmentations were obtained from one-way analysis of variance (ANOVA). It is defined using case 1 of McGraw and Wong [29] as,

$$
I C C=\frac{M S_{R}-M S_{W}}{M S_{R}+(k-1) M S_{W}}
$$

Where $M S_{R}=$ mean square for rows, $M S_{W}=$ mean square for residual sources of variance, $M S_{E}=$ mean square error, $M S_{C}=$ mean square for columns, $k=$ number of observers involved and $n=$ number of subjects. R package IRR (inter rater reliability) was used for ICC computation [30].

Wilcoxon rank-sum test was used to compare the reproducibility of image features derived from manual and 3D-Slicer segmentations methods. Two methods were considered to be significantly different when the $p$-value was lower than 0.05 . All data are expressed as mean \pm SD. All the analyses were performed in Matlab (The MathWorks Inc., Natick, MA, USA) and R (R Foundation for Statistical Computing, Vienna, Austria).

\section{RESULTS}

In order to assess the robustness of 3D-Slicer segmentation on CT imaging for quantitative image feature extraction, we assessed fifty-six 3D-radiomics features quantifying I) tumor intensity, II) tumor shape, and III) tumor texture (Fig. 1, Supplement I online). From twenty lung cancer patients we extracted the radiomics features from 3Dvolumes defined by three independent observers twice using 3D-Slicer, and compared them to manual delineations by five independent radiation oncologists. 
Since two 3D-Slicer segmentations from each of the three observers were considered for the analysis, the six 3D-Slicer segmentations were divided in to two sets, each having three segmentations (one from each observer). We calculated the intra-class correlation coefficient (ICC) for the radiomics features extracted from these two sets of three 3D-Slicer segmentations and five manual delineations. We observed that the radiomics features extracted from 3D-Slicer segmentations, had significantly higher reproducibility (avg. of two 3D-Slicer segmentation sets ICC $=0.85 \pm 0.15$ ) as compared to the features extracted from the manual segmentations (ICC $=0.77 \pm 0.17)(p=$ 0.0009 , Fig. 2).

Overall 38 out of the 56 features (68\%) showed higher ICC values for 3D-Slicer segmentations as compared to the manual ones. ICC values for all the assessed features are reported in Supplement II (online). To evaluate the robustness against multiple algorithmic initializations of the same observer, we computed ICC for the three intraobserver 3D-Slicer segmentation sets, each having two 3D-Slicer segmentations from the same observer. High ICC values (avg. of three intra-observer 3D-Slicer segmentation sets ICC $=0.90 \pm 0.17$ ) were observed for intra-observer segmentation groups. Fig. 3 depicts the ICC values corresponding to the inter-observer manual delineations and intra- \& inter-observer 3D-Slicer segmentations.

Intensity statistics and textural features showed significantly higher reproducibility (two sided Wilcoxon test $p=0.0006, p=0.009$, respectively) for 3D-Slicer based segmentations (avg. inter-observer ICC $=0.82 \pm 0.13, I C C=0.88 \pm 0.09$, respectively) as compared to manual delineations (ICC $=0.63 \pm 0.16$, ICC $=0.82 \pm 0.12$, respectively). No statistically significant difference (two sided Wilcoxon test $p=0.31$ ) was observed in ICC values for shape based features between the manual (ICC $=0.80 \pm 0.22$ ) and semiautomatic (avg. inter-observer ICC $=0.75 \pm 0.31$ ) groups. Fourteen out of 15 statistical features (93\%), and 20 out of 33 textural features (67\%), showed higher reproducibility (higher ICC) for 3D-Slicer segmentations as compared to manual delineations. For shape based descriptors there was no clear winner between the two segmentation strategies as 4 out of 8 (50\%) features turned out having higher ICC for 3D-Slicer segmentations. 

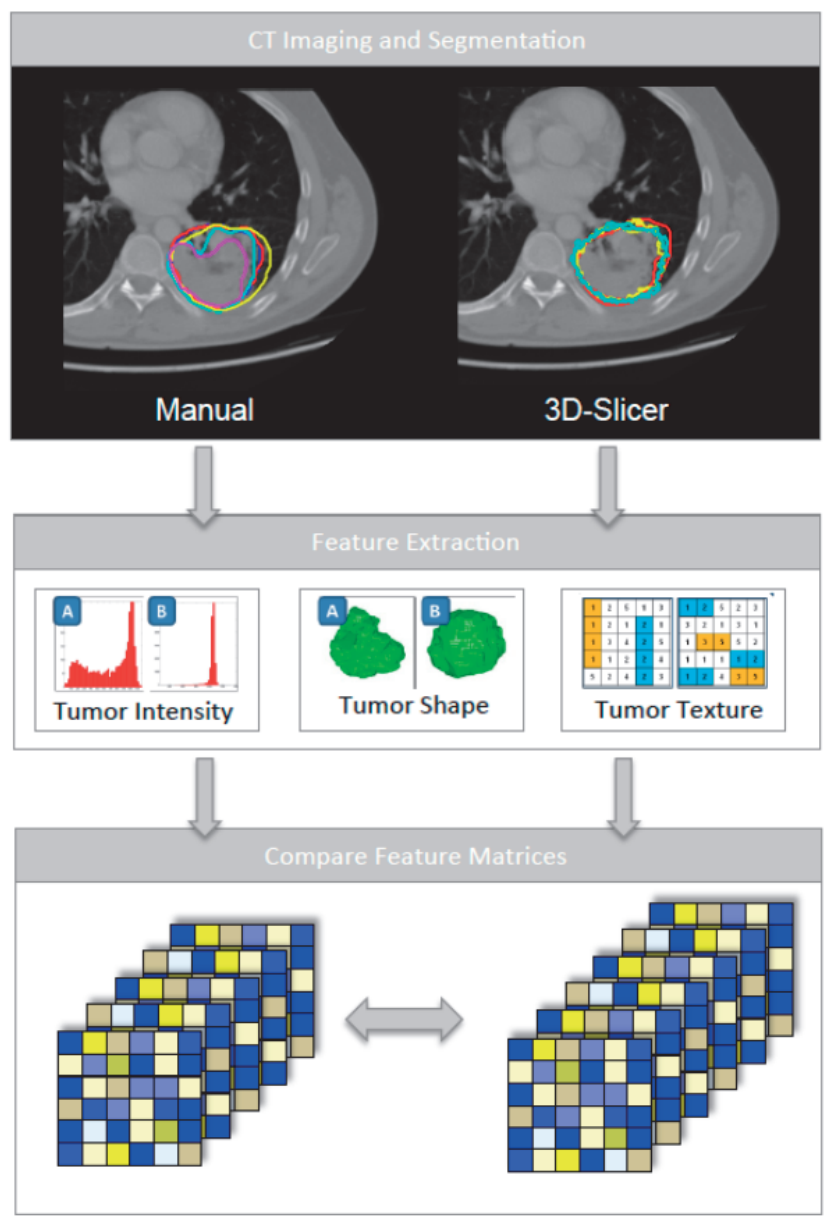

Figure 1. Schematic diagram depicting the overview of the analysis. A: First, we performed five manual delineations and six 3D-Slicer segmentations (three observers twice) on twenty lung tumors. B: Second, fiftysix radiomics features quantifying tumor intensity, texture and shape were extracted from these segmentations. C: Third, the resulting feature matrices were compared for robustness of the feature values.

We next classified the 56 features into three groups according to their ICC, as (I) having a high (ICC $\geq 0.8)$, (II) medium $(0.8>I C C \geq 0.5)$, or (III) low $(I C C<0.5)$ reproducibility (Supplement II online). For manual delineations, 52\% of all the assessed features had high, $45 \%$ had medium, and 3\% had low reproducibility on the other hand for 3D-Slicer based semiautomatic segmentations, 70\% features had high, 25\% had medium, and 5\% had low reproducibility. Therefore, reproducibility of the features was, in general, higher for 3D-Slicer segmentations. 
A

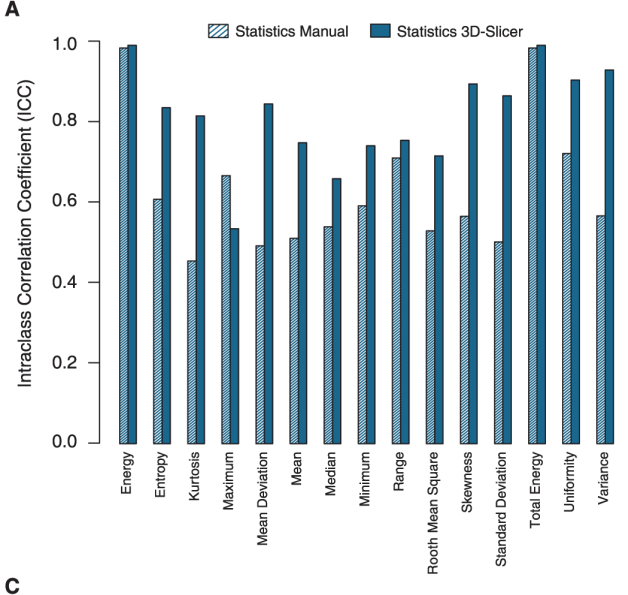

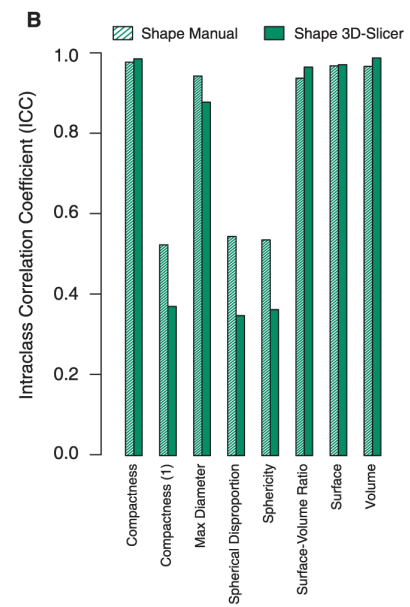

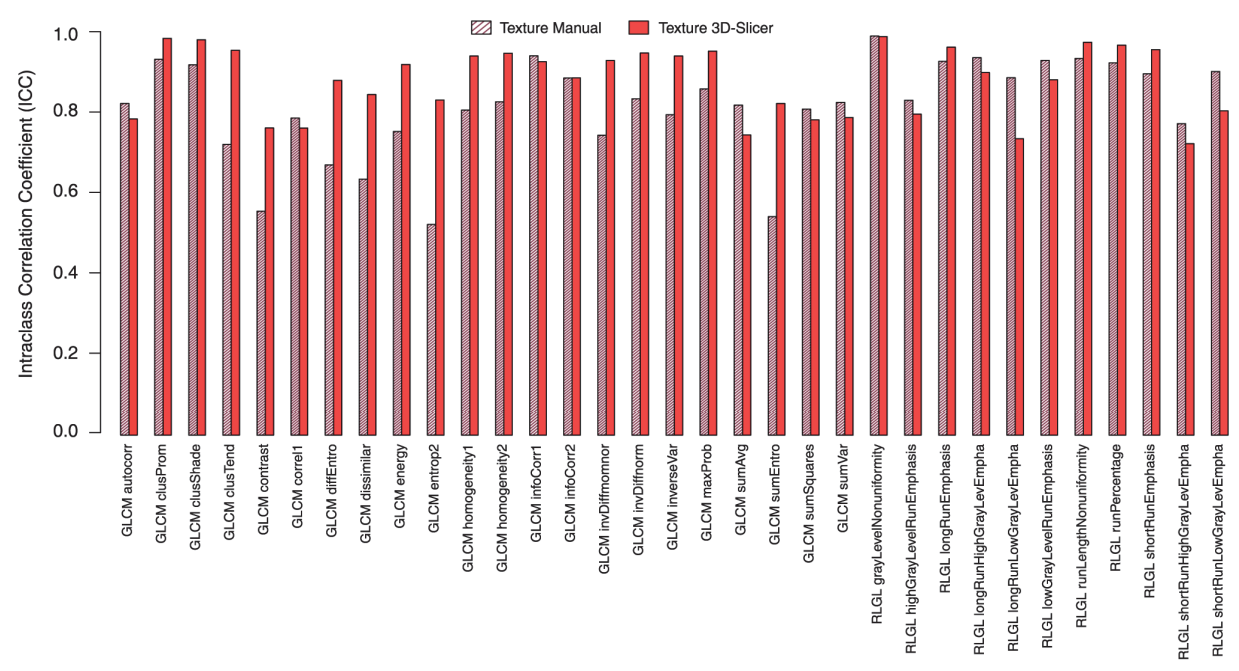

Figure 2 Feature wise comparison of Intra-class correlation coefficients (ICC) between manual and 3D-Slicer segmentations. A: First order statistics features. B: Shape based features. C Textural features.

Furthermore, it becomes important to determine whether the features extracted from semiautomatic segmentations capture the same tumor image properties as with manual delineations. Therefore, we compared the normalized range for all features between these two segmentation groups (Fig. 4). We normalized every feature value with respect to all 11 (5 manual + 6 3D-Slicer) segmentations, using Z-score normalization. We observed that the features extracted from 3D-Slicer based segmentations, spread over significantly smaller range across observers as compared to those of the manual delineations (two sided Wilcoxon test $p=3.819 \mathrm{e}-07$ ). Moreover, the features derived from 3D-Slicer segmentations overlapped in range with those of the manual delineations, as the lower(higher) limit(s) being significantly higher(lower) for the 3D-Slicer features (two sided Wilcoxon test $p=0.007, p=5.863 e-06$ ). This corroborates that the feature set, extracted from both the semiautomatic and manual strategies, correspond to simi- 
lar tumor image characteristics, with the features from 3D-Slicer having less variability across observers.

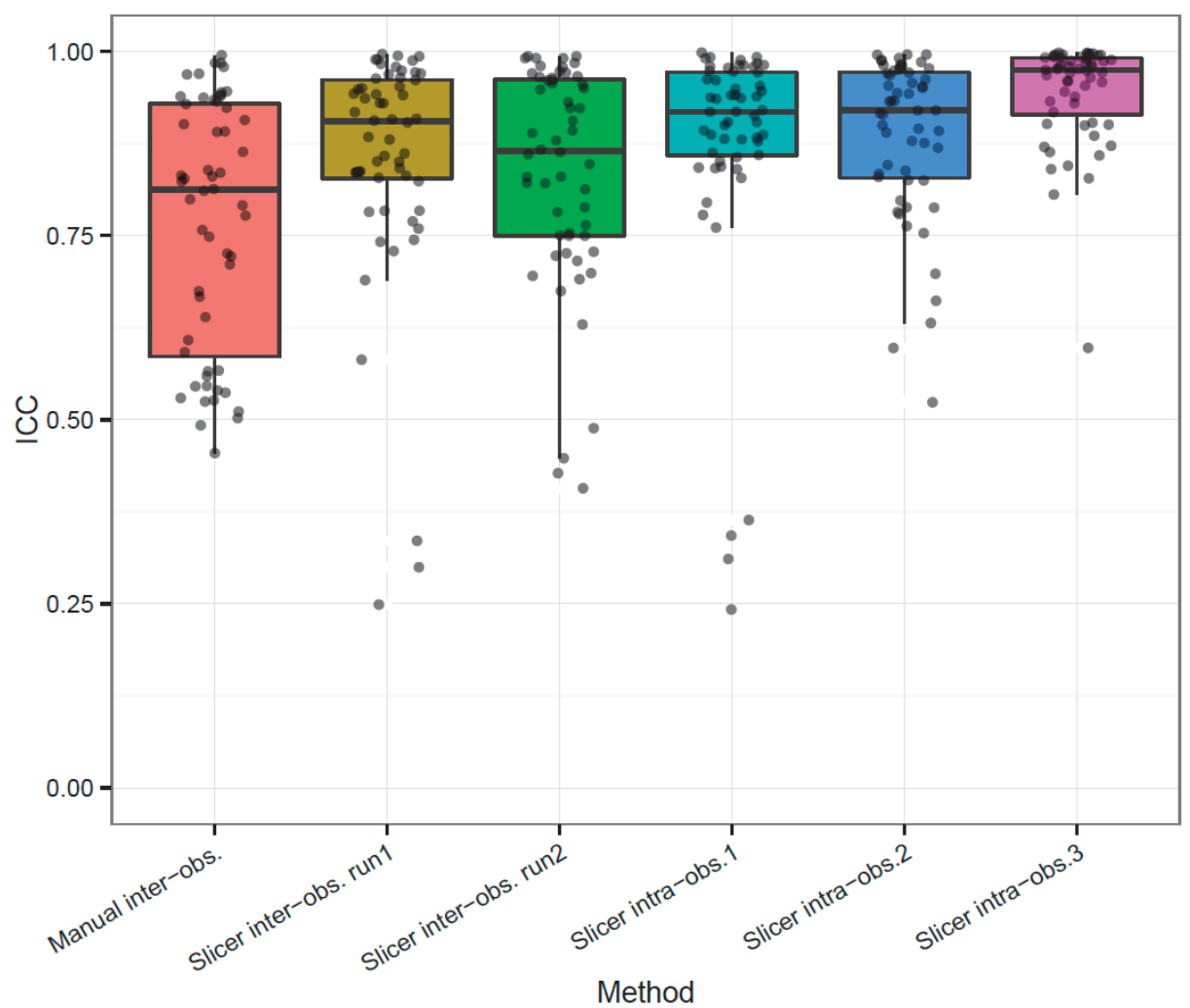

Figure 3 Box-plot comparing intra- and inter-observer reproducibility (ICC) of radiomics features. High interand intra- observer reproducibility (ICC) was observed for 3D-Slicer segmentations compared to the interobserver reproducibility (ICC) of manual delineations. From left the first box refers to the manual interobserver reproducibility (ICC), second and third boxes refer to the inter-observer reproducibility (ICC) of two different 3D-Slicer segmentation runs. Remaining three boxes refer to the intra-observer reproducibility (ICC) of 3D-Slicer segmentations.

\section{DISCUSSION}

Medical imaging is considered as one of the fundamental building blocks of clinical oncology. It is routinely used for cancer staging, treatment planning, and treatment response monitoring. Furthermore, recent developments in computational imaging, data mining and predictive analysis have broadened the scope of the imaging in clinical oncology. For example, quantitative imaging features extracted from CT images have been shown to predict $78 \%$ of the gene expression variability in hepatocellular carcinoma [11]. In a similar study, image descriptors, extracted from contrast enhanced MRI images of glioblastoma patients, predicted immunohistochemical identified protein 
expression patterns $[12,18]$. Recent computational approaches for image quantification, such as Radiomics, hypothesize that image descriptors extracted from tumor regions are associated with the risk of adverse events after treatment and could provide improved prognostic information for patient management $[3,4]$.

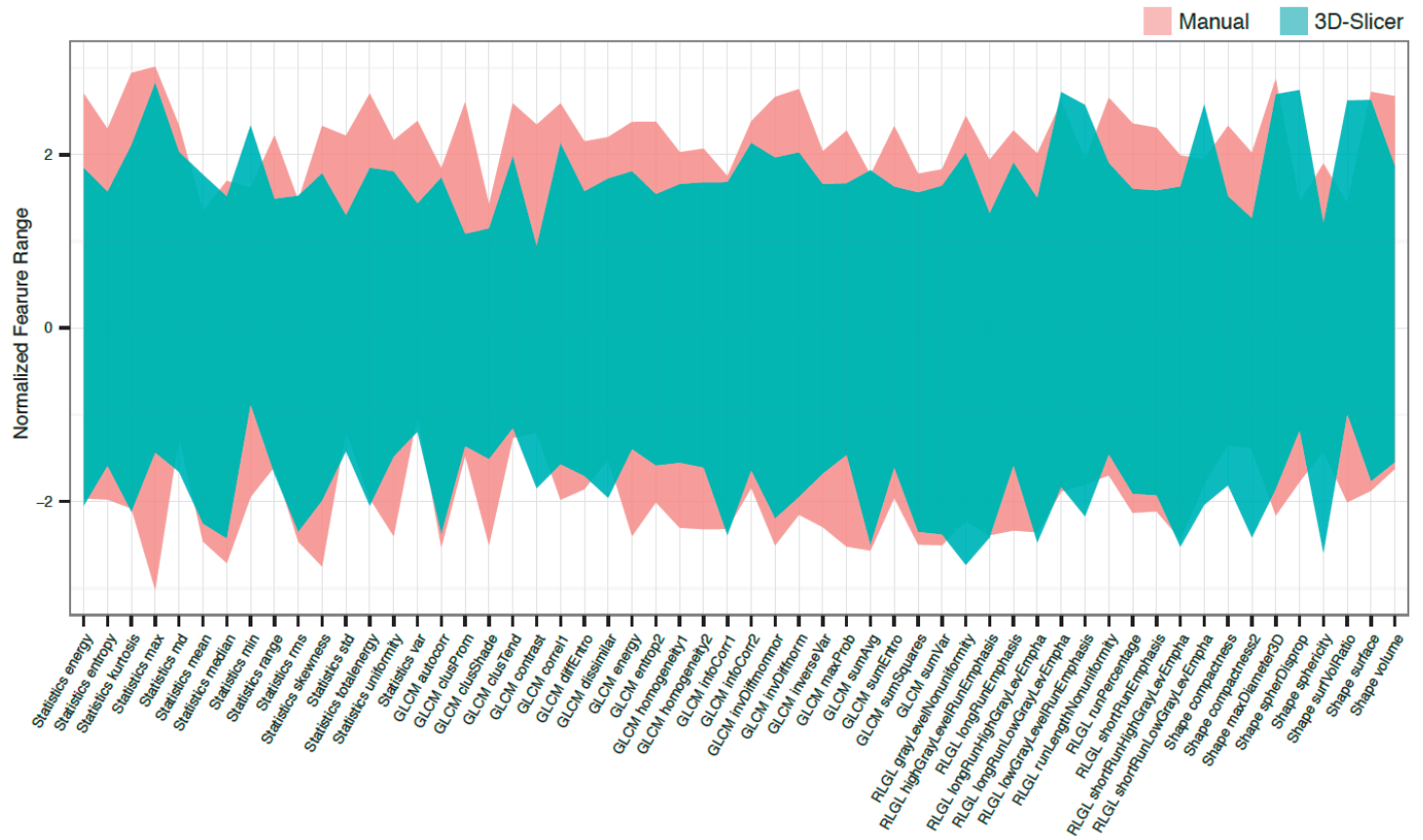

Figure 4 Comparison of normalized feature range between manual and 3D-Slicer segmentation groups. Radiomics features derived from 3D-Slicer segmentations had significantly smaller and overlapping range compared to that from manual delineations.

Accurate and efficient tumor segmentation is one the main challenges for the extraction of robust quantitative imaging features [4]. Manual segmentation suffers from high inter-observer variability and is time consuming [19]. It has been reported that semiautomatic segmentation strategies, as compared to manual delineation can improve tumor segmentation by reducing uncertainty as well as time $[15,17,19]$. These studies focused on tumor volumes while comparing semiautomatic and manual segmentation methods. However, tumor segmentation should also be evaluated in terms of the reliability of radiomics features derived from the volume of interest (VOI).

In this study, we investigated the robustness of quantitative imaging features, extracted from 3D-Slicer tumor segmentations, as compared to those, extracted from manual tumor delineations. Overall 3D-Slicer based semiautomatic segmentation method produced more reproducible radiomics features $(p=0.0009)$. We also analyzed different feature groups for their reproducibility, and observed that the difference in ICC, for intensity statistics and textural features, was statistically significant ( $p=0.0006$, $p=0.0094$, respectively) between the two segmentation strategies. The shape features, however did not significantly differ in reproducibility between the two strategies ( $p=$ 0.31). We also analyzed intra- and inter-observer reproducibility for 3D Slicer based 
semiautomatic segmentations. Three independent observers segmented each tumor twice, with different algorithmic initialization. Image descriptors demonstrated high intra-observer reproducibility for 3D-Slicer segmentations, which indicates their robustness over different seed point initializations. We also observed high inter-observer reproducibility in image descriptors for semiautomatic segmentations. Further reduction of inter-observer variability could be achieved by improving the semiautomatic segmentation strategy, i.e., by reducing observer interaction. Fully automatic methods requiring minimum user interaction, that may solve the complex problem of accurately defining the tumor boundaries, particularly in the case of large tumors with pleural attachment, are still a matter of investigation [20]. Although, current investigation shows that 3DSlicer segmentation provides a more robust alternative to manual contouring. Furthermore, as 3D-Slicer is publicly available and easily accessible by download, we expect its large utility in the field of quantitative imaging.

Recently the reproducibility of quantitative image features has been evaluated against repetitive test-retest CT image scans, acquired within fifteen minutes time interval, and was used to select the most informative radiomics features [4]. This work was expanded by Hunter et al, to evaluate the robustness of CT image features over three different imaging machines for identifying high quality multi-machine robust radiomics features [21]. In both these studies, since the NSCLC tumors were segmented by a single observer (by using a semiautomatic segmentation), the inter-observer reproducibility of the imaging features could not be evaluated. Leijenaar et al, have analyzed the stability to FDG-PET image features with respect to test-retest scans and interobserver delineations independently and reported a strong correlation between them [22]. Although they quantified the radiomics PET-based features for manual delineation stability, they did not compare it with that of semiautomatic tumor segmentations. No previous study, in our knowledge, has evaluated the reproducibility of quantitative CTbased imaging features in NSCLC, with respect to tumor segmentation methods.

One of the limitations of our study is not being able to associate these image descriptors with patient outcome due to cohort size and unavailability of clinical data. It would be interesting to investigate the effects of manual and semiautomatic segmentations on the image descriptor based prognostic performance. We hypothesize that more robust features having a stronger association with patient outcome are the most important biomarkers and play a vital role in high throughput data-mining research like Radiomics. Besides segmentation methods, other sources of variation should also be considered while evaluating quantitative image features. For instance, Galavis et al. investigated the variability in quantitative image descriptors due to different image acquisition modes and reconstruction parameters [23]. It has also been shown that different ways of image discretization influence the variability of textural features [24]. Although image acquisition, reconstruction and delineation protocols are typically standardized in the clinical practice, there still exists significant variation between imaging studies. Standardized protocols using semiautomatic segmentation tools are also 
warranted. Therefore, imaging features should be selected based on their robustness towards these sources of variation as well as their prognostic performance.

In conclusion, 3D-Slicer based semiautomatic segmentation significantly improves the robustness of radiomics feature quantification and thus could serve as a potential alternative to the time consuming manual segmentation process. 3D-Slicer can have a large application in radiomics research to extract robust quantitative image features, and be employed for high-throughput data mining research of medical imaging in clinical oncology.

\section{SUPPLEMENTARY INFORMATION}

http://journals.plos.org/plosone/article/file?type=supplementary\&id=info:doi/10.1371/j ournal.pone.0102107.s001

http://journals.plos.org/plosone/article/file?type=supplementary\&id=info:doi/10.1371/j ournal.pone.0102107.s002 


\section{REFERENCES}

1. Jemal A, Bray F, Center MM, Ferlay J, Ward E, et al. (2011) Global cancer statistics. CA: A Cancer Journal for Clinicians 61: 69-90.

2. van Baardwijk A, Wanders S, Boersma L, Borger J, Öllers M, et al. (2010) Mature results of an individualized radiation dose prescription study based on normal tissue constraints in stages I to III non-small-cell lung cancer. Journal of Clinical Oncology 28: 1380-1386.

3. Lambin P, Rios-Velazquez E, Leijenaar R, Carvalho S, van Stiphout RG, et al. (2012) Radiomics: Extracting more information from medical images using advanced feature analysis. European Journal of Cancer 48 : 441-446.

4. Kumar V, Gu Y, Basu S, Berglund A, Eschrich SA, et al. (2012) Radiomics: the process and the challenges. Magnetic Resonance Imaging 30: 1234-1248.

5. Vaidya M, Creach KM, Frye J, Dehdashti F, Bradley JD, et al. (2012) Combined PET/CT image characteristics for radiotherapy tumor response in lung cancer. Radiotherapy and Oncology 102: 239-245.

6. El Naqa I, Grigsby P, Apte A, Kidd E, Donnelly E, et al. (2009) Exploring feature-based approaches in PET images for predicting cancer treatment outcomes. Pattern Recognition 42: 1162-1171.

7. Tixier F, Le Rest CC, Hatt M, Albarghach N, Pradier O, et al. (2011) Intra-tumor heterogeneity on baseline 18 F-FDG PET images characterized by textural features predicts response to concomitant radiochemotherapy in esophageal cancer. Journal of Nuclear Medicine (JNM) 52: 369-378.

8. Ganeshan B, Abaleke S, Young RC, Chatwin CR, Miles KA (2010) Texture analysis of non-small cell lung cancer on unenhanced computed tomography: initial evidence for a relationship with tumour glucose metabolism and stage. Cancer Imaging 10: 137-143.

9. Ganeshan B, Goh V, Mandeville HC, Ng QS, Hoskin PJ, et al. (2013) Non-small cell lung cancer: histopathologic correlates for texture parameters at CT. Radiology 266: 326-336.

10. Ganeshan B, Panayiotou E, Burnand K, Dizdarevic S, Miles K (2012) Tumour heterogeneity in non-small cell lung carcinoma assessed by $\mathrm{CT}$ texture analysis: a potential marker of survival. European Radiology 22: 796-802.

11. Segal E, Sirlin CB, Ooi C, Adler AS, Gollub J, et al. (2007) Decoding global gene expression programs in liver cancer by noninvasive imaging. Nature Biotechnology 25: 675-680.

12. Zinn PO, Majadan B, Sathyan P, Singh SK, Majumder S, et al. (2011) Radiogenomic mapping of ede$\mathrm{ma} /$ cellular invasion MRI-phenotypes in glioblastoma multiforme. PLoS One 6: e25451.

13. Buckler AJ, Bresolin L, Dunnick NR, Sullivan DC (2011) Quantitative imaging test approval and biomarker qualification: interrelated but distinct activities. Radiology 259: 875-884.

14. Buckler AJ, Bresolin L, Dunnick NR, Sullivan DC (2011) A collaborative enterprise for multi-stakeholder participation in the advancement of quantitative imaging. Radiology 258: 906-914.

15. Rios Velazquez E, Aerts HJ, Gu Y, Goldgof DB, De Ruysscher D, et al. (2012) A semiautomatic CT-based ensemble segmentation of lung tumors: Comparison with oncologists' delineations and with the surgical specimen. Radiotherapy and Oncology 105: 167-173.

16. Heye T, Merkle EM, Reiner CS, Davenport MS, Horvath JJ, et al. (2013) Reproducibility of Dynamic Contrast-enhanced MR Imaging. Part II. Comparison of Intra-and Interobserver Variability with Manual Region of Interest Placement versus Semiautomatic Lesion Segmentation and Histogram Analysis. Radiology 266: 812-821.

17. Rios Velazquez E, Parmar C, Jermoumi M, Mak RH, van Baardwijk A, et al. (2013) Volumetric CT-based segmentation of NSCLC using 3D-Slicer. Scientific Reports 3: DOI: 10.1038/srep03529.

18. Zinn PO, Sathyan P, Mahajan B, Bruyere J, Hegi M, et al. (2012) A novel volume-age-KPS (VAK) glioblastoma classification identifies a prognostic cognate microRNA-gene signature. PLoS One 7: e41522.

19. Egger J, Kapur T, Fedorov A, Pieper S, Miller JV, et al. (2013) GBM Volumetry using the 3D Slicer Medical Image Computing Platform. Scientific Reports 3.

20. Gu Y, Kumar V, Hall LO, Goldgof DB, Li C-Y, et al. (2012) Automated delineation of lung tumors from CT images using a single click ensemble segmentation approach. Pattern Recognition 46: 692-702. 
21. Hunter LA, Krafft S, Stingo F, Choi H, Martel MK, et al. (2013) High quality machine-robust image features: Identification in nonsmall cell lung cancer computed tomography images. Medical Physics 40: DOI:10.1118/1111.4829514.

22. Leijenaar RT, Carvalho S, Velazquez ER, Van Elmpt WJ, Parmar C, et al. (2013) Stability of FDG-PET Radiomics features: An integrated analysis of test-retest and inter-observer variability. Acta Oncologica 52: 1391-1397.

23. Galavis PE, Hollensen C, Jallow N, Paliwal B, Jeraj R (2010) Variability of textural features in FDG PET images due to different acquisition modes and reconstruction parameters. Acta Oncologica 49: 10121016.

24. Tixier F, Hatt M, Le Rest CC, Le Pogam A, Corcos L, et al. (2012) Reproducibility of tumor uptake heterogeneity characterization through textural feature analysis in 18F-FDG PET. Journal of Nuclear Medicine 53: 693-700.

25. Van Baardwijk A, Bosmans G, Boersma L, Buijsen J, Wanders S, et al. (2007) Pet-ct-based autocontouring in non-small-cell lung cancer correlates with pathology and reduces interobserver variability in the delineation of the primary tumor and involved nodal volumes. International Journal of Radiation Oncology* Biology* Physics 68: 771-778.

26. Deasy JO, Blanco Al, Clark VH (2003) CERR: a computational environment for radiotherapy research. Medical Physics 30: 979-985.

27. Haralick RM, Shanmugam K, Dinstein IH (1973) Textural features for image classification. IEEE Transactions on Systems, Man and Cybernetics SMC-3: 610-621.

28. Galloway MM (1975) Texture analysis using gray level run lengths. Computer Graphics and Image Processing 4: 172-179.

29. McGraw KO, Wong S (1996) Forming inferences about some intraclass correlation coefficients. Psychological methods 1: 30-46.

30. Gamer M, Lemon J, Fellows I, Singh P (2013) IRR: Various coefficients of interrater reliability and agreement. R package version 0.84. CRAN: http://www.r-project.org.13. Chen AY, Halpern M (2007) Factors predictive of survival in advanced laryngeal cancer. Arch Otolaryngol Head Neck Surg 133:12701276 

PART

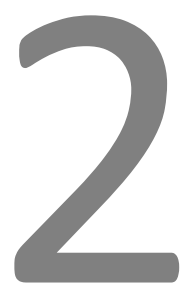

Machine learning methods for Radiomics 



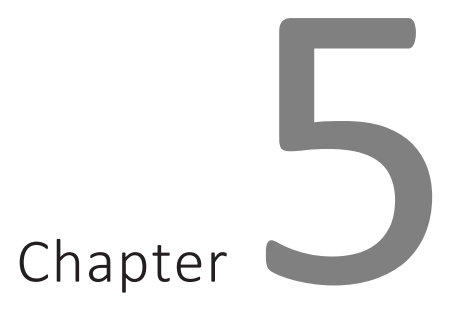

\section{Decoding tumour phenotype by noninvasive imaging using a quantitative radiomics approach}

Published in: Nature Communications. 5:4006; 2014.

Decoding tumour phenotype by noninvasive imaging using a quantitative radiomics approach

Hugo J.W.L. Aerts*, Emmanuel Rios Velazquez*, Ralph T.H. Leijenaar, Chintan Parmar, Patrick Grossmann, Sara Cavalho, Johan Bussink, René Monshouwer, Benjamin HaibeKains, Derek Rietveld, Frank Hoebers, Michelle M. Rietbergen, C. René Leemans, Joseph O. Deasy, Andre Dekker, John Quackenbush, Robert J. Gillies, Philippe Lambin *These authors contributed equally to this work 


\section{ABSTRACT}

Human cancers exhibit strong phenotypic differences that can be visualized noninvasively by medical imaging. Radiomics refers to the comprehensive quantification of tumour phenotypes by applying a large number of quantitative image features. Here we present a radiomic analysis of 440 features quantifying tumour image intensity, shape and texture, which are extracted from computed tomography data of 1,019 patients with lung or head-and-neck cancer. We find that a large number of radiomic features have prognostic power in independent data sets of lung and head-and-neck cancer patients, many of which were not identified as significant before. Radiogenomics analysis reveals that a prognostic radiomic signature, capturing intratumour heterogeneity, is associated with underlying gene-expression patterns. These data suggest that radiomics identifies a general prognostic phenotype existing in both lung and head-and-neck cancer. This may have a clinical impact as imaging is routinely used in clinical practice, providing an unprecedented opportunity to improve decision-support in cancer treatment at low cost. 


\section{INTRODUCTION}

Medical imaging is one of the major factors that have informed medical science and treatment. By assessing the characteristics of human tissue non-invasively, imaging is often used in clinical practice for oncologic diagnosis and treatment guidance ${ }^{1-3}$. A key goal of imaging is 'personalized medicine', were treatment is increasingly tailored based on specific characteristics of the patient and their disease ${ }^{4}$.

Much of the discussion of personalized medicine has focused on molecular characterization using genomic and proteomic technologies. However, as tumors are spatially and temporally heterogeneous, these techniques are limited. They require biopsies or invasive surgeries to extract and analyze what are generally small portions of tumor tissue, which do not allow for a complete characterization of the tumor. Imaging has great potential to guide therapy because it can provide a more comprehensive view of the entire tumor and it can be used on an on-going basis to monitor the development and progression of the disease or its response to therapy. Further, imaging is noninvasive and is already often repeated during treatment in routine practice, on the contrary of genomics or proteomics, which are still challenging to implement into clinical routine.

The most widely used imaging modality in oncology is $x$-ray computed tomography (CT), which assesses tissue density. Indeed, CT images of lung cancer tumors exhibit strong contrast reflecting differences in the intensity of a tumor on the image, intra tumor texture, and tumor shape (Fig.1a). However, in clinical practice, tumor response to therapy is only measured using 1 or 2 dimensional descriptors of tumor size (RECIST and $\mathrm{WHO}$, respectively) $)^{5}$. While a change in tumor size can indicate response to therapy, it often does not predict overall or progression free survival ${ }^{6,7}$. Although some investigations have characterized the appearance of a tumor on CT images, these characteristics are typically described subjectively and qualitative ("moderate heterogeneity", "highly spiculated", "large necrotic core"). However, recent advances in image acquisition, standardization, and image analysis, allow for objective and precise quantitative imaging descriptors that could potentially be used as non-invasive prognostic or predictive biomarkers.

Radiomics is an emerging field that converts imaging data into a high dimensional mineable feature space using a large number of automatically extracted datacharacterization algorithms ${ }^{8,9}$. We hypothesize that these imaging features capture distinct phenotypic differences of tumours and may have prognostic power and thus clinical significance across different diseases. Here we assess the clinical relevance of 440 radiomic features, many of which currently have no known clinical significance, in seven independent cohorts consisting of 1,019 lung cancer and head-and-neck cancer patients. Two data sets are used to assess the stability of the features, four data sets to assess the prognostic value of radiomic features on lung cancer patients and head-andneck cancer patients, and one data set for association with gene-expression profiles of lung cancer patients (Fig. 2). Our results reveal that radiomics data contain strong prog- 
nostic information in both lung and head-and-neck cancer patients, and are associated with the underlying gene-expression patterns. These results suggest that radiomics decodes a general prognostic phenotype existing in multiple cancer types. Radiomics can have a large clinical impact, as imaging is used in routine practice worldwide, providing a method that can quantify and monitor phenotypic changes during treatment.

a)

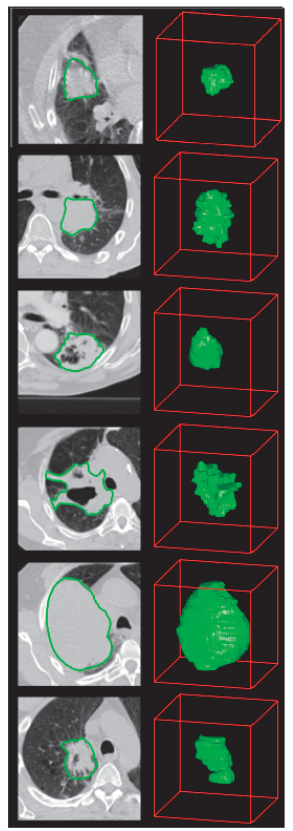

b)

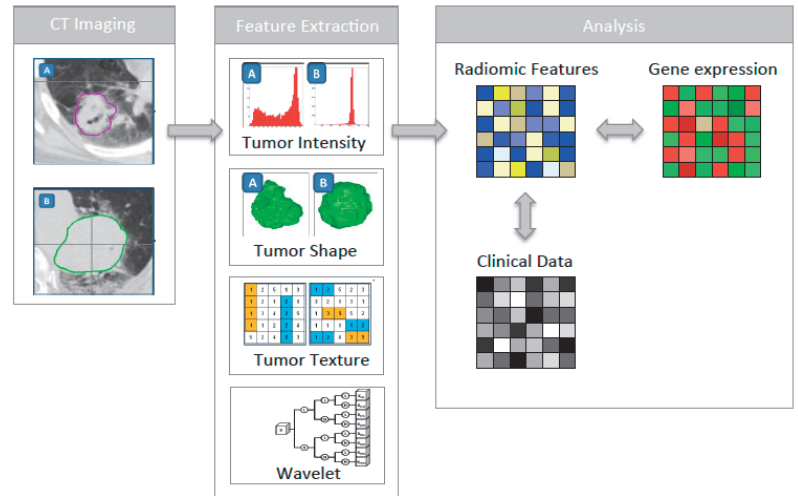

Figure 1Extracting Radiomics data from images. (a) Tumors are different. Example computed tomography (CT) images of lung cancer patients. (b) Strategy for extracting Radiomics data from images.

\section{RESULTS}

First, we defined 440 quantitative image features describing tumor phenotype characteristics by: I) tumor image intensity, II) shape, III) texture and IV) multi-scale Wavelet (Fig.1b, Supplement I online).

To investigate radiomic expression patterns we extracted radiomic features from the Lung1 dataset, consisting of 422 NSCLC cancer patients (Fig.2). Unsupervised clustering revealed clusters of patients with similar radiomic expression patterns (Fig.3). We compared the three main clusters of patients with clinical parameters (Fig.3b), and found significant association with primary tumor stage (T-stage; $p<1 \times 10^{-24}$ ) and overall stage $\left(p<1 \times 10^{-6}\right)$, wherein cluster I was associated with lower stages. N-stage (lymph node) and $M$-stage (metastasis), however, showed no correspondence with the radiomic expression patterns ( $p=0.27$, and $p=0.73$ respectively). 


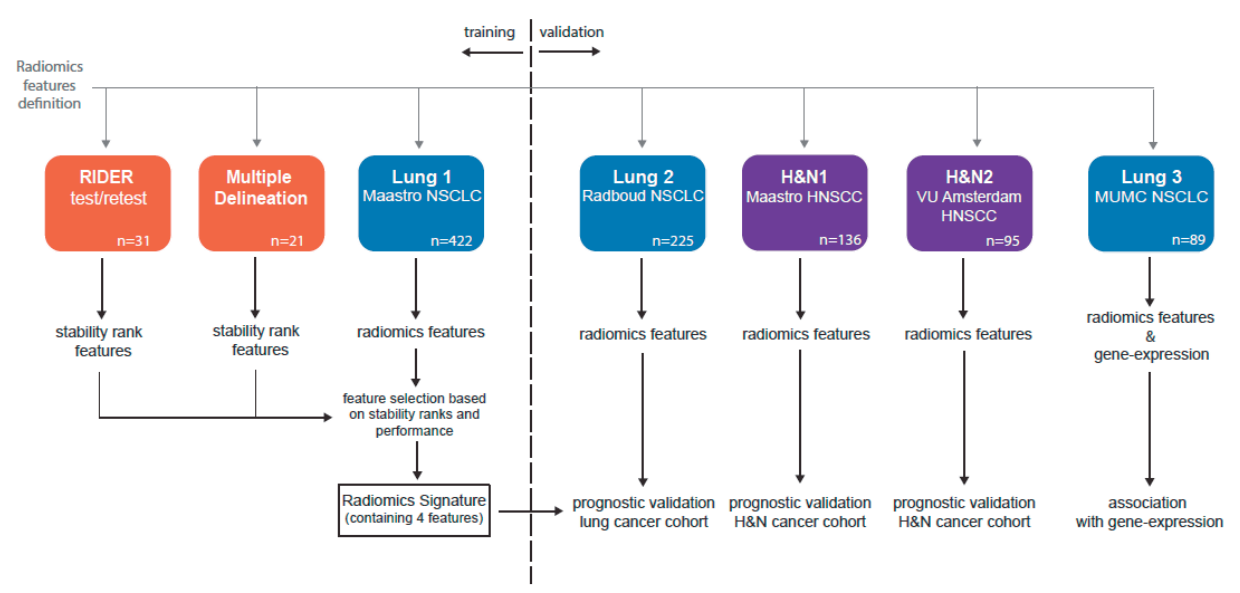

Figure 2 Analysis workflow. The defined radiomic features algorithms (more information Supplement I) were applied to seven different datasets (more information Supplement II). Two datasets were used to calculate the feature stability ranks, RIDER test/retest and Multiple Delineation respectively (both orange). The Lung1 dataset was used as training dataset. Lung2, H\&N1, and H\&N2 were used as validation datasets. The Lung3 dataset was used for association of the radiomic signature with gene expression profiles.

Furthermore, a significant association with histology ( $p=0.31 \times 10-3)$ was observed, wherein squamous cell carcinoma showed a higher presence in cluster II. Looking at the representation of the feature groups (Fig.3c), there was no correspondence between the feature group and radiomic expression patterns.

The analysis was divided in training and validation phases (Fig.2). For the training phase, we first explored feature stability determined in both test-retest and interobserver setting. Using the publicly available RIDER ${ }^{10}$ dataset, consisting of 31 sets of test-retest CT-scans that were acquired approximately 15 minutes apart, we tested how consistent the radiomic features were between the test and retest scan. The multiple delineation dataset, where five oncologists delineated lesions on CT scans from 21 patients $^{11}$, was used to test the stability of the radiomic features to variation in manual delineations.

For each feature we compared the stability ranks for test-retest and multiple delineation with prognosis in the Lung1 training dataset. Although the stability ranks did not use any information about prognosis, in general, features with higher stability for test retest and delineation inaccuracies showed higher prognostic performance (Extended Data Figure 1). This is possibly due to reduced amount of noise in the stable features and supports the use of stability ranks for feature selection.

The possible association of radiomic features with survival was then explored by Kaplan-Meier survival analysis. For training we used the Lung1 dataset, and for validation the Lung2, H\&N1, H\&N2 datasets (Fig.2). The radiomic features were not normalized on any dataset, and only the raw values were used that were directly computed from the DICOM images. 
a)

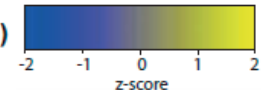

Patients

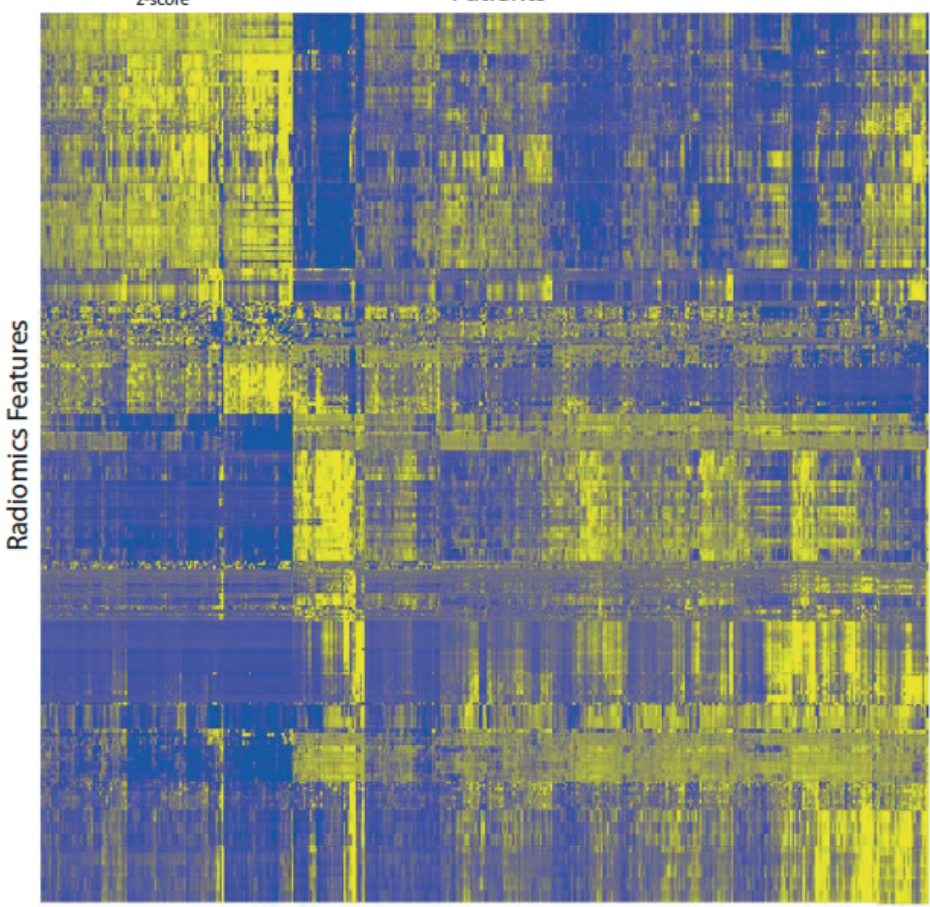

c) Clusters b)

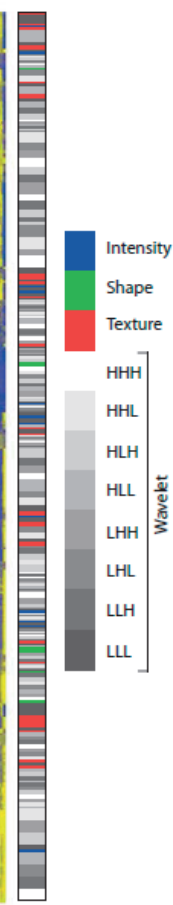

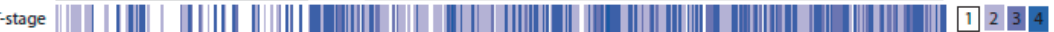

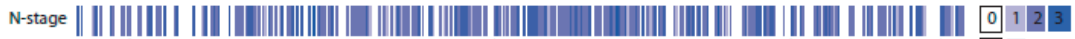

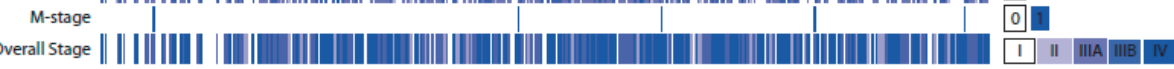

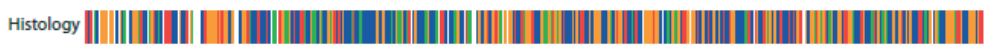

$\square$ adenocarcinoma $\square$ squamous cell carcinoma $\square$ large cell carcinoma $\square$ not otherwise specified (nos) $\square$ NA

Figure 3 Radiomics heat map. (a) Unsupervised clustering of lung cancer patients (Lung1 set, $n=422$ ) on the $y$ axis and radiomic feature expression $(n=440)$ on the $x$-axis, revealed clusters of patients with similar radiomic expression patterns. (b) Clinical patient parameters for showing significant association of the radiomic expression patterns with primary tumor stage (T-stage; $\left.p<1 \times 10^{-24}\right)$, overall stage $\left(p<1 \times 10^{-6}\right)$, and histology $\left(p=0.31 \times 10^{-3}\right)$. (c) Correspondence of radiomic feature groups with the clustered expression patterns.

To ensure a completely independent validation, the median value of each feature was computed on the training Lung1 dataset, and locked for use as a threshold in the validation datasets in order to assess the survival differences without retraining (Fig.2). In

Extended Data Figure 2 we show Kaplan-Meier survival curves for four representative features. Features describing heterogeneity in the primary tumor were associated with worse survival in all four datasets. Also, patients with more compact/spherical tumors had better survival probability.

Overall, the median threshold derived from Lung1 yielded a significant survival difference for 238 features (54\% of in total 440, FDR 10\%) in the Lung2 validation dataset. Furthermore, there was a significant survival difference for 135 features (31\%) in H\&N1 
and for 186 features in H\&N2 (42\%). Sixty-six (15\%) of the features derived from Lung1 were significant for survival in all three-validation datasets (Lung2, H\&N1, and H\&N2).

To test the multivariate performance of a radiomic signature, we used the workflow depicted in Extended Data Figure 3. We focused our analysis on the 100 most stable features, which were determined by averaging the stability ranks of RIDER dataset and Multiple Delineation dataset. To remove redundancy within the radiomic information, we select the single best performing radiomic feature from each of the four feature groups, and combined these top four features into a multivariate Cox proportional hazards regression model for prediction survival.

The resulting radiomic signature consisted of I) "Statistics Energy" (Supplement I Feature 1.1) describing the overall density of the tumor volume, II) "Shape Compactness" (Feature 2.2) quantifying how compact the tumor shape is, III) "Gray Level Nonuniformity" (Feature 3.25) a measure for intra-tumor heterogeneity, and IV) Wavelet "Gray Level Nonuniformity HLH" (Feature Group4), also describing intra-tumor heterogeneity after decomposing the image in mid-frequencies. The weights of each of the features in the signature were fitted on the training dataset Lung1. The performance of the four feature radiomic signature was validated in the datasets Lung2, H\&N1, and $\mathrm{H} \& N 2$ (Fig.4a) using the concordance index $(\mathrm{Cl})$, which is a generalization of the area under the ROC-curve ${ }^{12}$.

The radiomic signature had good performance on the Lung2 data $(\mathrm{Cl}=0.65, p=$ $\left.2.91 \times 10^{-09}\right)$, and a high performance in $\mathrm{H} \& N 1\left(\mathrm{Cl}=0.69, p=7.99 \times 10^{-07}\right)$ and $\mathrm{H} \& N 2$ $\left(\mathrm{Cl}=0.69, p=3.53 \times 10^{-06}\right)$. Although volume had a good performance in all datasets, the radiomic signature performed significantly better, suggesting that radiomic features contain relevant, complementary information for prognosis (Extended Data Table 1). Furthermore, combining the radiomic signature with volume was significantly better than volume alone in all datasets.

Comparing the radiomic signature to the TNM staging ${ }^{13}$, we see that the signature performance was better in both Lung2 and H\&N2 and comparable in H\&N1. Important$l y$, combining the radiomic signature with TNM staging showed a significant improvement in all datasets, compared with TNM staging alone. Furthermore, we assessed if the radiomics signature preserved the significant prognostic performance compared to the treatment patients received. We found that the signature preserved its prognostic performance for all treatment groups (radiation, or concurrent chemo-radiation), for both Lung and H\&N cancer patients (see Extended Data Table 2), demonstrating the complementary value of radiomics for each treatment type. 
a)

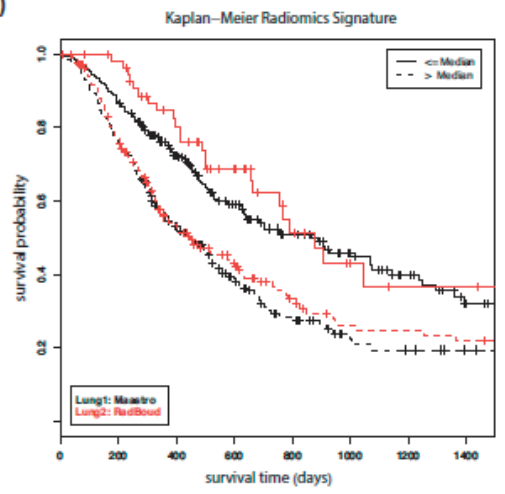

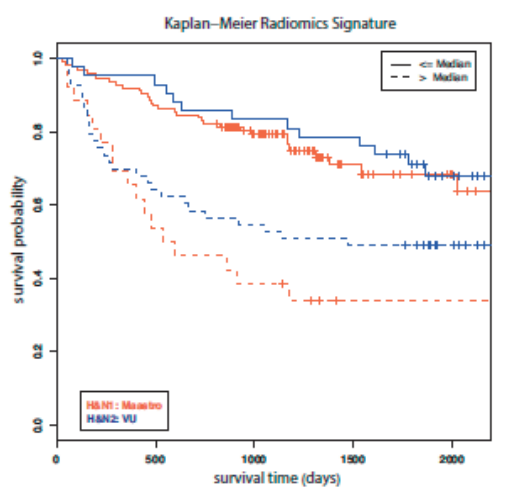

b)

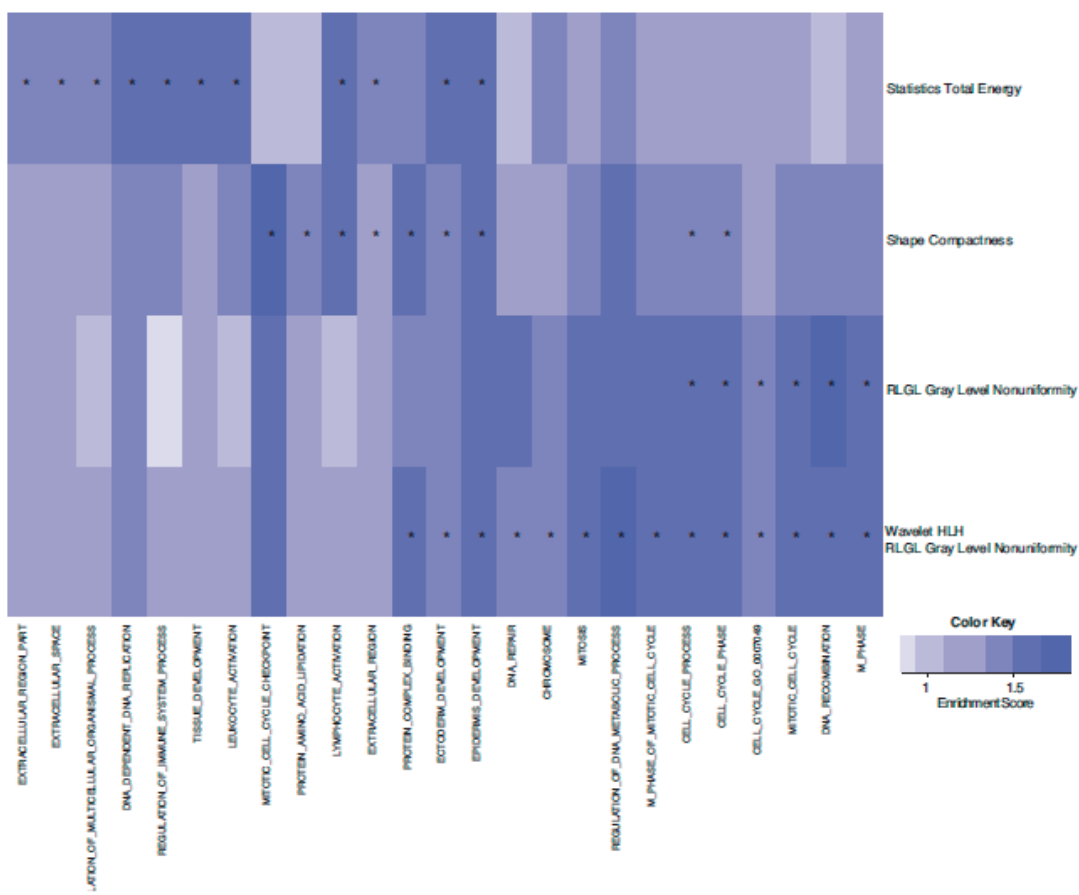

Figure 4 (a) Radiomic signature performance. Performance of the radiomic signature on the lung cancer datasets (left) and the head and neck cancer datasets (right). The signature was built on the Lung1 data $(n=422)$. The signature had good performance in the Lung $\left(\mathrm{Cl}=0.65, p=2.91 \times 10^{-09}, n=225\right)$, and a high performance in $\mathrm{H} \& \mathrm{~N} 1\left(\mathrm{Cl}=0.69, \mathrm{p}=7.99 \times 10^{-07}, \mathrm{n=136}\right)$ and $\mathrm{H} \& \mathrm{~N} 2\left(\mathrm{Cl}=0.69, \mathrm{p}=3.53 \times 10^{-06}, \mathrm{n}=95\right)$ validation datasets. (b) Association of radiomic signature features and gene expression using Gene Set Enrichment Analysis (GSEA). Gene sets that have been significantly enriched for at least one of the four-radiomic features are indicated with an asterisk. The corresponding normalized enrichment scores (NES), GSEA's primary statistic, for all radiomic signature features is displayed in a heat-map, where light blue means low, and dark blue means high NES.

Human papillomavirus (HPV) is an important determinant in head and neck cancer patients, especially those with oropharyngeal carcinoma for prognosis and may guide 
future treatment selection. We did not find a significant association between radiomic signature prediction and HPV status in a combined analysis in the H\&N1 and H\&N2 dataset ( $p=0.17$, Extended Data Table 2). However, we found that the signature preserved its prognostic performance in the HPV negative group $(\mathrm{Cl}=0.66)$, consisting of the majority of patients $(76 \%, n=130)$, demonstrating the complementary value of Radiomics to HPV screening.

To assess the association between the radiomic signature and the underlying biology, we compared the radiomic signature with gene-expression profiles (Lung3 dataset, Fig.2) using gene-set enrichments analysis (GSEA) $)^{1,14}$. We found significant associations between the signature features and gene-expression patterns (Fig.4b). Further, the radiomic features are significantly associated with different biologic gene-sets, demonstrating that radiomic features probe different biologic mechanisms. It is noteworthy that both intra-tumor heterogeneity features in the signature (Feature III and IV) were strongly correlated with cell cycling pathways, indicating an increased proliferation for more heterogeneous tumors.

\section{DISCUSSION}

Medical imaging is one of the major factors informing medical science and treatment. Its potential resides in its ability to assess the characteristics of human tissue noninvasively, and therefore is routinely used in clinical practice for oncologic diagnosis and treatment guidance and monitoring.

However, traditionally, medical imaging has been a subjective or qualitative science. Recent advances in medical imaging acquisition and analysis, allow the high-throughput extraction of informative imaging features to quantify the differences that oncologic tissues exhibit in medical imaging.

Radiomics applies advanced computational methodologies to medical imaging data, to convert medical images into quantitative descriptors of oncologic tissues ${ }^{8}$.

In this study, we analyzed 440 radiomic features quantifying tumor phenotypic differences based on its image intensity, shape and texture. In a large dataset of 1019 lung and head and neck cancer patients, of which we extracted radiomic features on computed tomography images, we found that a large number of radiomic features have prognostic power, many of which its prognostic implication have not been described before. Furthermore, our integrated analysis showed that features selected based on their stability and reproducibility were also the most informative features, which indicates the power of integrating independent datasets for radiomic feature selection and model building.

We showed as well that a radiomic signature, capturing intra-tumor heterogeneity, was strongly prognostic and validated in three independent datasets of lung and head and neck cancer patients, and was associated with gene-expression profiles. To avoid any form of over-fitting or bias, we performed a robust statistical validation: only one 
radiomics signature (containing 4 radiomic features) was validated in data of 545 patients in independent validation datasets (Figure 2 and Extended Data Figure 3). The four features were selected based on feature stability and prognostic performance in the discovery dataset only.

The top performing feature "Gray Level Nonuniformity" (Group3, number 3.25) and the most dominant features in the radiomic signature (feature III and IV), quantified intra-tumor heterogeneity. Indeed, it is often hypothesized that intra-tumor heterogeneity is exhibited on different spatial scales, for example at the radiological, macroscopic, cellular, and the molecular (genetics) level. Radiological tumor phenotype characteristics may thus be useful to investigate the underlying evolving biology. It is known that multiple subclonal populations co-exist within tumors, reflecting extensive intra-tumoral "somatic evolution" ${ }^{15,16}$. This heterogeneity is a clear barrier to the goal of personalized therapy based on molecular biopsy-based assays, as the identified mutations and geneexpression does not always represent the entire population of tumor cells ${ }^{17,18}$. Radiomics circumvents this by assessing the comprehensive 3D tumor bulk. The study presented here probes heterogeneity and demonstrates corresponding clinical importance in two cancer types. Furthermore, we demonstrated association of intra-tumor heterogeneity with proliferation, a general hallmark of cancer.

Overall, the lung-derived radiomic signature had better performance in head and neck compared to lung cancer. One reason could be that head and neck images were acquired with head immobilization, whereas lung images were acquired with freebreathing and are affected by patient movement or respiration, resulting in relatively more image noise. Nonetheless, our results show that the radiomic signature could be transferred from lung to head and neck cancer, which suggests that the signature identifies a general prognostic tumor phenotype.

Our method provides a non-invasive (and therefore with no risk of infection or complications that accompany tissue biopsies), fast, low cost, and repeatable way of investigating phenotypic information, potentially speeding up the development of personalized medicine. Furthermore, we show that the radiomic signature is significantly associated with the underlying gene-expression patterns, suggesting that inter-patient differences of gene-expression are large than intra-patient differences.

The clinical impact of our results are illustrated by the fact that it advances knowledge in the analysis and characterization of tumors in medical images, previously not done, and provides knowledge currently not used in the clinic. We showed the complementary performance of Radiomic features with TNM staging for prediction of outcome, which illustrates the clinical importance of our findings as TNM is routinely used in the clinic. Currently, the TNM staging system is used for risk stratification and treatment decision-making. However, the TNM staging system is primarily based on resectability of the tumor, while a larger number of NSCLC patients will receive primary treatment with radiotherapy either alone or combined with chemotherapy. Therefore, the TNM staging system is insufficient for risk stratification of this group of patients, in particular to make the decision between curative treatment (concomitant radio- 
chemotherapy) or palliative treatment especially in elderly patients, a growing issue in western countries. Our results show that the radiomics signature is performing better in independent cohorts than the TNM classification. In future clinical trials this inexpensive method can be used as well for pretreatment risk stratification (e.g. high, low risk).

Furthermore, we have shown for the first time the translational capability of radiomics in two cancer types (lung and head and neck cancer). These results indicate that radiomics quantifies a general prognostic cancer phenotype that likely can broadly be applied to other cancer types. Similar observations have been made in gene-expression studies where signatures are prognostic across different diseases ${ }^{19}$.

Analysis of image features applied to medical imaging has been a largely studied field and extensive literature exists. However, the majority of previous work describes the use of imaging features focused in the detection of small nodules in for example mammograms or chest CT/PET scans, or in the differential diagnosis of malignant versus benign nodules (Computed Aided Diagnostics). However, applications and methodologies are distinct from our study. Quantitative imaging for personalized medicine is a recent field, with a limited number of publications ${ }^{12,20-27}$. The main clinical question of this research is not the diagnosis, but how to extract more useful information from the tumor phenotype that can be used for personalized medicine.

Therefore, we assessed the association of radiomics with clinical factors, prognosis, and gene-expression levels, using large amounts of features and with external and independent validation cohorts of patients. The most important message in our manuscript is that there is prognostic and biologic information enclosed in routinely acquired CT imaging and was evident in two cancer types.

It is known that variability in image acquisition exists across hospitals and that this is a reality in clinical practice. However, in our analysis we used data directly generated from the scanner and the features were calculated from the RAW imaging data, without any pre-processing or normalization. As there was no correction by cohort or scanner type, this illustrates the translational potential of our results and it is a strong argument in favor of a multi-centric application of radiomics. The radiomics signature had strong prognostic power in these independent datasets generated in daily clinical practice. Furthermore, we expect that with better standardization and imaging protocols, the power of radiomics will even further improve. Among others, the Quantitative Imaging Network (QIN) of the National Institute of Health $(\mathrm{NIH})$, as well as the quantitative imaging biomarker alliance (QIBA), investigates future directions, by performing phantom studies and discussing with vendor's open and standardized protocols for image acquisition $^{2,3}$.

Due to the large availability of non-invasive imaging performed routinely in a large number of cancer patients, and the automated feature algorithms, the results of this work could stimulate further research of image-based quantitative features. Also, we presented evidence that the defined radiomic feature-metrics are platform independent, though this should be studied further, and can potentially be applied to other image modalities, such as magnetic resonance imaging (MRI), or positron emission tomog- 
raphy (PET). This approach can have a large impact as imaging is routinely used in clinical practice, worldwide, in all stages of diagnoses and treatment, providing an unprecedented opportunity to improve medical decision support.

\section{METHODS}

Radiomics Features: In Supplement I the algorithms of radiomic features are described in detail. In short, we defined 440 radiomic image features that describe tumor characteristics and can be extracted in an automated way. In total 440 distinct features

were defined, divided in four groups: I) tumor intensity, II) shape, III) texture, and IV) wavelet features. The first group quantified tumor intensity characteristics using firstorder statistics, calculated from the histogram of all tumor voxel intensity values. Group 2 consists of features based on the shape of the tumor (e.g. sphericity or compactness of the tumor). Group 3 consists of textual features that are able to quantify intra-tumor heterogeneity differences in the texture that is observable within the tumor volume. These features are calculated in all 3-dimensional directions within the tumor volume, thereby taking the spatial location of each voxel compared to the surrounding voxels into account.

Group 4 calculates intensity and textural features from wavelet decompositions of the original image, thereby focusing the features on different frequency ranges within the tumor volume. All feature algorithms were implemented in Matlab.

Datasets: In Supplementary II the datasets are described in detail. In short, we applied a radiomic analysis to six image datasets (see overview in Fig. 2).

- RIDER: This dataset consists of 31 non-small cell lung cancer (NSCLC) patients with two CT-scans acquired approximately 15 min apart ${ }^{10}$. We used this dataset to assess stability of the features for test retest.

- Multiple Delineation: This dataset consists of 21 NSCLC patients were the tumor volume was delineated manually on CT/PET scans by five independent oncologists ${ }^{11}$. We used this dataset to assess stability of the features for delineation inaccuracies.

- Lung1: This datasets consist of 422 NSCLC patients that were treated at MAASTRO Clinic, The Netherlands. For these patients CT scans, manual delineations, clinical and survival data was available. We used this dataset to assess the prognostic value of the radiomic features and to build a radiomic signature.

- Lung 2: This datasets consists of 225 NSCLC patients that were treated at Radboud University Nijmegen Medical Centre, The Netherlands. For these patients CT-scans, manual delineations, clinical, and survival data was available. We used this dataset to validate the prognostic value of the radiomic features and signature in an independent NSCLC cohort. 
- H\&N1: This dataset consists of 136 head and neck squamous cell carcinoma (HNSCC) patients treated at MAASTRO Clinic, The Netherlands. For these patients CT-scans, manual delineations, clinical, and survival data was available. We used this dataset to validate the prognostic value of the radiomic features and signature in HNSCC patients.

- H\&N2: This dataset consists of 95 HNSCC patients treated at the VU Univeristy Medical Center Amsterdam, The Netherlands. For these patients CT-scans, manual delineations, clinical, and survival data was available. We used this dataset to validate the prognostic value of the radiomic features and signature in a second cohort of HNSCC patients.

- Lung 3: This dataset consists of 89 NSCLC patients that were treated at MAASTRO Clinic, The Netherlands. For these patients pre-treatment CT-scans, tumor delineations and gene expression profiles were available. We used this dataset to associate imaging features with gene-expression profiles.

The discovery Lung1 dataset, consisting of CT images for 422 NSCLC patients, and the Lung3 dataset consisting of CT images and gene-expression profiling for 89 NSCLC patients, are publicly available at www.cancerdata.org.

Data Analysis: An overview of the analysis is shown in Figure 2. The analysis was divided in training and validation phases. For the training phase, we first explored feature stability determined in both test-retest and inter-observer setting. The RIDER and Multiple Delineation datasets were used to assess stability of the features to select the most informative features for further investigation. Using the RIDER test retest dataset, we tested the stability of the radiomic features between test and retest ${ }^{10}$. For each patient, we extracted the radiomic features from both scans. A stability rank was calculated for each feature, using the intra-class correlation coefficient (ICC), where a lower ICC rank corresponds to a more stable feature.

We assessed the feature stability for delineation inaccuracies using a Multiple Delineation dataset ${ }^{11}$. All radiomic features were computed for five delineations per patient, and a stability rank per feature was calculated using the Friedman test. The Friedman test is a non-parametric repeated measurement test for a non-Gaussian population. A rank of 1 indicated the most stable feature for delineation inaccuracies and 440 the least stable feature.

All 440 radiomic features were extracted for the Lung1, Lung2, H\&N1, and H\&N2 datasets. The radiomic features were not normalized on any dataset, and only the raw values were used that were directly computed from the DICOM image.

To explore the association of the radiomics features with survival we used KaplanMeier analysis in a training and validation phase. To ensure a completely independent validation, the median threshold of each feature on the Lung1 dataset was computed, and then this threshold was used in the validation datasets (Lung2, H\&N1, and H\&N2) to split the survival curves. We used the G-rho rank test for censored survival data to 
test for significant difference between the two survival curves. P-values were corrected for multiple testing using by controlling the false discovery rate (FDR) of $10 \%$, the expected proportion of false discoveries amongst the rejected hypotheses.

To assess the multivariate performance of radiomic features we build a signature. We selected the 100 most stable features, determined by averaging the stability ranks of RIDER dataset and Multiple Delineation dataset. Next, we computed the performance in the Lung 1 dataset of each of the selected 100 features using the concordance index $(\mathrm{CI})^{12}$. This measure is comparable to the Area Under the Curve (AUC) but can also be used for Cox regression analysis. From each of the four feature groups, we selected the single best performing feature for prognosis in the Lung1 dataset, and combined these top four features into a multivariate Cox proportional hazards regression model for prediction survival. The weights of the model were fitted on the Lung1 dataset. We applied the radiomic signature to the validation datasets Lung2, H\&N1, and H\&N2, and performance was assessed with the $\mathrm{Cl}$. To calculate significance between two models we used a bootstrap approach, for 100 times we calculated the $\mathrm{Cl}$ of both models from 100 random selected samples. The Wilcoxon test was used to assess significance.

A similar approach was used to assess if the signature had significant power, compared with random $(\mathrm{Cl}=0.5)$. We used a bootstrap approach, for 100 times we calculated the $\mathrm{Cl}$ of the radiomics signature based on 100 random selected samples with correct outcome data, as well as on 100 random chosen samples with random outcome data. This process was repeated 100 times. The Wilcoxon test was used to assess significance, between the two distributions.

To assess the complementary effect of the signature with clinical parameters, we build a new model with the prediction of the signature as one input and the clinical parameter as the other input. The weight of the clinical parameter was fitted on the training dataset Lung1.

To assess the association of the radiomic signature with gene expression we used the Lung3 dataset. Gene expression of 89 patients was measured on Affymetrix chips with the custom chipset HuRSTA_2a520709 for 21766 genes. Expression values were normalized with the RMA algorithm5 in the Affy package in Bioconductor. For each of the four features in the radiomic signature, we calculated the Spearman rank correlation to gene expression and used the corresponding $p$-values to obtain a rank of genes representing high to low agreement. Each of these gene ranks were used to perform a pre-ranked version of Gene Set Enrichment Analysis (GSEA) ${ }^{14}$ on the C5 collection of $M S i g D B^{28}$, which contains gene sets associated with specific $G O$ terms. We only regarded gene sets of size 15 to 500. Local false-discovery-rates were calculated on the normalized enrichment scores (NES), GSEA's primary statistic, and only gene sets enriched with an FDR of $<=20 \%$ were retained. Fig. $4 \mathrm{~B}$ displays gene sets that have been significantly enriched (FDR $<=20 \%$ ) for at least one of four radiomic features (indicated by an asterisk). The corresponding absolute NES in all of the four features are given colorcoded, where light blue means low and dark blue means high NES. 


\section{ACKNOWLEDGMENTS}

Authors acknowledge financial support from the National Institute of Health (NIH-USA U01 CA 143062-01, Radiomics of NSCLC), the CTMM framework (AIRFORCE project, grant 030-103), EU 6th and 7th framework program (METOXIA, EURECA, ARTFORCE), euroCAT (IVA Interreg - www.eurocat.info), and the Dutch Cancer Society (KWF UM 2011-5020, KWF UM 2009-4454). Authors also acknowledge financial support from the QuIC-ConCePT project (Grant Agreement No. 115151).

\section{SUPPLEMENTARY INFORMATION}

http://www.nature.com/article-assets/npg/ncomms/2014/140603/ncomms5006/extref/ ncomms5006-s1.pdf 


\section{REFERENCES}

1. Kurland, B. F. et al. Promise and pitfalls of quantitative imaging in oncology clinical trials. Magn Reson Imaging 30, 1301-1312 (2012).

2. Buckler, A. J., Bresolin, L., Dunnick, N. R., Sullivan, D. C.Group. A collaborative enterprise for multistakeholder participation in the advancement of quantitative imaging. Radiology 258, 906-914 (2011).

3. Buckler, A. J. et al. Quantitative imaging test approval and biomarker qualification: interrelated but distinct activities. Radiology 259, 875-884 (2011).

4. Lambin, P. et al. Predicting outcomes in radiation oncology-multifactorial decision support systems. Nat Rev Clin Oncol 10, 27-40 (2013).

5. Jaffe, C. C. Measures of response: RECIST, WHO, and new alternatives. J Clin Oncol 24, 3245-3251 (2006).

6. Burton, A. RECIST: right time to renovate? The Lancet Oncology 8, 464-465 (2007).

7. Birchard, K. R., Hoang, J. K., Herndon, J. E. \& Patz, E. F. Early changes in tumor size in patients treated for advanced stage nonsmall cell lung cancer do not correlate with survival. Cancer 115, 581-586 (2009).

8. Lambin, P. et al. Radiomics: extracting more information from medical images using advanced feature analysis. Eur. J. Cancer 48, 441-446 (2012).

9. Kumar, V. et al. Radiomics: the process and the challenges. Magn Reson Imaging 30, 1234-1248 (2012).

10. Zhao, B. et al. Evaluating Variability in Tumor Measurements from Same-day Repeat CT Scans of Patients with Non-Small Cell Lung Cancer. Radiology 252, 263-272 (2009).

11. van Baardwijk, A. et al. PET-CT-based auto-contouring in non-small-cell lung cancer correlates with pathology and reduces interobserver variability in the delineation of the primary tumor and involved nodal volumes. International journal of radiation oncology, biology, physics 68, 771-778 (2007).

12. Harrell, F. E. Regression modeling strategies: with applications to linear models, logistic regression, and survival analysis. (2001).

13. Compton, C. C. et al. AJCC Cancer Staging Atlas. (Springer, 2012).

14. Subramanian, A. Gene set enrichment analysis: A knowledge-based approach for interpreting genomewide expression profiles. Proc Natl Acad Sci USA 102, 15545- 15550 (2005).

15. Yachida, S. et al. Distant metastasis occurs late during the genetic evolution of pancreatic cancer. Nature 467, 1114-1117 (2010).

16. Gerlinger, M. et al. Intratumor heterogeneity and branched evolution revealed by multiregion sequencing. N Engl J Med 366, 883-892 (2012).

17. Gerlinger, M. \& Swanton, C. How Darwinian models inform therapeutic failure initiated by clonal heterogeneity in cancer medicine. Br. J. Cancer 103, 1139-1143 (2010).

18. Kern, S. E. Why your new cancer biomarker may never work: recurrent patterns and remarkable diversity in biomarker failures. Cancer Res. 72, 6097-6101 (2012).

19. Starmans, M. H. W. et al. Independent and functional validation of a multi-tumourtype proliferation signature. Br. J. Cancer 107, 508-515 (2012).

20. Nair, V. S. et al. Prognostic PET 18F-FDG uptake imaging features are associated with major oncogenomic alterations in patients with resected non-small cell lung cancer. Cancer Res. 72, 3725-3734 (2012).

21. Diehn, M. et al. Identification of noninvasive imaging surrogates for brain tumor gene-expression modules. Proc Natl Acad Sci USA 105, 5213-5218 (2008).

22. Segal, E. et al. Decoding global gene expression programs in liver cancer by noninvasive imaging. Nat Biotechnol 25, 675-680 (2007).

23. Tixier, F. et al. Intratumor heterogeneity characterized by textural features on baseline $18 \mathrm{~F}-\mathrm{FDG}$ PET images predicts response to concomitant radiochemotherapy in esophageal cancer. J Nucl Med 52, 369378 (2011).

24. Naqa, El, I. et al. Exploring feature-based approaches in PET images for predicting cancer treatment outcomes. Pattern Recognit 42, 1162-1171 (2009).

25. Ganeshan, B., Panayiotou, E., Burnand, K., Dizdarevic, S. \& Miles, K. Tumour heterogeneity in non-small cell lung carcinoma assessed by CT texture analysis: a potential marker of survival. European Radiology 22, 796-802 (2011). 
26. Ganeshan, B., Skogen, K., Pressney, I., Coutroubis, D. \& Miles, K. Tumour heterogeneity in oesophageal cancer assessed by CT texture analysis: Preliminary evidence of an association with tumour metabolism, stage, and survival. Clinical Radiology 67, 157-164 (2012).

27. Gevaert, O. et al. Non-small cell lung cancer: identifying prognostic imaging biomarkers by leveraging public gene expression microarray data--methods and preliminary results. Radiology 264, 387-396 (2012).

28. Liberzon, A. et al. Molecular signatures database (MSigDB) 3.0. Bioinformatics 27, 1739-1740 (2011). 



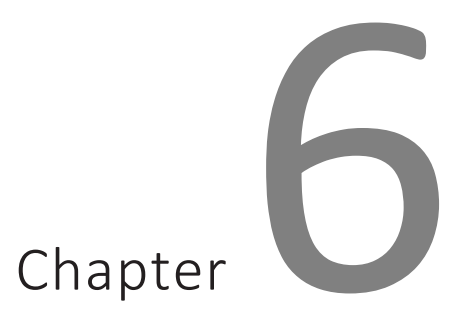

\section{Radiomic feature clusters and prognostic signatures specific for Lung and Head \& Neck cancer}

Published in: Nature Scientific Reports 5, 11044; 2015.

Radiomic feature clusters and prognostic signatures specific for Lung and Head \& Neck cancer

Chintan Parmar*, Ralph T.H. Leijenaar*, Patrick Grossmann, Emmanuel Rios Velazquez, Johan Bussink, Derek Rietveld, Michelle M. Rietbergen, Benjamin Haibe-Kains, Philippe Lambin, Hugo J.W.L. Aerts

*These authors contributed equally to this work 


\section{ABSTRACT}

Radiomics provides a comprehensive quantification of tumor phenotypes by extracting and mining large number of quantitative image features. To reduce the redundancy and compare the prognostic characteristics of radiomic features across cancer types, we investigated cancer-specific radiomic feature clusters in four independent Lung and Head \& Neck (H\&N) cancer cohorts (in total 878 patients). Radiomic features were extracted from the pre-treatment computed tomography (CT) images. Consensus clustering resulted in eleven and thirteen stable radiomic feature clusters for Lung and $\mathrm{H} \& \mathrm{~N}$ cancer, respectively. These clusters were validated in independent external validation cohorts using rand statistic (Lung RS $=0.92, p<0.001, H \& N$ RS $=0.92, p<0.001$ ). Our analysis indicated both common as well as cancer-specific clustering and clinical associations of radiomic features. Strongest associations with clinical parameters: Prognosis Lung $\mathrm{Cl}=0.60 \pm 0.01$, Prognosis $\mathrm{H} \& \mathrm{~N} \mathrm{Cl}=0.68 \pm 0.01$; Lung histology $\mathrm{AUC}=0.56 \pm 0.03$, Lung stage $A \cup C=0.61 \pm 0.01, \mathrm{H} \& N$ HPV AUC $=0.58 \pm 0.03, \mathrm{H} \& N$ stage $A U C=0.77 \pm$ 0.02 . Full utilization of these cancer-specific characteristics of image features may further improve radiomic biomarkers, providing a non-invasive way of quantifying and monitoring tumor phenotypic characteristics in clinical practice. 


\section{INTRODUCTION}

Recent advances of medical and computational science have led to the emergence of 'precision medicine', which has revolutionized the cancer care and medical science in general. A major proportion of precision medicine research has centered on unveiling different molecular characteristics of the disease tissues by using genomic and proteomic technologies. In spite of their enormous potential, these techniques have found limited implementations in routine clinical practice ${ }^{1}$. A major challenge being the invasive nature, as biopsies, having high associated risk and cost, are often required.

Imaging on the other hand provides promising means of non-invasive tissue characterization and is furthermore routinely used for disease detection, diagnosis, and treatment purposes in clinical practice ${ }^{2-4}$. X-ray computed tomography (CT) is a frequently used imaging modality for oncology because it assesses tissue density in high resolution and exhibits strong contrasts among different tissue types. In routine clinical practice, tumor response to therapy is measured by the RECIST and/or WHO criteria, based on CT imaging. These descriptors measure the change in size of tumors, and often do not succeed in predicting overall survival ${ }^{5,6}$.

"Radiomics" is an emerging field of research that aims to utilize the full potential of medical imaging. Radiomics focuses on extracting a large number of quantitative features from medical images, providing a more detailed quantification of tumor phenotypic characteristics-effectively converting medical images into a high dimensional minable feature space ${ }^{7-9}$. Several studies have defined and quantified various image descriptors and stated their significance for treatment monitoring and outcome prediction in different cancer types ${ }^{10-14}$. Moreover, some studies have also reported an association between radiographic imaging phenotypes and tumor stage, metabolism ${ }^{15}$, hypoxia, angiogenesis ${ }^{16}$ and the underlying gene and/or protein expression profiles ${ }^{17-19}$.

A main challenge in radiomics is to deal with feature redundancy in order to obtain a non-redundant set of imaging biomarkers. Consensus clustering ${ }^{20}$ could address this issue by reducing the feature space into several non-redundant feature clusters. In this study we identified and validated radiomic feature clusters in cohorts of Lung cancer and Head \& Neck (H\&N) cancer patients. We also evaluated the clinical importance of these clusters by quantifying their association with important clinical parameters and patient survival. Moreover, we used the identified radiomic clusters to build cancerspecific multivariable radiomic signatures and tested their prognostic performance. Identification of cancer-specific radiomic clusters provides a crucial step towards stable and clinically relevant radiomic biomarkers, providing a non-invasive way of quantifying and monitoring tumor phenotypic characteristics in clinical practice. 


\section{METHODS}

\section{Radiomic features}

We defined 440 radiomic image features that quantify tumor characteristics. These features were divided in four groups: I) tumor intensity, II) shape, III) texture and IV) wavelet features. Tumor intensity based features, which are defined using first order statistics of the intensity histogram, quantified the density of the tumor region on CT image. Shape features described the 3D geometric properties of the tumor, whereas textural features quantified intra-tumor heterogeneity. Textural features were computed by analyzing the spatial distribution of voxel intensities in thirteen directions. These features are derived from gray level co-occurrence $(\mathrm{GLCM})^{21}$ and run length matrices $(G L R L M)^{22}$ and were computed by averaging their values over all thirteen directions. Wavelet features are the transformed domain representations of the intensity and textural features. These features were computed on different wavelet decompositions of the original image using a coiflet wavelet transformation. All image analysis was performed in Matlab R2012b (The Mathworks, Natick, MA) using an adapted version of CERR (Computational Environment for Radiotherapy Research) ${ }^{23}$ and features were automatically extracted with in-house developed radiomics image analysis software. Mathematical definitions of all radiomic features as well as the extraction methods were previously described ${ }^{18}$.

\section{Datasets}

Briefly, we considered four image datasets (see overview in Figure 1) for this study, from different institutes in the Netherlands: (1) Lung1: 422 NSCLC patients treated at MAASTRO Clinic in Maastricht. (2) Lung2: 225 NSCLC patients treated at Radboud University Medical Centre in Nijmegen. (3) HN1: 136 head and neck squamous cell carcinoma (HNSCC) patients treated at MAASTRO Clinic in Maastricht and (4) HN2: 95 HNSCC patients treated at the VU University Medical Centre in Amsterdam. CT-scans, manual delineations and clinical data were available for all included patients. More details on the included datasets have been described earlier ${ }^{18}$.

\section{Data analysis}

Comparison of the prognostic performance of radiomic features in Lung and H\&N cancer In order to compare the prognostic utility of radiomic features across Lung and H\&N cancer, for each feature, we computed and compared the concordance index $(\mathrm{Cl})^{24}$, which is the generalization of area under ROC curve. R package survcomp was used for the analysis ${ }^{25}$. P-values are corrected for multiple testing (FDR 5\%). 


\section{Consensus clustering}

We used consensus clustering to cluster the radiomic features extracted from the training cohorts Lung1 and HN1. Consensus clustering is a resampling based clustering methodology, which quantifies the consensus between several clustering iterations and provides means to estimate the number of clusters that best fit the data ${ }^{20}$. We estimated the range for the appropriate number of clusters from the delta area plots (supplementary figure S1). From this range, we chose the number of clusters, which gave the highest median cluster consensus over all clusters. Cluster consensus was defined as the average consensus between all pairs of features belonging to the same cluster. Cluster consensus (range [0-1]) indicates the robustness (stability) of a cluster over resampling. We also computed the mean pairwise correlation (range [0-1]) between features of a cluster, which is a measure of the cluster compactness (similarity of features within the cluster). Qualitative categorization of cluster stability was defined as; consensus $<0.5$, poor stability; $0.5 \leq$ consensus $<0.75$, moderate stability; and consensus $\geq 0.75$, high stability. Cluster compactness was also assessed using the same qualitative categorization. We applied hierarchical clustering with agglomerative ward linkage, a Pearson correlation based dissimilarity measure $(1-r)$ and 10,000 resampling iterations. Consensus clustering was performed using the R package ConsensusClusterPlus ${ }^{26}$.

\section{Radiomic cluster validation}

Radiomic feature clusters obtained on the training cohorts, Lung1 and HN1, were considered as the reference Lung and $H \& N$ clusters. For cluster validation, we clustered the Lung and $\mathrm{H} \& \mathrm{~N}$ validation cohorts, Lung2 and HN2, using the same hierarchical clustering algorithm and the same number of clusters as for the corresponding training cohort. Rand Statistic (RS) ${ }^{27}$ was used to assess the agreement between each reference clustering $(P)$ and the clustering obtained for its respective validation cohort $(C)$ and was defined as:

$$
R S=\frac{|S S|+|D D|}{|S S|+|S D|+|D S|+|D D|}
$$

where $|S S|$ is the number of feature pairs that cluster together in both $C$ and $P,|S D|$ is the number of feature pairs that cluster together in $C$ but not in $P,|D S|$ is the number of feature pairs that cluster together in $\mathrm{P}$, but not in $\mathrm{C}$ and $\mid D D /$ is the number of feature pairs that do not cluster together in both $\mathrm{C}$ and $\mathrm{P}$. Significance of RS was determined by a random permutation test using 1000 iterations.

\section{Similarity between Lung and H\&N clusters}

Cluster overlap between the individual feature clusters of Lung and H\&N radiomic cohorts were assessed using the Jaccard index ${ }^{27}$, which is defined as: 


$$
\operatorname{Jaccard}(L, H)=\frac{|L \bigcap H|}{|L \bigcup H|}
$$

where $L$ and $H$ are any feature clusters of Lung and $H \& N$ cohorts. Qualitative categorization of cluster overlap was defined as; Jaccard $<0.5$, poor overlap; $0.5 \leq$ Jaccard $<0.75$, moderate overlap; and Jaccard $\geq 0.75$ high overlap.

\section{Clinical relevance of radiomic clusters}

In order to quantify the association between radiomic feature clusters and patient survival, we used the concordance index $(\mathrm{Cl})$, whereas the association between a feature cluster and a categorical clinical parameter (i.e. Lung cancer histology, H\&N HPV status or Lung/H\&N tumor stage) was quantified using the area under the ROC curve (AUC). For clinical parameters having more than two categorical levels, a multi-class AUC was computed using a pairwise approach. Univariable $\mathrm{Cl}$ and $\mathrm{AUC}$ were computed for each feature. A cluster's association with patient survival and clinical parameters was then quantified as the mean $\mathrm{Cl}$ and mean AUC over all contained features. Significance was estimated using a random permutation test with 1000 iterations. R package survcomp ${ }^{25}$ and $\mathrm{pROC}^{28}$ was used for this analysis. Qualitative categorization of the prognostic or predictive performance was defined as poor $(\mathrm{Cl}$ or $\mathrm{AUC}<0.6)$, moderate $(0.6 \leq \mathrm{Cl}$ or $A \cup C<0.75)$ and high $(\mathrm{Cl}$ or $\mathrm{AUC} \geq 0.75)$.

\section{Multivariable clinical relevance}

In order to select non-redundant imaging biomarkers, cancer-specific radiomic signatures were built using the medoids of the obtained clusters of Lung1 and HN1 cohorts. The medoid is a single representative feature, which has the highest average pairwise correlation within a cluster. To investigate the multivariable prognostic utility of these selected radiomic features, a multivariable Cox proportional hazards model was fitted on each training cohort (i.e. Lung1 and HN1) and their prognostic performance was tested on validation cohorts (i.e. Lung2 and $\mathrm{HN} 2$ ) using the $\mathrm{Cl}$. R package survcomp ${ }^{25}$ was used for this analysis. For the prediction of categorical clinical parameters, we built multivariable classifiers on each training cohort (i.e. Lung1 or HN1) using logistic regression, with the medoids as independent variables. The predictive performance of a classifier was evaluated on the corresponding validation cohort (Lung2 or HN2) using AUC. For clinical parameters having more than two categorical levels, logistic regression was fitted using a pairwise approach and performance was evaluated using multiclass $A \cup C^{29}$. $\mathrm{R}$ package $\mathrm{VGAM}^{30}$ was used for this analysis. 


\section{RESULTS}

In order to investigate radiomic features in Lung and H\&N cancer cohorts, a total of 440 radiomic features were extracted from the segmented tumor regions of the pretreatment CT images of Lung and Head and Neck cancer cohorts. In our analysis, we used datasets Lung1 $(n=422)$ and HN1 $(n=136)$ as training datasets, and Lung2 ( $n=225)$ and HN2 ( $n=95)$ as validation datasets (Figure 1).

(a)
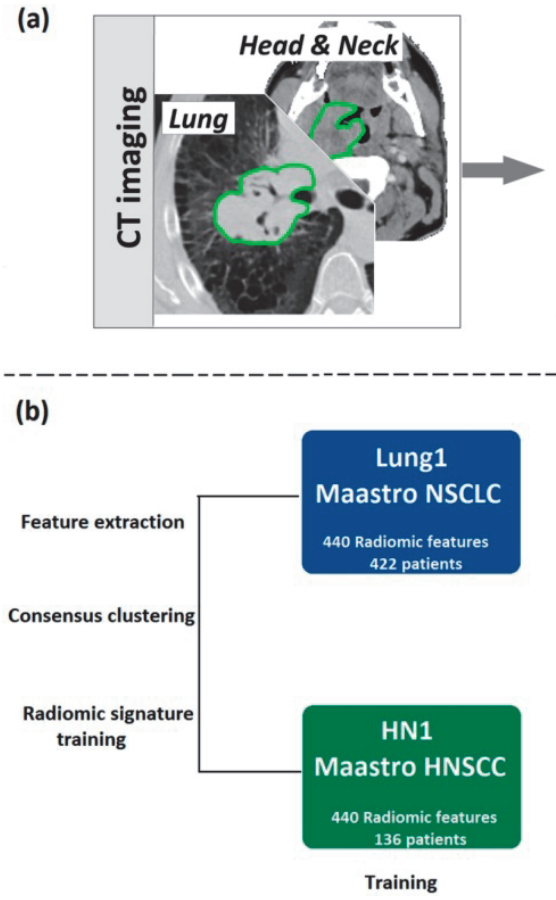
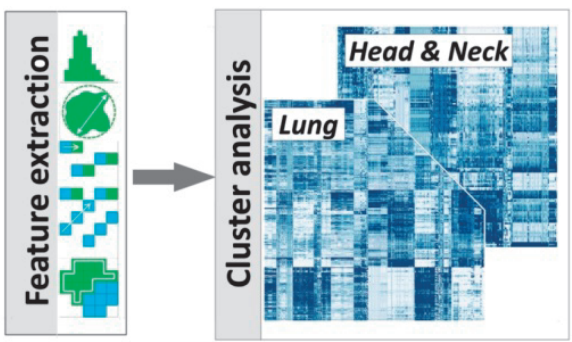

Figure 1. (a) Radiomic analysis overview: For both Lung and H\&N cancer datasets, we extracted radiomic features from pre-treatment CT images. Cluster analysis was performed on the feature data. (b) Datasets overview: Four independent radiomic cohorts of Lung and Head \& Neck cancer were included in the analysis. Lung1 and HN1 were used for training; Lung2 and HN2 were used for validation.

\section{Comparison of the prognostic performance of radiomic features in Lung and} H\&N cancer

The prognostic utility of the radiomic features was assessed using the concordance index $(\mathrm{Cl})$. Figure 2 depicts a $\mathrm{Cl}$ heatmap of radiomic features in the validation cohorts (Lung2 and HN2). We observed that 143 features had significant prognostic performance $(\mathrm{Cl}>0.5, \mathrm{p}<0.05$ FDR corrected) in both the cancer types whereas 212 features (190 features in Lung and 22 features in H\&N) showed significant prognosis only in one of the two cancer types. Eighty-five features turned out not to be significantly prognostic in either of the two cancer types. 
- Significant in both

- Significant in Lung only

- Significant in HN only

- not Significant
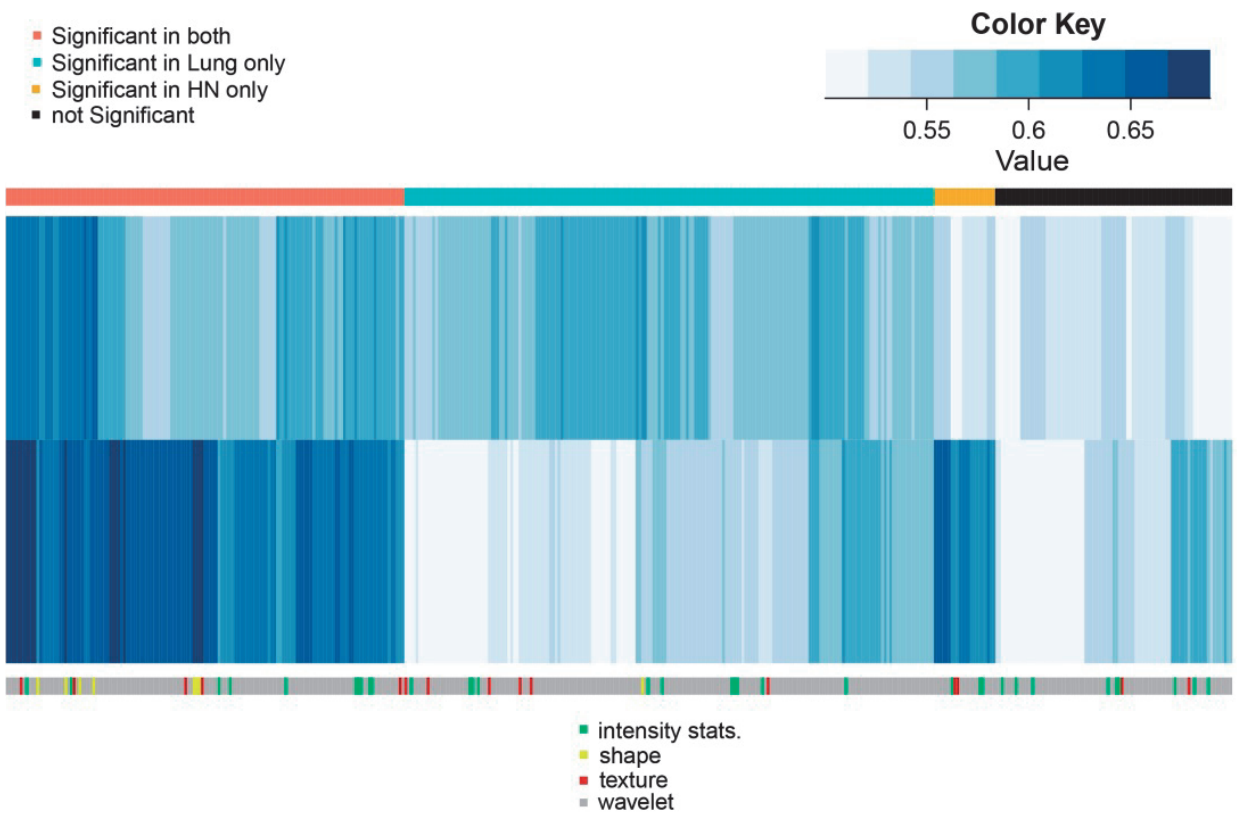

Figure 2. Heatmap showing the prognostic performance of radiomic features in Lung2 and HN2 cohorts. Prognostic performance was evaluated using the concordance index $(\mathrm{Cl})$. Note that a large number of features are prognostic in both cancer types. However, also a large number of features are cancer-type specific, e.g. prognostic only in Lung or only in H\&N cancer.

\section{Identification and validation of radiomic feature clusters in Lung cancer}

In order to identify stable clusters of radiomic features, consensus clustering procedure was applied on Lung1 training cohort. We obtained eleven distinct clusters (size: 16 to 65 features per cluster). Heatmaps in Figure 3 depict the consensus maps (Figure 3a) and normalized expression levels (Figure 3c) of the obtained Lung radiomic feature clusters in Lung1 training cohort. These clusters were validated in the Lung2 validation cohort (RS $=0.92$, permutation test $p$-value $<0.001$ ). We observed that four clusters (LCL-4, LCL-6, LCL-7, LCL-11) had a high cluster consensus (consensus $\geq 0.75$ ) and within cluster correlation (correlation $\geq 0.75$ ), whereas six clusters (LCL-1, LCL-2, LCL-3, LCL-5, LCL-8, LCL-10) showed high cluster consensus (consensus $\geq 0.75$ ) but moderate within cluster correlation $(0.5 \leq$ correlation $<0.75)$. For cluster $L C L-9$, both the cluster consensus and within cluster correlation were poor (consensus $=0.41$, correlation $=0.14$ ). Details regarding the cluster size, associated feature categories, cluster consensus and within cluster correlation can be obtained from Table 1.

Identification and validation of radiomic feature clusters in HN cancer

Consensus clustering in HN1 training cohort resulted in thirteen distinct radiomic feature clusters (size: 8 to 93 features per cluster), which were validated in independent 
HN2 validation cohort ( $R S=0.92$, permutation test $p$-value $<0.001)$. Heatmaps in Figure $3 \mathrm{~b}$ and $3 \mathrm{~d}$ show the consensus maps and normalized expression levels of the obtained radiomic feature clusters in HN1 training cohort. Six clusters (HNCL-1, HNCL-2, HNCL-6, HNCL-7, HNCL-12, HNCL-13) had high cluster consensus (consensus $\geq 0.75$ ) and within cluster correlation (correlation $\geq 0.75$ ), whereas five other clusters (HNCL-4, HNCL-5, HNCL-8, HNCL-9, HNCL-11) showed high cluster consensus (consensus $\geq 0.75$ ) but moderate within cluster correlation $(0.5 \leq$ correlation $<0.75)$. Cluster HNCL-10 had moderate cluster consensus (consensus $=0.65$ ) and poor cluster correlation (correlation = 0.04 ), whereas clusters HNCL-3 showed poor cluster consensus (consensus $=0.41$ ) and correlation (correlation $=0.12)$ (see Table 2$)$.

\section{Similarity between Lung and $H \& N$ clusters}

In order to assess the overlap between individual Lung and $\mathrm{H} \& \mathrm{~N}$ radiomic clusters, we compared the Lung and $\mathrm{H} \& \mathrm{~N}$ clusters, pairwise, using the Jaccard index. We observed that Lung cluster LCL-6 and H\&N cluster HNCL-7 had high overlap (Jaccard $=0.98$ ). Cluster pairs LCL-7 \& HNCL-2 (Jaccard = 0.65), LCL-8 \& HNCL-4 (Jaccard = 0.66) and LCL-5 \& HNCL-11 (Jaccard $=0.66$ ) and LCL-3 \& HNCL-8 (Jaccard = 0.66) showed moderate overlap, whereas the remaining pairs had poor overlap (Jaccard < 0.5) (see Figure 4).

\section{Clinical relevance of radiomic clusters}

Mean $\mathrm{Cl}$ and mean AUC values for the obtained Lung and $\mathrm{H} \& \mathrm{~N}$ clusters are depicted in Table 1 \& Table 2. All eleven Lung clusters had a significant prognostic association with patient survival. However, only four Lung clusters (LCL-1, LCL-4, LCL-5 and LCL-6) had a mean $\mathrm{Cl}$ higher or equal to 0.58 . Two Lung clusters (LCL-9 and LCL-10) were significantly associated with tumor histology. All Lung clusters had significant association with tumor stage (see Table 1). For H\&N cancer, six clusters (HNCL-1, HNCL-2, HNCL-6, HNCL-7, HNCL-8 and HNCL-12) were significantly associated with patient survival. We did not observe any association between the H\&N clusters and HPV status. However, except for the three H\&N clusters (HNCL-4, HNCL-10, HNCL-13), all the other H\&N clusters were significantly associated with tumor stage (see Table 2). Univariable $\mathrm{Cl}$ and AUC values of radiomic features in Lung1 and HN1 cohorts are represented by heatmaps in Figure 3e and Figure 3f, respectively.

\section{Multivariable clinical relevance}

We built two radiomic signatures, one each for Lung and H\&N cohort, using the medoids of the obtained Lung and H\&N radiomic feature clusters, respectively. To evaluate the multivariate prognostic performance, we trained a multivariable Cox proportional hazards model on both training cohorts (Lung1 and HN1). Prognostic performance of each model was externally tested on validation cohorts (Lung2 and HN2). We observed 
that the prognostic performance of Lung based multivariable model $(\mathrm{Cl}=0.61)$ was higher than the $\mathrm{H} \& \mathrm{~N}$ model $(\mathrm{Cl}=0.56)$ in Lung2 validation cohort, whereas for HN2 validation cohort, $\mathrm{H} \& \mathrm{~N}$ based multivariable model $(\mathrm{Cl}=0.63)$ performed better than the Lung model $(\mathrm{Cl}=0.57)$. We also used the cluster medoids, for the prediction of clinical parameters. We trained classifiers on training cohort (i.e. Lung1 or HN1) using logistic regression and evaluated the predictive performance in the corresponding validation cohort (Lung2 or HN2). The Lung signature had a moderate performance for the prediction of tumor histology $(A \cup C=0.64)$ and tumor stage $(A \cup C=0.64)$. The $H \& N$ signature was highly predictive for tumor stage $(A \cup C=0.80)$ and had a moderate predictive performance for HPV status (AUC $=0.60$ ).

\section{DISCUSSION}

Medical imaging plays an important role in medical care and science due to its ability to assess tissue characteristics and organ anatomy non-invasively. It is therefore widely used in disease diagnosis, progression assessment and treatment monitoring in clinical oncology. Radiomics, a high throughput approach, can quantify the differences between oncologic tissues and hence provide prognostic or predictive imaging biomarkers ${ }^{7,8}$

In this study we investigated clustering as a means to deal with the high dimensional feature space generated with radiomics, as well as to investigate common and cancertype specific radiomic patterns. We applied consensus clustering on 440 radiomic features extracted from Lung cancer and Head \& Neck cancer patient cohorts. Furthermore these clusters were externally validated on independent validation cohorts. For both cancer types, many clusters showed high cluster consensus and high within cluster correlation, which indicates the high robustness (stability) and compactness of these clusters. These results indicate that consensus clustering could provide robust radiomic feature clusters and hence reduce the feature redundancy. The majority of the obtained Lung and H\&N radiomic clusters were significantly associated with patient survival and tumor stage. Two Lung clusters also showed significant association with tumor histology. Our multivariable analysis showed that cancer-specific multivariable radiomic signatures displayed moderate or high prognostic (predictive) performance.

Comparing the individual Lung and $\mathrm{H} \& \mathrm{~N}$ feature clusters, we observed that five cluster pairs had substantial overlap (Jaccard $\geq 0.6$ ) between the Lung and H\&N cancer, whereas the overlap for other cluster pairs was poor. These results demonstrate both common as well as cancer-specific clustering characteristics of radiomic features.

It can be observed from our analysis that radiomic features also have cancer-specific prognostic ability. We compared the univariable $\mathrm{Cl}$ values of radiomic features across the two cancer types and observed that several radiomic features have significant prognostic utility in only one of the two cancer types. Furthermore the multivariable radiomic signatures performed better in validation cohorts of the same cancer type in our multivariable analysis. 

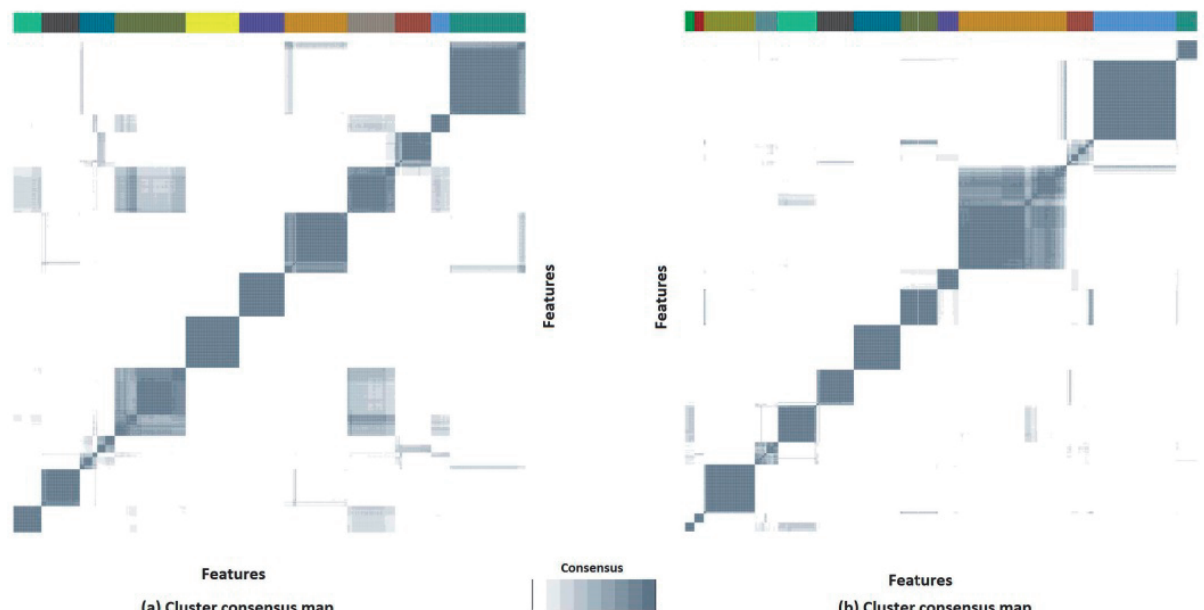

(a) Cluster consensus map

(b) Cluster consensus map
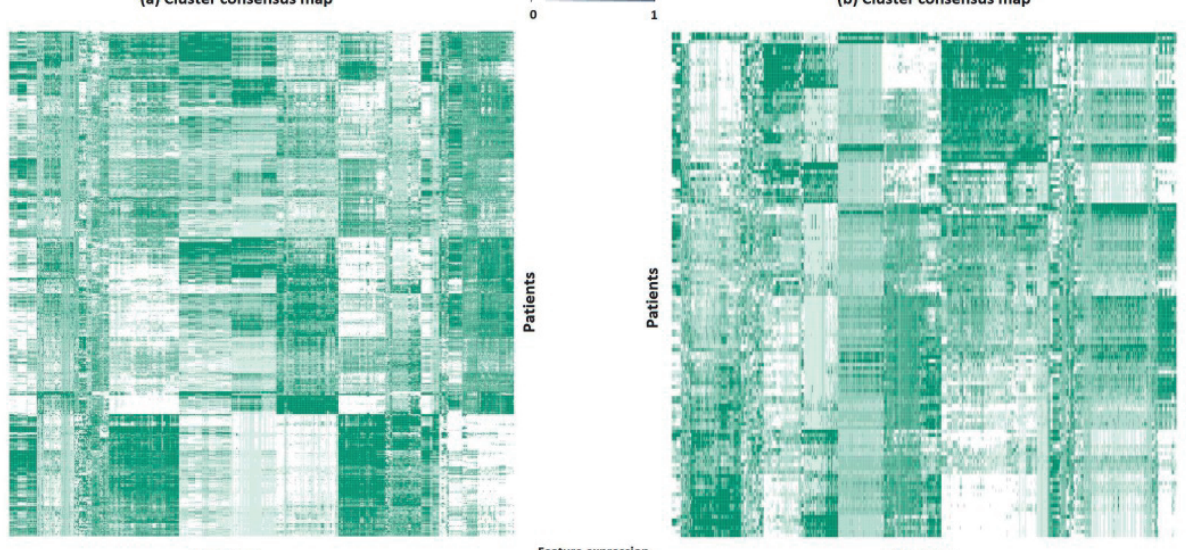

Features

(c) Radiomic feature expression
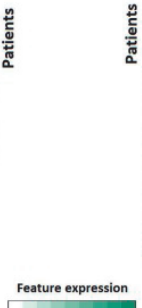

adiomic feature expression
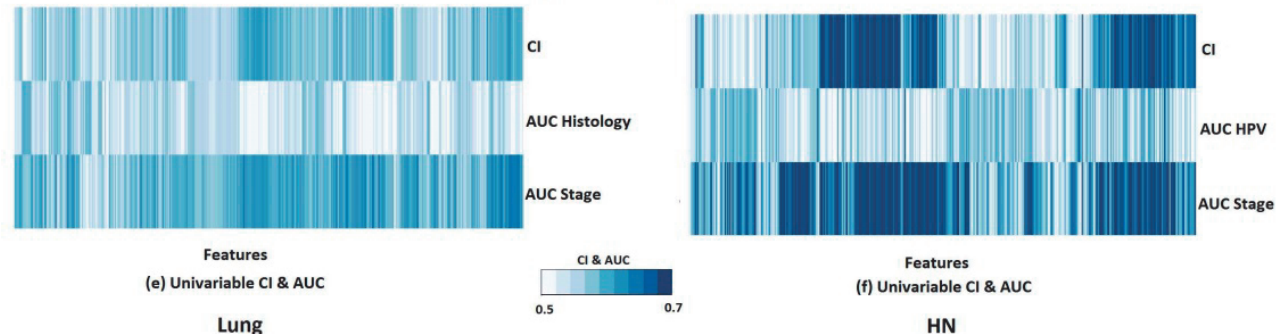

Features

(f) Univariable $\mathrm{Cl}$ \& AUC

HN

Figure 3. Heatmaps for radiomic features of Lung and H\&N training cohorts ordered with respect to the obtained Lung and H\&N clusters. (a-b) Cluster consensus maps of Lung cancer (11 clusters) and H\&N cancer (13 clusters) cohorts. (c-d) Radiomic feature expressions of Lung and H\&N radiomic clusters. (e-f) Clinical relevance $(\mathrm{Cl} \& \mathrm{AUC})$ of radiomic clusters of Lung and $\mathrm{H} \& \mathrm{~N}$ cancer. 


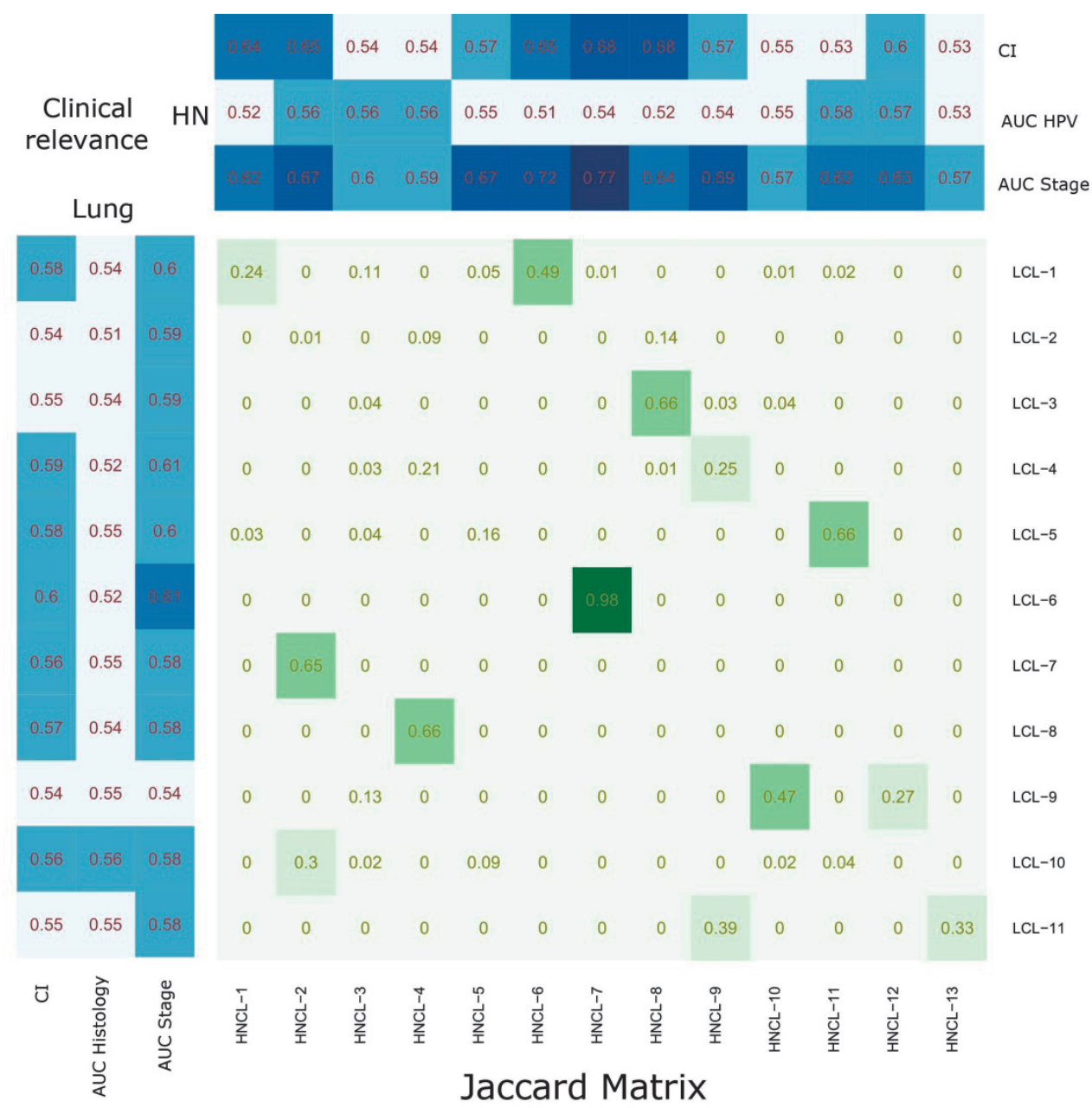

Figure 4. Heatmap depicting cluster overlap and clinical relevance ( $\mathrm{Cl} \& \mathrm{AUC})$. Center matrix in green \& white color represents the overlap (Jaccard index) between the clusters of Lung (rows) and H\&N (columns) radiomic cohorts. Top and left side panels in blue \& white color depicts the average $\mathrm{Cl} \& \mathrm{AUC}$ of the corresponding Lung and $\mathrm{H} \& \mathrm{~N}$ radiomic clusters.

Recently, Aerts et $\mathrm{al}^{18}$, built a radiomic signature of four features, which were selected based on their stability across test-retest image scans and multiple tumor delineations, as well as their prognostic performance in a training dataset. However, the feature selection was only applied to one cancer type (i.e. lung cancer patients). The primary objective of our study was to separately investigate and compare radiomic feature subgroups in Lung and H\&N cancer. Our analysis reveals a cancer-specific grouping and prognostic trends of radiomic features, which could be exploited to potentially improve the performance of prognostic models. In another radiomic study of Lung cancer cohorts, Balagurunathan et $\mathrm{al}^{31}$, used a three step procedure to select the most reproducible, informative and non-redundant features. In this method, it was required to empiri- 
cally choose three threshold values corresponding to the concordance correlation coefficient, dynamic range and pairwise correlation. On the contrary, our method of feature selection only depends on the number of clusters. In general, the estimation of number of clusters is done by a visual inspection of consensus matrices and the delta area plot ${ }^{20}$. However, we used a more deterministic method for the estimation of number of clusters. Overall, the parameters of consensus clustering procedure were carefully chosen, keeping in mind the larger applicability of the obtained results in radiomics. However, these parameters are still just design choices and there may be other relevant parameter configurations, which could be tried and the resultant clustering outputs could be analyzed and compared. These interesting research issues do not fall within the scope of this study, which is primarily focused on the cancer specific comparison of radiomic features.

We expect that the obtained feature clusters of our study combined with feature stability information could further enhance the feature selection process, providing more reliable and prognostic radiomic signatures. However, due to the unavailability of test-retest and multiple delineation $H \& N$ cancer cohorts, we could not compute the stability of H\&N based radiomic features and hence could not include a cancer-specific stability analysis in our study. Machine learning based advanced feature selection methods could also enhance the radiomic biomarker selection process. Although identifying the optimal feature selection method is very important for radiomics research, it does not fit well within the clustering framework of this study and hence should be addressed in future studies, investigating machine learning methods for radiomic data.

We focused only on CT derived radiomic features in this study. However, imaging is routinely performed in clinical practice using different modalities (e.g. CT, PET, MRI, etc.). An integrated radiomic analysis of different cancer types using multiple modalities could therefore provide even more information to facilitate medical decision support with imaging biomarkers.

This study identified different Lung and H\&N radiomic feature subgroups and quantified their clinical significance. Our investigation revealed that clustering and prognostic characteristics of radiomic features are cancer-specific. Cancer-specific prognostic characteristics of radiomic features should be considered for building prognostic models, which could improve the prognosis in cancer care. In general, high throughput medical image data mining research like "Radiomics" can influence the cancer research greatly, as it provides a promising non-invasive way of quantifying and monitoring tumor phenotypic characteristics across different cancer types in clinical oncology. 


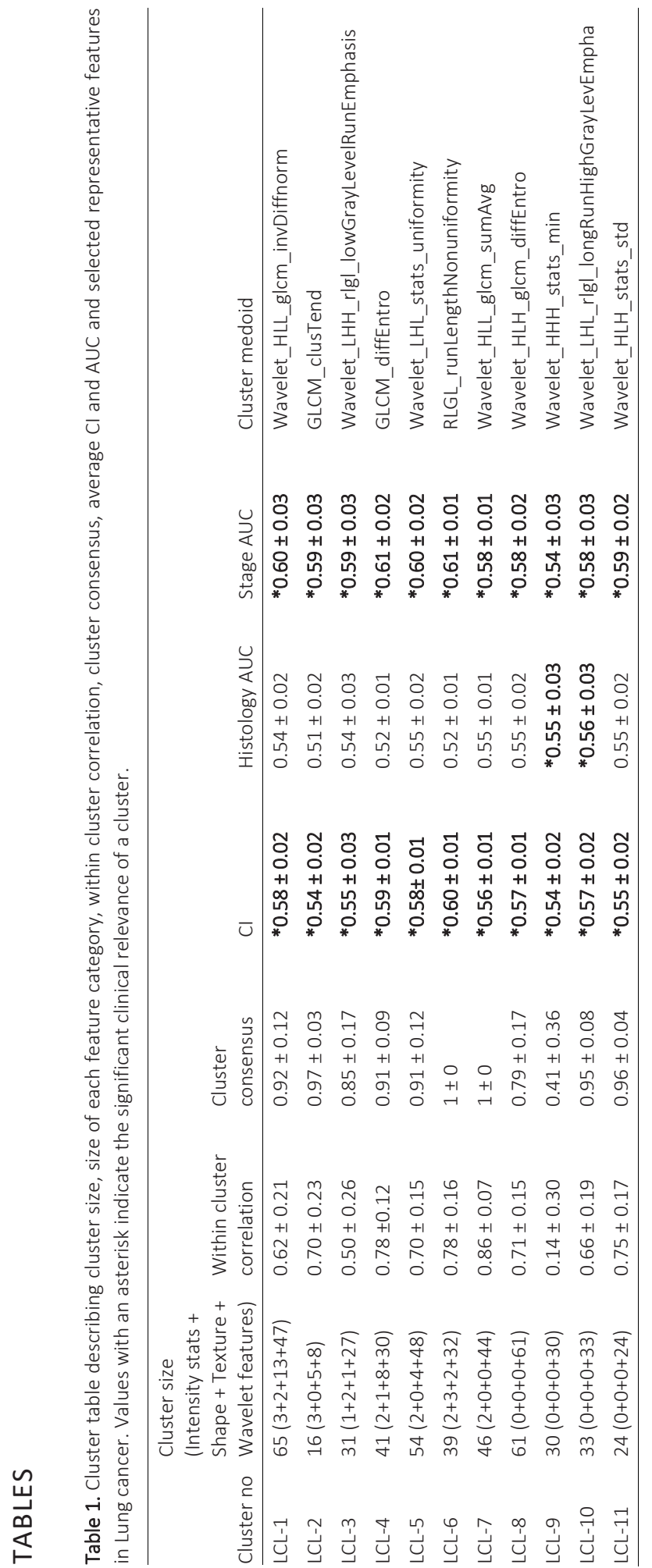




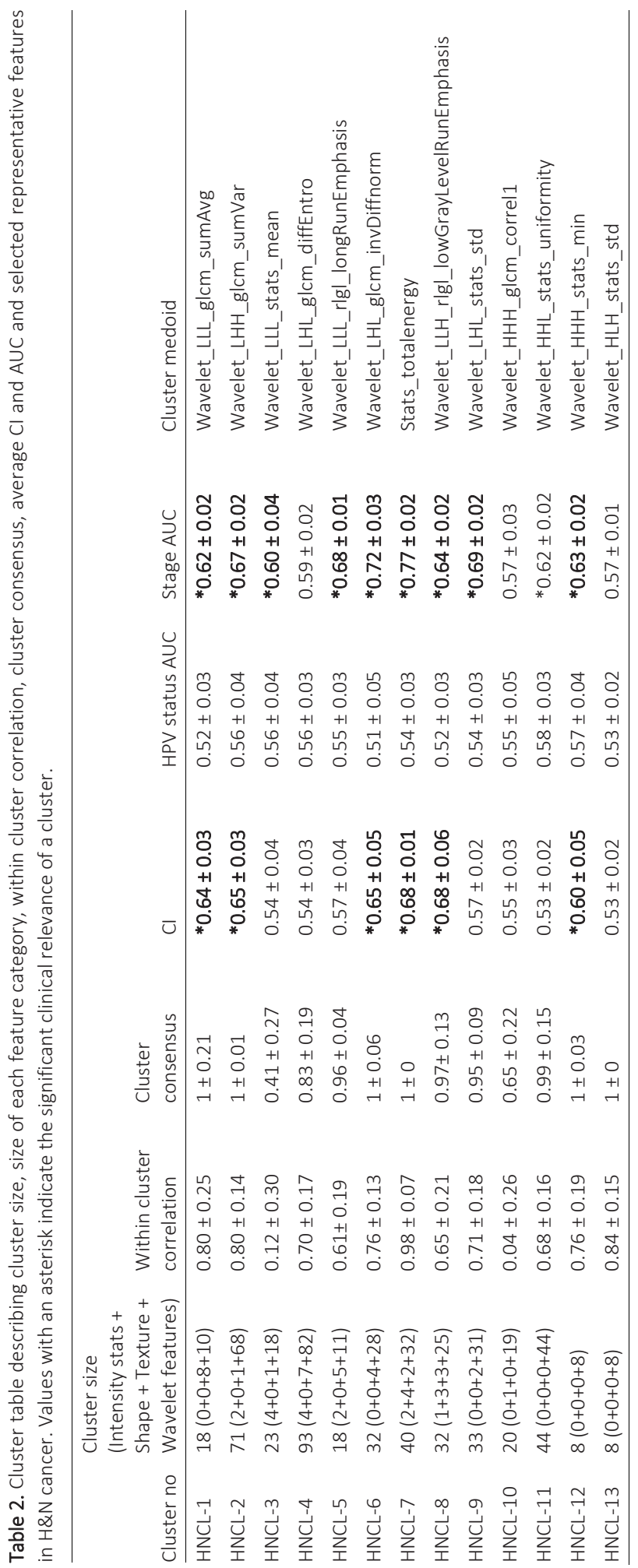




\section{ACKNOWLEDGEMENTS}

Authors also acknowledge financial support from the National Institute of Health $(\mathrm{NIH}$ USA U01 CA 143062-01, and NIH-USA U01 CA 190234-01), EU 7th framework program (EURECA, ARTFORCE), Kankeronderzoekfonds Limburg from the Health Foundation Limburg and the Dutch Cancer Society (KWF UM 2009-4454, KWF MAC 2013-6425).

\section{SUPPLEMENTARY INFORMATION}

http://www.nature.com/articleassets/npg/srep/2015/150605/srep11044/extref/srep11044-s1.pdf 


\section{REFERENCES}

1 Chung, C. H., Levy, S., Chaurand, P. \& Carbone, D. P. Genomics and proteomics: emerging technologies in clinical cancer research. Crit. Rev. Oncol. Hemat. 61, 1-25 (2007).

2 Kurland, B. F. et al. Promise and pitfalls of quantitative imaging in oncology clinical trials. Magn. Reson. Imaging 30, 1301-1312 (2012).

3 Buckler, A. J., Bresolin, L., Dunnick, N. R. \& Sullivan, D. C. Quantitative imaging test approval and biomarker qualification: interrelated but distinct activities. Radiology 259, 875-884 (2011).

4 Buckler, A. J., Bresolin, L., Dunnick, N. R. \& Sullivan, D. C. A collaborative enterprise for multi-stakeholder participation in the advancement of quantitative imaging. Radiology 258, 906-914 (2011).

5 Zhao, B. et al. A pilot study of volume measurement as a method of tumor response evaluation to aid biomarker development. Clin. Cancer Res. 16, 4647-4653 (2010).

6 Birchard, K. R., Hoang, J. K., Herndon, J. E. \& Patz, E. F. Early changes in tumor size in patients treated for advanced stage nonsmall cell lung cancer do not correlate with survival. Cancer 115, 581-586 (2009).

7 Lambin, P. et al. Radiomics: extracting more information from medical images using advanced feature analysis. Eur. J. Cancer 48, 441-446 (2012).

8 Kumar, V. et al. Radiomics: the process and the challenges. Magn. Reson. Imaging 30, 1234-1248 (2012).

9 Lambin, P. et al. Predicting outcomes in radiation oncology-multifactorial decision support systems. Nat. Rev. Clin. Oncol. 10, 27-40 (2012).

10 El Naqa, I. et al. Exploring feature-based approaches in PET images for predicting cancer treatment outcomes. Pattern Recogn. 42, 1162-1171 (2009).

11 Tixier, F. et al. Intra-tumor heterogeneity on baseline 18F-FDG PET images characterized by textural features predicts response to concomitant radio-chemotherapy in eosophageal cancer. J. Nucl. Med. 52, 369-378 (2011).

12 Vaidya, M. et al. Combined PET/CT image characteristics for radiotherapy tumor response in lung cancer. Radiother. Oncol. 102, 239-245 (2012).

13 Ganeshan, B., Panayiotou, E., Burnand, K., Dizdarevic, S. \& Miles, K. Tumour heterogeneity in non-small cell lung carcinoma assessed by CT texture analysis: a potential marker of survival. Eur. Radiol. 22, 796802 (2012).

14 Nicolasjilwan, M. et al. Addition of MR imaging features and genetic biomarkers strengthens glioblastoma survival prediction in TCGA patients. J. Neuroradiol. doi: 10.1016/j.neurad.2014.02.006 (2014)

15 Ganeshan, B., Abaleke, S., Young, R. C., Chatwin, C. R. \& Miles, K. A. Texture analysis of non-small cell lung cancer on unenhanced computed tomography: initial evidence for a relationship with tumour glucose metabolism and stage. Cancer Imaging 10, 137 (2010).

16 Ganeshan, B. et al. Non-small cell lung cancer: histopathologic correlates for texture parameters at CT. Radiology 266, 326-336 (2013).

17 Segal, E. et al. Decoding global gene expression programs in liver cancer by noninvasive imaging. Nat. Biotechnol. 25, 675-680 (2007).

18 Aerts, H. J. et al. Decoding tumour phenotype by noninvasive imaging using a quantitative radiomics approach. Nat. Commun. doi:10.1038/ncomms5006 (2014).

19 Zinn, P. O. et al. Radiogenomic mapping of edema/cellular invasion MRI-phenotypes in glioblastoma multiforme. PLOS ONE 6, e25451 (2011).

20 Monti, S., Tamayo, P., Mesirov, J. \& Golub, T. Consensus clustering: a resampling-based method for class discovery and visualization of gene expression microarray data. Mach. Learn. 52, 91-118 (2003).

21 Haralick, R. M., Shanmugam, K. \& Dinstein, I. H. Textural features for image classification. IEEE Trans. Syst., Man, Cybern., 6, 610-621 (1973).

22 Galloway, M. M. Texture analysis using gray level run lengths. Comput. Vision Graph. 4, 172-179 (1975).

23 Deasy, J. O., Blanco, A. I. \& Clark, V. H. CERR: a computational environment for radiotherapy research. Med. Phys. 30, 979-985 (2003).

24 Harrell, F. Regression modeling strategies: with applications to linear models, logistic regression, and survival analysis, Ch. 10, 247-249 (Springer, 2001). 
25 Schröder, M. S., Culhane, A. C., Quackenbush, J. \& Haibe-Kains, B. survcomp: an R/Bioconductor package for performance assessment and comparison of survival models. Bioinformatics 27, 3206-3208 (2011).

26 Wilkerson, M. D. \& Hayes, D. N. ConsensusClusterPlus: a class discovery tool with confidence assessments and item tracking. Bioinformatics 26, 1572-1573 (2010).

27 Theodoridis, S. \& Koutroumbas, K. Pattern Recognition 4th edn, Ch. 16, 866-872 (Elsevier Science, 2009).

28 Robin, X. et al. pROC: an open-source package for R and $\mathrm{S}+$ to analyze and compare ROC curves. BMC Bioinformatics 12, 77 (2011).

29 Hand, D. J. \& Till, R. J. A simple generalisation of the area under the ROC curve for multiple class classification problems. Mach. Learn. 45, 171-186 (2001).

30 Yee, T. W. The VGAM package for categorical data analysis. J. Stat. Softw. 32, 1-34 (2010).

31 Balagurunathan, Y. et al. Reproducibility and Prognosis of Quantitative Features Extracted from CT Images. Transl. Oncol. 7, 72-87 (2014). 


\section{Chapter}

\section{Exploratory study to identify radiomics classifiers of Lung cancer histology}

Published in: Frontiers in Oncology 6, 71; 2016.

Exploratory study to identify radiomics classifiers of Lung cancer histology Weimiao Wu*, Chintan Parmar*, Patrick Grossmann, John Quackenbush, Philippe Lambin, Johan Bussink, Raymond Mak, Hugo J.W.L. Aerts

*These authors contributed equally to this work 


\section{ABSTRACT}

Background: Radiomics can quantify tumor phenotypic characteristics non-invasively by applying feature algorithms to medical imaging data. In this study of lung cancer patients, we investigated the association between radiomic features and the tumor histologic subtypes (adenocarcinoma and squamous cell carcinoma). Furthermore, in order to predict histologic subtypes, we employed machine-learning methods and independently evaluated their prediction performance.

Methods: Two independent radiomic cohorts with a combined size of 350 patients were included in our analysis. A total of 440 radiomic features were extracted from the segmented tumor volumes of pretreatment $\mathrm{CT}$ images. These radiomic features quantify tumor phenotypic characteristics on medical images using tumor shape and size, intensity statistics and texture. Univariate analysis was performed to assess each feature's association with the histological subtypes. In our multivariate analysis, we investigated 24 feature selection methods and three classification methods for histology prediction. Multivariate models were trained on the training cohort and their performance was evaluated on the independent validation cohort using the area under ROC curve (AUC). Histology was determined from surgical specimen.

Results: In our univariate analysis, we observed that fifty-three radiomic features were significantly associated with tumor histology. In multivariate analysis, feature selection methods Relieff and its variants showed higher prediction accuracy as compared to other methods. We found that Naive Bayes classifier outperforms other classifiers and achieved the highest AUC $\left(0.72 ; p\right.$-value $\left.=2.3 \times 10^{-7}\right)$ with five features: Stats_min, Wavelet_HLL_rlgl_lowGrayLevelRunEmphasis, Wavelet_HHL_stats_median, Wavelet_HLL_stats_skewness, Wavelet_HLH_glcm_clusShade.

Conclusions: Histological subtypes can influence the choice of a treatment/therapy for lung cancer patients. We observed that radiomic features show significant association with the lung tumor histology. Moreover, radiomics based multivariate classifiers were independently validated for the prediction of histological subtypes. Despite achieving lower than optimal prediction accuracy (AUC 0.72), our analysis highlights the impressive potential of non-invasive and cost-effective radiomics for precision medicine. Further research in this direction could lead us to optimal performance and therefore to clinical applicability, which could enhance the efficiency and efficacy of cancer care. 


\section{INTRODUCTION}

Lung cancer is the leading cause of cancer related deaths worldwide with 150,000 deaths per year in United States(1). Lung cancer is clinically divided into two groups: small cell lung cancer (SCLC, 25\%) and non-small cell lung cancer (NSCLC, 75\%)(2). The most common histological subtypes of NSCLC are adenocarcinoma ( 38\%) and squamous cell carcinoma $(\sim 20 \%)(3)$. These two subtypes have distinct histologic features i.e. squamous cell carcinoma are associated with intercellular bridging and individual cell keratinization, whereas glandular architecture is prominent for adenocarcinoma $(3,4)$. Histological classification of lung cancer provides important information about tissue characteristics and anatomical location. Adenocarcinoma often develops at the periphery of the lungs, while squamous carcinoma is generally located more centrally (5). Differences have also been found in expression of glycolysis and hypoxia related markers between histological subtypes, which suggests histology-specific glucose metabolism in $\operatorname{NSCLC}(5,6)$. In addition, histological tumor classification could determine the optimal treatment and/or therapy strategies(7). Recent advancement in the therapy for lung cancer is characterized by discovery of targetable mutations and histology based therapeutic regimen selection(7,8). For example, Pemetrexed is the preferred treatment for stage IV lung adenocarcinoma, whereas bevacizumab is not recommended for squamous carcinoma due to the risk of pulmonary hemorrhage observed in phase II trials (8-10). It has also been shown that treatment for stage III lung cancer, patients with squamous carcinoma has significant improvement in survival with cisplatin/gemcitabine versus cisplatin/pemetrexed, but for adenocarcinoma patients, the latter treatment provides superior survival rate(11,12). More importantly, histology classification increases the likelihood of identifying patients with targetable mutations like EGFR mutations which occurs primarily in adenocarcinoma(4).

In routine clinical practice, the most common way of classifying tumor histology is through the histopathological analysis of tumor tissues via biopsy. This is clinically limited by the inherent risk of invasive procedures as well as poor time and cost efficiency (8). Therefore, automatic, non-invasive, and cost-effective alternatives are desired. Medical imaging provides promising opportunities in this regard. It assesses the tumor tissue characteristics non-invasively. Furthermore, it is relatively cost-effective and is already a routinely used clinical practice for oncologic diagnosis, staging, and treatment guidance(13-15).

Radiomics, a high throughput data mining approach, can exploit the non-invasive medical image data (14). It focuses on extracting a large number of quantitative imaging features, which can provide a detailed and comprehensive characterization of the tumor phenotype (8). Several studies have shown the prognostic/predictive power of radiomic features in different cancer types by using different medical imaging modalities like CT $(16,17), \operatorname{MRI}(18), \operatorname{PET}(15,19,20)$ US $(21)$. It has been shown that radiomic features are associated with several clinically relevant factors, such as tumor stage (22), tumor metabolism(23), overall patient survival(17,24), metastasis (13), treatment re- 
sponse(25), and the underlying gene expression profiles $(26,27)$. These associations can be leveraged to build efficient and effective prediction/prognostic models. Therefore, radiomics is a promising field providing a non-invasive and cost effective way for personalized medicine.

A limited number of studies have investigated the association of radiomic features and NSCLC tumor histology $(22,28)$. Most of them used a clustering based unsupervised approach for associating radiomic features with tumor histological subtypes. However, in order to achieve higher prediction accuracies, supervised methods are generally preferred over unsupervised approaches if labeled data is available. Furthermore, like any other high-throughput data mining approach, radiomics also falls prey to the curse of dimensionality which means we would need to get an enormous amount samples due to high dimensional radiomic features (29). Feature/variable selection is one of the solutions to this problem(30). Many feature selection methods have been proposed in machine learning literature, which should be applied for radiomics based predictive analyses (31).

In this study, we investigated twenty-four commonly used feature selection methods and three supervised machine-learning classification methods for the prediction of lung cancer histologic subtypes, using independent training and validation cohorts from two different institutions. We first reduced the radiomic feature space into a nonredundant subspace by using correlation based feature elimination. Second, we applied twenty-four (Information Gain, Gain Ratio Gini Index, MDL, DKM, ReliefF, and their variants) univariate filter based feature selection methods to the resultant nonredundant feature subset. We chose these filter-based methods because of their popularity in the literature and their high computational efficiency.

The main objective of this study was to investigate the association between radiomic features and lung tumor histology. We employed machine-learning methods to build radiomics based multivariate classifiers for the prediction of tumor histology. Noninvasive and cost-effective radiomic data could improve the histological classification and hence the treatment/therapy, which in general could have a large impact in cancer care. With improving image feature extraction techniques, a higher accuracy in classification is expected to achieve. This work will serve as a promising prognostic tool for informing treatment choice and fostering personalized therapy for lung cancer patients.

\section{METHODS}

\section{Datasets}

We used two NSCLC cohorts collected at different institutions in the Netherlands. The training dataset (Lung1) contains 198 patients (mean age 69.5, range 34-88 years) with pathologically confirmed adenocarcinoma $(n=152)$ or squamous carcinoma $(n=51)$, UICC stages I-IIIb, treated with radical radiotherapy or with chemo-radiation at MAASTRO 
Clinic in Maastricht in the Netherlands. Classification of the tumors as either adenocarcinoma or squamous cell carcinoma was based on H\&E (Haemotoxylin and Eosin) staining according to the World Health Organization (WHO) classification of malignant lung tumors. Experienced radiation oncologists using a standard clinical delineation protocol performed delineation based on fused PET-CT imaging.

The test dataset (Lung2) has 152 patients (mean age 65.6, range 41-86 years) with pathologically confirmed adenocarcinoma $(n=62)$ or squamous carcinoma $(n=90)$, stages (I-IVa), treated at Radboud University Medical Center in Nijmegen, the Netherlands, between February 2004 and October 2011. Histological classification was based on H\&E staining according to the WHO classification of malignant lung tumors. Manual delineations of the CT-scans were available for all included patients in the two datasets. Further details regarding the two data sets can be found in a previous paper (32). The Institutional Review Board of the Maastricht University Medical Center (MUMC+) and the Institutional Review Board of the Radboud University Medical Center (RUMC) waved review due to the retrospective nature of this study (32).

\section{Radiomic features}

Tumor phenotypic characteristics were quantified by extracting 440 3D radiomic features from the segmented tumor regions of pretreatment CT images(32). All radiomic features can be divided into three groups: (1) Intensity: These features quantify the density of the tumor region on the CT image from the first order histogram of voxel intensities. (2) Shape: These features quantify the 3D geometric properties of the tumors. (3) Texture: Textural features quantify the intra-tumor heterogeneity by using the gray level co-occurrence (GLCM) and gray level run length matrices (GLRLM). Intensity and textural features were also computed after applying 3D wavelet transformations (coiflet filters) to the original image. Matlab R2012b was used for the image analysis, and radiomic features were automatically extracted using Matlab R2012b. Details about the image analysis as well as the mathematical definition of the radiomic features can be found in previous literature(32). All radiomic data was centered and scaled before performing the analysis.

\section{Univariate analysis}

The association between the radiomic features and histological subtypes was assessed using the area under the receiver operating characteristic curve (ROC curve) (33). We computed AUC for all the features in a univariate manner. Significance was estimated using a random permutation test with iteration of 1000 . The analysis was performed using R package survcomp(34). 


\section{Feature selection}

Like any other high throughput approach, radiomics also has highly redundant feature space. So if we just rank features based on feature relevance, it is likely that highly correlated features have similar rank and they end up together in the selected feature subset(35). Several studies have discussed this issue with respect to filter based feature selection methods $(36,37)$. Besides, correlated features can cause the classifiers to be sensitive to small changes in the datasets. This could cause a severe problem in cohorts with a different structure of collinearity(38).

To address this problem we used a simple two-stage feature selection method by combing correlation based feature elimination and univariate feature selection. In the first stage, we eliminated highly correlated features using a correlation matrix. We calculated column-wise average absolute correlation $C=\frac{1}{n} \sum_{j} c_{i j}$ for each feature. A threshold $\mathrm{M}$ is set for elimination, that is, for each pair-wise correlation $c_{i j}$ that exceed $\mathrm{M}$, we removed the feature with higher column-wise average absolute correlation $\mathrm{C}$. By eliminating those highly correlated features, we are left with "non-redundant" set of features. This process was implemented by R package 'corrplot' with 'findCorrelation' function (39).

In the second stage, we applied univariate feature selection methods to the nonredundant feature set generated in the first stage, and chose top ranked features. Feature ranking methods that we have used in this study are Gini index, Information Gain, Gain ratio, MDL, DKM and RelifF and their variants $(40,41)$. Detailed description of these methods can be found in documentation of R package "CORElearn"(42).

Most of the used feature selection methods rank features based on their discriminating abilities between classes. Assume we have a set of $m$ dimensional feature vectors $\mathrm{A}=\left(\mathrm{A}_{1}, \mathrm{~A}_{2}, \ldots \mathrm{A}_{\mathrm{m}}\right)$ and $\mathrm{c}$ number of labeled classes $\tau=\left(\tau_{1}, \tau_{2}, \ldots \tau_{c}\right)$. They evaluate each feature by the purity gained by adding the split on $A_{i}=a_{i, j}$. Split is the partitioning of samples according the values of feature at evaluation. $p\left(\tau_{i}\right)$ is the probability of class $\tau_{i}$, and $p\left(\tau_{i} \mid a_{i, j}\right)$ is the probability of class $\tau_{i}$ conditioned on the feature $A_{i}$ has value $a_{i, j}$ (43). These information theory based feature selection approaches and their corresponding scoring functions are described in Table 1 . The derivatives and equations are cited and summarized from $(40,43)$.

\section{ReliefF}

ReliefF (44) evaluates partitioning power of features based on how well their values distinguish between very similar instances. Given a randomly selected instance $R_{i}$, it searches k nearest neighbors from the same class, and calls them "nearest hits" $H$, and also k nearest neighbors from the different class, and called them "nearest miss" M. It then updates the weight $W_{v}$ for all attributes depending on $R_{i}(40)$. The process is repeated for $\mathrm{m}$ times and result is averaged over $\mathrm{m}$ iterations, the function for iteration $\mathrm{v}$ is: 


$$
W_{v}=W_{v}-\frac{1}{m} \operatorname{con}\left(A_{v}, R_{i}, H\right)+\frac{1}{m} \sum_{t=1, t \neq R_{i}}^{c} \frac{p\left(\tau_{t}\right) \operatorname{con}\left(A_{v}, R_{i}, M(C)\right)}{1-p\left(R_{i}, \tau\right)}
$$

Where $\operatorname{con}\left(A_{v}, R_{i}, H\right)$ and $\operatorname{con}\left(A_{v}, R_{i}, M\right)$ are the contribution functions of nearest neighbors (hits and misses). For example, a simple contribution function can be averaging differences of feature's value for $k$ neighbors: $\operatorname{con}\left(A_{v}, R_{i}, H\right)=\frac{1}{k} \sum_{j=1}^{k} \operatorname{diff}\left(A_{v}, R_{i}, H_{j}\right)$. ReliefF can efficiently evaluate features when there are strong dependencies among them (40), but like other feature filtering methods, it could not discriminate between redundant features.

For each feature ranking method, we varied the selection size from five to 45 $(5,10,15, \ldots 45)$ and fit three classifier on the selected feature subset using training dataset. We then evaluate those classifiers by evaluating their prediction performance on validation cohort. Performance of each classifier is measured by AUC. All feature ranking part is performed with R package "CORElearn" in R 3.2.0 (42).

\section{Classifier models}

Three classifiers: Random Forests, Naive Bayesian, and K-Nearest Neighbors were evaluated in this study.

Random Forest is an ensemble learning method for classification, which consists of a collection of decision trees (45). It uses weighted average of those trees for the final decision. This classifier is robust to noises and outliers, and can handle high dimensional spaces fast, but it has been observed to have overfitting problem (45). In this experiment, we set the number of decision trees to 100 , and the number of nearest instances for weighted classification to 30.

Naïve Bayes is another efficient learning algorithms for machine learning. It is a probabilistic classifier based on Bayes's rule and strong conditional independence assumption among features. The probability of an example $E=\left(x_{1}, x_{2} \ldots x_{n}\right)$ belonging to class $c$ is defined as $p(c \mid E)=\frac{p(E \mid c) p(c)}{p(E)}$. E is classified to a positive class if and only if $f_{b}(E)=\frac{p(C=+\mid E)}{p(C=-\mid E)} \geq 1$. Assume all features are independent and given the class $p(E \mid c)=p\left(x_{1}, x_{2}, \ldots x_{n} \mid c\right)=\prod_{i=1}^{n} \frac{p\left(x_{i} \mid C=+\right)}{p\left(x_{i} \mid C=-\right)}$, the Bayes classifier is: $f_{n b}(E)=$ $\frac{p(C=+)}{p(C=-)} \prod_{i=1}^{n} \frac{p\left(x_{i} \mid C=+\right)}{p\left(x_{i} \mid C=-\right)}$.

Naïve Bayes has advantage of requiring small amount of training data to estimate each parameter. Although Naive Bayes has strong independency assumptions which is hardly to meet in practice, it has been shown to be effective even in cases of completely deterministic dependency among features(46).

$\mathrm{K}$-Nearest Neighbors is another non-parametric method used for classification. It is one of the simplest learning methods. The advantage of K-Nearest Neighbors is that it makes no assumption about the characteristic of the features. One major problem is that it is easily misled by irrelevant features to the classification (noise) and highly sus- 
ceptible to curse of dimensionality. Therefore feature selection is important before fitting this model(47). Moreover, it is computationally intensive classification method.. We used $k=9$ for the implementation of this method.

\section{RESULTS}

A total of 440 radiomic features were investigated in terms of their association with and power to predict tumor histology. Feature selection and classification training was done using the training cohort Lung1 $(n=198)$ and the performance was evaluated in the independent validation cohort Lung2 $(n=152)$.

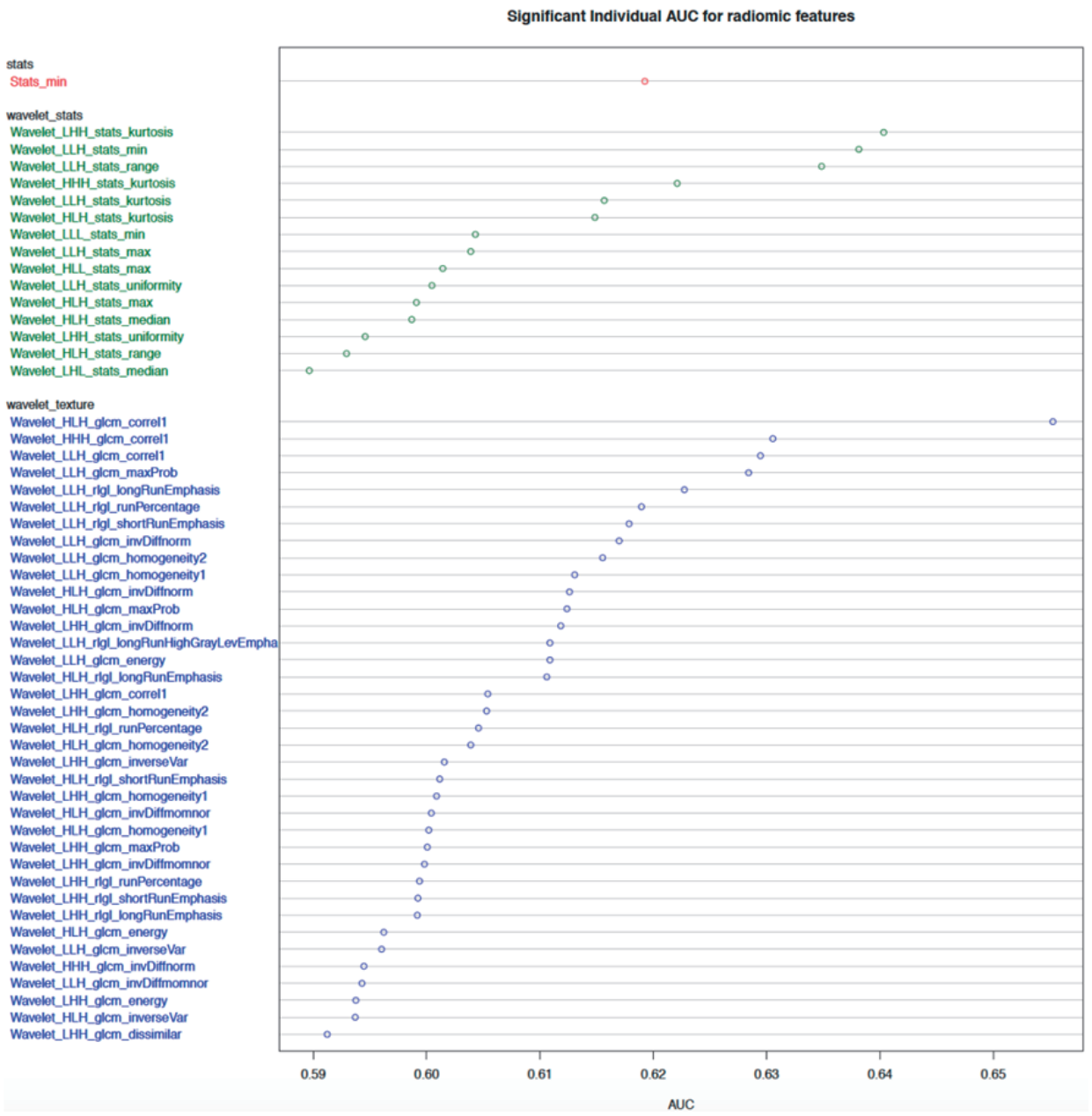

Figure 1. Plot of univariate AUC for fifty-three significant radiomic features 
Univariate analysis of the training dataset shows fifty-three features have significant predictive power (5\% FDR corrected), nearly all of which are wavelet transformed features. Wavelet_HLH_glcm_correl1 had the highest AUC of 0.66 (Cl:0.57-0.74, P value: 0.003) (Figure 1). The adenocarcinoma subgroup has a higher value than the squamous carcinoma subgroup for nine Gray-Level Co-Occurrence Matrix (GLCM) based texture features (HLH and LLH wavelet transformed Energy, Homogeneity1, Homogeneity2, Inverse Variance, LLH wavelet transformed Maximum Probability) and two Gray-Level Run-Length texture features (LLH wavelet transformed Long-Run-Emphasis and Long Run High Gray Level Emphasis). On the other hand, the squamous carcinoma subgroup has a higher value for four RLGL features (HLH and LLH wavelet transformed Run Percentage and Short Run Emphasis) and one statistic feature (LLH wavelet transformed Kurtosis).

In multivariate analysis, we observed that about $75 \%$ of the features had absolute pairwise Pearson correlations higher than 0.8 , and 67\% were over 0.9 (Table 2). To reduce redundancy, we removed features having high absolute pair-wise correlation (C $=0.8$ ). The feature number was reduced from 440 to 67 after eliminating redundant features (Figure 2). The mean of the absolute pair-wise correlations was reduced from 0.36 to 0.18 and the interquartile range (IQR) for the correlations was reduced from 0.39 to 0.20 .

To select the most relevant features, we applied 24 filtering methods to the reduced feature sets. For each filtering method, we incrementally selected 5 to 45 features with an increment of 5 features $(5,10,15, \ldots 45)$. Three classifiers were then developed on selected features in the training dataset and the classification accuracy of each classifier was tested on the validation dataset (Fig 3, Fig 4, Fig 5). The model with the best performance $\left(A \cup C=0.72\right.$, $P$ value $=2.3 \times 10^{-7}$ ) was Naive Bayes, with five predictors selected by ReliefFDistance. We obtained the optimal cutoff on the ROC curve of training cohort and used that cutoff of probability score on validation cohort to measure other prediction measures (Table 2).

As far as feature selection method is concerned, ReliefFDistance showed the highest predictive performance with all three classifiers: Random Forest (AUC $=0.69$ ), Naïve Bayes $(A \cup C=0.72)$, and K-Nearest Neighbors $(A \cup C=0.64)$. Feature selection method ImpurityHellinger for Random forest ( $A \cup C=0.61$ ), Gain Ratio for $K$ Nearest Neighbor ( $A \cup C=0.55)$, and EqualHellinger $(A \cup C=0.62$ ) for Naïve Bayes showed lowest predictive performance. It can be observed that features evaluated using Relieff variants had the most favorable performances for all three classifiers (see Fig3-5).

In order to compare overall performance the classifiers, we used the median AUC across all twenty-four feature selection method as a representative AUC of each classifier. Naïve Bayes had the highest performance while K-Nearest Neighbor showed the lowest performance. Random Forest is the least sensitive to feature selection methods as it showed very little standard deviation in AUC (Table 3). 


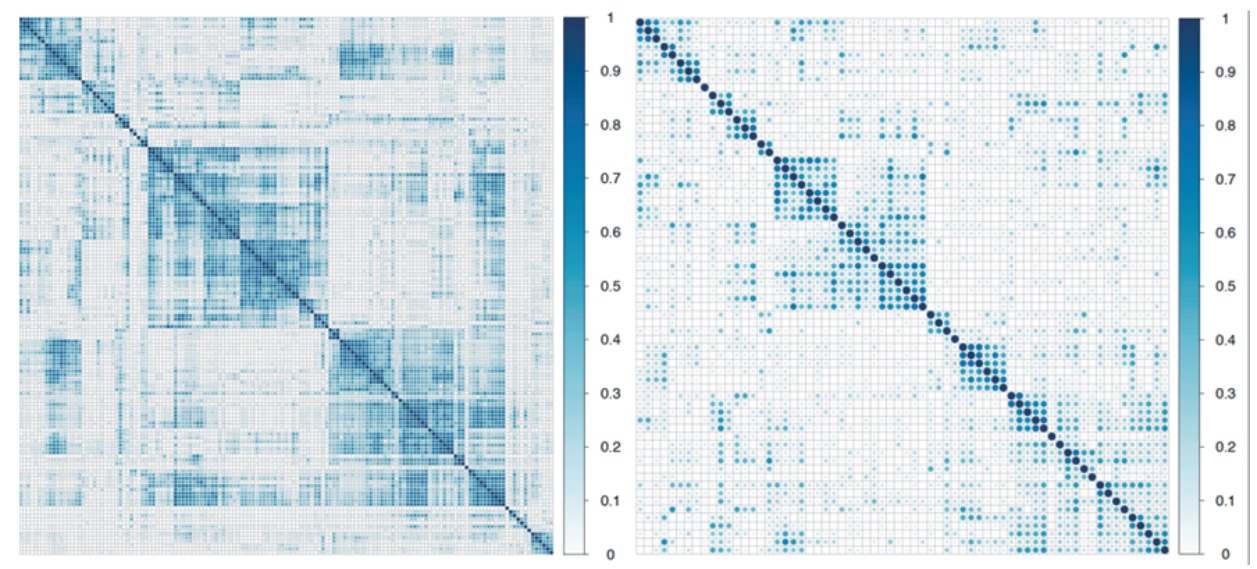

Figure 2. Plot of absolute value of pair-wise Pearson correlations of radiomic features in the training dataset before and after correlation filtering.

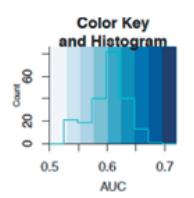

\section{Random Forest Performance}

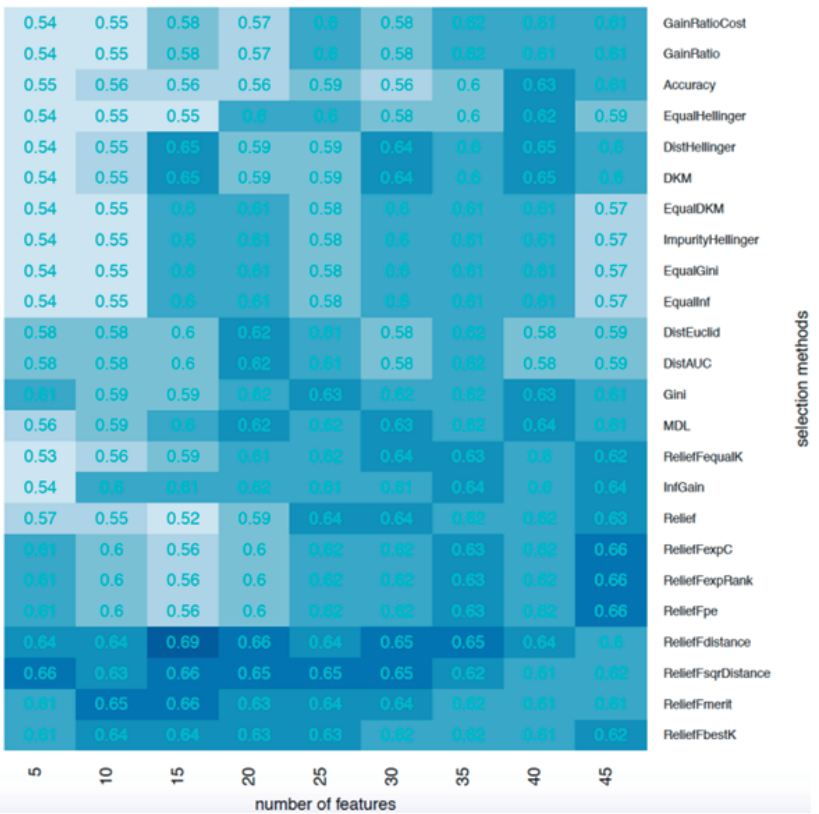

Figure 3. Heatmap describing the predicative performance (AUC) of Random Forest in NSCLC histology classification across feature selection methods (in columns) and range of selection sizes (in rows). 


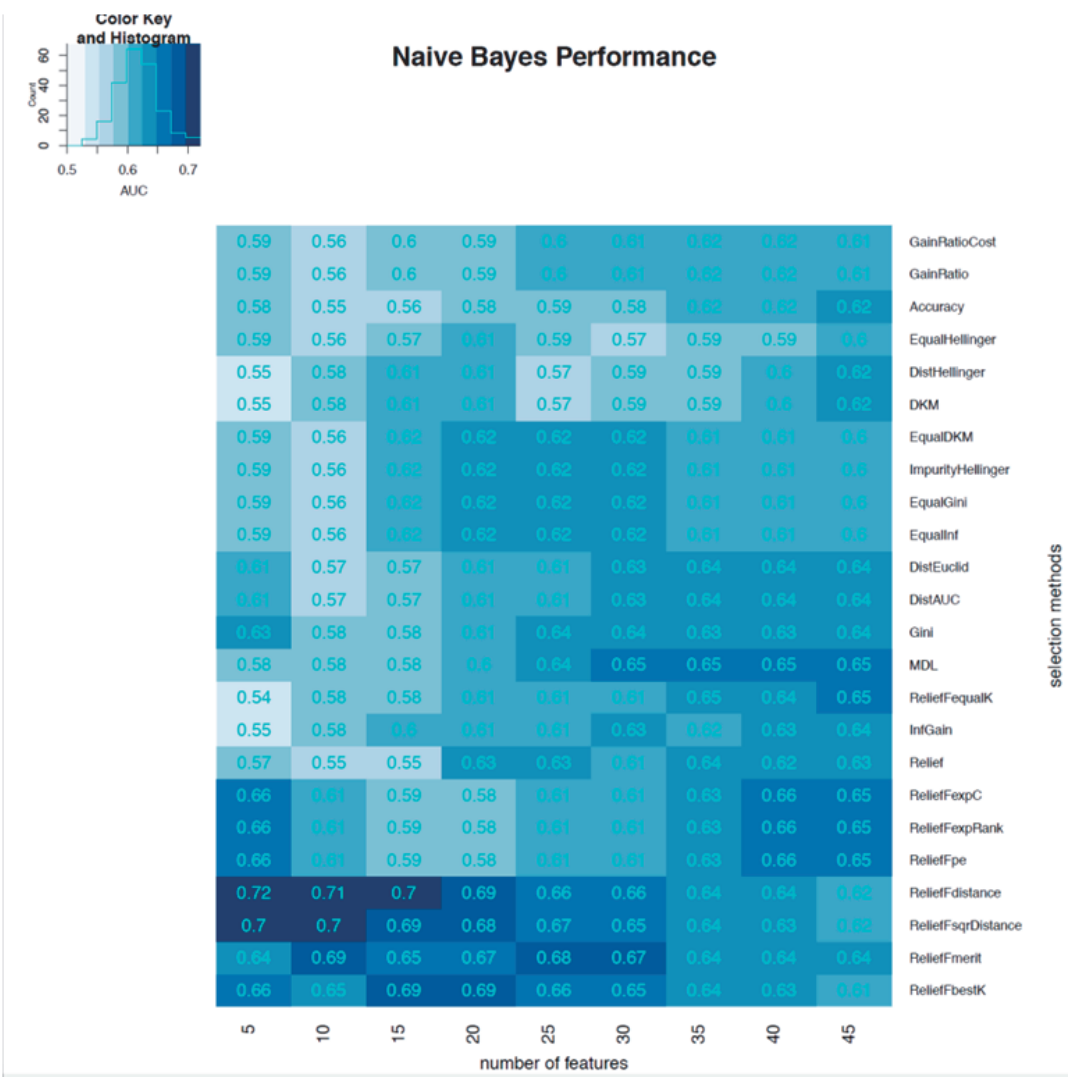

Figure 4. Heatmap describing the predicative performance (AUC) of Naïve Bayes in NSCLC histology classification across feature selection methods (in columns) and range of selection sizes (in rows).

\section{DISCUSSION}

Medical imaging has the capacity to assess tissues characteristics non-invasively and therefore it is routinely used for diagnostic and treatment purposes in cancer care. An emerging field radiomics, quantifies phenotypic characteristic of tumor tissues on medical images. In this study, we investigated the association of radiomic features and the NSCLC histological subtypes. In our univariate analysis we observed fifty-three features having significant association with histological subtypes. Despite the difference in the class distributions between training and validation dataset, the multivariate machine learning models achieved high prediction accuracy, which suggests the effectiveness of these advanced machine-learning approaches as well as the strong association of radiomic features and NSCLC histology. 


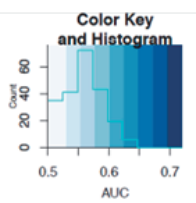

\section{K-Nearest Neighbors Performance}

\begin{tabular}{|c|c|c|c|c|c|c|c|c|c|}
\hline 0.53 & 0.52 & 0.47 & 0.52 & 0.52 & 0.51 & 0.55 & 0.52 & 0.54 & Gainfatiocost \\
\hline 0.53 & 0.52 & 0.47 & 0.52 & 0.52 & 0.51 & 0.55 & 0.52 & 0.54 & GainRatio \\
\hline 0.5 & 0.5 & 0.48 & 0.52 & 0.5 & 0.5 & 0.53 & 0.57 & 0.57 & Accuracy \\
\hline 0.53 & 0.52 & 0.56 & 0.51 & 0.58 & 0.56 & 0.56 & 0.57 & 0.58 & Equalitellinger \\
\hline 0.55 & 0.54 & 0.54 & 0.52 & 0.54 & 0.52 & 0.56 & 0.55 & 0.55 & Disthellinger \\
\hline 0.55 & 0.54 & 0.54 & 0.52 & 0.54 & 0.52 & 0.56 & 0.55 & 0.55 & DKM \\
\hline 0.53 & 0.52 & 0.52 & 0.56 & 0.53 & 0.59 & 0.56 & & 0.57 & EqualoKM \\
\hline 0.53 & 0.52 & 0.52 & 0.56 & 0.53 & 0.59 & 0.56 & & 0.57 & ImpurityHtellinger \\
\hline 0.53 & 0.52 & 0.52 & 0.56 & 0.53 & 0.59 & 0.56 & & 0.57 & Equaligini \\
\hline 0.53 & 0.52 & 0.52 & 0.56 & 0.53 & 0.59 & 0.56 & & 0.57 & Equallnt \\
\hline 0.55 & 0.58 & 0.59 & 0.56 & 0.58 & 0.56 & 0.53 & 0.56 & 0.57 & DistEuclid \\
\hline \multirow[t]{2}{*}{0.55} & 0.58 & 0.59 & 0.56 & 0.58 & 0.56 & 0.53 & 0.56 & 0.57 & Distauc \\
\hline & 0.57 & 0.52 & 0.58 & 0.51 & 0.56 & 0.59 & 0.58 & 0.57 & Gini \\
\hline 0.55 & 0.57 & 0.52 & & 0.51 & 0.55 & 0.55 & 0.56 & 0.6 & MDL. \\
\hline 0.52 & 0.58 & 0.48 & 0.55 & 0.52 & 0.55 & 0.53 & 0.56 & 0.56 & Relief Fequalk \\
\hline 0.55 & & 0.56 & 0.51 & 0.55 & 0.56 & 0.58 & 0.56 & 0.59 & IntGain \\
\hline 0.58 & 0.58 & 0.55 & 0.56 & 0.51 & 0.53 & 0.56 & 0.53 & 0.55 & Rellet \\
\hline 0.57 & 0.59 & 0.59 & 0.55 & 0.57 & 0.57 & 0.57 & 0.57 & 0.6 & RelleffexpC \\
\hline 0.57 & 0.59 & 0.59 & 0.55 & 0.57 & 0.57 & 0.57 & 0.57 & 0.6 & ReliefFexp Rank \\
\hline 0.57 & 0.59 & 0.59 & 0.55 & 0.57 & 0.57 & 0.57 & 0.57 & 0.6 & Rellieffpe \\
\hline 0.64 & 0.59 & 0.58 & 0.58 & & & 0.57 & & & Relieffodistance \\
\hline 0.64 & 0.56 & 0.58 & 0.64 & & 0.58 & & 0.59 & & Relleffsqrostance \\
\hline 0.57 & & & 0.62 & 0.6 & & & 0.63 & 0.63 & RelieffFertt \\
\hline 0.57 & 0.55 & 0.53 & 0.6 & & 0.56 & 0.58 & 0.56 & 0.58 & ReliefFbestK \\
\hline n & 우 & 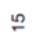 & ని & $\stackrel{\sim}{\sim}$ & 이 & $\ddot{e}$ & o & \& & \\
\hline
\end{tabular}

Figure 5. Heatmap describing the predicative performance (AUC) of K-Nearest Neighbors in NSCLC histology classification across feature selection methods (in columns) and range of selection sizes (in rows).

Our study showed that ReliefF and its variants were optimal among the twenty-four feature selection methods assessed. Particularly, RelifFbestK, ReliefFmerit, ReliefFdistance and ReliefFsqrDistance were consistently effective for all three classifiers. One reason for this is that the Relieff-family does not assume the independence of features, unlike many other feature selection methods. The Relieff algorithms are able to detect context information among features and thus more accurately deals with situations where dependencies are present (48). However, like other feature selection methods, Relieff is also unable to detect redundant features which tend to have similar scores for evaluation(49). We took care of this problem by performing correlation based feature elimination before feature selection stage.

For the performance of three classifiers, Naïve Bayes performs better than Random Forest and K-Nearest Neighbors. Although the median performance across feature selection methods was about the same for Naïve Bayes and Random Forest, the best model achieved by Naïve Bayes (AUC: 0.72; P value $=2.3 \times 10^{-7}$ ) was higher than Random Forest (AUC: 0.68; $\mathrm{P}$ value $=1.38 \times 10^{-5}$ ). K-Nearest Neighbors has the lowest 
performance among the three classifiers, and Relieff could only slightly improve its accuracy. This may because K-Nearest Neighbors is very sensitive to noise (47).

A shortcoming of our study is that the cut-off $M=0.8$ is arbitrarily chosen in the correlation elimination part. One could set a more stringent or relaxed threshold based on the degree of redundancy in the dataset. A better approach would use a range of thresholds, combine them with second stage feature selection, and choose one with the most favorable result. Additionally, this two-stage feature selection is expected to fail when the interaction of two non-informative features has strong predictive power. In this case, a multivariate feature selection method like mRMR is more suitable(35).

Recently, Parmar et al. (22) identified and validated cancer specific radiomic feature clusters using consensus clustering, which provided an important tool to enhance the feature selection process. Their study found radiomic features' association with histology using an unsupervised method and achieved $A \cup C=0.64$ for prediction. In our study by using supervised feature selection methods, we achieved higher AUCs. In another study, Basu et al. used decision trees and support vector machines for tumor classification, and results showed that ReliefF outperformed wrapper methods for $2 \mathrm{D}$ radiomic features(28). However, unlike our study, their results were based on a smaller cohort $(n=74)$ and lacked independent validation due to the unavailability of an independent validation cohort. Lastly Yi Zhang et al presented a two-stage feature selection method by combining ReliefF and mRMR (31). They showed that such a hybrid feature selection approach could improve the effectiveness of gene selection, and this could provide better discrimination for biological subtypes. This new algorithm's ability in selecting radiomic features for histology classification should be evaluated in further studies.

It should also be noted that retrospective cohorts based radiomic studies like this, face many challenges. Radiomic features are sensitive to the variability of imaging scanners and scanning parameters $(50,51)$, tumor delineation methods $(52,53)$, image reconstruction (54) and discretization techniques (55)etc. These different factors are not controlled for retrospective cohorts, which maybe one of the reason for not so impressive performance. Prospective cohorts created by controlling these factors could provide higher performance. In future studies, the performance of classifiers can be enhanced if we incorporate genetic signatures and clinical features like tumor grade, location, smoking history, and obesity $(56,57)$. For example, a recent study showed that body mass index was inversely associated with squamous carcinoma, yet for adenocarcinoma the association was positive(57). It is also important to take genetic heterogeneity into account. Recent studies examining gene expression profiles have identified several genes distinguishing adenocarcinoma and squamous carcinoma (58-60). Hence, future study incorporating clinic characteristics and genomic data will improve classification accuracy.

In conclusion, radiomic features have strong predictive power for classification of NSCLC histology. With expanding cohorts and improving image feature extraction techniques, we expect higher accuracy in classification using radiomics. This work will serve 
as a promising diagnostic tool for identifying lung cancer histology in a non-invasive way and thus informing treatment choices and personalized therapy for lung cancer patients.

\section{TABLES}

Table 1. Feature filtering methods and corresponding scoring schemes.

\begin{tabular}{|c|c|}
\hline $\begin{array}{l}\text { Feature } \\
\text { Filtering } \\
\text { Methods }\end{array}$ & Scoring Function \\
\hline $\begin{array}{l}\text { Information } \\
\text { Gain(44) }\end{array}$ & $I N\left(A_{i}\right)=\sum_{i=1}^{c} p\left(\tau_{i}\right) \log p\left(\tau_{i}\right)-\sum_{j=1}^{a_{m i}} \sum_{i=1}^{c} p\left(\tau_{i} \mid a_{i, j}\right) \log p\left(\tau_{i} \mid a_{i, j}\right)$ \\
\hline Gain Ratio(61) & $G R\left(A_{i}\right)=\frac{\sum_{i=1}^{c} p\left(\tau_{i}\right) \log p\left(\tau_{i}\right)-\sum_{j=1}^{a_{m i}} \sum_{i=1}^{c} p\left(\tau_{i} \mid a_{i, j}\right) \log p\left(\tau_{i} \mid a_{i, j}\right)}{\sum_{j=1}^{a_{m i}} p\left(a_{i j}\right) \log p\left(a_{i j}\right)}$ \\
\hline Gini Index(62) & $G I\left(A_{i}\right)=\sum_{i=1}^{c} p^{2}\left(\tau_{i}\right)-\sum_{j=1}^{a_{m i}} \sum_{i=1}^{c} p^{2}\left(\tau_{i} \mid a_{i, j}\right)$ \\
\hline $\operatorname{MDL}(63)$ & $\begin{array}{l}\quad \operatorname{MDL}\left(A_{i}\right)=\frac{1}{n}\left(\log _{2}\left(\begin{array}{c}n \\
n_{1,}, \ldots, n_{c}\end{array}\right)-\sum_{j=1}^{a_{m i}}\left(\begin{array}{c}n_{. j} \\
n_{1 j}, \ldots n_{c j}\end{array}\right)+\log _{2}\left(\begin{array}{c}n+c+1 \\
c-1\end{array}\right)-\sum_{j=1}^{a_{m i}}\left(\begin{array}{c}n_{. j}+c-1 \\
c-1\end{array}\right)\right. \\
\text { Where } n_{i j} \text { is number of samples from class i with jth value of features. }\end{array}$ \\
\hline $\operatorname{DKM}(40)$ & $\begin{aligned} \operatorname{DKM}\left(A_{i}\right) & =\sum_{i=1}^{c} 2 \sqrt{p\left(\tau_{\max }\right)\left(1-p\left(\tau_{\max }\right)\right.}-\sum_{j=1}^{a_{\operatorname{mi}}} \sum_{i=1}^{c} \sqrt{p\left(\tau_{\max } \mid a_{i j}\right)\left(1-p\left(\tau_{\max } \mid a_{i j}\right)\right.} \\
\text { where } p\left(\tau_{\max }\right) & =\max _{i=1 \text { to } c} p\left(\tau_{i}\right) \text { represent most probable class value. }\end{aligned}$ \\
\hline
\end{tabular}

Table 2. Classification Accuracy of the optimal classifier

\begin{tabular}{llllll}
\hline Optimal Cut-off & Sensitivity & Specificity & PPV & NPV & Accuracy \\
\hline 0.271 & 0.55 & 0.8 & 0.72 & 0.65 & 0.70 \\
\hline
\end{tabular}

Table 3. Table describing the median value of AUC, the optimal number of features, and AUC for best/worst features selection methods.

\begin{tabular}{llll}
\hline Classifier & $\begin{array}{l}\text { AUC } \\
\text { (median } \pm \mathrm{sd})\end{array}$ & $\begin{array}{l}\text { Optimal feature } \\
\text { number }\end{array}$ & Best/worst feature selection method(AUC) \\
\hline Naïve Bayes & $\begin{array}{l}0.64 \pm 0.028 \\
\text { Random Forest }\end{array}$ & 5 & ReliefFdistance(0.72)/ EqualHellinger (0.62) \\
& $0.63 \pm 0.012$ & 15 & $\begin{array}{l}\text { ReliefFdistance }(0.69) / \\
\text { ImpurityHellinger(0.61) }\end{array}$ \\
K-Nearest Neighbor & $0.60 \pm 0.23$ & 20 & ReliefFdstance(0.64)/Gain Ratio(0.55) \\
\hline
\end{tabular}

\section{ACKNOWLEDGEMENT}

Authors acknowledge financial support from the National Institute of Health (NIH-USA U24CA194354 and NIH-USA U01CA190234). This research is also supported by the Dutch technology Foundation STW (grant n 10696 DuCAT). Authors also acknowledge financial support from EU 7th framework program (ARTFORCE - $n^{\circ} 257144$, REQUITE $n^{\circ}$ 601826), SME Phase 2 (EU proposal 673780 - RAIL), the European Program H2020PHC-2015 (BD2decide, n²10274050), Alpe d'HuZes-KWF (DESIGN). 


\section{REFERENCES}

1. Bhattacharjee A, Richards WG, Staunton J, Li C, Monti S, Vasa P, Ladd C, Beheshti J, Bueno R, Gillette M, et al. Classification of human lung carcinomas by mRNA expression profiling reveals distinct adenocarcinoma subclasses. Proc Natl Acad Sci USA (2001) 98:13790-13795. doi:10.1073/pnas.191502998

2. Risch A, Plass C. Lung cancer epigenetics and genetics. International Journal of Cancer (2008) 123:1-7. doi:10.1002/ijc.23605

3. Travis WD. Pathology of lung cancer. Clin Chest Med (2011) 32:669-692. doi:10.1016/j.ccm.2011.08.005

4. Rekhtman N, Ang DC, Sima CS, Travis WD, Moreira AL. Immunohistochemical algorithm for differentiation of lung adenocarcinoma and squamous cell carcinoma based on large series of whole-tissue sections with validation in small specimens. Mod Pathol (2011) 24:1348-1359. doi:10.1038/modpathol.2011.92

5. Schuurbiers OCJ, Meijer TWH, Kaanders JHAM, Looijen-Salamon MG, de Geus-Oei L-F, van der Drift MA, van der Heijden EHFM, Oyen WJ, Visser EP, Span PN, et al. Glucose metabolism in NSCLC is histologyspecific and diverges the prognostic potential of 18FDG-PET for adenocarcinoma and squamous cell carcinoma. J Thorac Oncol (2014) 9:1485-1493. doi:10.1097/JTO.0000000000000286

6. Meijer TWH, Schuurbiers OCJ, Kaanders JHAM, Looijen-Salamon MG, de Geus-Oei L-F, Verhagen AFTM, Lok J, van der Heijden HFM, Rademakers SE, Span PN, et al. Differences in metabolism between adenoand squamous cell non-small cell lung carcinomas: spatial distribution and prognostic value of GLUT1 and MCT4. Lung Cancer (2012) 76:316-323. doi:10.1016/j.lungcan.2011.11.006

7. Manegold C. Treatment algorithm in 2014 for advanced non-small cell lung cancer: therapy selection by tumour histology and molecular biology. Adv Med Sci (2014) 59:308-313. doi:10.1016/ j.advms.2014.08.008

8. Cufer T, Ovcaricek T, O'Brien MER. Systemic therapy of advanced non-small cell lung cancer: majordevelopments of the last 5-years. Eur J Cancer (2013) 49:1216-1225. doi:10.1016/j.ejca.2012.11.021

9. Travis WD. Classification of Lung Cancer. Seminars in Roentgenology (2011) 46:178-186. doi:10.1053/j.ro.2011.02.003

10. Barash O, Peled N, Tisch U, Bunn PA, Hirsch FR, Haick H. Classification of lung cancer histology by gold nanoparticle sensors. Nanomedicine (2012) 8:580-589. doi:10.1016/j.nano.2011.10.001

11. Scagliotti GV, Parikh P, Pawel von J, Biesma B, Vansteenkiste J, Manegold C, Serwatowski P, Gatzemeier $U$, Digumarti R, Zukin M, et al. Phase III study comparing cisplatin plus gemcitabine with cisplatin plus pemetrexed in chemotherapy-naive patients with advanced-stage non-small-cell lung cancer. J Clin Oncol (2008) 26:3543-3551. doi:10.1200/JCO.2007.15.0375

12. Scagliotti G, Hanna N, Fossella F, Sugarman K, Blatter J, Peterson P, Simms L, Shepherd FA. The differential efficacy of pemetrexed according to NSCLC histology: a review of two Phase III studies. Oncologist (2009) 14:253-263. doi:10.1634/theoncologist.2008-0232

13. Coroller TP, Grossmann P, Hou Y, Rios Velazquez E, Leijenaar RTH, Hermann G, Lambin P, Haibe-Kains B, Mak RH, Aerts HJWL. CT-based radiomic signature predicts distant metastasis in lung adenocarcinoma. Radiother Oncol (2015) 114:345-350. doi:10.1016/j.radonc.2015.02.015

14. Lambin P, Rios Velazquez E, Leijenaar R, Carvalho S, van Stiphout RGPM, Granton P, Zegers CML, Gillies R, Boellard R, Dekker A, et al. Radiomics: extracting more information from medical images using advanced feature analysis. Eur J Cancer (2012) 48:441-446. doi:10.1016/j.ejca.2011.11.036

15. Lambin P, van Stiphout RGPM, Starmans MHW, Rios Velazquez E, Nalbantov G, Aerts HJWL, Roelofs E, van Elmpt W, Boutros PC, Granone P, et al. Predicting outcomes in radiation oncology|[mdash]|multifactorial decision support systems. Nat Rev Clin Oncol (2013) 10:27-40. doi:10.1038/nrclinonc.2012.196

16. Hayano K, Yoshida H, Zhu AX, Sahani DV. Fractal analysis of contrast-enhanced CT images to predict survival of patients with hepatocellular carcinoma treated with sunitinib. Dig Dis Sci (2014) 59:19962003. doi:10.1007/s10620-014-3064-z

17. Ganeshan B, Panayiotou E, Burnand K, Dizdarevic S, Miles K. Tumour heterogeneity in non-small cell lung carcinoma assessed by CT texture analysis: a potential marker of survival. Eur Radiol (2012) 22:796-802. doi:10.1007/s00330-011-2319-8 
18. Tiwari P, Kurhanewicz J, Madabhushi A. Multi-kernel graph embedding for detection, Gleason grading of prostate cancer via MRI/MRS. Med Image Anal (2013) 17:219-235. doi:10.1016/j.media.2012.10.004

19. Chen HH, Su W, Hsueh W, Wu Y, Lin F. Summation of F18-FDG Uptakes on PET/CT Images Predicts Disease Progression in Non-small Cell Lung Cancer. International Journal of ... (2010).

20. Nair VS, Gevaert O, Davidzon G, Napel S, Graves EE, Hoang CD, Shrager JB, Quon A, Rubin DL, Plevritis SK. Prognostic PET 18F-FDG uptake imaging features are associated with major oncogenomic alterations in patients with resected non-small cell lung cancer. Cancer Res (2012) 72:3725-3734. doi:10.1158/00085472.CAN-11-3943

21. Fleshman JW, Myerson RJ, Fry RD, Kodner IJ. Accuracy of transrectal ultrasound in predicting pathologic stage of rectal cancer before and after preoperative radiation therapy. Dis Colon Rectum (1992) 35:823-829.

22. Parmar C, Leijenaar RTH, Grossmann P, Velazquez ER, Bussink J, Rietveld D, Rietbergen MM, Haibe-Kains B, Lambin P, Aerts HJWL. Radiomic feature clusters and Prognostic Signatures specific for Lung and Head \& Neck cancer. Scientific Reports (2015) 5:11044. doi:10.1038/srep11044

23. Ganeshan B, Abaleke S, Young RCD, Chatwin CR, Miles KA. Texture analysis of non-small cell lung cancer on unenhanced computed tomography: initial evidence for a relationship with tumour glucose metabolism and stage. Cancer Imaging (2010) 10:137-143. doi:10.1102/1470-7330.2010.0021

24. Hall WA, Djalilian HR, Sperduto PW, Cho KH, Gerbi BJ, Gibbons JP, Rohr M, Clark HB. Stereotactic radiosurgery for recurrent malignant gliomas. JCO (1995) 13:1642-1648.

25. Vaidya M, Creach KM, Frye J, Dehdashti F, Bradley JD, Naqa EI I. Combined PET/CT image characteristics for radiotherapy tumor response in lung cancer. Radiother Oncol (2012) 102:239-245. doi:10.1016/j.radonc.2011.10.014

26. Segal E, Sirlin CB, Ooi C, Adler AS, Gollub J, Chen X, Chan BK, Matcuk GR, Barry CT, Chang HY, et al. Decoding global gene expression programs in liver cancer by noninvasive imaging. Nat Biotechnol (2007) 25:675-680. doi:10.1038/nbt1306

27. Zinn PO, Mahajan B, Majadan B, Sathyan P, Singh SK, Majumder S, Jolesz FA, Colen RR. Radiogenomic mapping of edema/cellular invasion MRI-phenotypes in glioblastoma multiforme. PLoS ONE (2011) 6:e25451. doi:10.1371/journal.pone.0025451

28. Basu S, Hall LO, Goldgof DB, Gu Y, Kumar V, Choi J, Gillies RJ, Gatenby RA. Developing a classifier model for lung tumors in CT-scan images. (2011)1306-1312. doi:10.1109/ICSMC.2011.6083840

29. Friedman JH. On Bias, Variance, 0/1-Loss, and the Curse-of-Dimensionality. Data Mining and Knowledge Discovery (1997) 1:55-77. doi:10.1023/A:1009778005914

30. Guyon I, Elisseeff A. An introduction to variable and feature selection. The Journal of Machine Learning Research (2003) 3:1157-1182.

31. Zhang Y, Ding C, Li T. Gene selection algorithm by combining reliefF and mRMR. BMC Genomics (2008) 9 Suppl 2:S27. doi:10.1186/1471-2164-9-S2-S27

32. Aerts HJWL, Velazquez ER, Leijenaar RTH, Parmar C, Grossmann P, Carvalho S, Cavalho S, Bussink J, Monshouwer R, Haibe-Kains B, et al. Decoding tumour phenotype by noninvasive imaging using a quantitative radiomics approach. Nat Commun (2014) 5:4006. doi:10.1038/ncomms5006

33. Hanley JA, McNeil BJ. The meaning and use of the area under a receiver operating characteristic (ROC) curve. Radiology (1982) 143:29-36. doi:10.1148/radiology.143.1.7063747

34. Schröder MS, Culhane AC, Quackenbush J, Haibe-Kains B. survcomp: an R/Bioconductor package for performance assessment and comparison of survival models. Bioinformatics (2011) 27:3206-3208. doi:10.1093/bioinformatics/btr511

35. Ding C, PENG H. Minimum redundancy feature selection from microarray gene expression data. Journal of Bioinformatics and Computational Biology (2005) 3:185-205.

36. Li W, Yang Y. "How Many Genes are Needed for a Discriminant Microarray Data Analysis," in Methods of Microarray Data Analysis (Boston, MA: Springer US), 137-149. doi:10.1007/978-1-4615-0873-1_11

37. Xiong M, Fang X, Zhao J. Biomarker identification by feature wrappers. Genome Res (2001) 11:18781887. doi:10.1101/gr.190001

38. Dormann CF, Elith J, Bacher S, Buchmann C, Carl G, Carré G, Marquéz JRG, Gruber B, Lafourcade B, Leitão PJ, et al. Collinearity: a review of methods to deal with it and a simulation study evaluating their performance. Ecography (2013) 36:27-46. doi:10.1111/j.1600-0587.2012.07348.x

39. Wei T, Wei MT. Package "corrplot." Statistician (2015) 
40. Robnik-Šikonja M, Kononenko I. Theoretical and Empirical Analysis of ReliefF and RReliefF. Machine Learning (2003) 53:23-69. doi:10.1023/A:1025667309714

41. Dhillon PS, Foster D, Ungar LH. Minimum Description Length Penalization for Group and Multi-Task Sparse Learning. The Journal of Machine Learning Research (2011) 12:525-564.

42. Robnik-Šikonja M, Savicky P. CORElearn-package. rdocumentationorg

43. Robnik-Šikonja M. "Experiments with Cost-Sensitive Feature Evaluation," in Machine Learning: ECML 2003 Lecture Notes in Computer Science. (Berlin, Heidelberg: Springer Berlin Heidelberg), 325-336. doi:10.1007/978-3-540-39857-8_30

44. Kononenko I. "Estimating attributes: Analysis and extensions of RELIEF," in Machine Learning: ECML-94 Lecture Notes in Computer Science. (Berlin, Heidelberg: Springer Berlin Heidelberg), 171-182. doi:10.1007/3-540-57868-4_57

45. Breiman L. Random Forests. Machine Learning (2001) 45:5-32. doi:10.1023/A:1010933404324

46. Rish I. An empirical study of the naive Bayes classifier. in (IBM New York), 41-46.

47. Cunningham P, Delany SJ. k-Nearest neighbour classifiers. Multiple Classifier Systems (2007)

48. Kira K, Rendell LA. The feature selection problem: Traditional methods and a new algorithm. AAAI (1992)

49. Wu T, Xie K, Nie C, Song G. "An Adaption of Relief for Redundant Feature Elimination," in Advances in Neural Networks - ISNN 2012 Lecture Notes in Computer Science. (Berlin, Heidelberg: Springer Berlin Heidelberg), 73-81. doi:10.1007/978-3-642-31362-2_9

50. Mattonen SA, Huang K, Ward AD, Senan S, Palma DA. New techniques for assessing response after hypofractionated radiotherapy for lung cancer. J Thorac Dis (2014) 6:375-386. doi:10.3978/j.issn.20721439.2013.11.09

51. Alobaidli S, McQuaid S, South C, Prakash V, Evans $P$, Nisbet A. The role of texture analysis in imaging as an outcome predictor and potential tool in radiotherapy treatment planning. $\mathrm{Br} J$ Radiol (2014) 87:20140369. doi:10.1259/bjr.20140369

52. Mackin D, Fave X, Zhang L, Fried D, Yang J, Taylor B, Rodriguez-Rivera E, Dodge C, Jones AK, Court L. Measuring Computed Tomography Scanner Variability of Radiomics Features. Invest Radiol (2015) 50:757-765. doi:10.1097/RLI.0000000000000180

53. Zhao B, Tan Y, Tsai WY, Schwartz LH, Lu L. Exploring Variability in CT Characterization of Tumors: A Preliminary Phantom Study. Transl Oncol (2014) 7:88-93. doi:10.1118/1.4793409

54. Galavis PE, Hollensen C, Jallow N, Paliwal B, Jeraj R. Variability of textural features in FDG PET images due to different acquisition modes and reconstruction parameters. Acta Oncol (2010) 49:1012-1016. doi:10.3109/0284186X.2010.498437

55. Leijenaar RTH, Nalbantov G, Carvalho S, van Elmpt WJC, Troost EGC, Boellaard R, Aerts HJWL, Gillies RJ, Lambin P. The effect of SUV discretization in quantitative FDG-PET Radiomics: the need for standardized methodology in tumor texture analysis. Scientific Reports (2015) 5:11075. doi:10.1038/srep11075

56. Bufill JA. Colorectal Cancer: Evidence for Distinct Genetic Categories Based on Proximal or Distal Tumor Location. Ann Intern Med (1990) 113:779-788. doi:10.7326/0003-4819-113-10-779

57. Zein El M, Parent ME, Nicolau B, Koushik A, Siemiatycki J, Rousseau MC. Body mass index, lifetime smoking intensity and lung cancer risk. International Journal of Cancer (2013) 133:1721-1731. doi:10.1002/ijc.28185

58. Boelens MC, van den Berg A, Vogelzang I, Wesseling J, Postma DS, Timens W, Groen HJM. Differential expression and distribution of epithelial adhesion molecules in non-small cell lung cancer and normal bronchus. J Clin Pathol (2007) 60:608-614. doi:10.1136/jcp.2005.031443

59. Kettunen E, Anttila S, Seppänen JK, Karjalainen A, Edgren H, Lindström I, Salovaara R, Nissén A-M, Salo J, Mattson $\mathrm{K}$, et al. Differentially expressed genes in nonsmall cell lung cancer: expression profiling of cancer-related genes in squamous cell lung cancer. Cancer Genet Cytogenet (2004) 149:98-106. doi:10.1016/S0165-4608(03)00300-5

60. Pietanza MC, Ladanyi M. Bringing the genomic landscape of small-cell lung cancer into focus. Nat Genet (2012) 44:1074-1075. doi:10.1038/ng.2415

61. Quinlan JR. C4.5. Elsevier (2014).

62. Breiman L, Friedman JH, Olshen RA, Stone CJ. Classification and regression trees. Wadsworth. Belmont (1984).

63. Kononenko I. On biases in estimating multi-valued attributes. IJCAI (1995) 



\section{Chapter}

\section{Machine learning methods for quantitative radiomic biomarkers}

Published in: Nature Scientific Reports 5, 13087; 2015.

Machine learning methods for quantitative radiomic biomarkers

Chintan Parmar*, Patrick Grossmann*, Johan Bussink, Philippe Lambin, Hugo J.W.L. Aerts

*These authors contributed equally to this work 


\section{ABSTRACT}

Radiomics extracts and mines large number of medical imaging features quantifying tumor phenotypic characteristics. Highly accurate and reliable machine-learning approaches can drive the success of radiomic applications in clinical care. In this radiomic study, fourteen feature selection methods and twelve classification methods were examined in terms of their performance and stability for predicting overall survival. A total of 440 radiomic features were extracted from pre-treatment computed tomography (CT) images of 464 lung cancer patients. To ensure the unbiased evaluation of different machine-learning methods, publicly available implementations along with reported parameter configurations were used. Furthermore, we used two independent radiomic cohorts for training ( $n=310$ patients) and validation ( $n=154$ patients). We identified that Wilcoxon test based feature selection method WLCX (stability $=0.84 \pm 0.05$, $A \cup C=0.65 \pm 0.02$ ) and a classification method random forest RF (RSD $=3.52 \%$, $A \cup C=0.66 \pm 0.03$ ) had highest prognostic performance with high stability against data perturbation. Our variability analysis indicated that the choice of classification method is the most dominant source of performance variation (34.21\% of total variance). Identification of optimal machine-learning methods for radiomic applications is a crucial step towards stable and clinically relevant radiomic biomarkers, providing a non-invasive way of quantifying and monitoring tumor-phenotypic characteristics in clinical practice. 


\section{INTRODUCTION}

'Precision oncology' refers to the customization of cancer care, where practices and/or therapies are being tailored to individual patients. Such customization process can maximize the success of preventive and therapeutic interventions with minimum side effects. Most of the precision oncology related research has centered on the molecular characterization of tumors using genomics based approaches, which require tissue extraction by tumor biopsies. Although several genomics based approaches have successfully been applied in clinical oncology ${ }^{1}$, there are inherent limitations to biopsy based assays. Tumors are spatially and temporally heterogeneous, and repeated tumor biopsies, which increase the risk for a patient, are often required to capture the molecular heterogeneity of tumors. These ethical and clinical challenges related to biopsybased assays, can be addressed by medical imaging, which is a routine practice for cancer diagnosis and staging in clinical oncology. Unlike biopsies, medical imaging is noninvasive and can provide information regarding the entire tumor phenotype, including the intra-tumor heterogeneity. Furthermore, recent advances in high-resolution image acquisition machines and computational hardware allow the detailed and efficient quantification of tumor phenotypic characteristics. Therefore, medical imaging provides unprecedented opportunities for precision oncology.

"Radiomics", an emerging and promising field, hypothesizes that medical imaging provides crucial information regarding tumor physiology, which could be exploited to enhance cancer diagnostics ${ }^{2}$. It provides a comprehensive quantification of tumor phenotypes by extracting and mining large number of quantitative imaging features ${ }^{3}$. Several studies have investigated various radiomic features in terms of their prognostic or predictive abilities and reliability across different clinical settings ${ }^{4-10}$. Different studies have shown the discriminating capabilities of radiomic features for the stratification of tumor histology ${ }^{6}$, tumor grades or stages ${ }^{11}$, and clinical outcomes ${ }^{8,12,13}$. Moreover, some studies have reported the association between radiomic features and the underlying gene expression patterns ${ }^{8,14,15}$.

"Machine-learning" can be broadly defined as computational methods/models using experience (data) to improve performance or make accurate predictions ${ }^{16}$. These programmable computational methods are capable of "learning" from data and hence can automate and improve the prediction process. Predictive and prognostic models with high accuracy, reliability, and efficiency are vital factors driving the success of radiomics. Therefore, it is essential to compare different machine-learning models for radiomics based clinical biomarkers. Like any high-throughput data-mining field, radiomics also underlies the curse of dimensionality ${ }^{17}$, which should be addressed by appropriate feature selection strategies. Moreover, feature selection also helps in reducing overfitting of models (increasing the generalizability). Thus, in order to reduce the dimensionality of radiomic feature space and enhance the performance of radiomics based predictive models, different feature selection methods ${ }^{18}$ should be thoroughly investigated. However, as radiomics is an emerging research field, most of the published studies have only 
assessed the predictive capabilities of radiomic features without putting much emphasis on the comparison of different feature selection and predictive modeling methods. Only few recent studies have investigated the effect of different feature selection and machine learning classification methods on radiomics based clinical predictions ${ }^{19,20}$, but with limited sample sizes. Furthermore, these studies lacked independent validation of the results, which may restrict the generalizability of their conclusions.

In this study, we investigated a large panel of machine-learning approaches for radiomics based survival prediction. We evaluated 14 feature selection methods and 12 classification methods in terms of their predictive performance and stability against data perturbation. These methods were chosen because of their popularity in literature. Furthermore, publicly available implementations along with reported parameter configurations were used in the analysis, which ensured an unbiased evaluation of these methods. Two independent lung cancer cohorts were used for training and validation, with in total image and clinical outcome data of 464 patients. Feature selection and predictive modeling are considered as the important building blocks for high throughput data driven radiomics. Therefore, our investigation could help in the identification of optimal machine-learning approaches for radiomics based predictive studies, which could enhance the applications of non-invasive and cost-effective radiomics in clinical oncology.

\section{METHODS}

\section{Radiomic Features}

A total of 440 radiomic features were used in the analysis. These radiomic features quantified tumor phenotypic characteristics on CT images and are divided into four feature groups: I) tumor intensity, II) shape, III) texture and IV) wavelet features. Tumor intensity based features estimated the first order statistics of the intensity histogram, whereas shape features described the 3D geometric properties of the tumor. Textural features, derived from the gray level co-occurrence (GLCM) ${ }^{21}$ and run length matrices $(G L R L M)^{22}$, quantified the intra-tumor heterogeneity. These textural features were computed by averaging their values over all thirteen directions. Wavelet features are the transformed domain representations of the intensity and textural features. These features were computed on different wavelet decompositions of the original image using a coiflet wavelet transformation. Matlab R2012b (The Mathworks, Natick, MA) was used for the image analysis. Radiomic features were automatically extracted by our in-house developed radiomics image analysis software, which uses an adapted version of CERR (Computational Environment for Radiotherapy Research) ${ }^{23}$ and Matlab for the preprocessing of medical images. Mathematical definitions of all radiomic features, as well as the extraction methods, were previously described ${ }^{8}$. 


\section{Datasets}

In this study, we employed two NSCLC cohorts from the two different institutes of Netherlands: (1) Lung1: 422 NSCLC patients treated at MAASTRO Clinic in Maastricht. (2) Lung2: 225 NSCLC patients treated at Radboud University Medical Center in Nijmegen. CT-scans, manual delineations and clinical data were available for all included patients. More details on the included datasets are described in Supplementary-A. We dichotomized the censored continuous survival data using a cutoff time of 2 years. The patients who lived beyond the cutoff time were labeled as 1, whereas the deceased ones were labeled as 0 . The objective of the study was to stratify patients into these two labeled survival classes. Two-years is considered as a relevant survival time for NSCLC patients and several other studies have designed their prediction models using a survival cutoff of 2 years ${ }^{24-26}$. We excluded the patients, which were followed for less than 2 years. It resulted in 310 patients in training cohort (Lung1) and 154 patients in validation cohort (Lung2). All the features were normalized using Z-score normalization.

\section{Feature Selection Methods}

Fourteen feature selection methods based on filter approaches were used in the analysis (Fisher score (FSCR), Relief (RELF), T-score (TSCR), Chi-square (CHSQ), Wilcoxon (WLCX), Gini index (GINI), Mutual information maximization (MIM), Mutual information feature selection (MIFS), Minimum redundancy maximum relevance (MRMR), Conditional infomax feature extraction (CIFE), Joint mutual information (JMI), Conditional mutual information maximization (CMIM), Interaction capping (ICAP), Double input symmetric relevance (DISR)). In order to improve the readability of this manuscript, we have defined all the acronyms related to feature selection methods in Table-1. We chose these methods mainly because of their popularity in literature, simplicity and computational efficiency. Furthermore, publicly available implementations were readily available for these methods ${ }^{27,28}$, which increases their reusability. Filter methods are feature-ranking methods, which rank the features using a scoring criterion. All filter based feature selection methods can be divided into two categories: univariate methods and multivariate methods. In case of univariate methods, the scoring criterion only depends on the feature relevancy ignoring the feature redundancy, whereas multivariate methods investigate the multivariate interaction within the features and the scoring criterion is a weighted sum of feature relevancy and redundancy. Feature relevancy is a measure of feature's association with the target/outcome variable, whereas feature redundancy is the amount of redundancy present in a particular feature with respect to the set of already selected features. Further description regarding the theoretical formulation of feature selection problem and each of the used feature selection methods can be obtained from Supplementary-B online. 


\section{Classifiers}

In machine-learning, the classification is considered as a supervised learning task of inferring a function from labeled training data ${ }^{16}$. The training data consists of a set of examples, where each example is represented as a pair of an input vector (features) and a desired output value (target or category label). The classification algorithm (classifier) analyzes the training data and infers a hypothesis (function), which can be used for predicting the labels of unseen observations. Many classifiers belonging to different areas of computer science and statistics have been proposed in machine-learning literature $^{29}$. In our study, we used 12 machine-learning classifiers arising from 12 classifier families (Bagging (BAG), Bayesian (BY), Boosting (BST), Decision trees (DT), Discriminant analysis (DA), Generalized linear models (GLM), Multiple adaptive regression splines (MARS), Nearest neighbors (NN), Neural networks (Nnet), Partial least square and principle component regression (PLSR), Random forests (RF), and Support vector machines (SVM)). The acronyms related to classifiers are defined in Table-1. Except of BY, all the classifiers were implemented using $R$ package $\operatorname{caret}^{30}$, which provides a nice interface to access many machine-learning algorithms in R. Furthermore, it also provides a userfriendly framework for training different machine-learning models. Classifiers were trained using the repeated ( 3 repeat iterations) 10 fold cross validation of training cohort (Lung1) and their predictive performance was evaluated in the validation cohort (Lung2) using area under ROC curve (AUC). We used parameter configurations that were previously defined by Fernandez-Delgado et. $\mathrm{al}^{31}$ in a comprehensive comparative study of 179 classifiers and 121 different datasets. We have listed the classification methods along with their parameters and corresponding $\mathrm{R}$ packages in SupplementaryConline.

\section{Analysis}

\section{Predictive Performance of Feature Selection and Classification Methods}

In order to investigate and compare different feature selection and classification methods, we created a three-dimensional parameter grid for the analysis. For each of the 14 feature selection methods, we incrementally selected features ranging from 5 up to 50, with an increment of 5 features $(n=5,10,15,20, \ldots, 50)$. These subsets of selected features were then evaluated by using each of the 12 machine-learning classifiers and area under ROC curves (AUC).

\section{Stability of Feature Selection and Classification Methods}

In order to assess the stability of feature selection methods, we used a stability measure proposed by $\mathrm{Yu}$ et $\mathrm{al}^{32}$ under the hard data perturbation settings ${ }^{33}$. We quantified the stability of a method as the similarity between the results obtained by the same feature selection method, when applied on the two non-overlapping partitions (of size N/2) of the training cohort (Lung1). To compute similarity between the two resultant feature 
sets, a weighted complete bipartite graph was constructed, where the two node sets corresponded to the two sets of selected features. The edge weights were assigned as the absolute Spearman correlation coefficient between the features at the nodes. We then applied the Hungarian algorithm ${ }^{34}$ to identify the maximum weighted matching between the two node sets, and then similarity was quantified as the final matching cost. For each feature selection method, we computed the stability 100 times using a bootstrap approach and reported the median \pm std values in the results.

The empirical stability of a classifier was quantified using the relative standard deviation (RSD \%) and a bootstrap approach. We first selected 30 representative features using the Wilcoxon based feature selection method WLCX and used them to compute the classifier stability. For each classification method, we trained the model on the subsampled training cohort (size $\mathrm{N} / 2$ ) and validated the performance on the validation cohort using AUC. Subsampling of the training cohort was done 100 times using a bootstrap approach. RSD is the absolute value of the coefficient of variation and is often expressed in percentage. Here, it was defined as

$$
R S D=\frac{\sigma_{A U C}}{\mu_{A U C}} * 100
$$

where $\sigma_{A U C}$ and $\mu_{A U C}$ were the standard deviation and mean of the 100 AUC values respectively. It should be noted that higher stability in the case of classifiers corresponds to lower RSD values.

\section{Stability and Predictive Performance}

In order to identify the highly reliable and accurate methods, we used the median values of AUC and stability as thresholds. We created two rank lists based on AUC \& stability and cited the methods as highly accurate and reliable, which ranked in the top half ( $\geq$ median value) of both the ranked lists. Feature selection methods having stability $\geq$ 0.735 (median stability of all feature selection methods) and AUC $\geq 0.615$ (median AUC of all feature selection methods) are considered as highly reliable and accurate methods. Similarly, classification methods having RSD $\leq 5.97$ (median RSD of all classifiers) and $A \cup C \geq 0.61$ (median AUC of all classifiers) are considered as highly reliable and accurate ones.

\section{Experimental Factors Affecting the Radiomics Based Survival Prediction}

There are three main experimental factors, which can potentially affect the prediction of radiomics based survival prediction: feature selection method, classification method and the number of selected features. Multifactor ANOVA was used to quantify the variability in AUC scores contributed by these factors and their interactions. In order to compare the variability contributed by each factor, the estimated variance components were divided by the total variance.

All the analysis was done using $R$ software ( $R$ Core Team, Vienna, Austria) version 3.1.2 and Matlab R2012b (The Mathworks, Natick, MA) with Windows 7. 


\section{RESULTS}

To investigate the machine-learning approaches for prognostic radiomic biomarkers, a total of 440 radiomic features were extracted from the segmented tumor regions of the pre-treatment CT images of two independent NSCLC cohorts. Feature selection and classification training was done using the training cohort Lung1 ( $n=310$ patients), whereas the validation cohort Lung2 ( $n=154$ patients) was used to assess the predictive performance [see Figure 1].

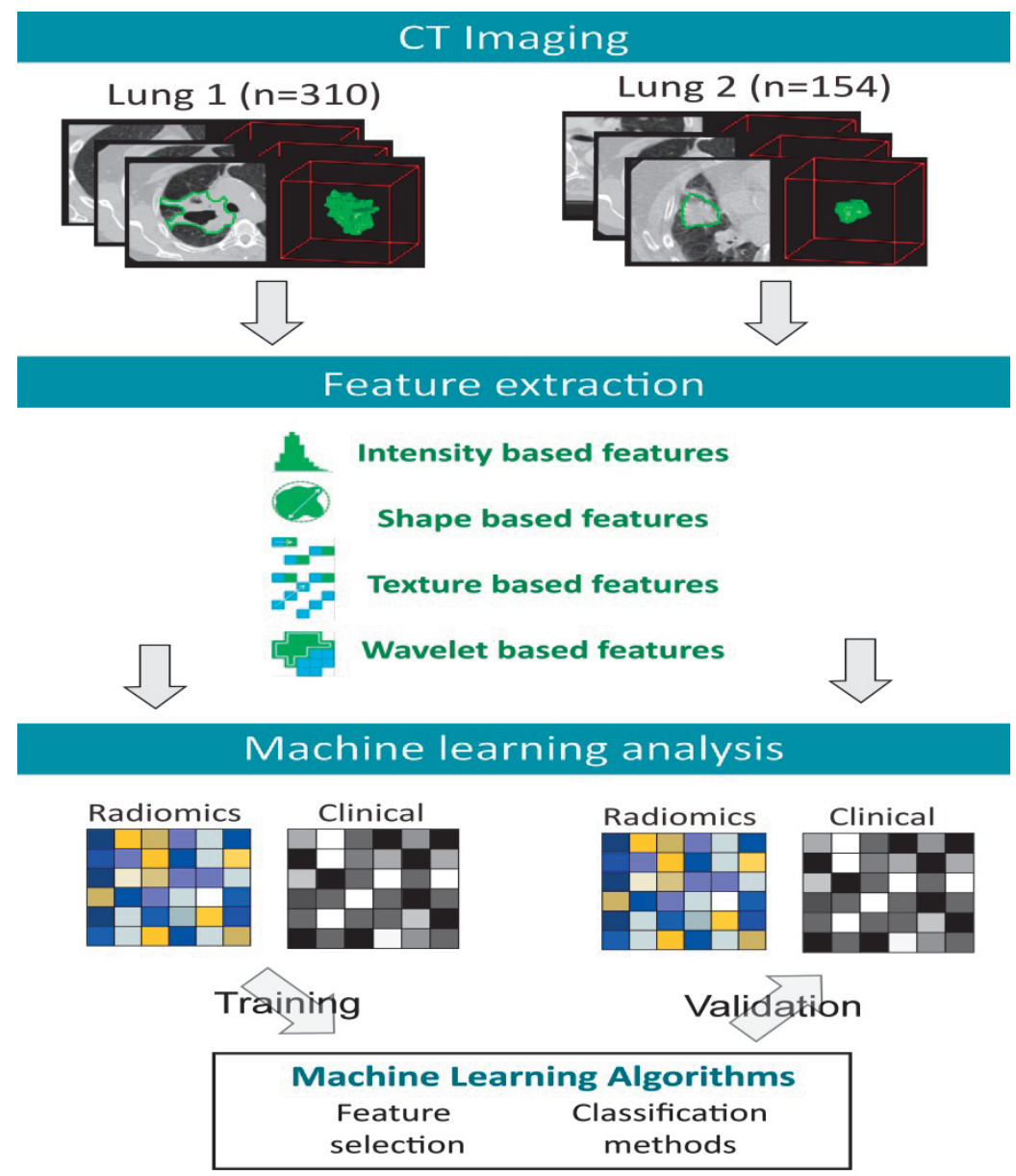

Figure 1. A total of 440 radiomic features were extracted from the segmented tumor regions of the pretreatment CT images of 464 NSCLC patients. Feature selection and classification training was done using the training cohort Lung1 $(n=310)$, whereas Lung2 $(n=154)$ cohort was used as a validation cohort. 


\section{Predictive Performance of the Feature Selection and Classification Methods}

Predictive performance of different feature selection and classification methods was assessed using the area under receiver operator characteristic curve (AUC). Figure 2 depicts the performance of feature selection (in rows) and classification methods (in columns) using 30 selected features, which are the 30 top ranked features, resulted in feature selection. For each classification method, there are 14 AUC values corresponding to the 14 different feature selection methods. We used a median of all 14 AUC values as a representative AUC of a classifier. Similarly, for each feature selection method, a median of 12 AUCs (corresponding to 12 classification methods) is used as a representative AUC. These representative AUC values for the classification and feature selection methods are given in Table 2. For classification methods, random forest (RF) displayed highest predictive performance (AUC: $0.66 \pm 0.03$ ) (median \pm std), whereas decision tree (DT) (AUC: $0.54 \pm 0.04$ ) showed the lowest predictive performance. As far as feature selection methods are concerned, the Wilcoxon test based method WLCX showed highest predictive performance (AUC: $0.65 \pm 0.02$ ), whereas method CHSQ (AUC: $0.60 \pm 0.03$ ) and CIFE (AUC: $0.60 \pm 0.04$ ) had the lowest median AUCs. We repeated the above experiment by varying the number of selected features (range 5-50). Results corresponding to 10,20, 40 and 50 representative (top ranked) features are reported in Supplementary Figures S1, S2, S3 and S4 online. Furthermore, median AUC values over each of the experimental factors (feature selection methods, classification methods and number of selected features) are depicted by the heatmaps in Supplementary Figures S5, S6 and S7 online. Here as well, random forest (RF) (classifier) and Wilcoxon test based method WLCX (feature selection) showed highest median AUCs in majority of cases.

\section{Stability of the Feature Selection and Classification Methods}

We assessed the feature selection methods in terms of their stability against data resampling using the hard data perturbation settings ${ }^{33}$. We observed that MIM was the most stable method (stability $=0.94 \pm 0.02$ ) (median \pm std) followed by RELIEF (stability $=0.91 \pm 0.05$ ) and WLCX (stability = $0.84 \pm 0.05$ ), whereas GINI (stability $=0.68 \pm 0.10$ ), $\mathrm{JMI}$ (stability $=0.68 \pm 0.05), \mathrm{CHSQ}$ (stability $=0.69 \pm 0.09$ ), DISR (stability $=0.69 \pm 0.05$ ) and CIFE (stability $=0.69 \pm 0.05$ ) showed comparatively low stability [Table 2].

Empirical stability of classification methods was quantified using the relative standard deviation (RSD) and a bootstrap approach. We observed that BY was the most stable classification method (RSD $=0.86 \%$ ) followed by GLM (RSD = 2.19\%), PLSR (RSD = $2.24 \%)$ and RF (RSD $=3.52 \%)$. BST had the highest relative standard deviation in AUC scores (RSD $=8.23 \%$ ) and hence the lowest stability among the classification methods. RSD (\%) values corresponding to all 12 classifiers are reported in Table 2. 

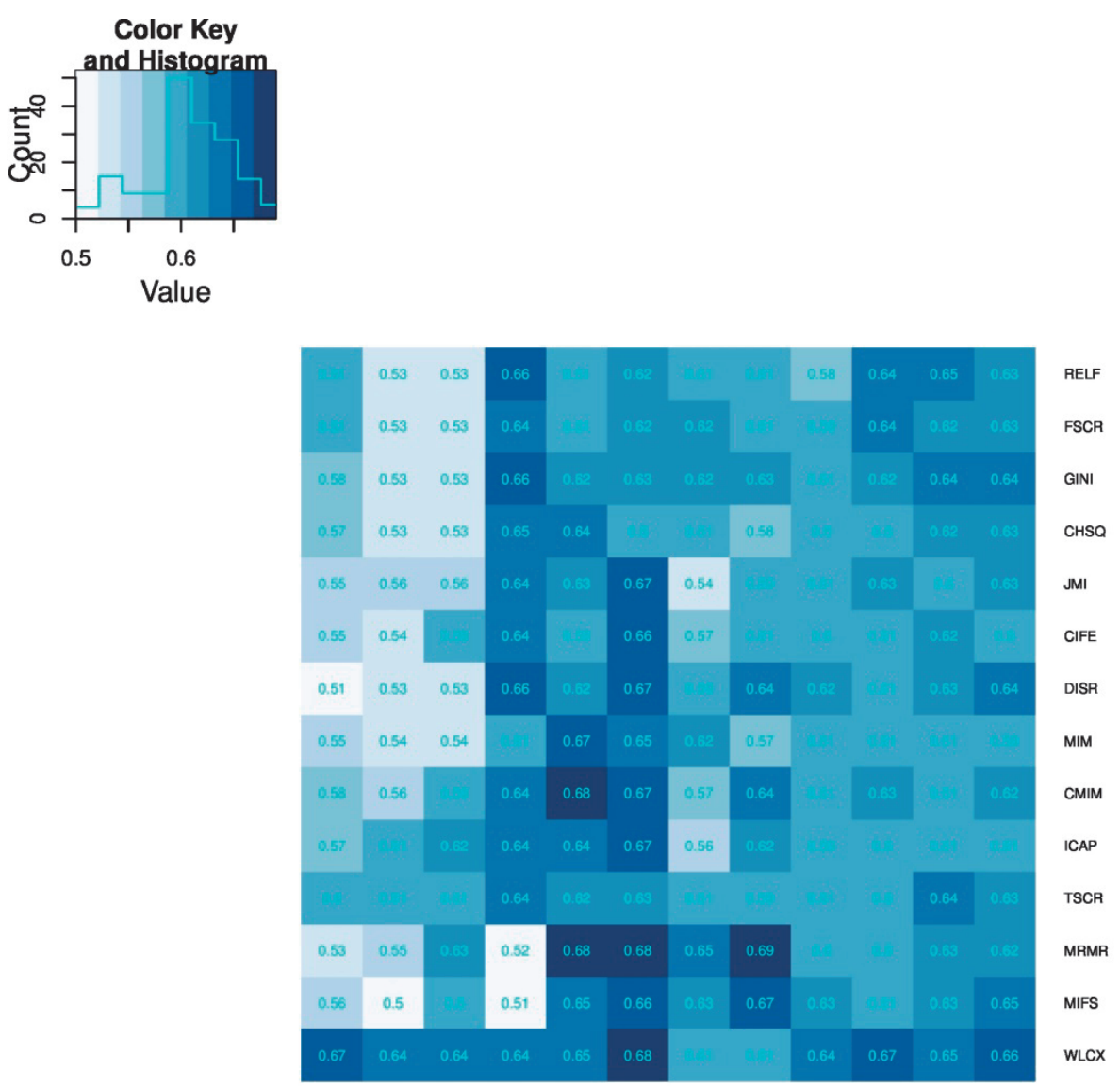

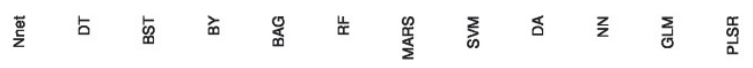

Figure 2. Heatmap depicting the predictive performance (AUC) of feature selection (in rows) and classification (in columns) methods. It can be observed that RF, BAG and BY classification methods and feature selection methods WLCX, MRMR and MIFS shows relatively high predictive performance in many cases.

\section{Stability and Predictive Performance}

Scatterplots in Figure 3 assesses the stability and prediction performance. It can be observed that feature selection methods WLCX (stability $=0.84 \pm 0.05, A \cup C=0.65 \pm$ 0.02 ), MIFS (stability $=0.8 \pm 0.03, A \cup C=0.63 \pm 0.03$ ), MRMR (stability $=0.74 \pm 0.03$, AUC $=0.63 \pm 0.03$ ) and FSCR (stability $=0.78 \pm 0.08$, AUC $=0.62 \pm 0.04$ ) should be preferred as their stability and predictive performance was higher than the corresponding median values across all feature selection methods (stability $=0.735, A \cup C=0.615$ ). Similarly for classification methods, RF (RSD $=3.52 \%, A \cup C=0.66 \pm 0.03), B Y(R S D=$ $0.86 \%, A \cup C=0.64 \pm 0.05), B A G(R S D=5.56 \%, A \cup C=0.64 \pm 0.03), G L M(R S D=2.19 \%$, $A \cup C=0.63 \pm 0.02)$, and PLSR (RSD $=2.24 \%, A \cup C=0.63 \pm 0.02)$, the stability and predic- 
tive performance was higher than the corresponding median values (RSD $=5.93 \%$, AUC $=0.61$. .
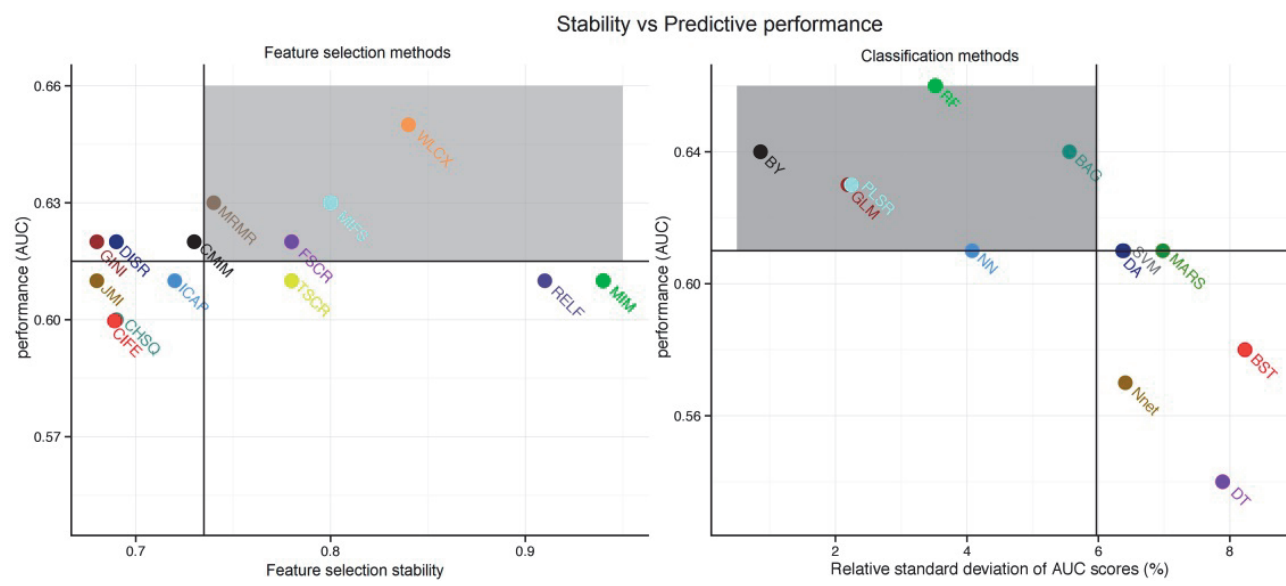

Figure 3. Scatterplots between the stability and predictive performance (AUC) of feature selection (FS) (Left) and classification methods (CF) (right). Feature selection methods having stability $\geq 0.735$ (median stability of FS) and AUC $\geq 0.615$ (median AUC of FS) are considered as highly reliable and predictive methods. Similarly, classification methods having RSD $\leq 5.97$ (median RSD of CF) and AUC $\geq 0.61$ (median AUC of CF) are considered as highly reliable and accurate ones. Highly reliable and predictive methods are displayed in a gray square region.

\section{Experimental Factors Affecting the Radiomics Based Survival Prediction}

To quantify the effects of the three experimental factors (feature selection methods, classification methods and the number of selected features), we performed multifactor analysis of variance (ANOVA) on AUC scores. We observed that all three experimental parameters and their interactions are the significant factors affecting the prediction performance [Figure 4]. Classification method was the most dominant source of variability as it explained $34.21 \%$ of the total variance in AUC scores. Feature selection accounted for the $6.25 \%$, whereas interaction of classifier \& feature selection explained $23.03 \%$ of the total variation. Size of the selected (representative) feature subset only shared $1.65 \%$ of the total variance [Figure 4 ]. 


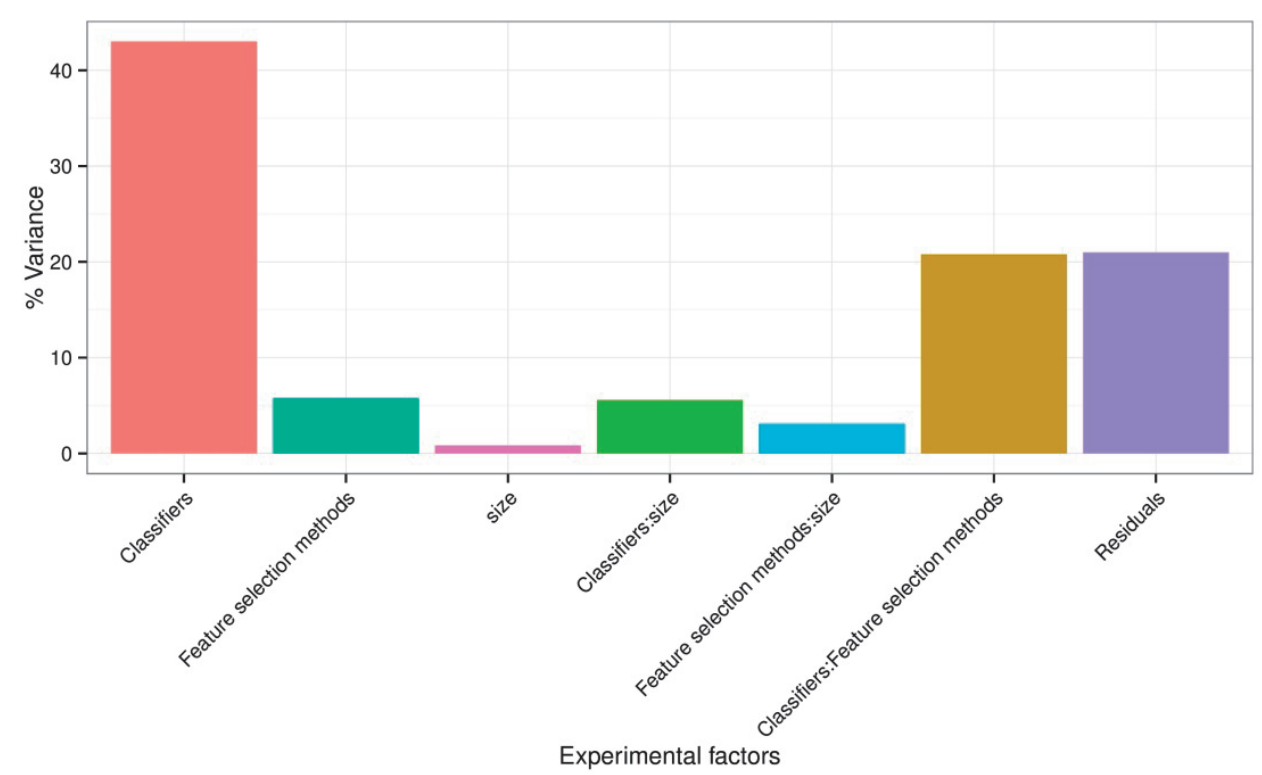

Figure 4. Variation of AUC explained by the experimental factors and their interactions. It can be observed that classification method was the most dominant source of variability. Size of the selected (representative) feature subset shared the least of the total variance.

\section{DISCUSSION}

Medical imaging is a routinely used and easily accessible source of information in clinical oncology. It serves as a non-invasive and cost-effective cancer diagnostic tool. Radiomics employs the medical imaging data for the customization of cancer care and hence adds a new and promising dimension to precision oncology $y^{2,3,8}$. Moreover, it can also capture the intra-tumor heterogeneity, which is often considered as an important biomarker in oncology $y^{12,35-37}$. A number of studies have built radiomics based predictive models for various clinical factors (tumor grades, survival outcomes, treatment response, etc. $)^{12}$. For the successful realization of radiomics based predictive analyses, it is required to evaluate and compare different feature selection and predictive modeling methods, which was the primary objective of this study.

Various feature selection methods have been employed for high-throughput data mining problems ${ }^{38}$. In general, feature selection methods are categorized into three main categories: (1) filter methods (2) wrapper methods and (3) embedded methods. In this study, we investigated 14 different filter based approaches for radiomics based survival prediction. We only used filter-based approaches because they are computationally more efficient and less prone to overfitting than the wrapper and embedded methods $^{18,27}$. Furthermore, unlike wrapper and embedded methods, filter methods are classifier independent. Thus, they allow separation of the modeling and feature selec- 
tion component of the predictive analysis, which increases the generalizability of each component and hence the overall analysis.

We also investigated 12 machine-learning classification methods belonging to 12 different classifier families. Many classifiers have been proposed in the machine-learning literature. Theoretically speaking, these classifiers belong to different fields (classifier families) of computer science and statistics. Therefore, it could really be difficult to understand the underlying assumptions of each and every classifier and tune the parameters in an unbiased manner. The parameter tuning could be biased by user's more (or lack of) expertise with some classifiers over the others. Usually, the studies, which propose a new classifier, only compare it to the reference classifiers of same family excluding the other classifier families. Even if classifiers belonging to different families are considered for comparison, these reference classifiers are usually implemented using simple tools and with limited parameter configurations while carefully tuning the proposed classifier. These could consequently bias the results in favor of the proposed classifiers $^{31}$. In our study, we are not proposing any new classifier and we have used the same implementation tool (R package caret) for all the classifiers. Furthermore, to ensure unbiased usage of classifiers, we used parameter configurations that were previously defined by Fernandez-Delgado et.al ${ }^{31}$, in an exhaustive study of comparing 179 classifiers over 121 different datasets. These parameter configurations were selected from the literature and have been previously validated on a large number (121) of datasets belonging to different fields. Furthermore, in our study, the parameters were tuned using the repeated cross validation of training data only. Hence, our experimental design allowed us to evaluate different classification methods in an unbiased manner.

Our results show that the Wilcoxon test based feature selection method WLCX yields the highest predictive performance with the majority of classifiers. Interestingly, WLCX is a simple univariate method based on ranks, which does not take into account the redundancy of selected features during feature ranking. The majority of feature selection methods gave highest predictive performance when used with the random forest (RF) classifier. One could argue that with different parameter configurations, the performance of classification methods may improve further. An exhaustive parameter tuning could be investigated for evaluating the improvement of prediction performance. However, the required computational resources and high time complexity can hinder the exhaustive search. We expect that future radiomic studies focusing on different clinical outcomes and similar analysis framework could provide better understanding in this regard. A limited number of methods, which are consistently high performing across different radiomic studies, could be further assessed with an exhaustive parameter tuning. Nevertheless, It should be noted that random forests (RF) have displayed high predictive performance in several other biomedical and other domain applications as well ${ }^{31}$. These results indicate that choosing the WLCX feature selection method and/or RF classification method increases predictive performance in radiomics.

Results related to our stability analysis provide another dimension for choosing the feature selection and classification methods. Depending upon the applications, one may 
give importance to the predictive performance or stability and accordingly opt for the required method. Results related to multifactor ANOVA indicated that the classification method is the most dominant source of variation in the prediction performance (AUC) and hence should be chosen carefully. Size of the selected feature subset contributed the least in the total variation of AUC.

Only few studies have investigated and compared different feature selection and machine-learning modeling methods for radiomics based clinical predictions ${ }^{19,20}$. Recently, Hawkins et.al ${ }^{19}$ have compared four different feature selection and classification methods for CT based survival prediction of NSCLC patients. This study, however, was limited by the small cohort size as the final results were obtained on only 40 patients. Furthermore, it also lacked an independent validation of the results. On the contrary, two independent radiomic cohorts of sizes 310 and 154 patients were used in our analysis and an independent validation of the results was reported.

Our radiomic analysis is focused on the prediction of two-year patient survival in NSCLC patients. It provides an unbiased evaluation of different machine-learning methods of feature selection and classification. It could be considered as a reference for the future radiomics based predictive studies. Our results indicated that choosing Wilcoxon test based feature selection method WLCX and/or random forest (RF) classification method gives highest performance for radiomics based survival prediction. Furthermore, these methods also turned out reasonably stable against data perturbation and hence they could be preferred for radiomics based predictive studies. These results should be further tested in other radiomics based predictive studies, with different imaging modalities and in different cancer types.

It has been previously shown that for NSCLC patients, statistical models based on patient's tumor and treatment characteristics provide significantly better predictions than the human expert ${ }^{24}$. Moreover, several other studies have highlighted the limitation of doctors' prognostic capability for terminally ill cancer patients ${ }^{39-41}$. The predictions of human experts can suffer from inter-observer variability. On the contrary, statistical models could make the prediction system more deterministic if the parameter configurations and the training framework are fixed.

The potential clinical utility of radiomics based prognostic models has been stated in previous study ${ }^{8}$. With expanding radiomics cohorts and feature dimensions, we expect higher prediction performance in future radiomic studies. Furthermore, the integrative studies like radiomics-genomics in combination with standard clinical covariates could also improvise the prediction performance and further validate the utility of these methods in clinical practice. Overall, our analysis is a step forward towards the enhancements of radiomics based clinical predictions. 


\section{TABLES}

Table-1. Table defining the acronyms related to the used feature selection and classification methods.

\begin{tabular}{|c|c|c|c|}
\hline $\begin{array}{l}\text { Classification } \\
\text { method acronym }\end{array}$ & Classification method name & $\begin{array}{l}\text { Feature Selection } \\
\text { method acronym }\end{array}$ & $\begin{array}{l}\text { Feature selection method } \\
\text { name }\end{array}$ \\
\hline Nnet & Neural network & RELF & Relief \\
\hline DT & Decision Tree & FSCR & Fisher score \\
\hline BST & Boosting & GINI & Gini index \\
\hline BY & Bayesian & $\mathrm{CHSQ}$ & Chi-square score \\
\hline BAG & Bagging & $\mathrm{JMI}$ & Joint mutual information \\
\hline RF & Random Forset & CIFE & $\begin{array}{l}\text { Conditional infomax } \\
\text { feature extraction }\end{array}$ \\
\hline MARS & Multi adaptive regression splines & DISR & $\begin{array}{l}\text { Double input symmetric } \\
\text { relevance }\end{array}$ \\
\hline SVM & Support vector machines & MIM & $\begin{array}{l}\text { Mutual information } \\
\text { maximization }\end{array}$ \\
\hline DA & Discriminant analysis & CMIM & $\begin{array}{l}\text { Conditional mutual } \\
\text { information maximization }\end{array}$ \\
\hline NN & Neirest neighbour & ICAP & Interaction capping \\
\hline GLM & Generalized linear models & TSCR & T-test score \\
\hline PLSR & $\begin{array}{l}\text { Partial least squares and prinicipal } \\
\text { componenet regression }\end{array}$ & MRMR & $\begin{array}{l}\text { Minimum redundancy } \\
\text { maximum relevance }\end{array}$ \\
\hline- & - & MIFS & $\begin{array}{l}\text { Mutual information } \\
\text { feature selection }\end{array}$ \\
\hline- & - & WLCX & Wilcoxon \\
\hline
\end{tabular}

Table 2. Table describing the median values of AUC and stability for different Classification and Feature Selection methods.

\begin{tabular}{llllll}
\hline $\begin{array}{l}\text { Classification } \\
\text { method }\end{array}$ & AUC & RSD $\%$ & $\begin{array}{l}\text { Feature Selection } \\
\text { method }\end{array}$ & AUC & Stability \\
\hline Nnet & $0.57 \pm 0.04$ & 6.41 & RELF & $0.61 \pm 0.04$ & $0.91 \pm 0.05$ \\
DT & $0.54 \pm 0.04$ & 7.89 & FSCR & $0.62 \pm 0.04$ & $0.78 \pm 0.08$ \\
BST & $0.58 \pm 0.04$ & 8.23 & GINI & $0.62 \pm 0.04$ & $0.68 \pm 0.10$ \\
BY & $0.64 \pm 0.05$ & 0.86 & CHSQ & $0.60 \pm 0.04$ & $0.69 \pm 0.09$ \\
BAG & $0.64 \pm 0.03$ & 5.56 & JMI & $0.61 \pm 0.04$ & $0.68 \pm 0.05$ \\
RF & $0.66 \pm 0.03$ & 3.52 & CIFE & $0.60 \pm 0.03$ & $0.69 \pm 0.05$ \\
MARS & $0.61 \pm 0.03$ & 6.98 & DISR & $0.62 \pm 0.05$ & $0.69 \pm 0.05$ \\
SVM & $0.61 \pm 0.03$ & 6.39 & MIM & $0.61 \pm 0.04$ & $0.94 \pm 0.02$ \\
DA & $0.61 \pm 0.02$ & 6.37 & CMIM & $0.62 \pm 0.04$ & $0.73 \pm 0.04$ \\
NN & $0.61 \pm 0.02$ & 4.08 & ICAP & $0.61 \pm 0.03$ & $0.72 \pm 0.04$ \\
GLM & $0.63 \pm 0.02$ & 2.19 & TSCR & $0.61 \pm 0.02$ & $0.78 \pm 0.12$ \\
PLSR & $0.63 \pm 0.02$ & 2.24 & MRMR & $0.63 \pm 0.06$ & $0.74 \pm 0.03$ \\
- & - & - & MIFS & $0.63 \pm 0.06$ & $0.8 \pm 0.03$ \\
- & - & WLCX & $0.65 \pm 0.02$ & $0.84 \pm 0.05$ \\
\hline
\end{tabular}




\section{ACKNOWLEDGEMENT}

Authors acknowledge financial support from the National Institute of Health (NIH-USA U24CA194354, and NIH-USA U01CA190234), EU 7th framework program (EURECA, ARTFORCE), Kankeronderzoekfonds Limburg from the Health Foundation Limburg and the Dutch Cancer Society (KWF UM 2009-4454, KWF MAC 2013-6425).

\section{SUPPLEMENTARY INFORMATION}

http://www.nature.com/article-assets/npg/srep/2015/150817/srep13087/extref/ srep13087-s1.pdf 


\section{REFERENCES}

1 Doroshow, J. \& Kummar, S. Translational research in oncology-10 years of progress and future prospects. Nat. Rev. Clin. Oncol. 11, 649 (2014).

2 Lambin, P. et al. Predicting outcomes in radiation oncology-multifactorial decision support systems. Nat. Rev. Clin. Oncol. 10, 27-40 (2013).

3 Lambin, P. et al. Radiomics: extracting more information from medical images using advanced feature analysis. Eur. J. of Cancer 48, 441-446 (2012).

4 Coroller, T. P. et al. CT-based radiomic signature predicts distant metastasis in lung adenocarcinoma. Radiothe. Oncol. (2015), doi: http://dx.doi.org/10.1016/j.radonc.2015.02.015 (2015).

5 Cook, G. J. et al. Are Pretreatment 18F-FDG PET Tumor Textural Features in Non-Small Cell Lung Cancer Associated with Response and Survival After Chemoradiotherapy? J. Nucl. Med. 54, 19-26 (2013).

6 Ganeshan, B. et al. Non-small cell lung cancer: histopathologic correlates for texture parameters at CT. Radiology 266, 326-336 (2013).

7 Gevaert, O. et al. Glioblastoma multiforme: exploratory radiogenomic analysis by using quantitative image features. Radiology 273, 168-174 (2014).

8 Aerts, H. J. et al. Decoding tumour phenotype by noninvasive imaging using a quantitative radiomics approach. Nat. Commun. 5 (2014).

9 Leijenaar, R. T. et al. Stability of FDG-PET Radiomics features: An integrated analysis of test-retest and inter-observer variability. Acta Oncol. 52, 1391-1397 (2013).

10 Parmar, C. et al. Robust radiomics feature quantification using semiautomatic volumetric segmentation. PLOS ONE 9, e102107 (2014).

11 Ganeshan, B., Abaleke, S., Young, R. C., Chatwin, C. R. \& Miles, K. A. Texture analysis of non-small cell lung cancer on unenhanced computed tomography: initial evidence for a relationship with tumour glucose metabolism and stage. Cancer Imaging 10, 137 (2010).

12 Alic, L., Niessen, W. J. \& Veenland, J. F. Quantification of heterogeneity as a biomarker in tumor imaging: a systematic review. PLOS ONE 9, e110300 (2014).

13 Jain, R. et al. Outcome prediction in patients with glioblastoma by using imaging, clinical, and genomic biomarkers: focus on the nonenhancing component of the tumor. Radiology 272, 484-493 (2014).

14 Nicolasjilwan, M. et al. Addition of MR imaging features and genetic biomarkers strengthens glioblastoma survival prediction in TCGA patients. J. Neuroradiol. (2014), doi:10.1016/j.neurad.2014.02.006. (2014).

15 Segal, E. et al. Decoding global gene expression programs in liver cancer by noninvasive imaging. Nat. biotechnol. 25, 675-680 (2007).

16 Mohri, M., Rostamizadeh, A. \& Talwalkar, A. Foundations of machine learning. Ch. 1, 1-3, (MIT press, 2012).

17 Pękalska, E. \& Duin, R. P. The dissimilarity representation for pattern recognition: foundations and applications. Vol. 64 (World Scientific, 2005).

18 Guyon, I. \& Elisseeff, A. An introduction to variable and feature selection. J. Mach. Learn. Res. 3, 11571182 (2003).

19 Hawkins, S. H. et al. Predicting Outcomes of Nonsmall Cell Lung Cancer Using CT Image Features. IEEE Access 2, 1418 - 1426 (2014).

20 Basu, S. et al. in Systems, Man, and Cybernetics (SMC), 2011 IEEE International Conference on. 13061312 (IEEE).

21 Haralick, R. M., Shanmugam, K. \& Dinstein, I. H. Textural features for image classification. IEEE Trans. Syst., Man Cybern. 6, 610-621 (1973).

22 Galloway, M. M. Texture analysis using gray level run lengths. Comput. Vision Graph. 4, 172-179 (1975).

23 Deasy, J. O., Blanco, A. I. \& Clark, V. H. CERR: a computational environment for radiotherapy research. Med. Phys. 30, 979-985 (2003).

24 Oberije, C. et al. A prospective study comparing the predictions of doctors versus models for treatment outcome of lung cancer patients: a step toward individualized care and shared decision making. Radiothe. Oncol. 112, 37-43 (2014). 
25 Hoang, T., Xu, R., Schiller, J. H., Bonomi, P. \& Johnson, D. H. Clinical model to predict survival in chemonaive patients with advanced non-small-cell lung cancer treated with third-generation chemotherapy regimens based on Eastern Cooperative Oncology Group data. J. Clin. Oncol. 23, 175-183 (2005).

26 Cistaro, A. et al. Prediction of 2 years-survival in patients with stage I and II non-small cell lung cancer utilizing 18F-FDG PET/CT SUV quantifica. Radiol. oncol. 47, 219-223 (2013).

27 Brown, G., Pocock, A., Zhao, M.-J. \& Luján, M. Conditional likelihood maximisation: a unifying framework for information theoretic feature selection. J. Mach. Learn. Res. 13, 27-66 (2012).

28 Zhao, Z. et al. Advancing feature selection research. ASU feature selection repository (2010).

29 Kotsiantis, Sotiris B., Ioannis D. Zaharakis, and Panayiotis E. Pintelas. Machine learning: a review of classification and combining techniques. Artif. Intell. Rev. 26.3 (2006): 159-190.

30 Kuhn, M. Building predictive models in R using the caret package. J. Stat. Softw. 28, 1-26 (2008).

31 Fernández-Delgado, M., Cernadas, E., Barro, S. \& Amorim, D. Do we need hundreds of classifiers to solve real world classification problems? J. Mach. Learn. Res. 15, 3133-3181 (2014).

$32 \mathrm{Yu}, \mathrm{L}$., Ding, C. \& Loscalzo, S. in Proceedings of the 14th ACM SIGKDD international conference on Knowledge discovery and data mining. 803-811 (ACM).

33 Haury, A.-C., Gestraud, P. \& Vert, J.-P. The influence of feature selection methods on accuracy, stability and interpretability of molecular signatures. PLOS ONE 6, e28210 (2011).

34 Kuhn, H. W. The Hungarian method for the assignment problem. Naval Res. Logis. Q. 2, 83-97 (1955).

35 Fisher, R., Pusztai, L. \& Swanton, C. Cancer heterogeneity: implications for targeted therapeutics. Br. J Cancer 108, 479-485 (2013).

$36 \mathrm{Ng}, \mathrm{C}$., Pemberton, H. \& Reis-Filho, J. Breast cancer intratumor genetic heterogeneity: causes and implications. Expert Rev. Anticancer Ther. 12, 1021-1032 (2012).

37 Brown, J. R., DiGiovanna, M. P., Killelea, B., Lannin, D. R. \& Rimm, D. L. Quantitative assessment Ki-67 score for prediction of response to neoadjuvant chemotherapy in breast cancer. Lab. Invest. 94, 98-106 (2014).

38 Bolón-Canedo, V., Sánchez-Maroño, N., Alonso-Betanzos, A., Benítez, J. \& Herrera, F. A review of microarray datasets and applied feature selection methods. Inform. Sciences 282, 111-135 (2014).

39 Christakis, N. A., Smith, J. L., Parkes, C. M. \& Lamont, E. B. Extent and determinants of error in doctors' prognoses in terminally ill patients: prospective cohort studyCommentary: Why do doctors overestimate? Commentary: Prognoses should be based on proved indices not intuition. Bmj 320, 469-473 (2000).

40 Glare, P. et al. A systematic review of physicians' survival predictions in terminally ill cancer patients. Bmj 327, 195 (2003).

41 Clément-Duchêne, C., Carnin, C., Guillemin, F. \& Martinet, Y. How accurate are physicians in the prediction of patient survival in advanced lung cancer? Oncologist 15, 782-789 (2010). 


\section{Chapter}

\section{Radiomic machine learning classifiers for prognostic biomarkers of Head \& Neck cancer}

Published in: Frontiers in Oncology 5, 272; 2015.

Radiomic machine learning classifiers for prognostic biomarkers of Head \& Neck cancer Chintan Parmar*, Patrick Grossmann*, Derek Rietveld, Michelle M. Rietbergen, Philippe Lambin, Hugo J.W.L. Aerts

*These authors contributed equally to this work 


\section{ABSTRACT}

Introduction: "Radiomics" extracts and mines large number of medical imaging features in a non-invasive and cost-effective way. The underlying assumption of radiomics is that these imaging features quantify phenotypic characteristics of entire tumor. In order to enhance applicability of radiomics in clinical oncology, highly accurate and reliable machine learning approaches are required. In this radiomic study, thirteen feature selection methods and eleven machine learning classification methods were evaluated in terms of their performance and stability for predicting overall survival in head and neck cancer patients.

Methods: Two independent head and neck cancer cohorts were investigated. Training cohort HN1 consisted 101 HNSCC patients. Cohort HN2 ( $n=95$ ) was used for validation. A total of 440 radiomic features were extracted from the segmented tumor regions in $\mathrm{CT}$ images. Feature selection and classification methods were compared using an unbiased evaluation framework.

Results: We observed that the three feature selection methods MRMR ( $A \cup C=0.69$, Stability $=0.66)$, MIFS (AUC $=0.66$, Stability $=0.69)$, and CIFE (AUC $=0.68$, Stability $=$ 0.7 ) had high prognostic performance and stability. The three classifiers BY (AUC $=0.67$, RSD $=11.28), \operatorname{RF}(A \cup C=0.61, R S D=7.36)$, and $N N(A \cup C=0.62, R S D=10.52)$ also showed high prognostic performance and stability. Analysis investigating performance variability indicated that the choice of classification method is the major factor driving the performance variation (29.02\% of total variance).

Conclusions: Our study identified prognostic and reliable machine learning methods for the prediction of overall survival of head and neck cancer patients. Identification of optimal machine-learning methods for radiomics based prognostic analyses could broaden the scope of radiomics in precision oncology and cancer care. 


\section{INTRODUCTION}

The emergence of "Radiomics" (1) has expanded the scope of medical imaging in clinical oncology. Radiomics focuses on extracting and mining a large number of medical imaging features. It is hypothesized that these imaging features are enriched with crucial information regarding tumor phenotype $(1,2)$. These features provide a comprehensive characterization of entire tumors and hence are likely to capture the intra-tumor heterogeneity. It has been stated that intra-tumor heterogeneity could have profound implications in clinical predictions (e.g., treatment response, survival outcomes, disease progression, etc.) and therefore it is considered as a crucial factor for precision oncology and related research (3-6). Several studies have assessed various radiomic features in different cancer types and with respect to different imaging modalities $(2,7-11)$. Some studies have investigated the reproducibility/variability of radiomic features across different clinical settings (2, 12-14). Moreover, several other studies have reported significant predictive/prognostic power of radiomic features. It has been shown that radiomic features are associated with tumor histology (15-17), tumor grades or stages (16), patient survival (2, 7, 18-20), metabolism (21), and various other clinical outcomes (7, 16, 22, 23). Furthermore, some radio-genomic studies have reported associations between radiomic features and underlying gene expression patterns $(2,9,11,24,25)$. These reports indicate that radiomics could improve individualized treatment selection and monitoring. Furthermore, unlike most of the genomic-based approaches, radiomics is non-invasive and relatively cost effective $(2,26)$. Therefore, radiomics is a novel and promising step forward towards the realization of precision oncology.

Predictive and prognostic models are important part of radiomics (27). Highly accurate and reliable models are desired to improve decision support in clinical oncology. Machine learning could help in this regard. Machine learning can be broadly defined as computational methods/models using data to improve performance or make accurate predictions (28). These programmable methods can "learn" from the data and hence automate and improve the prediction process. Therefore, it is essential to compare different machine learning models for precision oncology and hence also for radiomics based clinical biomarkers. Recent advances in medical image acquisition technologies allow higher resolution tumor imaging and facilitate detailed quantification of tumor phenotype. The feature dimensions of radiomics are increasing rapidly. One of the issues with high dimensional feature space is the "curse of dimensionality" (29). Large number of features with limited sample size could hinder the predictive/prognostic power of a model. Feature/variable selection is one of the ways to tackle the curse of dimensionality. Therefore, different feature selection methods (29) should be thoroughly investigated for radiomics based prognostic analyses. However, as radiomics is an emerging field of research, not sufficient effort could be made towards assessing the impact of different machine learning methods. The majority of the radiomics based studies have only assessed the discriminating power of radiomic features without evaluating alternative prediction/prognostic models. 
Only few recent studies have compared different feature selection and classification methods on radiomics based clinical predictions $(15,20)$, but with limited sample sizes and also without independent validation. In a recently published radiomic study (30), a large panel of feature selection and machine learning classification methods was evaluated in two independent cohorts of patients with non-small cell lung cancer (NSCLC). They proposed an unbiased framework for comparing different feature selection and classification methods using publicly available implementations $(31,32)$ and reported parameter configurations (33).

In this study, we assessed a large panel of machine learning methods for overall survival prediction of head and neck cancer (HNSCC) patients. Two independent HNSCC cohorts totaling 196 patients were used in the analysis. Feature selection and classification training was done using training cohort $\mathrm{HN} 1$ and the prediction performance was evaluated in the validation cohort HN2. All the feature selection and classification methods were evaluated in terms of their prognostic ability and stability against data perturbation. Machine learning methods having high prognostic/predictive power and stability are desired for radiomics based analyses. Such methods could enhance the applications of non-invasive and cost-effective radiomics in cancer care.

\section{MATERIALS AND METHODS}

\section{Radiomic Features}

A total of 440 radiomic features that quantified tumor phenotypic characteristics on CT were used in this analysis. These radiomic features are divided into four feature groups: I) tumor intensity, II) shape, III) texture and IV) wavelet features. Intensity features are computed using the first-order statistics of voxel intensity histogram. 3D geometric properties of tumors are captured using the shape based features. Textural features are quantified using the gray level co-occurrence (GLCM) (34) and run length matrices (GLRLM) (35). These textural features were computed by averaging their values over all thirteen directions. Intensity statistics and textural features were also recomputed after decomposing the original image into different wavelet decompositions. Image analysis and automatic feature extraction was carried out using our in-house developed radiomics image analysis software, which uses an adapted version of CERR (Computational Environment for Radiotherapy Research) (36) and Matlab R2012b (The Mathworks, Natick, MA) for the preprocessing of medical images. Mathematical definitions of all radiomic features, as well as the extraction methods, were previously described (2).

\section{Datasets}

In this study, we analyzed two HNSCC cohorts from the two different institutes of Netherlands: (1) HN1: 136 HNSCC patients treated at MAASTRO Clinic, Maastricht. (2) HN2: 
95 HNSCC patients treated at VU University Medical Center (VUMC), Amsterdam. CTscans, manual delineations and clinical data were available for all included patients. This analysis was carried out in accordance with Dutch law. The Institutional Review Boards of both the participating centers approved the studies: H\&N1 MAASTRO Clinic Maastricht, The Netherlands) and H\&N2 (VU University Medical Center (VUMC), Amsterdam, The Netherlands). More details on the included datasets can be obtained from a previous study (2). We dichotomized the censored continuous survival data using a cutoff time of 3 years. Patients who survived beyond the cutoff time were labeled as 1 , whereas the deceased ones were labeled as 0 . The objective of the study was to stratify patients into these two labeled survival classes using different machine learning classifiers. We used 3 years survival cut-off because it resulted in reasonable event ratios (37\% for HN1, 34\% for HN2) in the cohorts. We excluded patients that were followed up for less than 3 years. This resulted in 101 patients in the training cohort (HN1) and 95 patients in the validation cohort (HN2). All features were standardized using Z-score standardization.

\section{Feature Selection Methods}

As described in a previously published study (30), fourteen feature selection methods based on filter approaches were used in the analysis: Fisher score (FSCR), Relief (RELF), T-score (TSCR), Chi-square (CHSQ), Wilcoxon (WLCX), Gini index (GINI), Mutual information maximization (MIM), Mutual information feature selection (MIFS), Minimum redundancy maximum relevance (MRMR), Conditional infomax feature extraction (CIFE), Joint mutual information (JMI), Conditional mutual information maximization (CMIM), Interaction capping (ICAP), and Double input symmetric relevance (DISR). However, the method $\mathrm{CHSQ}$ did not run according to our experimental design. CHSQ was not able to select the required number of features due to the smaller size of training cohort. We thus removed it from further analysis. The acronyms related to the feature selection methods are defined in Table-1. Publicly available implementations were used for these methods $(31,32)$. Detailed description regarding these methods can be obtained from (30).

\section{Classifiers}

As described earlier (30), we investigated twelve machine learning classifiers belonging to the twelve classifier families Bagging (BAG), Bayesian (BY), Boosting (BST), Decision trees (DT), Discriminant analysis (DA), Generalized linear models (GLM), Multiple adaptive regression splines (MARS), Nearest neighbors (NN), Neural networks (Nnet), Partial least square and principle component regression (PLSR), Random forests (RF), and Support vector machines (SVM). In our experimental settings, classifier DA generated computation error in the majority of cases. This could be due to the smaller training cohort. Therefore, we removed DA from further analysis and used the remaining eleven classi- 
fiers. The acronyms related to the classifiers are defined in Table-1. All classifiers were implemented using the R package caret (version 6.0-47) (37), which provides a nice interface to access many machine-learning algorithms in R. Classifiers were trained using the repeated ( 3 repeat iterations) 10-fold cross validation in the training cohort (HN1), and their prognostic performance was evaluated in the validation cohort (HN2) using the area under receiver operator characteristic (ROC) curve (AUC). We used the classifier parameters as defined by earlier studies $(30,33)$. All the classifiers, the corresponding parameters and $\mathrm{R}$ packages are listed in (30).

\section{Analysis}

\section{Prognostic Performance}

To investigate and compare different feature selection and classification methods, we used a similar experimental design as defined earlier for a study in NSCLC study (30). We created a three-dimensional parameter grid for the analysis. For each of the thirteen feature selection methods, we incrementally selected features ranging from 5 up to 50 , with an increment of 5 features $(n=5,10,15,20, \ldots, 50)$. These subsets of selected features were then evaluated by using each of the eleven machine learning classifiers and areas under ROC curves (AUCS).

\section{Stability}

Stability of a feature selection method was assessed using a stability measure defined by Yu et al. (38) under the hard data perturbation settings (39). The stability of a method is quantified as the similarity between the results obtained by the same feature selection method, when applied on the two non-overlapping partitions (of size N/2) of the training cohort (HN1). Similarity between the two resultant feature sets was computed using a weighted bipartite graph and Hungarian algorithm (40) as describe in (30). For each feature selection method, we computed the stability 100 times using a bootstrap approach and reported the median \pm standard deviation (STD) values.

Classifier stability was quantified using the relative standard deviation (RSD \%) and a bootstrap approach. We first selected 30 representative features using the MRMR based feature selection method and used them to compute the classifier stability. We used MRMR because it had highest prognostic performance in all the feature selection methods. For each classification method, we trained the model on the subsampled training cohort (size N/2) and validated the performance on the validation cohort using AUC. Subsampling of the training cohort was done 100 times using a bootstrap approach. RSD is the absolute value of the coefficient of variation and is often expressed in percentage. Here, it was defined as

$$
R S D=\frac{\sigma_{A U C}}{\mu_{A U C}} * 100
$$


where $\sigma_{\mathrm{AUC}}$ and $\mu_{\mathrm{AUC}}$ were the STD and mean of the $100 \mathrm{AUC}$ values, respectively. It should be noted that higher stability in the case of classifiers corresponds to lower RSD values.

\section{Prognostic Performance and Stability}

In order to identify the reliable and accurate methods, we used the median values of AUC and stability as thresholds. We created two rank lists based on AUC \& stability and cited the methods as highly accurate and reliable, which ranked in the top half ( $\geq$ median value) in both ranked lists. Feature selection methods with stability $\geq 0.66$ (median stability of all feature selection methods) and AUC $\geq 0.61$ (median AUC of all feature selection methods) are considered as highly reliable and accurate methods. Similarly, classification methods with RSD $\leq 11.4$ (median RSD of all classifiers) and AUC $\geq 0.61$ (median AUC of all classifiers) are considered as highly reliable and accurate ones.

\section{Experimental Factors Affecting Radiomics Based Survival Prediction}

There are three main experimental factors, which can potentially affect the prediction of radiomics based survival prediction: feature selection method, classification method and the number of selected features. Multifactor ANOVA was used to quantify the variability in AUC scores contributed by these factors and their interactions. In order to compare the variability contributed by each factor, the estimated variance components were divided by the total variance.

\section{Comparison with the NSCLC cohort study}

The results of this study were relatively compared with the previously published study of NSCLC radiomic cohorts (30). For both NSCLC and HNSCC studies, all methods were categorized into two groups: low (< threshold) or high (> threshold). This grouping was carried out using the corresponding threshold values (median AUC and median stability). A method was considered consistent, if it belonged to the same group (high or low) in both studies. It should be noted that that as one feature selection method and one classification method was removed from the analysis for the HNSCC study, they were also not considered while deciding thresholds for NSCLC study. All values of AUC and stability and thresholds along with the group information are reported in Table-2 (feature selection methods) and Table-3 (classification methods).

All analysis was done using R software (R Core Team, Vienna, Austria) version 3.1.2 and Matlab R2012b (The Mathworks, Natick, MA) with Windows 7.

\section{RESULTS}

To assess different machine learning methods for radiomic survival models of head and neck cancer patients, we extracted 440 radiomic features from the segmented tumor 
regions of two independent HNSCC cohorts. Cohort HN1 ( $n=101$ patients) was used for feature selection and classification training, whereas the prediction performance was assessed using the validation cohort HN2 ( $n=95$ patients) [see Figure 1].

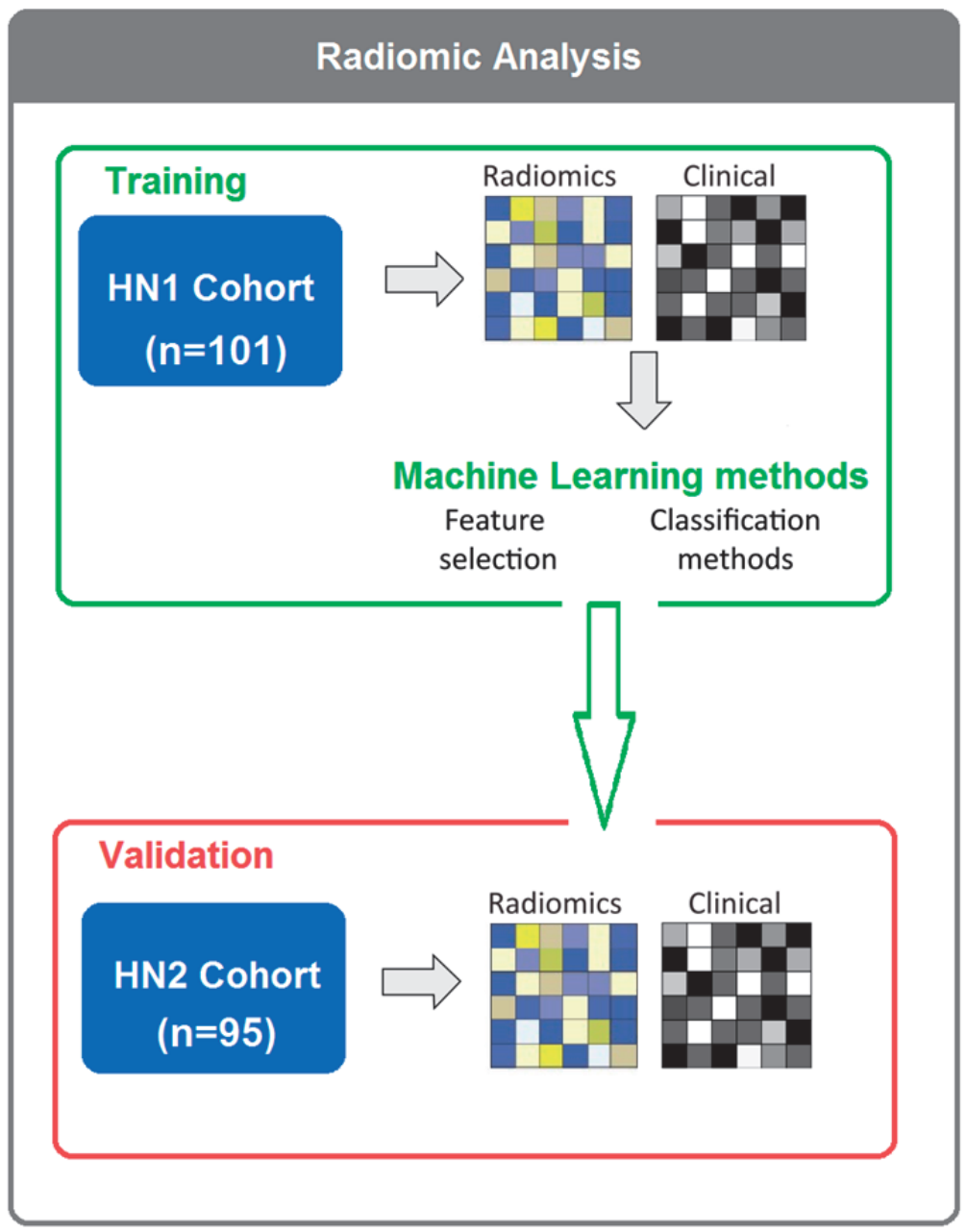

Figure 1. In total 196 HNSCC patients were considered. 440 radiomic features were extracted from the segmented tumor regions of the CT images. Feature selection and classification training was done using the training cohort HN1 $(n=101)$, whereas HN2 $(n=95)$ cohort was used as a validation cohort.

\section{Prognostic Performance}

We used area under receiver operating characteristics curve (AUC) to quantify the prognostic performance of different feature selection and classification methods. Figure 2 depicts the performance of feature selection (in rows) and classification methods (in columns) using the 30 top ranked features after applying feature selection. A median 
AUC of all thirteen feature selection AUC values was used as the representative AUC of a classifier. Similarly, for each feature selection method, a median of eleven classification AUCs was used as the representative AUC. These representative AUC values for the feature selection and classification methods are given in Table 2 and Table 3, respectively.

For feature selection methods, minimum redundancy maximum relevance (MRMR) (AUC: $0.69 \pm 0.07$ ) and mutual information feature selection (MIFS) (AUC: $0.66 \pm 0.07$ ) showed high prognostic performance, whereas the methods WLCX (AUC: $0.55 \pm 0.06$ ) and DISR (AUC: $0.56 \pm 0.06$ ) had lowest median AUCs.

In the case of classification methods, generalized linear models (GLM) (AUC: $0.72 \pm$ 0.08) (median $\pm \mathrm{std}$ ) and partial least squares and principle component regression (PLSR) (AUC: $0.73 \pm 0.07$ ) had highest prognostic performance, whereas bagging (BAG) (AUC: $0.55 \pm 0.06$ ), decision tree (DT) (AUC: $0.56 \pm 0.05$ ) and boosting (BST) (AUC: $0.56 \pm$ $0.07)$ showed lower AUC values. We repeated the above experiment by varying the number of selected features (range 5-50). Results with respect to 10, 20, 40 and 50 representative (top ranked) features are reported in supplement Figures S1, S2, S3 and S4. In addition, median AUC values over each of the experimental factors (feature selection methods, classification methods and number of selected features) are depicted by the heatmaps in supplement Figures S5, S6 and S7. Here as well, GLM and PLSR (classifiers) and MRMR and MIFS (feature selection methods) showed highest median AUCs in majority of cases.

\section{Stability}

To assess the stability of feature selection methods against data perturbation, we used the hard data perturbation setting (39). We observed that WLCX (stability $=0.71 \pm 0.06$ ) (median \pm STD), ICAP (stability $=0.71 \pm 0.03$ ), and CMIM (stability $=0.71 \pm 0.04$ ) showed high stability against data perturbation, whereas FSCR (stability $=0.51 \pm 0.13$ ) and TSCR (stability $=0.54 \pm 0.10$ ) had lower stability [Table 2].

In order to assess the stability of a classifier, we used the relative standard deviation (RSD) and a bootstrap approach. We observed that RF (RSD = 7.36\%) and BAG (9.27\%) were relatively more stable classification methods. PLSR (RSD = $12.75 \%$ ) and SVM (RSD $=12.69 \%$ ) showed higher RSD, which indicated lower stability of these methods. RSD (\%) values corresponding to all eleven classifiers are reported in Table 3. 

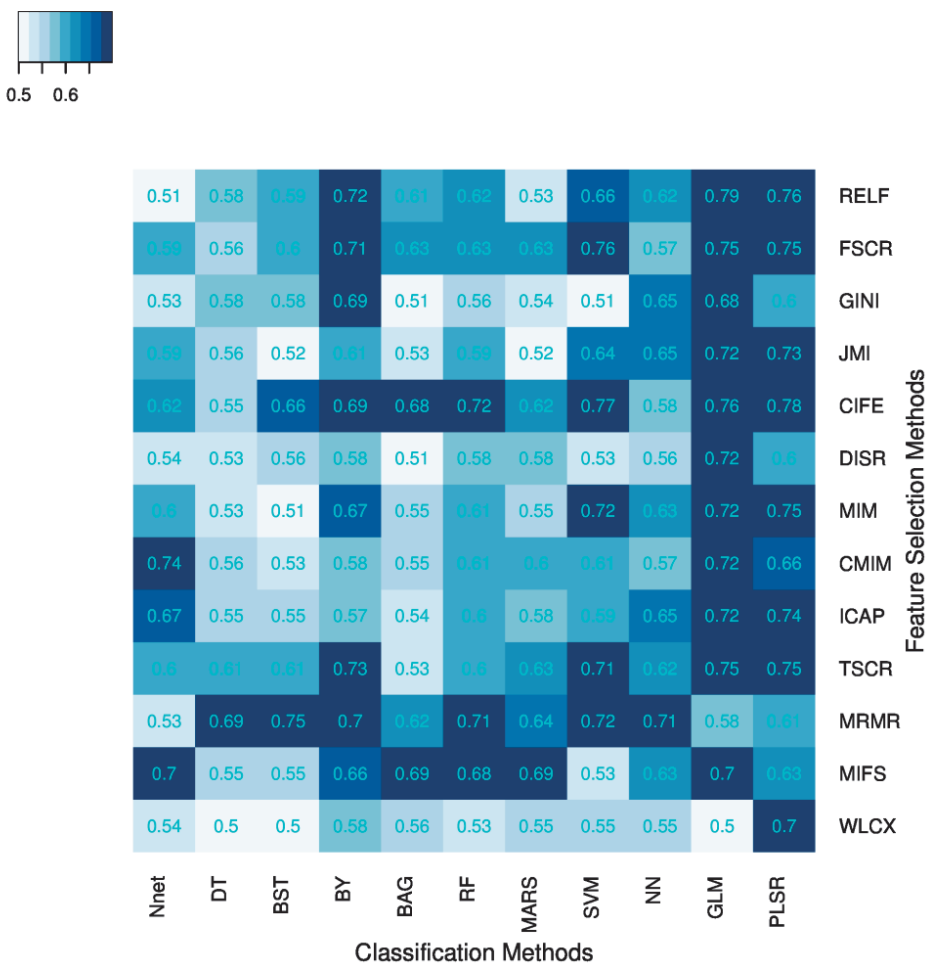

Figure 2. Heatmap depicting the prognostic performance (AUC) of feature selection (in rows) and classification (in columns) methods. It can be observed that PLSR and GLM classification methods and feature selection methods MRMR and MIFS shows relatively high prognostic performance in many cases.

\section{Prognostic Performance and Stability}

Scatterplots in Figure 3 display the stability and prognostic performance of different feature selection and classification methods. It can be observed that the feature selection methods MRMR (AUC $=0.69 \pm 0.07$, stability $=0.66 \pm 0.03$,), MIFS (AUC $=0.66 \pm$ 0.07 , stability $=0.69 \pm 0.04$, ), and CIFE (AUC $=0.68 \pm 0.08$, stability $=0.7 \pm 0.04)$ showed higher prognostic performance and stability than the corresponding median values across all feature selection methods $(A \cup C=0.61$, stability $=0.66)$. Similarly for classification methods, the stability and prognostic performance RF (AUC $=0.61 \pm 0.06, \mathrm{RSD}=$ $7.36 \%$ ), NN (AUC $=0.62 \pm 0.05, \mathrm{RSD}=10.52 \%)$, and $\mathrm{BY}(\mathrm{AUC}=0.67 \pm 0.06, \mathrm{RSD}=$ $11.28 \%$ ) were better than the corresponding median values $(\mathrm{RSD}=11.4 \%, \mathrm{AUC}=0.61)$. 

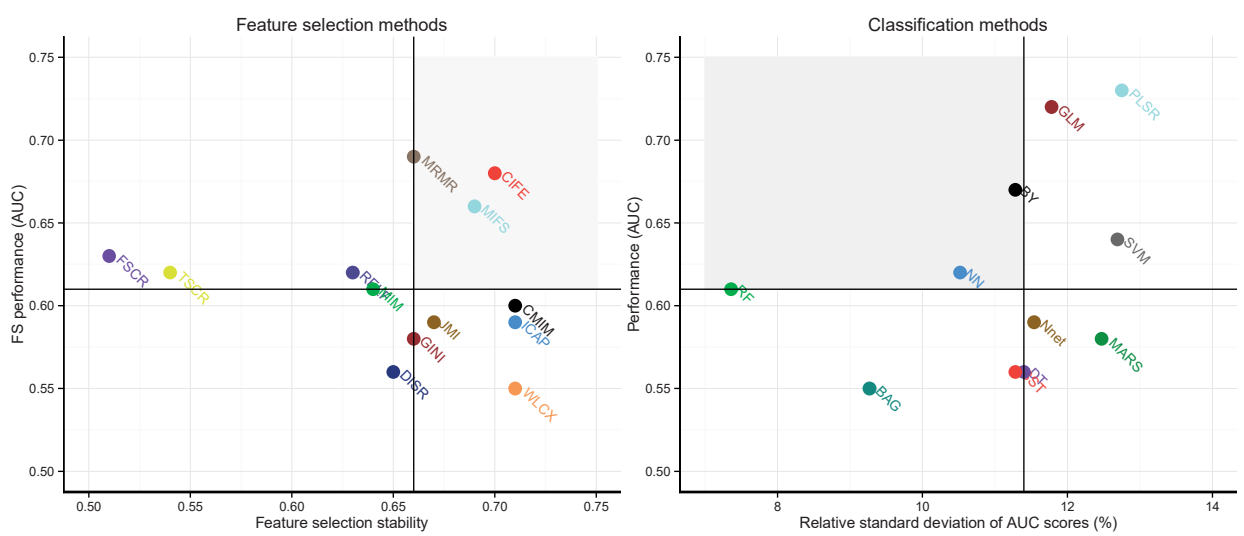

Figure 3. Scatterplots of stability and prognostic performance (AUC) for feature selection (FS) (Left) and classification methods (CF) (right). Feature selection methods having stability $\geq 0.66$ (median stability of FS) and $A \cup C \geq 0.61$ (median AUC of FS) are considered as highly reliable and prognostic methods. Similarly, classification methods having RSD $\leq 11.4$ (median RSD of CF) and AUC $\geq 0.61$ (median AUC of CF) are considered as highly reliable and accurate ones. Highly reliable and prognostic methods are displayed in a gray square region.

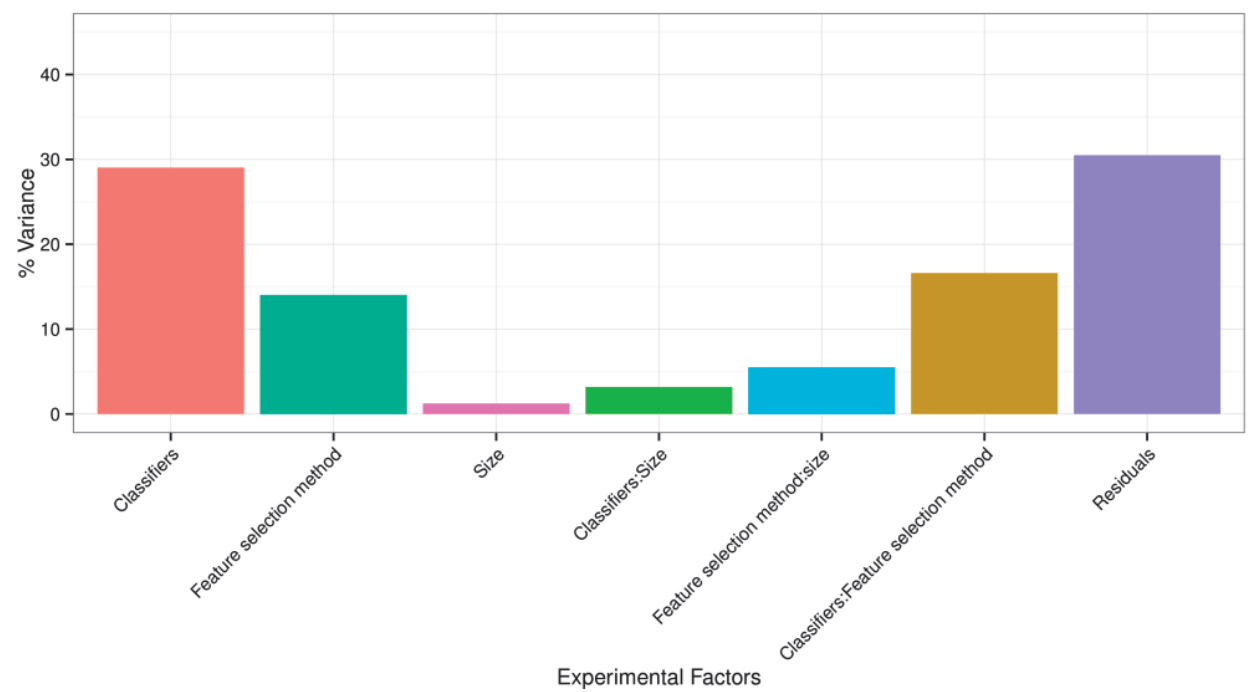

Figure 4. Variation of AUC explained by the experimental factors and their interactions. It can be observed that classification method was the most dominant source of variation in prediction score. Size of the selected (representative) feature subset shared the least of the total variance.

\section{Experimental Factors Affecting Radiomics Based Survival Prediction}

To quantify the effects of the three experimental factors (feature selection methods, classification methods and the number of selected features), we performed multifactor analysis of variance (ANOVA) on AUC scores. We observed that all three experimental parameters are the significant factors affecting the prognostic performance [Figure 4]. 
Classification method was the most dominant source of variability as it explained $29.02 \%$ of the total variance in AUC scores. Feature selection accounted for the $14.02 \%$, whereas interaction of classifier \& feature selection explained $16.59 \%$ of the total variance. Size of the selected (representative) feature subset only shared $1.22 \%$ of the total variance [Figure 4].

\section{Comparison with the NSCLC cohorts}

We compared the obtained results related to feature selection and classification methods to the previously published study of NSCLC cohorts (30) [see Table 2,3].

Two feature selection methods MRMR and MIFS displayed high prognostic performance and stability across both the cancer types. Methods RELF and MIM showed high prognostic power in both the cancer types. However, they had marginally low stability for HNSCC cohorts. Interestingly, WLCX had highest prognostic performance for NSCLC cohorts, whereas it showed lowest prognostic performance for HNSCC cohorts. However, in both the cancer types WLCX displayed high stability against data perturbation [see Table 2].

Three classification methods BY, RF, and NN showed high prognostic performance and stability across both cancer types. PLSR and GLM had high prognostic performance in both cancer types, but these two methods showed low stability for HNSCC cohorts. Classifier displayed lowest prognostic power in HNSCC radiomic cohorts, whereas it had showed the second highest performance in NSCLC cohorts. It should be noted that the stability of BAG was high in both cancer types [see Table 3].

\section{DISCUSSION}

The applications of medical imaging in cancer diagnostics and treatment planning have expanded greatly over the time. Moreover, developments in imaging technologies and computational approaches have led the emergence of "Radiomics", which is a highthroughput medical image data mining field (1). Radiomics is a non-invasive and costeffective medical informatics approach, which provides unprecedented opportunities for improvising clinical decision support (2). Hence, in the context of radiomics, medical imaging is expected to have a more central role in cancer care $(1,2,26)$. With increasing cohort sizes and expanding feature dimensions, radiomics targets a large pool of medical imaging data ("Big data"). Automated, reliable, and efficient methods are desired to extract and mine the most relevant information from these large radiomic cohorts. A recently published study (30) has articulated the scope and applicability of different machine learning methods in two independent radiomic cohorts of patients with NSCLC. They proposed an unbiased framework to compare the prognostic performance and stability of different machine-learning methods. It was recommended that these different machine learning methods should be further evaluated in different cancer types 
and with respect to different radiomic cohorts (30). Furthermore, it has been previously shown that the grouping and prognostic characteristics of radiomic features are cancer specific (16). Therefore, the primary objective of our study was to assess the state of the art machine learning methods in two independent HNSCC cohorts.

Our analysis quantifies the prognostic power and stability of different machine learning methods for the survival prediction of head and neck cancer patients. Depending on the requirement, one may prefer higher prognostic power or stability and choose the methods accordingly. Considering the stability and prognostic performance together, three feature selection methods MRMR, MIFS and CIFE and three classification methods BY, RF and NN should be preferred for head and neck radiomic analyses as they displayed relatively higher prognostic power and stability than other methods.

Assessing the variability in prediction performance by multifactor ANOVA, we found that the classification method is the most dominant source of variation in prediction performance and hence it should be chosen carefully. Size of the selected feature subset contributed the least in the AUC variation.

Comparing the results of this study with the previously published study of NSCLC, we observed that except BAG and MARS, all classifiers showed consistency in their prognostic performance across the two cancer types. However, it should be noted that the AUC values for MARS were quite close to the threshold (median AUC). In the case of feature selection methods, WLCX showed no prediction consistency across the two cancer types. Besides that, methods CIFE, DISR, GINI, CMIM, and ICAP also showed no consistency in the grouping based on prognostic performance. However, it should be noted that for methods CMIM and ICAP, the AUC values did not differ much from the threshold (median AUC). As far as stability is concerned, except for classifiers PLSR, GLM, DT and BST, all classifiers showed consistency in their stability-based grouping. For feature selection methods, only MRMR, MIFS, WLCX and DISR showed consistent stability based grouping across both the studies. It should be noted that for HNSCC cohorts, stability values for all the methods (classification and feature selection) were lower than the ones obtained in NSCLC cohorts. The intuitive explanation for this could be the smaller cohort size. The training cohort in NSCLC study (30) had almost three times more samples than the training cohort of our HNSCC study.

Considering the stability and prognostic performance together and comparing the results between the two cancer types (HNSCC and NSCLC), we observed that the three classifiers BY, RF and NN had high stability and high prognostic performance in both HNSCC and NSCLC studies. Similarly, two feature selection methods MRMR and MIFS showed consistently high values of AUC and stability in both cancer types. These results indicate that such methods should be first preferences for radiomics based prognostic analyses due to their consistency. A note of caution: Different methods are categorized into high/low (prediction performance and stability) group based on simple thresholds (median AUC and median stability). These thresholds are no gold standard and they are only used for comparing the performances of different machine learning methods in a relative manner. It can be observed from the results (Figure-3 and Figure-3 (30)) that 
some of the so-called "not consistent" methods are quite close to the thresholds and they should not be neglected completely. Further validation of these methods with different clinical outcomes, different imaging modalities and also different radiomic cohorts could provide better insights about their applicability.

Results related to the variability of AUC scores were comparable in both the cancer types as in both studies classification method contributed highest and size contributed the least in the performance variance. Interestingly, for HNSCC cohorts, feature selection method contributed almost two times more in the AUC variation than in the case of NSCLC study (30).

As mentioned previously (30), the machine learning methods used in this analysis were chosen because of their simplicity, efficiency and popularity in literature. Furthermore, an interesting discussion about the publicly available implementation tools and the used parameter configurations was presented before (30). It has also been shown that statistical models based on patient's tumor and treatment characteristics provide significantly better predictions/prognosis than the human expert (41). Also, the potential clinical utility of radiomics based prognostic models have been highlighted before (2). Hence, with the expanding radiomic cohorts and feature dimensions, as well as by integrating different biological and clinical information together with radiomics, higher prognostic performance could be achieved. In this regard, our studies could be an important reference as we compared a large panel of machine learning methods across two different cancer types. The prognostic power and stability of different machine learning methods were compared using four independent radiomic cohorts. Such a comparative investigation could help in identifying the optimal and reliable machine learning methods for radiomics based prognostic analyses, which overall could broaden the scope of radiomics in cancer care. 


\section{TABLES}

Table 1. Table defining the acronyms related to the used feature selection and classification methods.

\begin{tabular}{|c|c|c|c|}
\hline $\begin{array}{l}\text { Classification } \\
\text { method acronym }\end{array}$ & Classification method name & $\begin{array}{l}\text { Feature Selection } \\
\text { method acronym }\end{array}$ & $\begin{array}{l}\text { Feature selection method } \\
\text { name }\end{array}$ \\
\hline Nnet & Neural network & RELF & Relief \\
\hline DT & Decision Tree & FSCR & Fisher score \\
\hline BST & Boosting & GINI & Gini index \\
\hline BY & Bayesian & JMI & Joint mutual information \\
\hline BAG & Bagging & CIFE & $\begin{array}{l}\text { Conditional infomax feature } \\
\text { extraction }\end{array}$ \\
\hline RF & Random Forset & DISR & $\begin{array}{l}\text { Double input symmetric } \\
\text { relevance }\end{array}$ \\
\hline MARS & $\begin{array}{l}\text { Multi adaptive regression } \\
\text { splines }\end{array}$ & MIM & $\begin{array}{l}\text { Mutual information } \\
\text { maximization }\end{array}$ \\
\hline SVM & Support vector machines & CMIM & $\begin{array}{l}\text { Conditional mutual information } \\
\text { maximization }\end{array}$ \\
\hline NN & Neirest neighbour & ICAP & Interaction capping \\
\hline GLM & Generalized linear models & TSCR & T-test score \\
\hline PLSR & $\begin{array}{l}\text { Partial least squares and } \\
\text { prinicipal componenet } \\
\text { regression }\end{array}$ & MRMR & $\begin{array}{l}\text { Minimum redundancy } \\
\text { maximum relevance }\end{array}$ \\
\hline- & - & MIFS & $\begin{array}{l}\text { Mutual information feature } \\
\text { selection }\end{array}$ \\
\hline- & - & WLCX & Wilcoxon \\
\hline
\end{tabular}

Table 2. Table describing the representative AUC and stability of feature selection methods. (HNSCC thresholds: $A \cup C=0.61$, Stability $=0.66)($ NSCLC thresholds: $A \cup C=0.61$, Stability $=0.74)$

\begin{tabular}{lllll}
\hline $\begin{array}{l}\text { Feature Selection } \\
\text { method }\end{array}$ & AUC (HNSCC) & AUC (NSCLC) & Stability (HNSCC) & Stability (NSCLC) \\
\hline RELF & $0.62 \pm 0.09$ (High) & $0.61 \pm 0.04$ (High) & $0.63 \pm 0.12$ (Low) & $0.91 \pm 0.05$ (High) \\
FSCR & $0.63 \pm 0.08$ (High) & $0.62 \pm 0.04$ (High) & $0.51 \pm 0.13$ (Low) & $0.78 \pm 0.08$ (High) \\
GINI & $0.58 \pm 0.07$ (Low) & $0.62 \pm 0.04$ (High) & $0.66 \pm 0.11$ (High) & $0.68 \pm 0.10$ (Low) \\
JMI & $0.59 \pm 0.07$ (Low) & $0.61 \pm 0.04$ (High) & $0.67 \pm 0.05$ (High) & $0.68 \pm 0.05$ (Low) \\
CIFE & $0.68 \pm 0.08$ (High) & $0.60 \pm 0.03$ (Low) & $0.7 \pm 0.04$ (High) & $0.69 \pm 0.05$ (Low) \\
DISR & $0.56 \pm 0.06$ (Low) & $0.62 \pm 0.05$ (High) & $0.65 \pm 0.08$ (Low) & $0.69 \pm 0.05$ (Low) \\
MIM & $0.61 \pm 0.08$ (High) & $0.61 \pm 0.04$ (High) & $0.64 \pm 0.1$ (Low) & $0.94 \pm 0.02$ (High) \\
CMIM & $0.6 \pm 0.07$ (Low) & $0.62 \pm 0.04$ (High) & $0.71 \pm 0.04$ (High) & $0.73 \pm 0.04$ (Low) \\
ICAP & $0.59 \pm 0.07$ (Low) & $0.61 \pm 0.03$ (High) & $0.71 \pm 0.03$ (High) & $0.72 \pm 0.04$ (Low) \\
TSCR & $0.62 \pm 0.07$ (High) & $0.61 \pm 0.02$ (High) & $0.54 \pm 0.01$ (Low) & $0.78 \pm 0.12$ (High) \\
MRMR & $0.69 \pm 0.07$ (High) & $0.63 \pm 0.06$ (High) & $0.66 \pm 0.03$ (High) & $0.74 \pm 0.03$ (High) \\
MIFS & $0.66 \pm 0.07$ (High) & $0.63 \pm 0.06$ (High) & $0.69 \pm 0.04$ (High) & $0.8 \pm 0.03$ (High) \\
WLCX & $0.55 \pm 0.06$ (Low) & $0.65 \pm 0.02$ (High) & $0.71 \pm 0.06$ (High) & $0.84 \pm 0.05$ (High) \\
\hline
\end{tabular}


Table 3. Table describing the representative AUC and stability of classification methods. (HNSCC thresholds: AUC =0.61, RSD \% = 11.4) (NSCLC thresholds: $A \cup C=0.61$, RSD \% = 5.56)

\begin{tabular}{lllll}
\hline Classification method AUC (HNSCC) & AUC(NSCLC) & RSD \% (HNSCC) & RSD \% (NSCLC) \\
\hline Nnet & $0.59 \pm 0.07$ (Low) & $0.57 \pm 0.04$ (Low) & 11.54 (Low) & 6.41 (Low) \\
DT & $0.56 \pm 0.05$ (Low) & $0.54 \pm 0.04$ (Low) & 11.4 (High) & 7.89 (Low) \\
BST & $0.56 \pm 0.07$ (Low) & $0.58 \pm 0.04$ (Low) & 11.28 (High) & 8.23 (Low) \\
BY & $0.67 \pm 0.06$ (High) & $0.64 \pm 0.05$ (High) & 11.28 (High) & 0.86 (High) \\
BAG & $0.55 \pm 0.06$ (Low) & $0.64 \pm 0.03$ (High) & 9.27 (High) & 5.56 (High) \\
RF & $0.61 \pm 0.06$ (High) & $0.66 \pm 0.03$ (High) & 7.36 (High) & 3.52 (High) \\
MARS & $0.58 \pm 0.05$ (Low) & $0.61 \pm 0.03$ (High) & 12.47 (Low) & 6.98 (Low) \\
SVM & $0.64 \pm 0.09$ (High) & $0.61 \pm 0.03$ (High) & 12.69 (Low) & 6.39 (Low) \\
NN & $0.62 \pm 0.05$ (High) & $0.61 \pm 0.02$ (High) & 10.52 (High) & 4.08 (High) \\
GLM & $0.72 \pm 0.08$ (High) & $0.63 \pm 0.02$ (High) & 11.78 (Low) & 2.19 (High) \\
PLSR & $0.73 \pm 0.07$ (High) & $0.63 \pm 0.02$ (High) & 12.75 (Low) & 2.24 (High) \\
\hline
\end{tabular}

\section{ACKNOWLEDGMENTS}

Authors acknowledge financial support from the National Institute of Health (NIH-USA U24CA194354 and NIH-USA U01CA190234). This research is also supported by the Dutch technology Foundation STW (grant n 10696 DuCAT). Authors also acknowledge financial support from EU 7th framework program (ARTFORCE - $n^{\circ} 257144$, REQUITE $n^{\circ}$ 601826), SME Phase 2 (EU proposal 673780 - RAIL), the European Program H2020PHC-2015 (BD2decide, n²10274050), Alpe d’HuZes-KWF (DESIGN).

\section{SUPPLEMENTARY INFORMATION}

https://www.ncbi.nIm.nih.gov/pmc/articles/PMC4668290/bin/Data_Sheet_1.DOCX 


\section{REFERENCES}

1. Lambin P, Rios-Velazquez E, Leijenaar R, Carvalho S, van Stiphout RG, Granton P, et al. Radiomics: extracting more information from medical images using advanced feature analysis. European Journal of Cancer. 2012;48(4):441-6.

2. Aerts HJ, Velazquez ER, Leijenaar RT, Parmar C, Grossmann P, Carvalho S, et al. Decoding tumour phenotype by noninvasive imaging using a quantitative radiomics approach. Nature communications. 2014;5.

3. Fisher R, Pusztai L, Swanton C. Cancer heterogeneity: implications for targeted therapeutics. British journal of cancer. 2013;108(3):479-85.

4. Ng CK, Pemberton HN, Reis-Filho JS. Breast cancer intratumor genetic heterogeneity: causes and implications. 2012.

5. Szerlip NJ, Pedraza A, Chakravarty D, Azim M, McGuire J, Fang Y, et al. Intratumoral heterogeneity of receptor tyrosine kinases EGFR and PDGFRA amplification in glioblastoma defines subpopulations with distinct growth factor response. Proceedings of the National Academy of Sciences. 2012;109(8):3041-6.

6. Longo DL. Tumor heterogeneity and personalized medicine. N Engl J Med. 2012;366(10):956-7.

7. Alic L, Niessen WJ, Veenland JF. Quantification of heterogeneity as a biomarker in tumor imaging: a systematic review. PloS one. 2014;9(10):e110300.

8. Carvalho S, Leijenaar RT, Velazquez ER, Oberije C, Parmar C, Van Elmpt W, et al. Prognostic value of metabolic metrics extracted from baseline positron emission tomography images in non-small cell lung cancer. Acta Oncologica. 2013;52(7):1398-404.

9. Gevaert O, Mitchell LA, Achrol AS, Xu J, Echegaray S, Steinberg GK, et al. Glioblastoma multiforme: exploratory radiogenomic analysis by using quantitative image features. Radiology. 2014;273(1):168-74.

10. Hatt M, Tixier F, Le Rest CC, Pradier O, Visvikis D. Robustness of intratumour 18F-FDG PET uptake heterogeneity quantification for therapy response prediction in oesophageal carcinoma. European journal of nuclear medicine and molecular imaging. 2013;40(11):1662-71.

11. Jain R, Poisson LM, Gutman D, Scarpace L, Hwang SN, Holder CA, et al. Outcome prediction in patients with glioblastoma by using imaging, clinical, and genomic biomarkers: focus on the nonenhancing component of the tumor. Radiology. 2014;272(2):484-93.

12. Leijenaar RT, Carvalho S, Velazquez ER, Van Elmpt WJ, Parmar C, Hoekstra OS, et al. Stability of FDG-PET Radiomics features: An integrated analysis of test-retest and inter-observer variability. Acta Oncologica. 2013;52(7):1391-7.

13. Parmar C, Velazquez ER, Leijenaar R, Jermoumi M, Carvalho S, Mak RH, et al. Robust radiomics feature quantification using semiautomatic volumetric segmentation. 2014.

14. Hunter LA, Krafft S, Stingo F, Choi H, Martel MK, Kry SF. High quality machine-robust image features: Identification in nonsmall cell lung cancer computed tomography images. Medical physics. 2013;40(12):121916.

15. Basu S, Hall LO, Goldgof DB, Gu Y, Kumar V, Choi J, et al., editors. Developing a classifier model for lung tumors in CT-scan images. Systems, Man, and Cybernetics (SMC), 2011 IEEE International Conference on; 2011: IEEE.

16. Parmar C, Leijenaar RT, Grossmann P, Velazquez ER, Bussink J, Rietveld D, et al. Radiomic feature clusters and Prognostic Signatures specific for Lung and Head \& Neck cancer. Scientific reports. 2015;5.

17. Ganeshan B, Goh V, Mandeville HC, Ng QS, Hoskin PJ, Miles KA. Non-small cell lung cancer: histopathologic correlates for texture parameters at CT. Radiology. 2013;266(1):326-36.

18. Cistaro A, Quartuccio N, Mojtahedi A, Fania P, Filosso PL, Campenni A, et al. Prediction of 2 years-survival in patients with stage I and II non-small cell lung cancer utilizing 18F-FDG PET/CT SUV quantifica. Radiology and oncology. 2013;47(3):219-23.

19. Cook GJ, Yip C, Siddique M, Goh V, Chicklore S, Roy A, et al. Are Pretreatment 18F-FDG PET Tumor Textural Features in Non-Small Cell Lung Cancer Associated with Response and Survival After Chemoradiotherapy? Journal of Nuclear Medicine. 2013;54(1):19-26.

20. Hawkins SH, Korecki JN, Balagurunathan Y, Gu Y, Kumar V, Basu S, et al. Predicting Outcomes of Nonsmall Cell Lung Cancer Using CT Image Features. 
21. Ganeshan B, Abaleke S, Young RC, Chatwin CR, Miles KA. Texture analysis of non-small cell lung cancer on unenhanced computed tomography: initial evidence for a relationship with tumour glucose metabolism and stage. Cancer Imaging. 2010;10(1):137.

22. Coroller TP, Grossmann P, Hou Y, Velazquez ER, Leijenaar RT, Hermann G, et al. CT-based radiomic signature predicts distant metastasis in lung adenocarcinoma. Radiotherapy and Oncology. 2015.

23. Pickles MD, Manton DJ, Lowry M, Turnbull LW. Prognostic value of pre-treatment DCE-MRI parameters in predicting disease free and overall survival for breast cancer patients undergoing neoadjuvant chemotherapy. European journal of radiology. 2009;71(3):498-505.

24. Segal E, Sirlin CB, Ooi C, Adler AS, Gollub J, Chen X, et al. Decoding global gene expression programs in liver cancer by noninvasive imaging. Nature biotechnology. 2007;25(6):675-80.

25. Nicolasjilwan M, Hu Y, Yan C, Meerzaman D, Holder CA, Gutman D, et al. Addition of MR imaging features and genetic biomarkers strengthens glioblastoma survival prediction in TCGA patients. Journal of Neuroradiology. 2014.

26. Lambin $\mathrm{P}$, van Stiphout RG, Starmans MH, Rios-Velazquez E, Nalbantov G, Aerts HJ, et al. Predicting outcomes in radiation oncology-multifactorial decision support systems. Nature Reviews Clinical Oncology. 2013;10(1):27-40.

27. Kumar V, Gu Y, Basu S, Berglund A, Eschrich SA, Schabath MB, et al. Radiomics: the process and the challenges. Magnetic resonance imaging. 2012;30(9):1234-48.

28. Mohri M, Rostamizadeh A, Talwalkar A. Foundations of machine learning: MIT press; 2012.

29. Guyon I, Elisseeff A. An introduction to variable and feature selection. The Journal of Machine Learning Research. 2003;3:1157-82

30. Parmar C, Grossmann P, Bussink J, Lambin P, Aerts HJ. Machine Learning methods for Quantitative Radiomic Biomarkers. Scientific reports. 2015;5.

31. Brown G, Pocock A, Zhao M-J, Luján M. Conditional likelihood maximisation: a unifying framework for information theoretic feature selection. The Journal of Machine Learning Research. 2012;13(1):27-66.

32. Zhao Z, Morstatter F, Sharma S, Alelyani S, Anand A, Liu H. Advancing feature selection research. ASU feature selection repository. 2010.

33. Fernández-Delgado M, Cernadas E, Barro S, Amorim D. Do we need hundreds of classifiers to solve real world classification problems? The Journal of Machine Learning Research. 2014;15(1):3133-81.

34. Haralick RM, Shanmugam K, Dinstein IH. Textural features for image classification. Systems, Man and Cybernetics, IEEE Transactions on. 1973(6):610-21.

35. Galloway MM. Texture analysis using gray level run lengths. Computer graphics and image processing. $1975 ; 4(2): 172-9$

36. Deasy JO, Blanco Al, Clark VH. CERR: a computational environment for radiotherapy research. Medical physics. 2003;30(5):979-85.

37. Kuhn M. Building predictive models in $\mathrm{R}$ using the caret package. Journal of Statistical Software. 2008;28(5):1-26.

38. Yu L, Ding C, Loscalzo S, editors. Stable feature selection via dense feature groups. Proceedings of the 14th ACM SIGKDD international conference on Knowledge discovery and data mining; 2008: ACM.

39. Haury A-C, Gestraud P, Vert J-P. The influence of feature selection methods on accuracy, stability and interpretability of molecular signatures. PloS one. 2011;6(12):e28210.

40. Kuhn HW. The Hungarian method for the assignment problem. Naval research logistics quarterly. 1955;2(1-2):83-97.

41. Oberije C, Nalbantov G, Dekker A, Boersma L, Borger J, Reymen B, et al. A prospective study comparing the predictions of doctors versus models for treatment outcome of lung cancer patients: a step toward individualized care and shared decision making. Radiotherapy and Oncology. 2014;112(1):37-43. 
Chapter

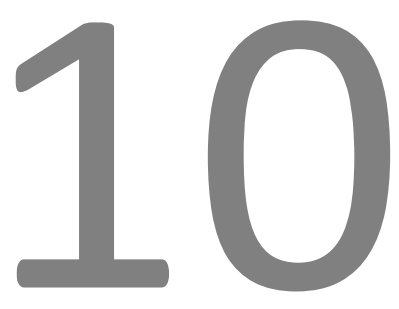

General discussion and future perspectives 



\section{DISCUSSION}

In the past decade, increasing evidences have been observed suggesting the genetic ${ }^{1}$, epigenetic $^{2}$, transcriptomic ${ }^{3}$, histological ${ }^{4}$ and micro-environmental ${ }^{5}$ heterogeneity of individual tumors ${ }^{6}$. Often, these intra- and inter-tumor heterogeneities cause the differences in treatment response of the patients with same diagnosis. Several new diagnostic techniques and treatment modalities have helped the major advances in cancer care. Developments related to the information and communication technologies and computational sciences have increased our ability to collect, store, share, analyze and interpret biomedical data, which has led the emergence of precision oncology-practice of tailoring treatment strategies to individual patients/tumors. There are several different ways to characterize tumors, for example, with non-invasive imaging, or invasively with blood samples and tissue biopsies from which a number of molecular quantities can be measured. The focus of this thesis is on "radiomics", which is a non-invasive medical imaging based quantitative approach towards precision oncology. Chapter-1 introduced radiomics from the precision oncology perspective along with the overall workflow of the process and the thesis outline. It also articulated some of the successful applications and associated challenges.

\section{ASSESSING VARIABILITY IN RADIOMICS}

The first part of the thesis focused on evaluating different publicly available semiautomatic segmentation methods keeping in mind their applicability for raidomics. Two different semi-automatic segmentation approaches available in a free and publicly available 3D-Slicer software tool, were compared to the manual segmentations in terms of their efficiency and accuracy. Moreover, reproducibility of radiomic features against different segmentation approaches was evaluated. These investigations could help in achieving higher efficiency and robustness for radiomic analyses.

\section{Semiautomatic segmentations for quantitative imaging}

Despite the efforts in standardizing protocols of medical imaging and tumor delineation, target definition remains subjected to observer variation. With respect to manual delineations, the addition of PET information to CT imaging in standardized delineation protocols has reduced the observer variability, however, human interaction and interpretation of medical images is still a considerable source of variation $7,8,9$. In Chapter-2, we evaluated the utility of a freely accessible algorithm, a cellular automaton-based region growing algorithm implemented in 3D-Slicer, by performing a volumetric comparison with tumor delineations made by five independent oncologists following standardized protocols ${ }^{10}$, as well as by comparing it with the maximal diameter obtained from pathological measurements. The volumetric comparison showed that the 3D-Slicer algorithm 
provides tumour segmentations, statistically equivalent to physicians CT/PET manual contours and it takes significantly lower time. Importantly, semi-automatic segmentations showed overall lower volume variability $(p=0.0003)$ and smaller uncertainty areas ( $p=0.0002$ ) compared to manual delineations. 3D-Slicer segmentations showed robustness towards user initialization. Additionally, we observed a strong correlation between the 3D-Slicer segmentations and the maximal diameter as measured on pathological examination $(r=0.89 ; 95 \% \mathrm{Cl}, 0.81-0.94)$. Since the semi-automatic segmentations are statistically comparable to manual delineations and correlated well with pathology, they could be used as a starting point for treatment planning delineations and in high-throughput data mining research, such as Radiomics, where manual tumour delineations are often not available, or represent a time consuming bottleneck. In order to assess the effect of tumor delineations on radiomic features, in Chapter-4, we investigated the robustness of quantitative imaging features, extracted from 3D-Slicer tumor segmentations, as compared to those, extracted from manual tumor delineations. Overall 3D-Slicer based semiautomatic segmentation method produced more reproducible radiomic features $(p=0.0009)$. We also analyzed different feature groups for their reproducibility, and observed that the difference in reproducibility, for intensity statistics and textural features, was statistically significant $(p=0.0006, p=0.0094$, respectively) between the two segmentation strategies. The shape features however did not significantly differ in reproducibility between the two strategies $(p=0.31)$. We also analyzed intra- and inter-observer reproducibility for 3D-Slicer based semiautomatic segmentations. Three independent observers segmented each tumor twice, with different algorithmic initialization. Image descriptors demonstrated high intra-observer reproducibility for 3D-Slicer segmentations, which indicates their robustness over different seed point initializations. We also observed high inter-observer reproducibility in image descriptors for semiautomatic segmentations.

Next, We evaluated a level-set based semi-automatic segmentation algorithm from the chest-imaging platform of 3D-Slicer in Chapter-3. The results were consistent to Chapter-2 although applied on a cohort of large number $(n=354)$ of nodules. Moreover, this study focused on lung screening patient population and low-dose CT images. Semiautomatic segmentations were significantly more stable than the manual delineations. Moreover, level-set based segmentation method was performed using just a single seed point and hence it required minimal manual interaction and was very efficient. Efficient segmentation tool with minimal manual interaction could be a perfect application for screening trials. Often, screening trials include large patient cohorts and many small nodules (abnormalities) are detected during screening. Therefore, an efficient segmentation tool could reduce the overall turnaround time for the nodule delineation and characterization process.

Despite achieving better time efficiency and robustness in many cases, there is still a provision for manual supervision in semi-automatic delineation process. In some exceptional cases, the algorithmic approach underperforms. For example, both semiautomatic approaches failed in delineating extremely large or small masses with pleural 
attachments. Moreover, it is well known that semi-automatic approaches does not work well with the non- and part-solid nodules having hazy appearance and fuzzy boundaries $^{11}, 12,1314$. Therefore, in these cases, the knowledge of experienced radiologists is needed. Several segmentation algorithms have been proposed to improve the contours of structures with hazy appearances, such as the Markov random field theory-based algorithms ${ }^{1115}$ and a hybrid algorithms that combines threshold-based, region growing, connected component analyses and convex hull calculations ${ }^{16}, 11,12,14$, However, these more sophisticated algorithms are not easily accessible and freely available in open source software tools. Furthermore, this cascaded integrator of different algorithms could significantly hamper the time efficiency. Therefore, for quantitative imaging, semi-automatic segmentations followed by manual editing could be a better alternative providing more accurate delineations in an efficient manner. However, these choices obviously could vary depending upon the applications.

\section{MACHINE LEARNING METHODS FOR RADIOMICS}

The second part of the thesis covered the radiomics based predictive/prognostic analyses, where different machine learning approaches of feature selection and modeling were investigated for the radiomic cohorts of lung and/or head \& neck cancer patients.

\section{Radiomics decodes tumor phenotype and demonstrates transferable prognostic power}

In Chapter-5, we explored 440 radiomic features in 1019 cancer patients demonstrating that quantitative imaging biomarkers have strong prognostic performance in large independent cohorts of lung and head and neck cancer, and are associated with the underlying gene expression patterns. Moreover, in this study we selected radiomic features using their reproducibility against manual delineations and test-retest image scans. We also observed that features having high reproducibility have strong prognostic power. Furthermore, we showed the complementary and improved performance of radiomic features compared to TNM staging for prediction of outcome, which illustrates the clinical importance of our findings as TNM is routinely used in the clinic. It is expected that radiomics will have an impact in personalized medicine, as it provides complementary phenotypic information compared to genomics. The results presented in Chapter-5, indicate that radiomic features decode a prognostic phenotype that could be translated from lung to head and neck cancer, which may also generalize to other cancer types. These results indicate that radiomics quantifies a general prognostic cancer phenotype that likely can broadly be applied to other cancer types. Finally, due to the large application of non-invasive imaging in cancer patients in almost all hospitals worldwide, the results of this work would stimulate the development of image-based biomarkers and their evaluation in retrospective and prospective studies ${ }^{17}, 18,19$. 


\section{Cancer-specific grouping and prognostic patters of radiomic predictors}

Although Chapter-5 demonstrated the transferable prognostic abilities of radiomic predictors between cancer types, we carried out the cancer specific radiomic investigations in Chapter- 6 and compared radiomic patterns of lung and head \& neck cancer patients. In this study we reduced the high dimensional radiomic feature space in an unsupervised manner and investigated common and cancer-type specific radiomic patterns. We applied consensus clustering on 440 radiomic features extracted from lung cancer and head \& neck cancer patient cohorts. Furthermore, these clusters were externally validated on independent validation cohorts and were investigated in terms of their association with clinical variables like survival, histology, stage etc. For both cancer types, many clusters showed high cluster consensus and high within cluster correlation, which indicated the high robustness (stability) and compactness of these clusters. Comparing the individual lung and head \& neck feature clusters, we observed that five cluster pairs had substantial overlap (Jaccard $\geq 0.6$ ) between the lung and head \& neck cancer, whereas the overlap for other cluster pairs was poor. These results demonstrate both common as well as cancer-specific clustering characteristics of radiomic features. It can be observed from our analysis that radiomic features also have cancer-specific prognostic ability. We compared the univariable prognostic power of radiomic features across the two cancer types and observed that several radiomic features have significant prognostic utility in only one of the two cancer types. Furthermore the multivariable radiomic signatures performed better in validation cohorts of the same cancer type in our multivariable analysis. The primary objective of our study was to separately investigate and compare radiomic feature subgroups in lung and head \& neck cancer. Our analysis revealed a cancer-specific grouping and prognostic trends of radiomic features, which could be exploited to improve the performance of prognostic models.

\section{Machine learning approaches of feature selection and classification for radiomics}

"Machine-learning" can be broadly defined as computational methods/models which can use experience (data) to make accurate predictions ${ }^{20}$. These programmable computational methods are capable of "learning" from data and hence can automate and improve the prediction process. Predictive and prognostic models with high accuracy, reliability, and efficiency are vital factors driving the success of radiomics. Therefore, it is essential to compare different machine learning models for radiomics based clinical biomarkers. Like any high-throughput data-mining field, radiomics also underlies the curse of dimensionality ${ }^{21}$, which should be addressed by appropriate feature selection strategies. Moreover, feature selection also helps in reducing overfitting of models (increasing the generalizability). Thus, in order to reduce the dimensionality of radiomic feature space and enhance the performance of radiomics based predictive models, different feature selection methods ${ }^{22}$ should be thoroughly investigated. However, as 
radiomics is an emerging research field, most of the published studies have only assessed the predictive capabilities of radiomic features without putting much emphasis on the comparison of different feature selection and predictive modeling methods.

We investigated different machine learning approaches of feature selection and classification for radiomic analyses in Chapters-7, 8, and 9. In an exploratory analysis of chapter-7, we built prediction models for predicting lung cancer histological subtypes. It is estimated that the error rate of cancer histopathology can be as high as $23 \%{ }^{23},{ }^{24},{ }^{2}, 26$. These errors could be due to both sampling errors and observer variability; thus, there is a great need for additional quantitative diagnostic information. In more elaborate and systematic analyses investigating a large panel of machine learning approaches for radiomics based survival prediction in independent cohorts of Lung cancer (Chapter-8) and Head \& Neck cancer (Chapter-9). We evaluated fourteen feature selection methods and twelve classification methods in terms of their predictive performance and stability against data perturbation.

Various feature selection methods have been employed for high-throughput data mining problems ${ }^{27}$. In general, feature selection methods are categorized into three main categories: (1) filter methods (2) wrapper methods and (3) embedded methods. In these studies, we investigated fourteen different filter based approaches for radiomics based survival prediction. We only used filter-based approaches because they are computationally more efficient and less prone to overfitting than the wrapper and embedded methods ${ }^{22}, 28$. Furthermore, unlike wrapper and embedded methods, filter methods are classifier independent. Thus, they allow separation of the modeling and feature selection component of the predictive analysis, which increases the generalizability of each component and hence the overall analysis. We also investigated twelve machinelearning classification methods belonging to twelve different classifier families. Many classifiers have been proposed in the machine-learning literature. Theoretically speaking, these classifiers belong to different fields (classifier families) of computer science and statistics. Therefore, it could really be difficult to understand the underlying assumptions of each and every classifier and tune the parameters in an unbiased manner. The parameter tuning could be biased by user's more (or lack of) expertise with some classifiers over the others. Usually, the studies, which propose a new classifier, only compare it to the reference classifiers of same family excluding the other classifier families. Even if classifiers belonging to different families are considered for comparison, these reference classifiers are usually implemented using simple tools and with limited parameter configurations while carefully tuning the proposed classifier. These could consequently bias the results in favor of the proposed classifiers ${ }^{29}$. In our studies, we did not propose any new classifier and we have used the same implementation tool ( $R$ package caret) for all the classifiers. Furthermore, to ensure unbiased usage of classifiers, we used parameter configurations that were previously reported ${ }^{29}$, in an study of comparing many classifiers over a large number of different datasets exhaustively. These parameter configurations were selected from the literature and have been previously validated on a large number of datasets belonging to different fields. Further- 
more, in our studies, the parameters were tuned using the repeated cross validation of training data only. Hence, our experimental design allowed us to evaluate different classification methods in an unbiased manner.

Considering the stability and prognostic performance together and comparing the results between the two cancer types (HNSCC and NSCLC), we observed that the three classifiers naïve bayes (BY), random forest (RF) and nearest neighbor (NN) had high stability and high prognostic performance in both HNSCC and NSCLC studies. Similarly, two feature selection methods minimum redundancy maximum relevance (MRMR) and mutual information feature selection (MIFS) showed consistently high values of AUC and stability in both cancer types. These results indicate that such methods should be the first preferences for radiomics based prognostic/predictive analyses due to their consistency. However, further validation of these methods with different clinical outcomes, different imaging modalities and also different radiomic cohorts could provide better insights about their applicability.

Results related to the variability of AUC scores were comparable in both the cancer types as in both studies classification method contributed highest and size contributed the least in the performance variance. Interestingly, for HNSCC cohorts (Chapter-9), feature selection method contributed almost two times more in the AUC variation than in the case of NSCLC study of Chapter-8.

It has also been shown that statistical models based on patient's tumor and treatment characteristics provide significantly better predictions/prognosis than the human expert $^{30}$. Also, the potential clinical utility of radiomics based prognostic models has been highlighted before ${ }^{6}$. Hence, with the expanding radiomic cohorts and feature dimensions, as well as by integrating different biological and clinical information together with radiomics, higher prognostic performance could be achieved. In this regard, our studies could be an important reference as we compared a large panel of machine learning methods across two different cancer types. The prognostic power and stability of different machine learning methods were compared using four independent radiomic cohorts. Such comparative investigations could help in identifying the optimal and reliable machine learning methods for radiomics based prognostic analyses, which overall could broaden the scope of radiomics in cancer care.

\section{FUTURE PERSPECTIVES}

Standardization is a vital factor for the pragmatic realization of precision oncology. With respect to quantitative imaging and radiomics, majority of the published analyses have employed retrospective imaging data. In routine clinical image acquisition, there is a wide variation in imaging parameters such as image resolution, slice thickness, reconstruction parameters, imaging scanners etc. In addition to that, as radiomic features are extracted from the tumor regions on images, inter-observer delineation variation could influence the reproducibility of radiomic features. Sensitivity of radiomic predictors with 
respect to these different imaging and clinical settings should be investigated. In this thesis, we investigated the reproducibility of radiomic features against different segmentation methods as well as test-retest image scans. Further such investigations assessing the sensitivity of radiomic predictors with respect to the variation in different imaging settings are required in order to decide standardization protocols. Moreover, standardization of data (imaging and clinical) through the application of protocols and semantic-interoperability across different medical centres and the creation of a framework for reproducible data sharing and research is needed. Locally this can be started with centralizing and making transparent all data and methodologies involved in radiomic analyses. This also will allow the re-use of data for validation purposes or for the analysis of new hypothesis ${ }^{31}$. Several initiatives like TCIA (The Cancer Imaging Archive) ${ }^{32}$ and QIN (Quantitative imaging network) ${ }^{33}$ are already a step further in this regard. These efforts will allow external validation of predictive algorithms and facilitate the reproducibility and the evaluation of new research questions. Infrastructures like euroCAT, could be a crucial step for quality assurance of data collection and standardization, which is the semantic interoperability through different centers and the automated storage of patient, tumor and treatment characteristics. In future, this could address the drawbacks inherent to retrospective data collection and analysis.

Many different types of radiomic features have been defined in the literature ${ }^{34}$ based on tumor intensity histograms, shape and size, texture patterns, descriptors of tumor location and surrounding tissues, as well as the properties of tumor regions in different image transformed domains. These different types of features are extracted using different extraction settings. For example, in order to quantify grey level cooccurrence based textural features, parameters like directions, intensity quantization etc. need to be chosen ${ }^{35}$. Similarly, as far as image transformations are concerned, there are several choices available in the image processing literature ${ }^{36,37}$. Currently, there is a lack of standardization in the feature extraction process. Different research groups use different settings to extract these radiomic features based on their experience and familiarity with these features. Investigations assessing the sensitivity of these different extraction settings are therefore needed in order to standardize the feature extraction tools. Moreover, how these extraction settings could affect the predictive/prognostic performance, is also yet to be investigated. An exhaustive investigation, assessing these extraction settings in different retrospective radiomic cohorts and with respect to different clinical outcomes, could help in standardizing feature extraction in quantitative imaging. Once the extraction method is standardized, open source feature extraction tools could be shared publicly for academic research and that could improve the reproducibility and validity of radiomic research.

Also, there is a need to understand the biological interpretations of these features. In order to widen the scope of radiomics based decision support systems, it is essential to have a deep understanding of these features and their relation with the underlying biology. These kind of semantic investigations could result in enhancing acceptability and validity of these data driven approaches. 
The radiomic feature definitions are mainly taken from the rich knowledgebase of image processing and computer vision, where these features are often referred as handcrafted or hand-designed features. In the recent times, with increasing data and computation power, there has been an emergence of deep learning or unsupervised feature learning. Deep learning is considered as state of the art machine-learning subfield, which has demonstrated excellent performance for several image recognition and computer vision challenges ${ }^{38}$. In medical image analysis as well, several recently published articles demonstrate the unprecedented opportunities of deep learning based applications $^{39,40,41}$. In deep learning, raw data (images for the scope of this thesis) is supplied as input to the deep neural network instead of any handcrafted features. It is expected that with sufficient amount of data, the network itself will learn the required features, which could provide higher predictive/prognostic performance. These kinds of approaches could address all the drawbacks related to feature extraction setting standardization as well as feature selection/reduction. However, there are some inherent challenges. These approaches require a large amount of data and high computation power. Often, in disease specific biomedical research, relatively limited amount of data is available. Therefore, sharing of data and computational resources between different medical centers could help in making such applications feasible for cancer care. Moreover, a large number of hyper parameters need to be chosen in order to train these deep networks and the output could be sensitive to these hyper parameters. Despite these challenges, the demonstrated success of deep learning in computer vision and image recognition as well as their ability to learn the required feature automatically and therefore reducing the manual interaction in decision support, make them worth investigating for radiomics.

The second part of the thesis investigated the performance and robustness of different machine learning approaches for radiomics. In order to avoid overfitting, these investigations were carried out using independent radiomic cohorts. Furthermore, the evaluation framework was unbiased as it used publicly available implementation tools and reported parameter settings. The results of these investigations should be further validated with different radiomic cohorts focusing on different cancer types, different clinical endpoints and different imaging modalities. Moreover, in order to achieve higher predictive/prognostic power, other state of the art machine learning methods should also be incorporated in future investigations. Bias-variance tradeoff is a well-known challenge for machine learning applications ${ }^{20}$. Radiomics based machine learning models should be extensively validated in many different external cohorts. Although, the work done in this thesis used couple of external validation cohorts, a meta analysis, consisting several multicentre radiomic cohorts and different clinical outcomes could provide more insights regarding the actual prediction/prognostic power and generalizability of a models as well as their stability and consistency. However, ethical and privacy concerns, could hinder sharing of medical data between different centers. Therefore, shared data repositories like TCIA and QIN should be appreciated and more centers should participate in such academic and scientific collaborations. Also, other emerging 
alternatives like distributed learning ${ }^{42}$ could also help in external validation of models. However, more research in these areas is required in order to realize their applications for radiomics and precision oncology.

In conclusion, the work done in this thesis indicate non-invasive medical imaging based radiomic predictors could be vital for precision oncology by improving the hospital costs, productivity and quality of cancer care. 


\section{REFERENCES}

1. Gerlinger, M. et al. Intratumor heterogeneity and branched evolution revealed by multiregion sequencing. N. Engl. J. Med. 366, 883-892 (2012).

2. Alizadeh, A. A. et al. Toward understanding and exploiting tumor heterogeneity. Nat. Med. 21, 846-853 (2015).

3. Bachtiary, B. et al. Gene expression profiling in cervical cancer: an exploration of intratumor heterogeneity. Clin. Cancer Res. 12, 5632-5640 (2006).

4. Boyd, C. A., Benarroch-Gampel, J., Sheffield, K. M., Cooksley, C. D. \& Riall, T. S. 415 patients with adenosquamous carcinoma of the pancreas: a population-based analysis of prognosis and survival. J. Surg. Res. 174, 12-19 (2012).

5. Milosevic, M. F. et al. Interstitial fluid pressure in cervical carcinoma. Cancer 82, 2418-2426 (1998).

6. Lambin, P. et al. Decision support systems for personalized and participative radiation oncology. Adv. Drug Deliv. Rev. (2016). doi:10.1016/j.addr.2016.01.006

7. Steenbakkers, R., Duppen, J. C. \& Fitton, I. Observer variation in target volume delineation of lung cancer related to radiation oncologist-computer interaction: a 'Big Brother'evaluation. Radiother. Oncol. (2005).

8. Greco, C., Rosenzweig, K., Cascini, G. L. \& Tamburrini, O. Current status of PET/CT for tumour volume definition in radiotherapy treatment planning for non-small cell lung cancer (NSCLC). Lung Cancer 57, 125-134 (2007).

9. Sonke, J.-J. \& Belderbos, J. Adaptive radiotherapy for lung cancer. Semin. Radiat. Oncol. 20, 94-106 (2010).

10. van Baardwijk, A. et al. Individualized Radical Radiotherapy of Non-Small-Cell Lung Cancer Based on Normal Tissue Dose Constraints: A Feasibility Study. International Journal of Radiation Oncology*Biology*Physics 71, 1394-1401 (2008).

11. Zhou, J. et al. An automatic method for ground glass opacity nodule detection and segmentation from CT studies. Conf. Proc. IEEE Eng. Med. Biol. Soc. 1, 3062-3065 (2006).

12. Tao, Y. et al. Multi-level ground glass nodule detection and segmentation in CT lung images. Med. Image Comput. Comput. Assist. Interv. 12, 715-723 (2009).

13. Kubota, T., Jerebko, A. K., Dewan, M., Salganicoff, M. \& Krishnan, A. Segmentation of pulmonary nodules of various densities with morphological approaches and convexity models. Med. Image Anal. 15, 133154 (2011).

14. Lassen, B. C., Jacobs, C., Kuhnigk, J.-M., van Ginneken, B. \& van Rikxoort, E. M. Robust semi-automatic segmentation of pulmonary subsolid nodules in chest computed tomography scans. Phys. Med. Biol. 60, 1307-1323 (2015).

15. Tan, Y., Schwartz, L. H. \& Zhao, B. Segmentation of lung lesions on CT scans using watershed, active contours, and Markov random field. Med. Phys. 40, 043502 (2013).

16. Tachibana, R. \& Kido, S. Automatic segmentation of pulmonary nodules on CT images by use of $\mathrm{NCl}$ lung image database consortium. in Medical Imaging 61440M-61440M-9 (International Society for Optics and Photonics, 2006).

17. Buckler, A. J., Bresolin, L., Dunnick, N. R., Sullivan, D. C. \& Group. A collaborative enterprise for multistakeholder participation in the advancement of quantitative imaging. Radiology 258, 906-914 (2011).

18. Buckler, A. J. et al. Quantitative imaging test approval and biomarker qualification: interrelated but distinct activities. Radiology 259, 875-884 (2011).

19. Gatenby, R. A., Grove, O. \& Gillies, R. J. Quantitative imaging in cancer evolution and ecology. Radiology 269, 8-15 (2013).

20. Mohri, M., Rostamizadeh, A. \& Talwalkar, A. Foundations of Machine Learning. (MIT Press, 2012).

21. Pekalska, E. \& Duin, R. Foundations and Applications. (2005).

22. Guyon, I. \& Elisseeff, A. An Introduction to Variable and Feature Selection. J. Mach. Learn. Res. 3, 11571182 (2003).

23. Clauson, J., Hsieh, Y. C., Acharya, S. \& Rademaker, A. W. Results of the Lynn Sage Second-Opinion Program for local therapy in patients with breast carcinoma. Cancer (2002). 
24. Nguyen, P. L. et al. The impact of pathology review on treatment recommendations for patients with adenocarcinoma of the prostate. Urologic Oncology: Seminars and Original Investigations 22, 295-299 (2004/7).

25. Staradub, V. L., Messenger, K. A., Hao, N., Wiley, E. L. \& Morrow, M. Changes in breast cancer therapy because of pathology second opinions. Ann. Surg. Oncol. 9, 982-987 (2002).

26. Kronz, J. D., Westra, W. H. \& Epstein, J. I. Mandatory second opinion surgical pathology at a large referral hospital. Cancer 86, 2426-2435 (1999).

27. Bolón-Canedo, V., Sánchez-Maroño, N., Alonso-Betanzos, A., Benítez, J. M. \& Herrera, F. A review of microarray datasets and applied feature selection methods. Inf. Sci. 282, 111-135 (2014).

28. Brown, G., Pocock, A., Zhao, M.-J. \& Luján, M. Conditional Likelihood Maximisation: A Unifying Framework for Information Theoretic Feature Selection. J. Mach. Learn. Res. 13, 27-66 (2012).

29. Fernández-Delgado, M., Cernadas, E. \& Barro, S. Do we need hundreds of classifiers to solve real world classification problems. J. Mach. Learn. Res. (2014).

30. Oberije, C. et al. A prospective study comparing the predictions of doctors versus models for treatment outcome of lung cancer patients: a step toward individualized care and shared decision making. Radiother. Oncol. 112, 37-43 (2014).

31. Lambin, P., Roelofs, E., Reymen, B. \& Velazquez, E. R. Rapid Learning health care in oncology'-an approach towards decision support systems enabling customised radiotherapy. Radiother. Oncol. (2013).

32. Clark, K. et al. The Cancer Imaging Archive (TCIA): maintaining and operating a public information repository. J. Digit. Imaging 26, 1045-1057 (2013).

33. Clarke, L. P. et al. The Quantitative Imaging Network: NCl's Historical Perspective and Planned Goals. Transl. Oncol. 7, 1-4 (2014).

34. Gillies, R. J., Kinahan, P. E. \& Hricak, H. Radiomics: Images Are More than Pictures, They Are Data. Radiology 278, 563-577 (2016).

35. Haralick, R. M. Statistical and structural approaches to texture. Proc. IEEE 67, 786-804 (1979).

36. Gonzalez, R. C. \& Woods, R. E. Digital image processing. Nueva Jersey (2008).

37. Jain, A. K. Fundamentals of Digital Image Processing. (Prentice-Hall, Inc., 1989).

38. LeCun, Y., Bengio, Y. \& Hinton, G. Deep learning. Nature 521, 436-444 (2015).

39. Gao, M. et al. Segmentation label propagation using deep convolutional neural networks and dense conditional random field. IEEE ISBI (2016).

40. Liu, J. et al. COLITIS DETECTION ON COMPUTED TOMOGRAPHY USING REGIONAL CONVOLUTIONAL NEURAL NETWORKS. cs.jhu.edu

41. Shin, H.-C. et al. Deep Convolutional Neural Networks for Computer-Aided Detection: CNN Architectures, Dataset Characteristics and Transfer Learning. IEEE Trans. Med. Imaging 35, 1285-1298 (2016).

42. Jochems, A., Deist, T. M., van Soest, J. \& Eble, M. Distributed learning: predictive models based on data from multiple hospitals without data leaving the hospital. Radiother. Oncol. (2016). 



\section{Summary}

In order to enhance the applications of radiomics, in this thesis, we conducted different radiomic analyses on large patient cohorts of non-small cell lung cancer and head and neck cancer.

Part-1 Assessing variability in radiomics:

In the first part, the focus was on assessing publicly available semi-automatic segmentation tools. These tools were evaluated in terms of their efficiency and reproducibility and were compared to the manual segmentations. Also, reproducibility of radiomic features with respect to different segmentation methods were also assessed. Chapter- 2 \& 3 presented the studies where publicly available semiautomatic segmentation methods were assessed for their clinical relevance. Overall, it was observed that semiautomatic segmentation methods are tend to provide faster and more reproducible segmentations as compared to manual delineations. Moreover, in Chapter-4, It was observed that radiomic features extracted from the volumes, which are delineated by semiautomatic segmentation methods, had significantly higher reproducibility as compared to the features extracted from the manually segmented volumes. These results indicated that semiautomatic segmentation methods could provide a better alternative to the manual delineation for radiomic analyses.

\section{Part-2 Machine learning methods for radiomics}

In this part, different unsupervised and supervised machine learning methods were investigated for radiomics based prognostic/predictive analyses. These different methods of feature selection/reduction and prediction modeling were compared in terms of their performance and robustness. Chapter-5 presented a radiomic analysis of 1,019 patients with lung or head-and-neck cancer. It was found that radiomic signature have prognostic power in independent data sets of lung and head-and-neck cancer patients. Moreover, the radiogenomic analysis of this study revealed that a prognostic radiomic signature, capturing intratumor heterogeneity, was also associated with underlying gene-expression patterns. These independent cohorts were further investigated in Chapter- 6 for their cancer specific grouping and prognostic characteristics. This analysis indicated both common as well as cancer-specific clustering and clinical associations of 
radiomic features. In an exploratory study presented in Chapter-7, the association between radiomic features and the tumor histologic subtypes (adenocarcinoma and squamous cell carcinoma) were investigated in the cohorts of non-small cell lung cancer patients. Histological subtypes can influence the choice of a treatment/therapy for lung cancer patients. We observed that radiomic features show significant association with the lung tumor histology.

Highly accurate and reliable machine-learning approaches can drive the success of radiomic applications in clinical care. In Chapter-8 \& 9, different machine-learning methods were examined in terms of their performance and stability for predicting overall survival in independent lung and head \& neck cancer cohorts. To ensure the unbiased evaluation of different machine-learning methods, publicly available implementations along with reported parameter configurations were used. The variability analysis indicated that the choice of classification method was the most dominant source of performance variation. Identification of optimal machine-learning methods for radiomic applications is a crucial step towards stable and clinically relevant radiomic biomarkers, providing a non-invasive way of quantifying and monitoring tumor-phenotypic characteristics in clinical practice.

At the end, Chapter 10 provided a general discussion of the results presented in this thesis and related future perspectives. 


\section{Societal impact and valorizations}

In this thesis, state of the art computational approaches of mining non-invasive medical imaging data to derive predictive and prognostic inferences in clinical oncology are thoroughly described. Although the cohorts employed in this thesis were focused on specific oncological diseases - lung cancer and head and neck cancer, the described computational approaches harbor broad general applicability. In this section, we discuss the foreseeable societal impact of the presented research along with the policies that could be followed for the enhancement of personalized medicine.

We observe an exponential increase in data and information in this recent "big data" era. Healthcare is no different to this trend. The healthcare institutions of the developed countries archive immense amount of patient data and these practices are being adopted by the highly populated developing countries as well. This increasing amount of patient specific data could transform the routine patient care by tailoring and customizing treatments to individuals. More powerful predictive/prognostic tools are becoming available, enhancing the routine clinical care. This is aligned with the aimed personalized care - bringing the right treatment to the right patient. The use of predictive/prognostic models is increasing, with many online tools already available:

- http://www.predict.nhs.uk/ is an online tool used to help deciding the ideal course of treatment following breast cancer surgery, based on one's cancer histopathology;

- http://www.cancer.gov/bcrisktool/ allows estimating woman's risk of developing invasive breast cancer;

- https://www.mskcc.org/nomograms a long list of nomograms developed for a great variety of solid tumors, and based on hundreds or even thousands of patients, can be used to predict cancer outcomes or assess disease risk;

- http://www.predictcancer.org/ a platform making available published models developed at Maastro for lung, rectum, head and neck, and endometrium cancer

Furthermore, several collaborative consortiums for sharing data have been initiated:

- http://www.cancerimagingarchive.net/ is an online repository consisting imaging and clinical data related to different cancer types. It provides a platform to different healthcare institutions of the world to share data and collaborate research. 
- https://www.cancerdata.org/ is an initiative by Maastro clinic for sharing cancer data for research.

We believe that great knowledge gain could be obtained by data sharing. It allows having an access of external cohorts for validation, which is essential for the validity of research methodologies and proposed hypotheses of data driven research like Radiomics. Moreover, data sharing helps in reproducing the successful predictive/prognostic models, which latter could also be improved with additional data or by using better computational approaches.

As far as the research presented in this thesis is concerned, majority of the chapters employed one or more publicly available data. Furthermore, majority of the applied computational approaches and analysis tools are open source.

The first part of the thesis focused on evaluating different publicly available semiautomatic segmentation methods keeping in mind their applicability for raidomics. Two different semi-automatic segmentation approaches available in a free and publicly available 3D-Slicer software tool, were compared to the manual segmentations in terms of their efficiency and accuracy. 3D-Slicer is an open source software platform for medical image informatics, image processing, and three-dimensional visualization. Built over two decades through support from the National Institutes of Health and a worldwide developer community, Slicer brings free, powerful cross-platform processing tools to physicians, researchers, and the general public (https://www.slicer.org). Moreover, all the cohorts used in the first part of the thesis are available at TCIA (http://www.cancerimagingarchive.net) or at cancerdata.org.

The second part of the thesis covered the radiomics based predictive/prognostic analyses, where different machine learning approaches of feature selection and modelling were investigated for the radiomic cohorts of lung and/or head \& neck cancer patients. The work presented in chapter 5 , was one of the very first investigations investigating more than 400 quantitative imaging features in over a 1000 patients with lung and head and neck cancer, with independent validation datasets. We have shown for the first time the true independent validated impact of radiomics in both lung and head and neck cancer, showing the translational capability of our findings. Additionally, with the publication of the study presented in Chapter 5, we shared imaging datasets to the cancer imaging archive (TCIA) of 511 NSCLC patients of which 89 with gene-expression data. This again, will facilitate reproducibility of research and allow the investigation of other methodologies and hypotheses in the same data. This chapter received attention from the media, including a press release from the Maastricht University Medical Centre $^{1}$ and an internal press release in the Dana-Farber Cancer Institute in Boston, USA. This study was also featured in the outlook issue of the prestigious scientific magazine Nature, in a special issue on the outlook of lung cancer ${ }^{2}$.

The studies reported in chapters 6-9, were also one of those very first investigations investigating the impact of the advanced machine-learning approaches for radiomic analyses with large independent validation cohorts and two different cancer types. 
Predictive and prognostic models with high accuracy, reliability, and efficiency are vital factors driving the success of radiomics. Therefore, it is essential to compare different machine learning models for radiomics based clinical biomarkers. Moreover, we used open source analysis libraries and published parameter settings for the implementation of different machine learning methods enabling the unbiased evaluation of these methods. These articles have received good acceptance from the scientific community resulting in a decent number of citations within a short timespan.

Overall, in order to achieve significantly higher and positive societal impact, collaborative steps in terms of sharing data and knowledge are required. We are actively involved in these initiatives by participating in different data and tools sharing consortium and collaborating with different institutions across the glob. Our work has contributed towards the release of publicly available radiomics toolboxes ${ }^{3}$. By involving more institutions in sharing data and knowledge, we can evaluate the validity of radiomics based predictive/prognostic models, which could enhance the personalized medicine and hence the public health.

(1) http://maastrichtumchoofdsite.createsend1.com/t/ViewEmail/t/CADC497D2FEB9C5D

(2) http://www.nature.com/nature/journal/v513/n7517_supp/full/513S4a.html

(3) https://www.radiomics.io/code.html 



\section{Acknowledgements}

\section{THANK YOU!!!}

I dedicate this thesis to my parents Manjula and Mahesh Parmar. I am also grateful to my sisters and other family members for their love and support. I am thankful to my PhD advisors Prof. Philippe Lambin and Dr. Hugo Aerts for their help and support. I would also like to thank all of my mentors, teachers, colleagues and friends for their direct and indirect support to my doctoral research and life in general. Also, I was very fortunate to be associated with excellent academic institutions. Herewith, I express my gratitude to G. H. Patel College of Engineering and Technology, Dhirubhai Ambani Institute of Information and Communication Technology, Indian Statistical Institute, Maastro Clinic, Dana-Farber Cancer Institute, Brighma and Women's hospital, and Harvard Medical School. 



\section{Curriculum vitae}

Chintan Parmar was born on February 4'th, 1987 in Wankaner, Gujarat, India. He did his schooling from Wankaner, Surendranagar and Rajkot in Gujarat Secondary and Higher Secondary Educational Board. In 2004, he got enrolled into a four year bachelor of engineering (B.E.) degree program in computer engineering at G. H. Patel College of Engineering and Technology, Sardar Patel University, V. V. Nagar, Gujarat, India. During his under-graduation studies, he developed interest in computer science and artificial intelligence and decided to pursue further technical studies. In 2009, he qualified the graduate aptitude test in engineering (GATE) with a score of 94 percentile and got enrolled into a master of technology (M.Tech) program in Dhirubhai Ambani Institute of Information and Communication Technology (DAIICT).

At DAIICT, he studied advanced courses like pattern recognition, computer vision, information retrieval, image and signal processing etc. His master degree research was on automatic image inpainting, which is a computer vision and image analysis based approach for unobtrusively editing images. The research was focused on the images of vandalized monuments and the objective was to digitally reconstruct the vandalized regions of the monuments in images for the digital restoration of the ancient heritage sites. This research experience allowed him to gain good scientific aptitude and he decided to pursue a further research career by doing a doctoral studies. In 2011, he obtained his master of technology degree in information and communication technology with specialization in machine intelligence.

He started his PhD degree program with Maastro, Maastricht University, Netherlands in 2012. He also joined the Machine Intelligence Unit of Indian Statistical Institute, Kolkata as a research fellow in 2012 under the joint collaboration of Maastricht University and ISI-Kolkata. During his doctoral research, he also collaborated with researchers from Dana-Farber Cancer Institute and Harvard Medical School, Boston, USA.

He currently works as a research fellow at Dana-Farbar Cancer Institute, Brigham and Women's hospital and Harvard medical school, Boston, USA. 



\section{Scientific publications}

\section{Peer-reviewed Journal articles}

1. Wu, Weimiao*, Chintan Parmar*, Patrick Grossmann, John Quackenbush, Philippe Lambin, Johan Bussink, Raymond Mak, and Hugo J. W. L. Aerts. 2016. "Exploratory Study to Identify Radiomics Classifiers for Lung Cancer Histology." Frontiers in Oncology 6 (March). ncbi.nlm.nih.gov: 71.

2. Parmar, Chintan*, Patrick Grossmann*, Derek Rietveld, Michelle M. Rietbergen, Philippe Lambin, and Hugo J. W. L. Aerts. 2015. "Radiomic Machine-Learning Classifiers for Prognostic Biomarkers of Head and Neck Cancer." Frontiers in Oncology 5 (December). ncbi.nlm.nih.gov: 272.

3. Parmar, Chintan*, Patrick Grossmann*, Johan Bussink, Philippe Lambin, and Hugo J. W. L. Aerts. 2015. "Machine Learning Methods for Quantitative Radiomic Biomarkers." Scientific Reports 5 (August). nature.com: 13087.

4. Parmar, Chintan*, Ralph T. H. Leijenaar*, Patrick Grossmann, Emmanuel Rios Velazquez, Johan Bussink, Derek Rietveld, Michelle M. Rietbergen, Benjamin Haibe-Kains, Philippe Lambin, and Hugo J. W. L. Aerts. 2015. "Radiomic Feature Clusters and Prognostic Signatures Specific for Lung and Head \& Neck Cancer." Scientific Reports 5 (June). nature.com: 11044.

5. Parmar, Chintan*, Emmanuel Rios Velazquez*, Ralph Leijenaar, Mohammed Jermoumi, Sara Carvalho, Raymond H. Mak, Sushmita Mitra, et al. 2014. "Robust Radiomics Feature Quantification Using Semiautomatic Volumetric Segmentation." PloS One 9 (7). journals.plos.org: e102107.

6. Aerts, Hugo J. W. L.*, Emmanuel Rios Velazquez*, Ralph T. H. Leijenaar, Chintan Parmar, Patrick Grossmann, Sara Cavalho, et al. 2014. "Decoding Tumour Phenotype by Noninvasive Imaging Using a Quantitative Radiomics Approach." Nature Communications 5 (June). nature.com: 4006.

7. Velazquez, Emmanuel Rios*, Chintan Parmar*, Mohammed Jermoumi, Raymond H. Mak, Angela van Baardwijk, Fiona M. Fennessy, John H. Lewis, et al. 2013. "Volumetric CT-Based Segmentation of NSCLC Using 3D-Slicer." Scientific Reports 3 (December). nature.com: 3529.

8. Carvalho, Sara*, Ralph T. H. Leijenaar*, Emmanuel Rios Velazquez, Cary Oberije, Chintan Parmar, Wouter van Elmpt, Bart Reymen, et al. 2013. "Prognostic Value of Metabolic Metrics Extracted from Baseline Positron Emission Tomography Images in Non-Small Cell Lung Cancer." Acta Oncologica 52 (7). Taylor \& Francis: 1398-1404. 
9. Leijenaar, Ralph T. H., Sara Carvalho, Emmanuel Rios Velazquez, Wouter J. C. van Elmpt, Chintan Parmar, Otto S. Hoekstra, Corneline J. Hoekstra, et al. 2013. "Stability of FDG-PET Radiomics Features: An Integrated Analysis of Test-Retest and Inter-Observer Variability." Acta Oncologica 52 (7). Taylor \& Francis: 1391-97.

10. Stephen Yip*, Jhon Kim*, Thibaud P. Coroller, Chintan Parmar, et al. 2017. "Associations between somatic mutations and metabolic imaging phenotypes in non-small cell lung cancer." Journal of nuclear medicine 58 (4). Society of Nuclear Medicine: 569-576.

11. Elizabeth Huynh, Thibaud P Coroller, Vivek Narayan, Vishesh Agrawal, John Romano, Idalid Franco, Chintan Parmar, et al. 2017. "Associations of Radiomic Data Extracted from Static and Respiratory-Gated CT Scans with Disease Recurrence in Lung Cancer Patients Treated with SBRT." PlosOne 12 (1). Public Library of Science.

\section{CONFERENCE PROCEDDINGS}

1. Milind G Padalkar, Manjunath V Joshi, Mukesh A Zaveri, and Chintan Parmar. 2012. Exemplar based inpainting using autoregressive parameter estimation. In Proceedings of the International Conference on Signal, Image and Video Processing, ICSIVP, 12 (1). 154-160.

2. Chintan Parmar, Manjunath V Joshi, Mehul Raval, Mukesh A Zaveri. 2011. Automatic image inpainting for the facial images of monuments. In Proceedings of the Electrical Engineering Centenary Conference. 415-420.

\section{ABSTRACTS}

1. Yip, S., J. Kim, T. Coroller, E. R. Velazquez, and C. Parmar. 2016. "MO-DE-207B-01: JACK FOWLER JUNIOR INVESTIGATOR COMPETITION WINNER: Between Somatic Mutations and PET-Based Radiomic Features in Non-Small Cell Lung Cancer. " Medical Physics 43(6). American Association of Physicists in medicine. 3704-3704

2. Velazquez, E. R., Y. Liu, C. Parmar, and V. Narayan. 2016. "MO-DE-207B-08: Radiomic CT Features Complement Semantic Annotations to Predict EGFR Mutations in Lung Adenocarcinomas." Medical Physics 43(6). American Association of Physicists in medicine. 3706-3706.

3. Grossmann, P., O. Grove, N. El-Hachem, E. Rios Velazquez, C. Parmar, R. Leijenaar, B. HaibeKains, P. Lambin, R. Gillies, and H. Aerts. 2015. "TU-CD-BRB-02: BEST IN PHYSICS (JOINT IMAGING-THERAPY): Identification of Molecular Phenotypes by Integrating Radiomics and Genomics." Medical Physics 42 (6). American Association of Physicists in Medicine: 3602-3602.

4. Velazquez, E. R., C. Parmar, M. Jermoumi, and H. Aerts. 2014. "TU-A-12A-10: Robust Radiomics Feature Quantification Using Semiautomatic Volumetric Segmentation." Medical Physics 41(6). American Association of Physicists in medicine. 452-452. 


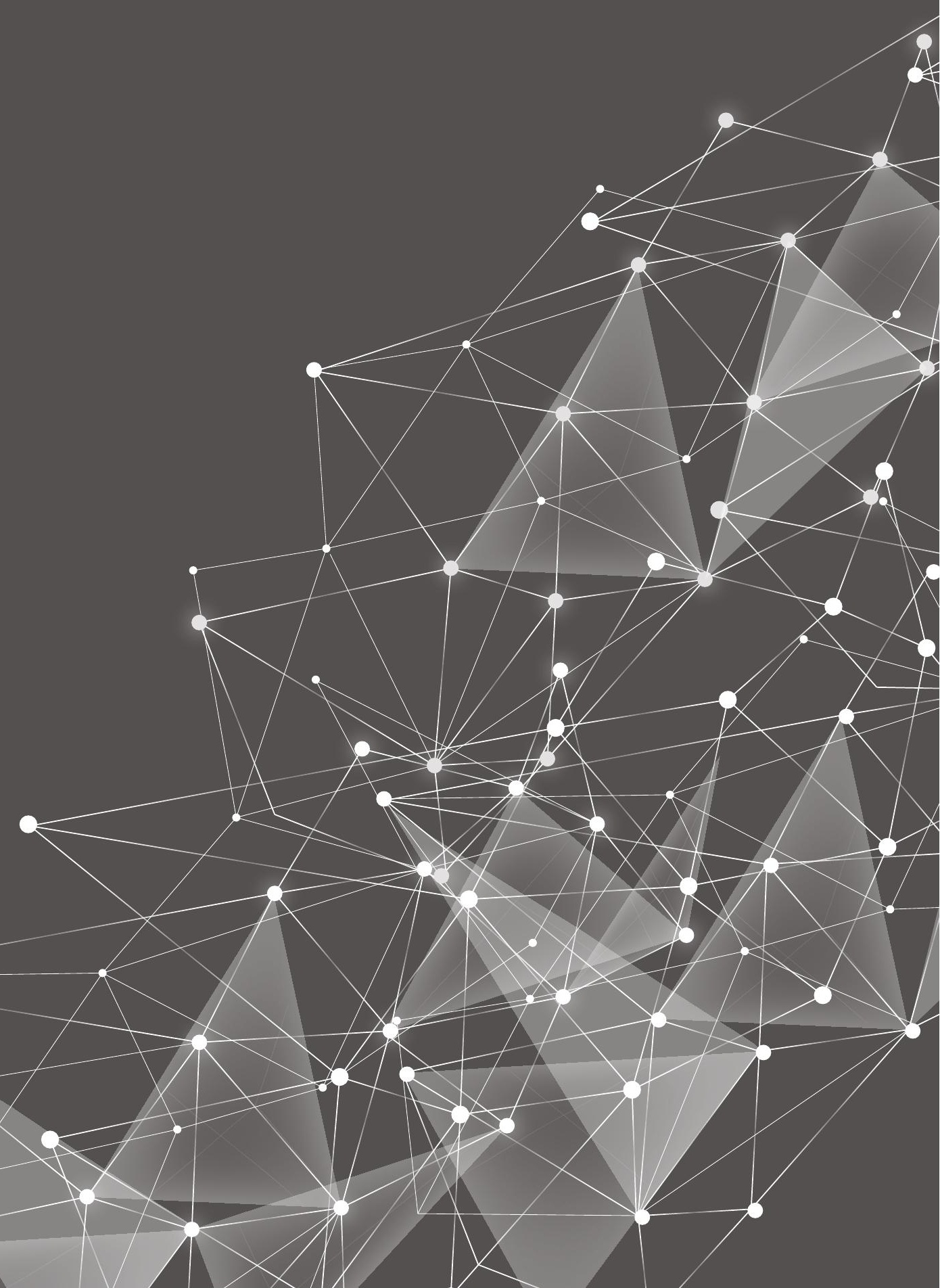

